\title{
An Investigation of Fatigue in Stationary Biking with Application to Iliotibial Band Syndrome Development
}

Submitted to the Department of Mechanical and Aerospace Engineering

at the

UNIVERSITY OF VIRGINIA

Charlottesville, Virginia, USA

In partial fulfillment of the requirements for the degree of Master of Science in Mechanical and Aerospace Engineering

By

Deepak Sathyanarayan

B.S. Biomedical Engineering, Duke University, 2015

December 2017 


\section{Committee Members}

Committee Chair

Committee Member

Committee Member

Primary Advisor

\section{Jason Kerrigan, Ph.D.}

Assistant Professor

Center for Applied Biomechanics

Department of Mechanical and Aerospace Engineering University of Virginia

Joe M. Hart, Ph.D., A.T.C.

Assistant Professor

Department of Orthopedic Surgery

University of Virginia

\section{Gregory J. Gerling, Ph.D.}

Associate Professor

Department of Systems \& Information Engineering

University of Virginia

\section{Matthew B. Panzer, Ph.D.}

Assistant Professor

Center for Applied Biomechanics

Department of Mechanical and Aerospace Engineering University of Virginia 


\title{
An Investigation of Fatigue in Stationary Biking with Application to Iliotibial Band Syndrome Development \\ by \\ Deepak Sathyanarayan
}

\begin{abstract}
Submitted to the Department of Mechanical and Aerospace Engineering on December 11, 2017, in partial fulfillment of the requirements for the degree of Master of Science in Mechanical and Aerospace Engineering
\end{abstract}

\begin{abstract}
Cycling, or spinning (stationary biking), is a sport that has a low incidence of acute injury and is considered a safe, low impact, cardio workout. However, the repetitive behavior of cycling makes the rider especially prone to the development of long-term injury. In literature, the cause of these injuries has been theorized to have multiple contributors, such as improper riding technique, poor compensation strategies, and overall muscle fatigue. The primary aim of this thesis is to advance the current understanding of human performance and fatigue processes by combining kinematic, kinetic, and muscle activation data from an experimental stationary bike test to identify performance metric trends, analyze EMG-based regression models of performance loss, and develop subject-specific musculoskeletal models to estimate iliotibial band syndrome (ITBS) risk factors, lateral femoral epicondyle (LFE) compression force and impingement duration. Highdimensional data was collected from sEMG-fitted volunteers as they were guided through a 1-hour stationary biking endurance routine. Motion capture and novel bike instrumentation were utilized to collect detailed kinematic and kinetic data with minimal influence on cycling performance. Following a warm-up period, pedaling resistance was manually incremented to maintain a selfreported high resistance level and reflect group stationary biking training. Volunteer fatigue levels were categorized by the extent of cadence reduction under constant cycling difficulty. Fatigued and non-fatigued performance groups exhibited significant differences, with the Fatigued group showing: greater peak hip adduction, greater lumbar flexion, greater torso and pelvic center-ofmass motion, and greater reductions in muscle activation amplitude. EMG signal shape parameters from the fatigue group were used to develop linear regression models of performance reduction. Correlation analysis and model fitting revealed a strong significance of distal motor control loss (foot plantar-/dorsi-flexion muscles), not power generation muscles, in triggering system-level performance change. OpenSim muscle path biofidelity was improved to match literature cadaveric muscle data, and estimation of ITBS risk factors was implemented. Model predictions indicate fatigued individuals have increased LFE compression duration, but decreased compression force due to kinematic changes. While osteokinematic and muscle activation compensation mechanisms were observed to maintain cadence among the non-fatigue group, increased LFE compression force was predicted with continued cycling. The investigation highlights the degradation of technique and elevated injury risk associated with fatigue and compensation processes, respectively. The identified performance-critical muscle parameters and fatigue-induced kinematic changes that can be used to inform overuse injury prevention in clinical rehabilitation, athletic training, and military applications.
\end{abstract}

Thesis Supervisor: Matthew B. Panzer

Center for Applied Biomechanics, University of Virginia 


\section{Acknowledgements}

\section{Thank you to the many mentors and researchers who supported the presented work - without whom, the opportunity to grow as a scientist, and as a person, would not be so fruitful.}

At every stage of my time at the University of Virginia, whether at the CAB, on Grounds, or out on the Downtown Mall, kind-hearted and supportive people were there to help me over the hurdles and celebrate the successes. My fellow CAB graduate students were daily motivators and sounding boards for research and life, and are the connective tissue of what made the CAB such a close and functional community. I will always cherish the dinners we shared and those many trials with pupusas. Though CAB is away from the bustle of Grounds, I was fortunate to make a number of close undergraduate and graduate friends across UVA. From Hyperloop competitions, to cultural performances, to the 72-hour sprint to propose the Tinker Labs Initiative, the comradery we grew will be aspired for, but likely never replicated.

What is now my master's thesis was initially started as a pilot study with support from the Double Hoo Research Grant. Continued support from Brian Cullaty and the Center for Undergraduate Excellence enabled us to not only successfully complete the study, but also share the results at several conferences over these two years. Working long nights at CAB and as we traveled across the U.S., undergraduate Austin Rivera will always be an invaluable collaborator in and out of the lab setting. Of equal importance, UVA research staff were a wealth of technical and professional expertise. A special thanks to Ali Forghani for passing on his knowledge of motion capture so that the research could continue independently in resource-tight times at CAB. Thank you to Ted Miller and Tim Gillespie for nimble coordination of internal CAB logistics and resources. It was a sight to see the beautiful synergy between machining from Brian Overby and Joey White, and sensor know-how from Kevin Kopp that brought my instrumentation designs to life. IRB approval was made possible with the IT and clinical support of Mark McCardell, Thomas Hartka, and Susie Hoffman. I am also grateful to all the C-Ville cycling volunteers who enthusiastically offered their afternoons to science.

For the invaluable conceptual foundation and constructive feedback that allowed me to grow academically, I am deeply appreciative of the faculty and mentors I have worked with over the years at UVA. Thank you to my thesis committee members and my advisor for the support and advice that allowed me to successfully complete my contribution to this investigation.

Last, but certainly not least, the emotional Atlas that is my family and relatives. While hundreds of miles away, your love was felt wherever I was at UVA. Thank you for always being by my side through my many ups and downs over the years. In the spirit of pushing through the journey ahead, unifying words after a particularly memorable work night, "It was worth it". 


\section{Table of Contents}

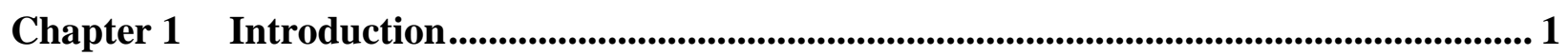

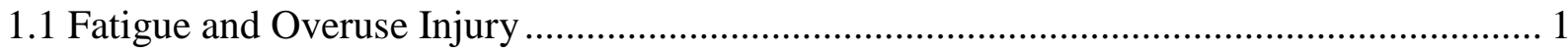

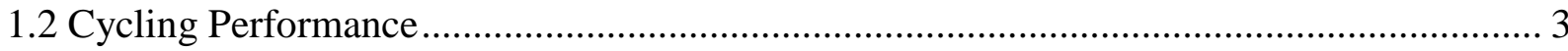

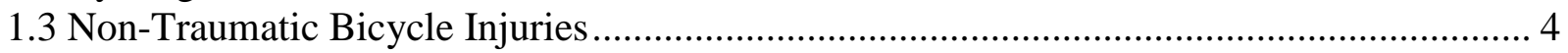

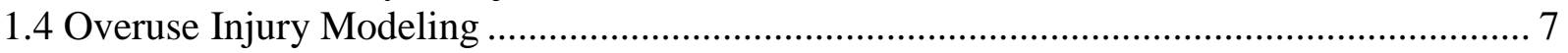

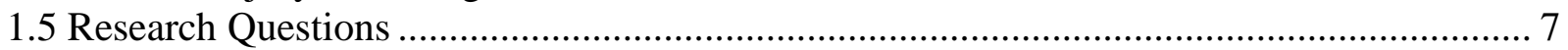

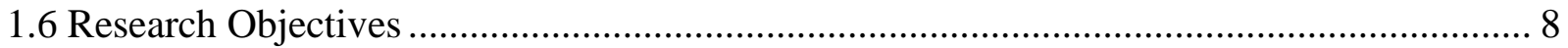

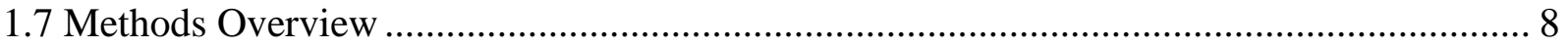

Chapter 2 Performance Description................................................................................................... 10

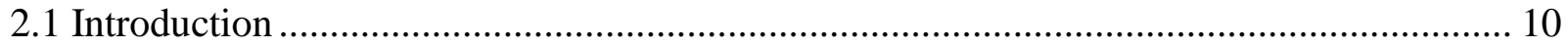

2.2 Chapter Objectives ……………………………............................................... 10

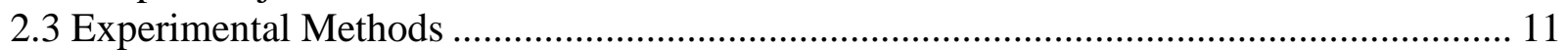

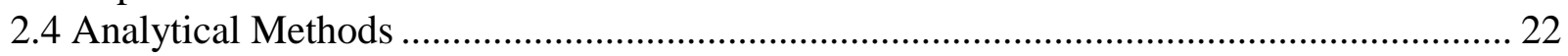

2.5 Volunteer Testing Summary ………………………................................................ 29

2.6 Performance Results................................................................................................. 31

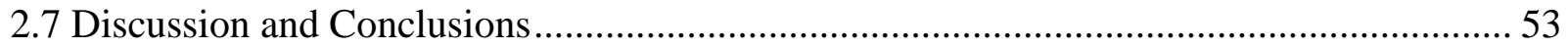

Chapter 3 EMG-Based Regression Analysis ...............................................................................5 59

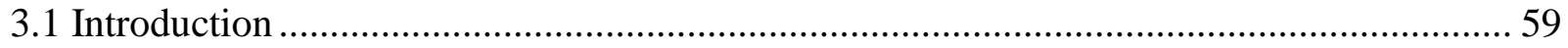

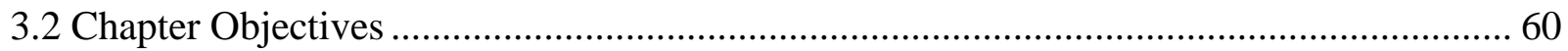

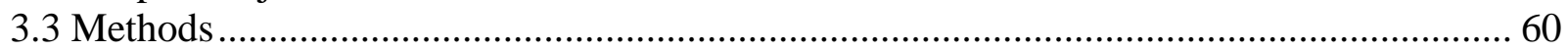

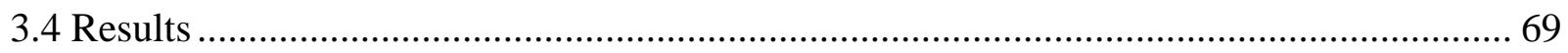

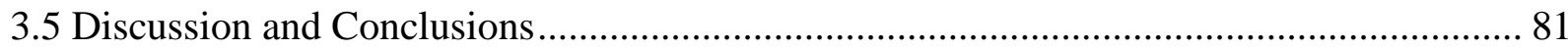

Chapter 4 IT-Band OpenSim Model.................................................................................. 86

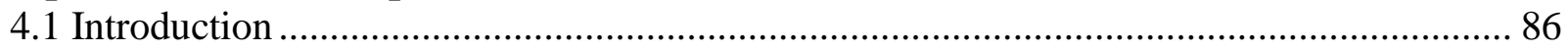

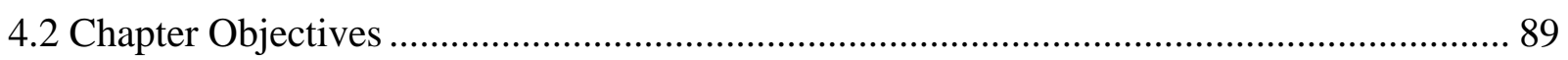

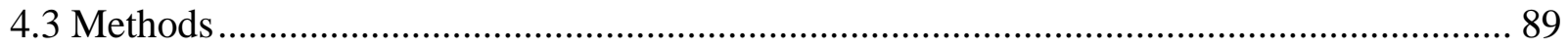

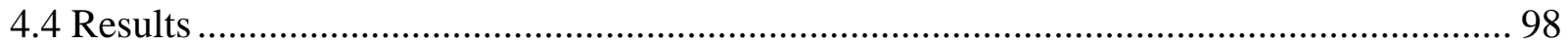

4.5 Discussion and Conclusions .................................................................................... 110

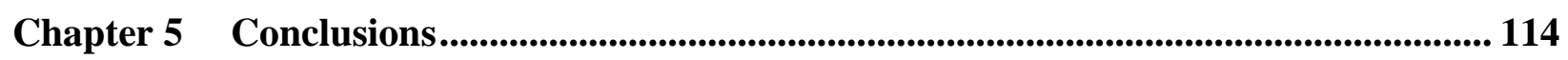

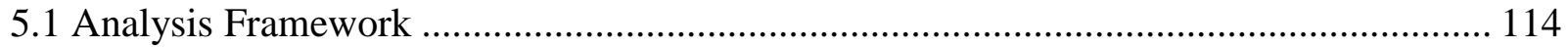

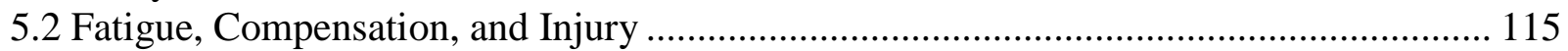

5.3 EMG-Based Performance Models ……………….................................................... 116

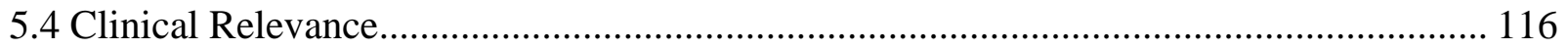


Chapter 6 Limitations \& Extensions ........................................................................... 120

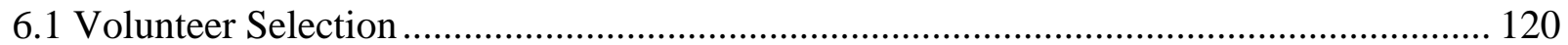

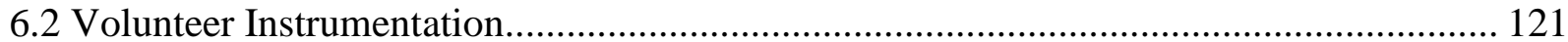

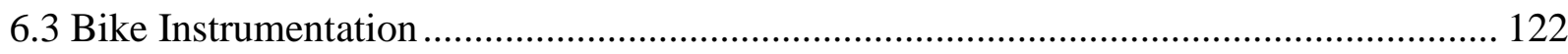

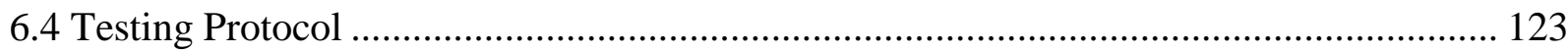

6.5 Follow-Up Survey \& Longitudinal Tracking ....................................................... 124

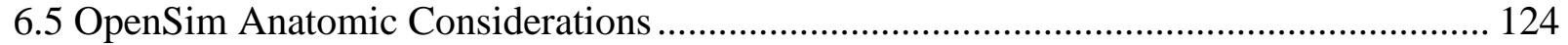

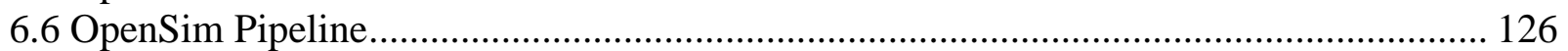

6.8 Performance Model Generalization ........................................................................ 127

6.9 Finite Element Knee Model ................................................................................. 127

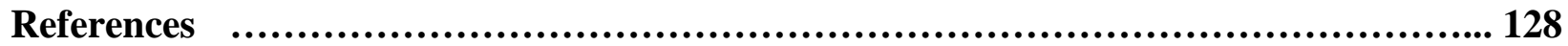

Appendix A Volunteer Characteristics ................................................................................. 135

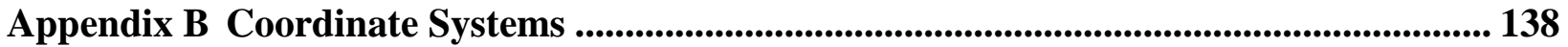

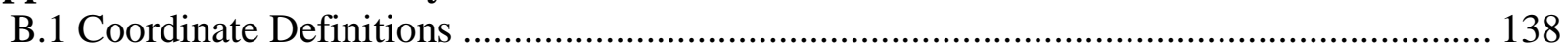

B.2 Coordinate Transformations ........................................................................... 140

Appendix C IRB Forms Consent \& Recruitment .................................................................. 142

Appendix D Engineering Drawings ................................................................................... 143 


\section{List of Figures}

\section{Chapter 1 Figures}

Figure 1-1: Hypothetical Model of Overuse Injury (from Clarsen 2015) .................................... 1

Figure 1-2: Current Overuse Injury Development Schema .................................................... 2

Figure 1-3: ITBS Injury Site [modified from Baker et al.].................................................... 5

Figure 1-4: Conducted Analytical Framework for Real-Time Human Performance Metrics....... 8

\section{Chapter 2 Figures}

Figure 2-1: Bike Instrumentation Diagram. Blue arrows indicate bike adjustment joint motions.

Figure 2-2: Tioga "Zero-Axel" Spindle-less Pedal Bearing with Tioga Pedal.............................. 14

Figure 2-3: Crankarm-Mounted Instrumented Pedal without Foot Plate Harness Attached ........ 14

Figure 2-4: Frame-Mounted Instrumented Seat Post without Seat Cushion Attached ................. 15

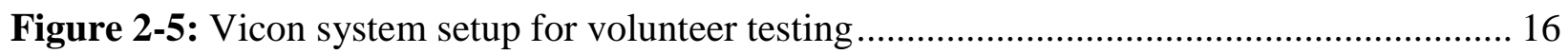

Figure 2-6: Surface EMG Sensor Placement for Volunteer Tests ............................................. 17

Figure 2-7: Vicon Marker Placement for Volunteer Tests ...................................................... 19

Figure 2-8: Vicon Marker Placements on Volunteer and Bike Segments .................................. 20

Figure 2-9: Profile of self-selected resistance level over endurance test...................................... 21

Figure 2-10: Right Pedal Coordinate System and Vicon Marker Cluster .................................. 22

Figure 2-11: Right Pedal, Crank, and Global Coordinate Systems and Transformations ........... 24

Figure 2-12: Displacement Vector Definition for Power Calculations ..................................... 25

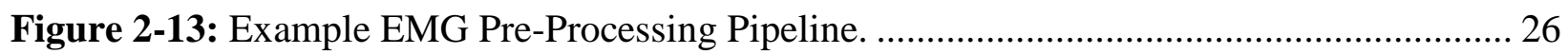

Figure 2-14: Figure Format Used for Presentation of Parameter Trends .................................... 28

Figure 2-15: RPM Profiles Observed in Endurance Ride........................................................ 32

Figure 2-16: Mechanical Power and Force Application Efficacy for Fatigue and Non-Fatigue

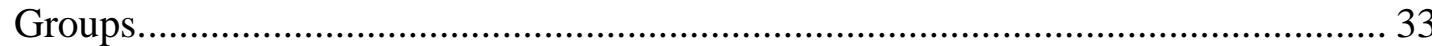

Figure 2-17: Change in Force Application Efficacy for Fatigue and Non-Fatigue Groups. ........ 34

Figure 2-18: Average EMG Profiles for Distal Leg Muscle Groups............................................ 35

Figure 2-19: Average EMG Profiles for Proximal Leg Muscle Groups....................................... 37

Figure 2-20: Polar Representation of EMG Activation Profiles................................................. 39

Figure 2-21: Pedal Orientation and Ankle Dorsiflexion Over Cycle During Select Regions of Endurance Ride.

Figure 2-22: Knee Extension Over Cycle During Iso-effort Region of Endurance Ride for Fatigue and Non-Fatigue Groups.

Figure 2-23: Raw and Average Hip Kinematic Angles Over Cycle in Pre-Fatigue and Fatigue States.

Figure 2-24: Pelvis COM Translation Over Cycle During Endurance Ride. 
Figure 2-25: Torso COM Translation Over Cycle During Endurance Ride. 48

Figure 2-26: Coronal-view of Average Torso COM and Pelvis COM Trajectories Over Cycle

During Isokinetic Region of Endurance Ride for Fatigue and Non-Fatigue Groups. 49

Figure 2-27: Lumbar Extension Over Cycle During Iso-effort Region of Endurance Ride for

Fatigue and Non-Fatigue Groups 50

\section{Chapter 3 Figures}

Figure 3-1: Muscles of interest for EMG Placement and Measurement ................................ 61

Figure 3-2: Schematic of example EMG signal thresholding and parameters measured........... 63

Figure 3-3: EMG-based Model Development Pipeline ..................................................... 65

Figure 3-4: Normalized RPM Profiles for Reevaluation of "Fatigue" and "Non-Fatigue"

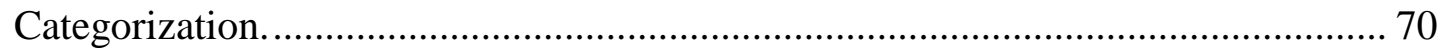

Figure 3-5: Univariate Correlation Coefficients and RPM Predictor Selection....................... 71

Figure 3-6: Covariance Matrices for Initial Set of Model Parameters. ................................... 72

Figure 3-7: Change in Adjusted R2-value with Robust Stepwise Backward Elimination

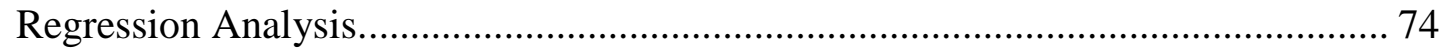

Figure 3-8: Fatigue Group Model Predictions and Residual Probability Density Plots............. 77

Figure 3-9: Non-Fatigue Group Model Predictions and Residual Probability Density Plots...... 78

Figure 3-10: Conversion from PRESS to Average Prediction Error for N=500 Observations ... 79

Figure 3-11: Prediction $\mathrm{R}^{2}$-values and Predicted Response from PRESS Calculations............. 80

\section{Chapter 4 Figures}

Figure 4-1: Anatomical Considerations for EMG Placement and OpenSim Model Development

Figure 4-2: Lumped-Parameter Muscle Model from Thelen 2003....................................... 88

Figure 4-3: Final marker placement for OpenSim model ................................................. 90

Figure 4-4: Overview of OpenSim Model Development Process ......................................... 91

Figure 4-5: Moment arm corridors from Eng et al. (2015) used for improving biofidelity of OpenSim model predictions of TFL forces ....................................................... 92

Figure 4-6: Implementation of wrap surfaces for TFL moment arm correction. ..................... 93

Figure 4-7: MRI images from Flato et al. (2017) of symptomatic knees from ITBS patients. ... 94

Figure 4-8: Models considered for LFE contact surface definition...................................... 94

Figure 4-9: OpenSim Analysis Pipeline. ....................................................................... 95

Figure 4-10: TFL Moment Arm Corridor Fitting. …...................................................... 99

Figure 4-11: Static Pose Dynamic Activation Simulation to Evaluate TFL Muscle Model. .... 100

Figure 4-12: TFL Force Response to Single DOF Hip and Knee Kinematics under Constant

Activation (100\%) and Constant Fiber Length (7mm) .................................... 101

Figure 4-13: Normalized 2-DOF TFL Force Response Surface.......................................... 102

Figure 4-14: LFE Model Force Predictions...................................................................... 103

Figure 4-15: Developed OpenSim Model with TFL Wrap Surfaces ..................................... 104 
Figure 4-16a: Normalized Fatigue TFL Force Response Surface with Average Kinematic Overlays.

Figure 4-16b: Normalized Non-Fatigue TFL Force Response Surface with Average Kinematic

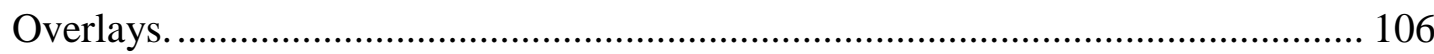

Figure 4-17a: Normalized Fatigue TFL force versus crank angle.................................... 107

Figure 4-17b: Normalized Non-Fatigue TFL force versus crank angle. ............................. 107

Figure 4-18a: Predicted Fatigue LFE compression force versus crank angle....................... 108

Figure 4-18b: Predicted Non-Fatigue LFE compression force versus crank angle................. 109

Figure 4-19: Impingement Zone Range-of-Motion Changes for Non-Fatigue and Fatigue

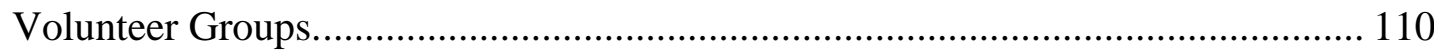

\section{Chapter 5 Figures}

Figure 5-1: Proposed Analytical Framework for Real-Time Human Performance Metrics

\section{Chapter 6 Figures}

Figure 6-1: Distal Femoral Attachments of the ITB

\section{Appendix Figures}

Figure B-1: Loadcell Local Coordinate System Definitions for Pedals and Seat 138

Figure B-2: Pedal, Global, and Crank Arm Coordinate System Definition for the Right Pedal 139

Figure B-3: Pedal, Global, and Crank Arm Coordinate System Definition for the Left Pedal . 139

Figure B-4: Seat and Global Coordinate System Definition for the Seat 


\section{List of Tables}

\section{Chapter 1 Tables}

Table 1-1: Parameters Collected from Endurance Ride

\section{Chapter 2 Tables}

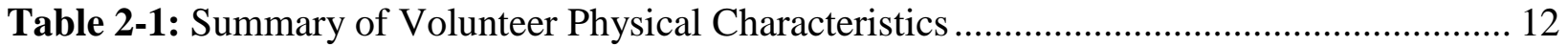

Table 2-2: Summary of Self-Reported Competition and Training History ………..................... 12

Table 2-3: Summary of Test Resistance Adjustments and Comments ......................................... 29

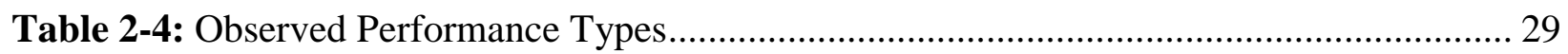

Table 2-5: Volunteer RPM Changes and Performance Types over Trial .................................. 30

Table 2-6: Summary of Volunteer Physical Characteristics by Performance Category ............... 31

Table 2-7: Isokinetic Regions from "Non-Fatigue" Volunteers Used for Performance Analysis 32

Table 2-8: Student's t-Test Results for Volunteer Parameters versus Performance Category .... 51

\section{Chapter 3 Tables}

Table 3-1: Models used to Evaluate Non-Fatigue Performance Prediction........................................ 69

Table 3-2: Initial Parameters Down-Selected from Covariant Removal ....................................... 73

Table 3-3: Final Model Parameters from Stepwise Backward Elimination Regression Analysis 74

Table 3-4: Group Model Coefficients Ordered by Parameter Significance................................. 75

Table 3-5: Summary of RMSE from Group Model Predictions of Subject Data ........................ 76

Table 3-6: Summary of PRESS from Subject-Specific Fitting of Group Models ........................ 79

Table 3-7: Summary of Prediction $R^{2}$ from Subject-Specific Fitting of Group Models .............. 81

\section{Chapter 4 Tables}

Table 4-1: Simulations Run for Evaluation of Updated TFL Model

\section{Appendix Tables}

Table A-1: Summary of Volunteer Physical Characteristics ................................................ 135

Table A-2: Summary of Self-Reported Prior Injuries......................................................... 135

Table A-3: Summary of Self-Reported Competition and Training History ............................. 136

Table A-4: Summary of Test Setup Characteristics.............................................................. 136

Table A-5: Summary of Volunteer Physical Characteristics by Performance Category ........... 137 


\section{Chapter 1}

\section{Introduction}

\subsection{FATIGUE AND OVERUSE INJURY}

As engagement in personal fitness and team sports increases in the general population (The Physical Activity Council 2017; National Council of Youth Sports 2008), a focus on safe technique is crucial to slow the increase of training-related overuse injuries (Öztürk 2013). Overuse injuries, such as tendinopathy, can have significant effects on society by reducing worker performance and limiting the success of athletes and other physically demanding professions (Clarsen 2015). However, unlike acute injury, overuse injury development are not easily tracked by clinical visits or monetary impact until significantly limiting performance (Hopkins et al. 2016). Figure 1-1 visually represents a potential explanation for under-representation of overuse injury incidence and economic impact in literature (Clarsen 2015).

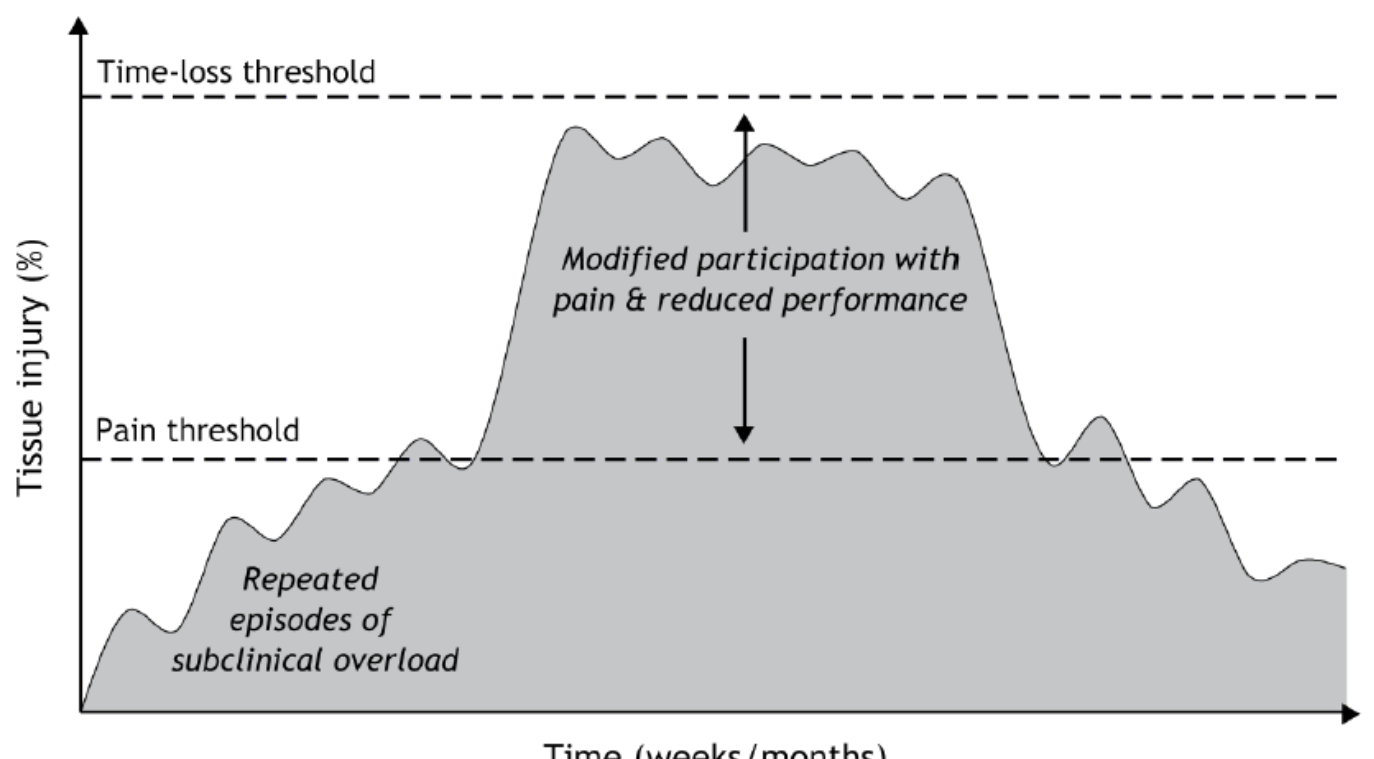

Time (weeks/months)

Figure 1-1: Hypothetical Model of Overuse Injury (from Clarsen 2015). 
Overuse injuries also have severe health implications after long-term repeated exposure to a stressor (Sperryn 1994; de Bernardo et al. 2012; Petchprapa and Bencardino 2013). Generally preceded with benign symptoms such as light pain, overuse injury can initiate a cascade of additional joint and tissue damage, even resulting in acute musculoskeletal failure if left untreated (Sperryn 1994). Early detection and treatment of the early stages of these injury mechanisms are essential in preventing the catastrophic issues in the future.

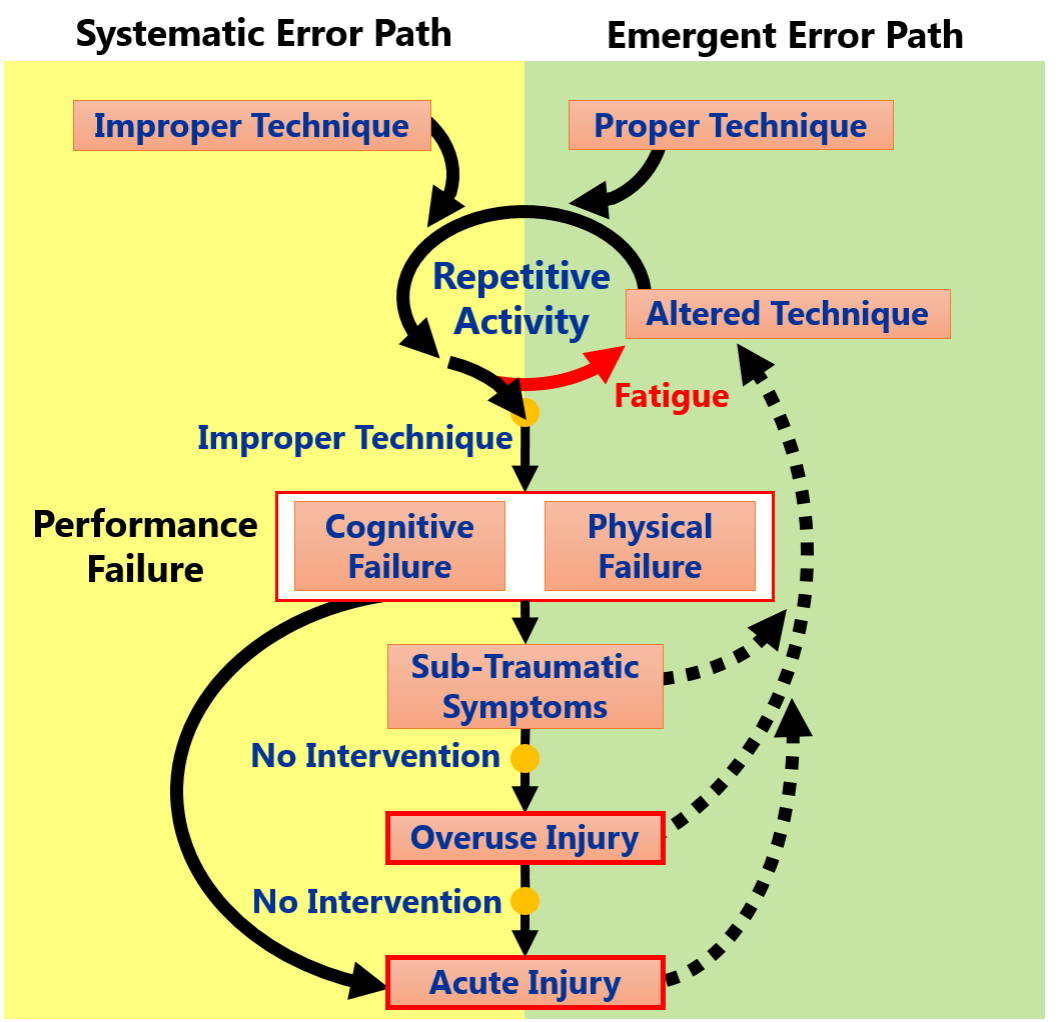

Figure 1-2: Current Overuse Injury Development Schema

The development of overuse injury can be attributed to either systematic or emergent performance errors [Figure 1-2]. While systematic errors can be readily detected and corrected in moderate intensity training, emergent performance errors develop during an activity (Callaghan 2005). Human error can emerge through a variety of pathways, generally grouped into cognitive and physical performance failure (Noakes 2000). Extensive research is done on both failure mechanisms, and depending on the field of application, the term "fatigue" can pertain exclusively to one or both types of performance (Enoka and Duchateau 2016). For the purposes of this study, an individual's "fatigue" in an activity will encompass physical (i.e. kinematic, kinetic, and physiological) forms of task-oriented performance loss. The emergence and effects of fatigue in 
an activity can vary significantly on an individual basis. It is the goal of human performance research to develop the knowledge necessary to safely account for that variability in human-factors and biomedical design.

In the field of medicine, physiological fatigue and endurance has application in assessing the health of multiple organ systems as we age, including pulmonary, cardiovascular, and musculoskeletal performance. Physiological endurance on the $400 \mathrm{~m}$ walk has been shown to be a strong predictor of mortality of older adults (Vestergaard et al. 2009). Fatigue development patterns also offer diagnostic value for inflammatory diseases, like multiple sclerosis (MS), where the development of MS leads to impeded voluntary motion of the upper extremities and increased fatigability (Kos et al. 2008). In high performance environments like military and active sports, the effects of

performance fatigue have been seen to lead to musculoskeletal injury and fracture. Lower extremity pathophysiology of stress fracture often emerges from the decline of muscular support, leading to reduced support of underlying bone, and subsequent excessive off-nominal load transmission (Springer and Ross 2013). Runners and running-intensive athletes are often afflicted by hip and knee overuse injuries, where injury symptoms do not typically manifest as fracture, but rather as painful inflammation of the joint tissues after even short durations of training (Brown et al. 2016). Among the active population, overuse injury is prevalent across age, gender, and size demographics (Enoka and Duchateau 2016; Jayanthi et al. 2013).

While fatigue is critical to all areas of medicine, military, and sports, the following investigation aims to develop a general understanding of physiology fatigue processes through the context of the high repetition and long duration sport of cycling.

\subsection{CYCLING PERFORMANCE}

Although one of the lowest impact sports, cycling places a high demand on athlete cardiovascular and musculoskeletal performance. Like any human-machine system, the fitting of the bike to the rider anthropometry plays an important role in overall system efficacy. Adversely, poor compatibility can reduce cycling power generation and lead to long-term injury development (Gregor, Broker, and Ryan 1991). Given proper initial bike setup, the main objectives for the 
athlete to perform effectively pertain to force generation, direction of application, and timing. Accomplishing this emerges through synergy between body kinematics and muscle activity. Multiple combinations of kinematic-kinetic patterns for power generation exist, and the human body responds differently to each (Lanferdini et al. 2016). Altered joint kinematics in cyclic activities are a primary trait of increasing injury development risk, and the onset of this change can vary based on physical fitness and training technique. During short time trials, competitive cyclists do not exhibit significant sagittal-plane kinematic differences from recreational cyclists (Rodrigo R. Bini et al. 2016). However, during long duration trials, when fatigue and endurance effects are magnified, recreational participants exhibit increased torso lateral motion and flexion relative to competitive cyclists (Van Hoof et al. 2012). Upper extremity and torso stability is also a focus of cycling performance. While lower extremity kinematics drive muscular power generation, the inertial effects of torso and pelvis motion can supplement force transmission to the pedals or accelerate fatigue onset (Abt et al. 2007). Not only does core instability have implications in fatigue, but due to the kinematic-constraints on the pelvis (seat) and feet (pedals), slight variations in pelvis tilt can lead to excessive loading of the knee and ankle (Abt et al. 2007; Bailey, Maillardet, and Messenger 2003). Through muscle weakness or technique, asymmetry in osteokinematics and joint loading has also been shown to correlate with fatigue onset and subsequent injury development mechanisms (Rodrigo Rico Bini and Hume 2012; Bailey, Maillardet, and Messenger 2003). While the injury outcome and hypothesized cause are correlated, there is scarce literature on the internal joint loading changes in long-duration cycling that are said to occur prior to injury.

\subsection{NON-TRAUMATIC BICYCLE INJURIES}

Cycling, or spinning (stationary biking), is a sport that has a low incidence of acute injury and is considered a low impact, cardio workout (Gregor, Broker, and Ryan 1991; Callaghan 2005). However, cycling makes the rider especially prone to the development of long-term injury due to the repetitive behavior of the workout. While a majority ( $64 \%)$ of acute traumatic injuries occur in cycling competition, it is estimated that more than $90 \%$ of overuse cycling injuries occur in training (de Bernardo et al. 2012). In literature, the cause of these injuries has been theorized to have multiple contributors, such as improper riding technique, poor compensation strategies, and overall muscle fatigue (Boyas and Guével 2011; Cote et al. 2005). The specific long-term injury 
that will be discussed in this thesis is iliotibial band syndrome (ITBS), with potential extensions to patellofemoral pain syndrome, and non-specific lower back pain. Each one of these injuries are common to cyclists and spinners and this study aims to better understand real-time indicators for overuse injury development that can be obtained through repetitive motion and strain on the body (Wanich et al. 2007; Dettori and Norvell 2006).

\section{Iliotibial Band Pain Syndrome}

ITBS is a lateral knee overuse injury in which the iliotibial band (IT-Band, ITB) induces discomfort, pain and in some cases, inflammation [Figure 1-3]. ITBS is common amongst the active population, and is especially prevalent in runners, cyclists, and military personnel (Ellis, Hing, and Reid 2007). ITBS has been speculated to be a kinematic performance issue, where improper technique and subsequent improper loading on the IT band results in pain, with increasing severity over time. Over the years, there has been debate in literature as to what causes ITBS. Many speculate that this injury is a result of friction from the IT band shearing across the lateral femoral epicondyle (LFE) during the extension and flexion of the knee (Farrell, Reisinger, and Tillman 2003; Ellis, Hing, and Reid 2007; Orchard 2007; Hamill et al. 2008). However, the counter arguments cite distal femoral and tibial

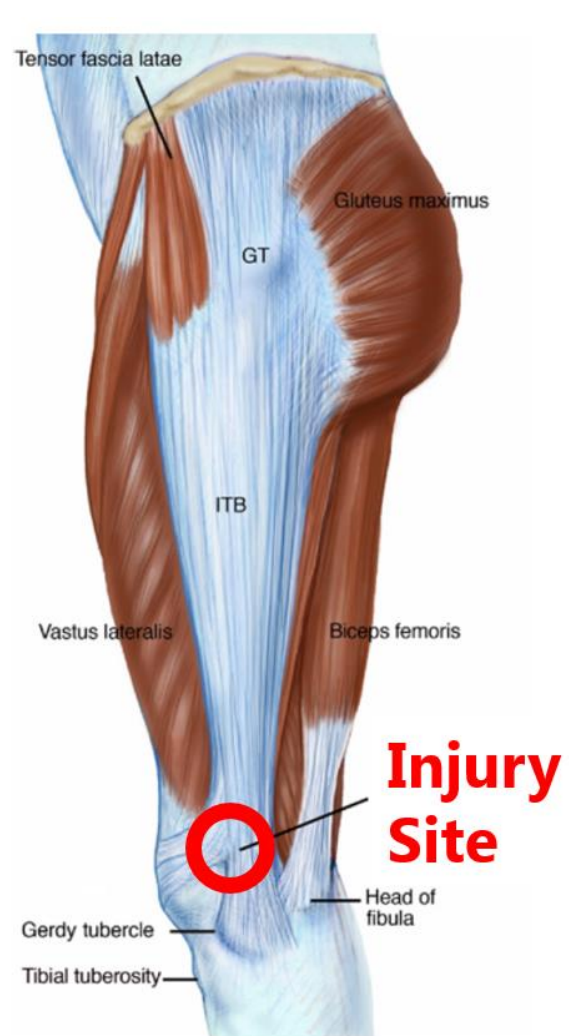

Figure 1-3: ITBS Injury Site [modified from Baker et al.] attachment of the ITB as rendering the IT band unable to rub against the lateral femoral epicondyle in a forward and backward motion. In addition, it is argued that the external appearance of ITB anterior-posterior sliding is an illusion created by a sequential shifting of tensile load within the IT band (Fairclough et al. 2006).

Recent literature, through improved MRI data from symptomatic patients, has shifted the focus of ITBS pathomechanics away from a friction-based injury of the ITB, to one caused by repetitive compression and resulting inflammation of soft-fatty tissue between the ITB and LFE (Flato et al. 
2017; Fairclough et al. 2007). Joint kinematics do contribute to ITBS development, but it is not evident that joint metrics, such as the knee flexion "Impingement Zone" (Farrell, Reisinger, and Tillman 2003), are effective in real-time assessment of injury risk. While the scientific community tends to agree on a compression-based injury mechanism for ITBS, more research is needed on identifying the mechanism(s) of ITBS. Literature for ITBS treatment is also inconsistent; treatment methods range from strengthening the muscles in the surrounding areas, to stretching properly, to over-the-counter analgesics such as ibuprofen, to prescribed corticosteroids (Ellis, Hing, and Reid 2007).

\section{Patellofemoral Pain Syndrome}

Patellofemoral pain syndrome (PFPS) is one of the most common lower extremity injuries of physically active individuals. This condition effects $25 \%$ of active individuals and is particularly prevalent in those who run and cycle (Boling et al. 2009). Boling et al. investigated the risk factors that goes into this understudied condition. Boling et al. found that strengthening of the quadriceps and hamstrings, paired with the understanding and practicing proper technique, can be effective for injury prevention. Proper lower extremity technique to reduce risk of PFPS was found to be decreased hip internal rotation, and increased knee flexion (Malek and Mangine 1981; Boling et al. 2009).

\section{Lower Back Pain}

Non-specific chronic lower back pain (NS-CLBP) is a common disorder amongst cyclists and physically active individuals alike (Van Hoof et al. 2012; Srinivasan and Balasubramanian 2007). Van Hoof et al. investigated the difference in riding posture between riders with and without lower back pain (LBP) by monitoring the flexion patterns of the riders' lumbar spine. It was found that the riders with LBP had significantly more flexion than the healthy rider. It was also concluded that all the riders had increased lumbar flexion from the beginning and that the difference in lumbar flexion between healthy riders and those with LBP stayed consistent (Van Hoof et al. 2012). More research needs to be done on the implications of lumbar strength on LBP prevalence and onset. Given that the posture and technique of the rider seems to have a large influence on their LBP, it could be possible that, similarly to PFPS, there is a need for strengthening certain muscle groups to a specific level before it is safe to cycle long distances with low risk of injury. 


\subsection{OVERUSE INJURY MODELING}

Current methods in long-term injury modeling employ high-dimensional probabilistic algorithms and, more recently, deep learning networks (Pageaux 2014; Tompson et al. 2014; Ji, Lan, and Looney 2006). Methods include effort-based decision models are being developed to draw primarily from cognitive measures, including perceived effort, motivation, perceived time, perceived task progress, and relative task difficulty to training experience (Pageaux 2014). Ji et al. implement 26-parameter static and dynamic Bayesian Networks to predict fatigue. Metrics in such models span temperature, sleep quality, time zone, circadian rhythms, and anxiety (Ji, Lan, and Looney 2006). Without a foundation of longitudinal data available, such methods are often limited in their applicability beyond the task or activity being studied. In applications with extensive userspecific databases, like military personnel training, high-dimensional probabilistic methods have been effective in predicting overall performance on military fitness tests (Springer and Ross 2013; Harman et al. 2008; Lisman et al. 2013). Harman et al. draw from anthropometry and shortduration activity tests (i.e. sit-ups, vertical jumps) to predict battlefield physical performance. For generalization of models to tasks where such data is not readily available, like cycling or stationary biking, alternative methods need to be developed to predict human performance.

\subsection{RESEARCH QUESTIONS}

To address current gaps at the intersection of overuse injury modeling and bicycling fatigue, the conducted investigation focuses on three main research questions:

1) Are an individual's kinematic, kinetic, and physiological parameters correlated over longduration cycling?

2) Can the extent of performance change be modeled with muscle activation metrics?

3) Are ITBS predictors correlated with the extent of performance loss and muscle trends? 


\subsection{RESEARCH OBJECTIVES}

By responding to these questions, this investigation has three objectives:

1) Describe the measured subject parameter changes correlated with performance level.

2) Develop a linear regression model for performance loss based on muscle parameters.

3) Identify the correlation of ITBS predictors with performance loss.

\subsection{METHODS OVERVIEW}

For the purposes of this research, the scope of performance and fatigue modeling has been restricted to high-intensity stationary biking. A comprehensive research approach is implemented within the laboratory setting to provide a framework for future human performance research and device development [Figure 1-4].

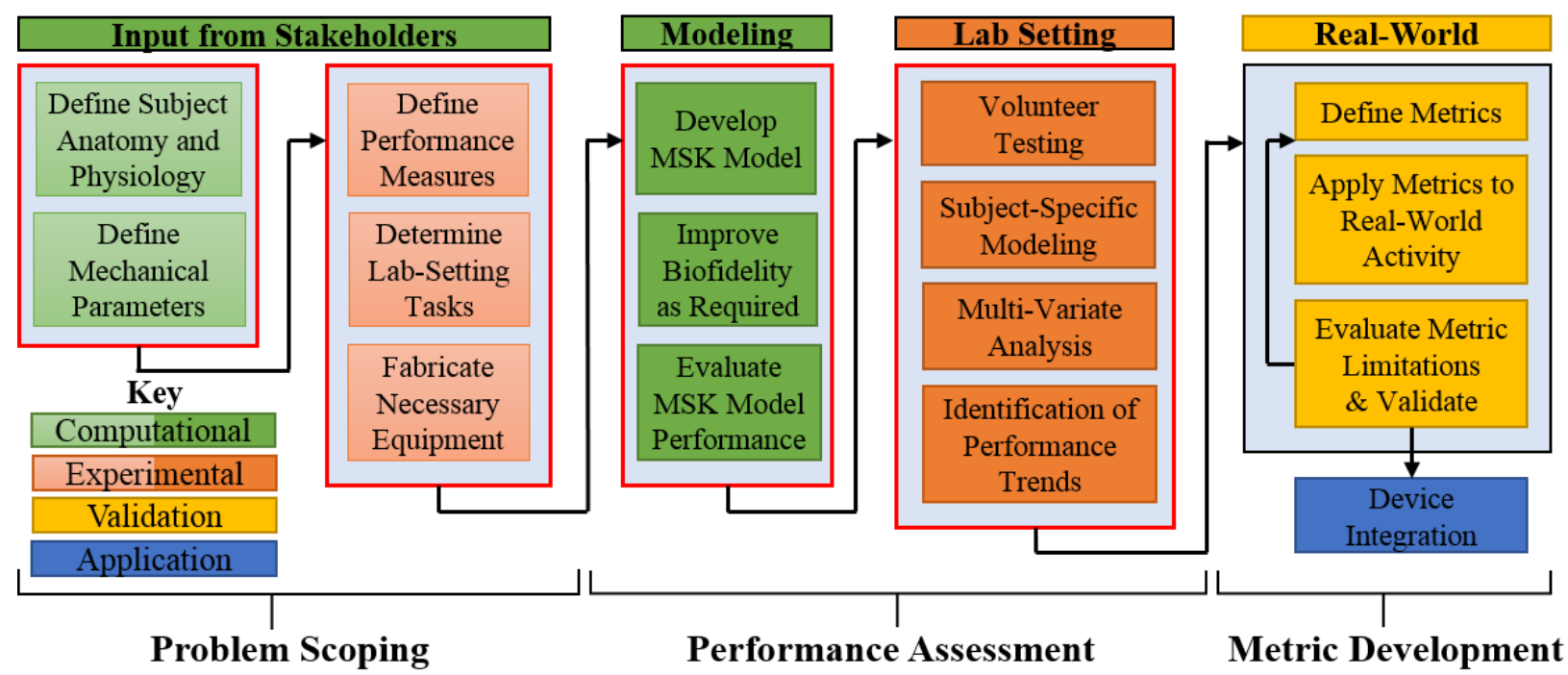

Figure 1-4: Conducted Analytical Framework for Real-Time Human Performance Metrics. Sections outlined in red are implemented in the presented thesis.

Subject kinematics, kinetics, and physiological parameters were collected using three main types of instrumentation. A 9-camera motion capture system (Vicon) was used to measure subject kinematics over the duration of an endurance ride. A commercially available stationary bike was instrumented with 6-axis loadcells in the pedals and seat to collect relevant force and moment data 
at lower extremity boundary conditions. Measured physiology parameters included electromyography (EMG) of abdominal and lower extremity muscles, as well as electrocardiography (EKG). Computational methods (via OpenSim and MATLAB) were used to estimate secondary variables, including osteokinematics, power, efficiency, and LFE compression force. Table 1-1 provides a summary of methods and parameters used through this cycling investigation. Statistical analysis tools were used to test multi-variate correlations and develop fatigue model functions. The following chapters [Chapters 2, 3, and 4] describe in detail the experimental, computational, and analytical methods outlined above.

Table 1-1: Parameters Collected from Endurance Ride

\begin{tabular}{|c|c|c|c|}
\hline & Kinematics & Kinetics & Physiology \\
\hline Collection Method & $\begin{array}{c}\text { 9-Camera Vicon Motion } \\
\text { Capture System }\end{array}$ & $\begin{array}{l}\text { Instrumented } \\
\text { Pedals and Seat }\end{array}$ & $\begin{array}{c}\text { 11-Delsys Trigno Wireless } \\
\text { EMG-EKG Sensors }\end{array}$ \\
\hline & & & $\begin{array}{c}\text { Heart Rate + } \\
\text { Muscle Activity }\end{array}$ \\
\hline $\begin{array}{c}\text { Primary Variables } \\
\text { Collected }\end{array}$ & $\begin{array}{l}\text { Global Coordinates for } \\
\text { Body Segments and Bike }\end{array}$ & $\begin{array}{l}\mathrm{F}_{\mathrm{x}, \mathrm{y}, \mathrm{z}} \text { and } \mathrm{M}_{\mathrm{x}, \mathrm{y}, \mathrm{z}} \\
\text { for Pedals and Seat }\end{array}$ & $\begin{array}{c}\text { Rectus Femoris } \\
\text { Medial Gastrocnemius } \\
\text { Tibialis Anterior } \\
\text { Biceps Femoris }\end{array}$ \\
\hline \multirow{2}{*}{ Computed Variables } & $\begin{array}{l}\text { - Pedal Position } \\
\text { - Pedal Orientation }\end{array}$ & $\begin{array}{l}\text { - Center of Pressure } \\
\text { - Power Output }\end{array}$ & $\begin{array}{l}\text { - Filtered EMG Signal } \\
\text { - Frequency Shift }\end{array}$ \\
\hline & $\frac{\text { From Opensim }}{\text { Osteokinematics }}$ & $\frac{\text { From OpenSim }}{\text { Joint Moments }}$ & $\underset{\text { Optimized EMG Profile }}{\text { OpenSim }}$ \\
\hline
\end{tabular}




\section{Chapter 2}

\section{Performance Description}

\subsection{INTRODUCTION}

Over the years, the competitive cycling community has developed a general understanding of kinematic, kinetic, and muscular trends across terrains (So, Ng, and Ng 2005; Rodrigo R. Bini et al. 2016; Srinivasan and Balasubramanian 2007; Hopker et al. 2013). However, only until recent the advent of advanced motion capture technologies, wireless electromyography, and low-profile load cell development, cycling studies often were limited in only being able to reliably collect one or two types of performance data during laboratory-setting cycling sessions (Gilbertson 2008; Abbiss, Peiffer, and Laursen 2009; Lanferdini et al. 2016). By leveraging current experimental modalities, this investigation could measure full body kinematics, lower body kinetics, and cycling relevant muscle parameters throughout the volunteer testing.

The following sections serve to outline the primary data collection phase of the presented study. Topics will include: volunteer selection, volunteer-bike instrumentation, experimental testing, computational methods, and a summary observed performance fatigue and compensation trends.

\subsection{CHAPTER OBJECTIVES}

The objectives of Chapter 2 analyses on performance description are as follows:

1) Address the lack of same-subject tracking of kinematic, kinetic, and physiological metrics in cycling literature. To this end, the study aims to leverage custom low-profile instrumentation and subject-specific computational modeling in a streamlined volunteerbased framework. 
2) Identify fatigue-induced changes corresponding with performance failure and compensation mechanisms. Classification of subject performance is used to compare system-level performance with sub-system changes over the cycling test.

\section{3) Provide insight on the significance of emergent error pathways in the current overuse} injury development schema. This is achieved by tracking known injury metrics during a high intensity task to failure. Metrics tracked for ITBS in cycling include: hip adduction, impingement zone, and estimates of LFE compression force.

\subsection{EXPERIMENTAL METHODS}

\subsubsection{VOLUNTEER SELECTION}

To reduce cycling experience variation among recruitment responses, advertisement was targeted towards three main cycling groups in the Charlottesville, Virginia area. Advertisement materials and recruitment protocols were pre-approved by the University of Virginia IRB-HRS Review Committee (IRB-HSR: 19248).

Inclusion criteria, as advertised, included:

- 2-3 years of Recreational/Competitive Biking Experience

- Inclusively between the heights of 5' 4" and 6' 6"

- Age: 20 - 45 years old

A total of 35 individuals across three teams responded, and the final selection of volunteers was narrowed to 15 potential volunteers using the following exclusion criteria:

- Women with Known Pregnancy

- Novice/Little Biking Experience

- Children, Prisoners, Cognitively Impaired

- Non-English Speaking (To minimize potential risks due to miscommunication)

- Over 250lbs in weight

- Known heart disease (cardiomyopathy)

- Known history of arrhythmias (irregular heart beat)

- Known current or ongoing physical injury

- History of stroke

- Hypertension (high blood pressure)

- Any other health condition that may put a participant at risk. 
Testing protocols were disclosed to the volunteers at least one (1) week prior to requesting consent. Volunteers were formally consented into the study at the time of their testing session at the Center of Applied Biomechanics following a protocol comprehension test. Accounting for scheduling conflicts and data acquisition errors, a total of 13 subjects were tested in this study. An overview of demographics and biking experience of the tested individuals is presented below. Please refer to Appendix A for additional volunteer details (i.e. injury history, bike settings).

Table 2-1: Summary of Volunteer Physical Characteristics

\begin{tabular}{|c|c|}
\hline Parameter & Mean Value (Standard Error) \\
\hline Sex (M/F) & $11 \mathrm{M} / 2 \mathrm{~F}$ \\
\hline Height (in.) & $70.62(3.45)$ \\
\hline Weight (lbs.) & $167(22.15)$ \\
\hline BMI & $23.46(2.09)$ \\
\hline Age (yrs.) & $29.92(9.27)$ \\
\hline Hip-to-Heel Length (in.) & $42.75(2.31)$ \\
\hline
\end{tabular}

Table 2-2: Summary of Self-Reported Competition and Training History

\begin{tabular}{|c|c|c|c|c|c|c|}
\hline \multirow[b]{2}{*}{$\begin{array}{l}\text { Volunteer } \\
\text { Number }\end{array}$} & \multicolumn{5}{|c|}{ Competition Style } & \multirow[b]{2}{*}{$\begin{array}{c}\text { Training } \\
\text { Frequency? }\end{array}$} \\
\hline & College & $\begin{array}{c}\text { Long } \\
\text { Distance }\end{array}$ & Sprint & Triathlon & Mountain & \\
\hline 2222 & --- & $\bullet$ & --- & --- & $\bullet$ & $>2$ per week \\
\hline 2460 & --- & --- & --- & --- & --- & $\mathrm{x}$ \\
\hline 2593 & $\bullet$ & --- & $\bullet$ & --- & --- & $>2$ per week \\
\hline 2639 & --- & --- & $\bullet$ & $\bullet$ & --- & $>2$ per week \\
\hline 2786 & --- & $\bullet$ & --- & --- & --- & Daily \\
\hline 3579 & --- & $\begin{array}{ll}-- \\
--\end{array}$ & $\begin{array}{ll}-- \\
\end{array}$ & $-\cdots$ & $\begin{array}{ll}-- \\
-\cdots\end{array}$ & $>2$ per week \\
\hline 4218 & --- & $\bullet$ & --- & --- & --- & $>2$ per week \\
\hline 5121 & --- & $\bullet$ & $-\cdots$ & $-\cdots$ & --- & Weekly \\
\hline 7592 & --- & $\bullet$ & $\bullet$ & $\bullet$ & --- & --- \\
\hline 7924 & --- & --- & --- & --- & --- & $>2$ per week \\
\hline 8105 & --- & --- & --- & --- & --- & Daily \\
\hline 8437 & $\bullet$ & --- & $\bullet$ & $\begin{array}{ll}-- \\
-\end{array}$ & --- & 2 per week \\
\hline 9452 & $\bullet$ & $\begin{array}{ll}-- \\
\end{array}$ & --- & $\bullet$ & --- & $\mathrm{X}$ \\
\hline
\end{tabular}

" $\mathrm{x}$ " indicates un-reported information. 


\subsubsection{BIKE INSTRUMENTATION}

A commercially available stationary bike (Bladez III) was purchased and instrumented to measure rider cadence and reaction forces at the pedals and seat [Figure 2-1].

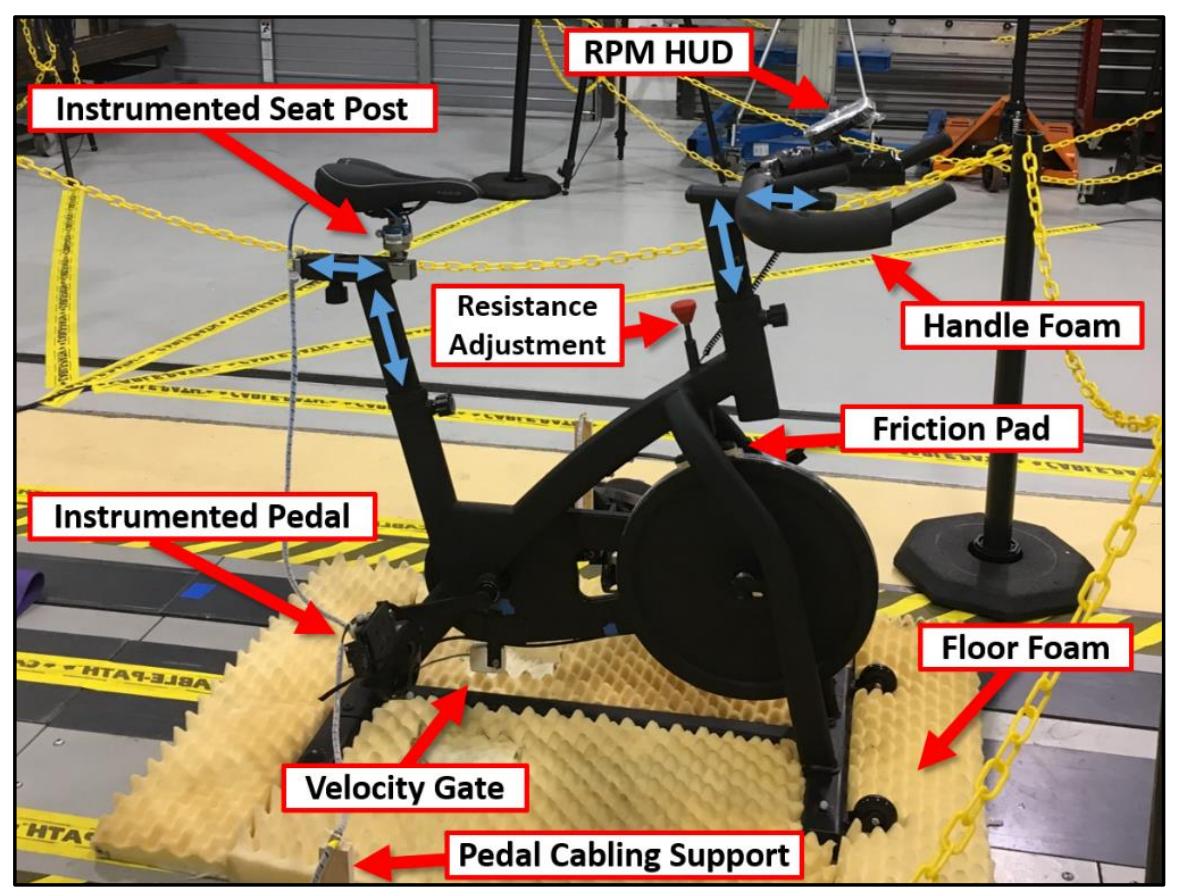

Figure 2-1: Bike Instrumentation Diagram. Blue arrows indicate bike adjustment joint motions.

For cadence, a velocity gate was fixed to the underside of the stationary bike with a metal blade fixed to the distal end of the right crank arm's medial surface. The metal blade was centered at the pedal center of rotation, with a width of $1 \mathrm{~cm}$ and a thickness of $3 \mathrm{~mm}$. At $75 \mathrm{rpm}$ and $1000 \mathrm{~Hz}$ sampling frequency, data acquisition was calculated to sample at least 5-points across the blade; significantly reducing the occurrence of missed or undetected blade passes. Bike also had an integrated cadence (RPM) monitor and output screen for the rider. Upon inspection of the bike, the built-in RPM monitor uses a chain-ring mounted magnet and magnetic field sensor for its calculations. The built-in RPM monitor is used to provide the rider real-time RPM feedback.

Three 6-axis loadcells were used for the pedal and seat kinetics. To minimize pedal profile and mass, a spindle-less pedal bearing (Tioga ${ }^{\circledR}$ Zero-Axel, Figure 2-2) was used to affix the custom pedal to the standard crankarm. Two Humanetics ${ }^{\circledR}$ Q3-ATD Lower-Neck 6-axis loadcells were used in the custom pedal design. The original bike clip was integrated into the custom pedal design and the loadcell origin was centered along the bearing axis-of-rotation [Figure 2-3]. The seat was 
instrumented with a 6-axis implantable femur load cell (low profile) and the seat cushion was replaced with a commercially available high performance-geared seat for rider comfort [Figure 24]. To reduce glare, and subsequent motion capture artifacts, the stationary bike was deconstructed and painted matte black prior to instrumentation. A DTS Slice data acquisition system was used at $1000 \mathrm{~Hz}$ over the entirety of the 67.5 -minute test.

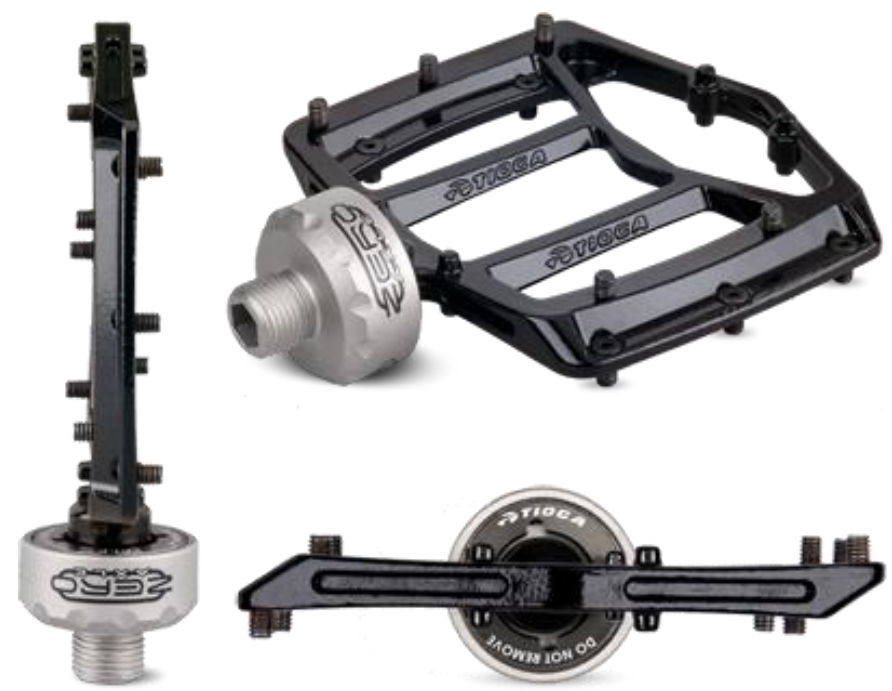

Figure 2-2: Tioga “Zero-Axel” Spindle-less Pedal Bearing with Tioga Pedal. Pedal not used.

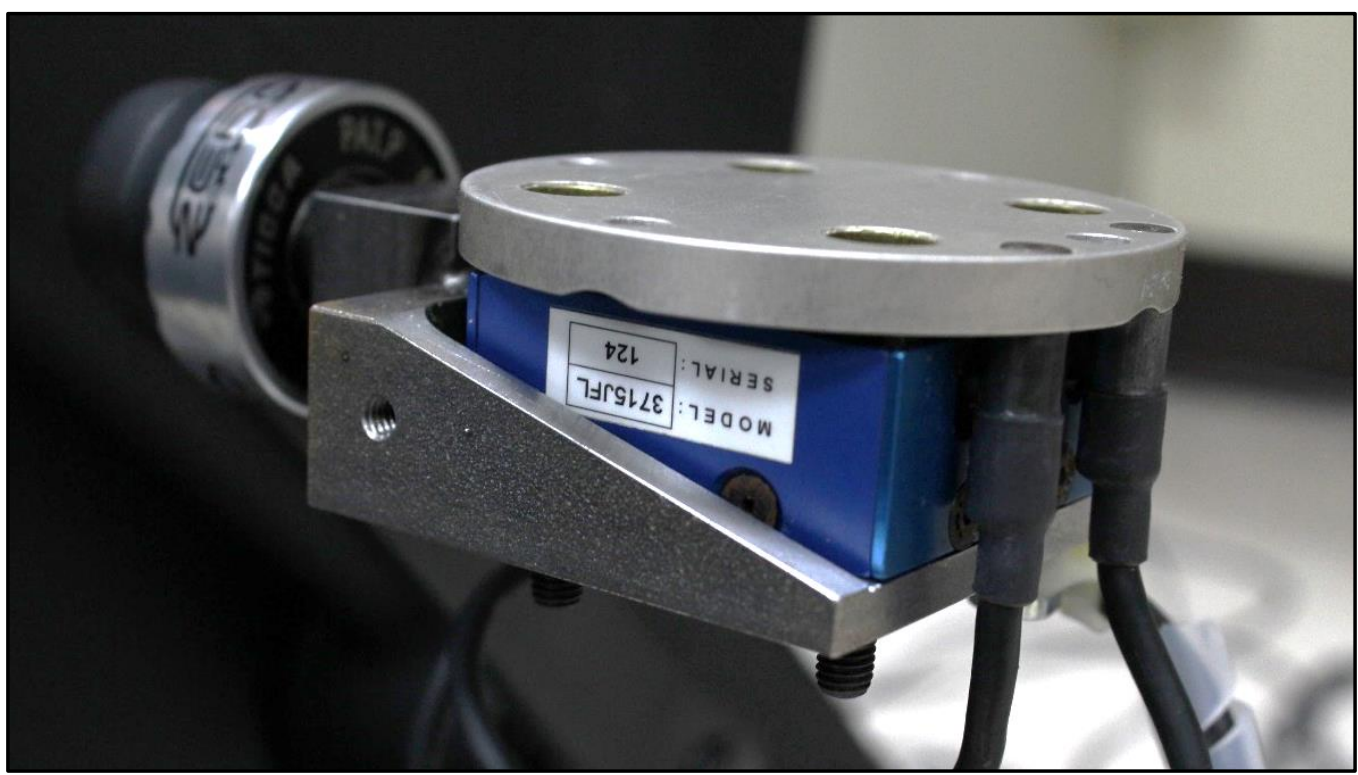

Figure 2-3: Crankarm-Mounted Instrumented Pedal without Foot Plate Harness Attached 


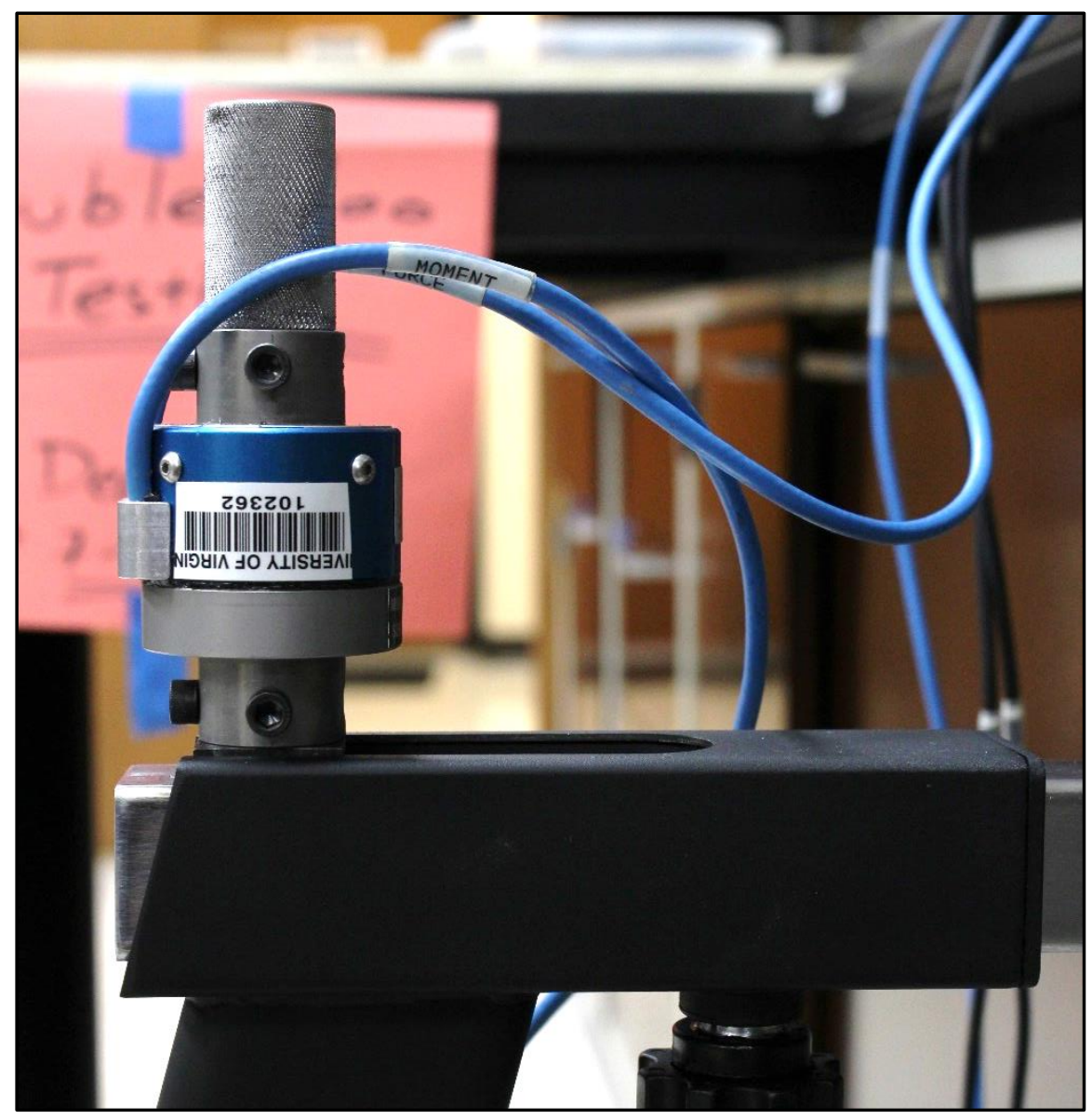

Figure 2-4: Frame-Mounted Instrumented Seat Post without Seat Cushion Attached 


\subsubsection{MOTION CAPTURE}

Kinematic data was collected during the test using a 9-camera Vicon ${ }^{\circledR}$ motion capture system centered around the volunteer biking setup [Figure 2-5]. Five T-10 and four T-20 Vicon cameras were used. A total of 63 retroflective markers were used in each test session: 46 markers to track volunteer body segment motion and 17 for bike pedal, seat, and frame motion. Vicon was run at a $200 \mathrm{~Hz}$ sampling frequency over the entire test (max 67.5 minutes), with a minimum of two cameras viewing a marker to initiate marker trajectory tracking. Reference video was collected using an anterior-oblique GoPro Hero 3+ camera over the duration of test.
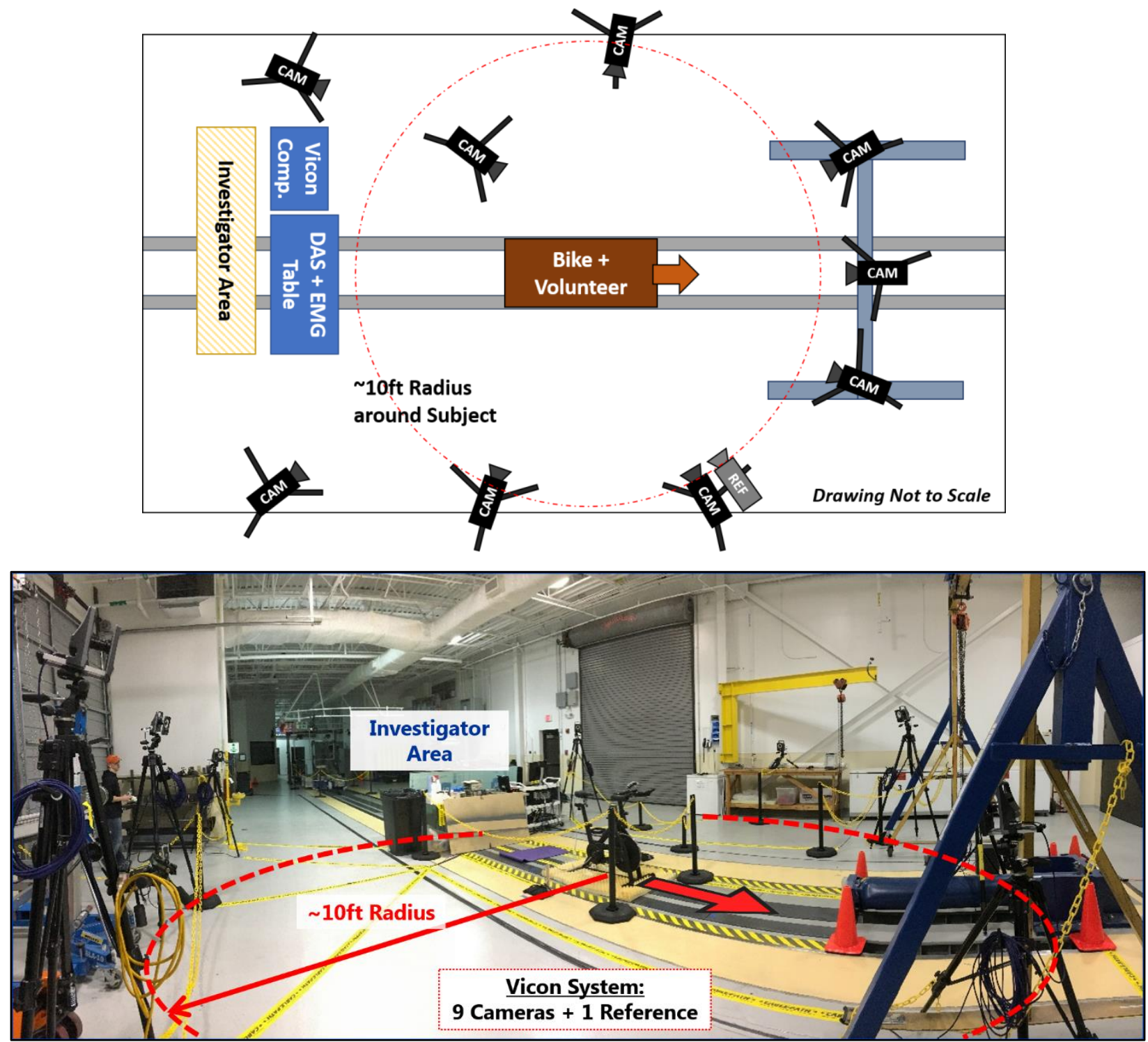

Figure 2-5: Vicon system setup for volunteer testing. CAM indicates Vicon ${ }^{\circledR} T-10 / T-20$ camera. REF indicates reference GoPro® camera. Schematic (top) and final setup (bottom). 


\subsubsection{Physiological MeAsures}

Biological parameters recorded during the test include muscle activation patterns (EMG) and heart rate (EKG). Using a wireless Delsys® Trigno System (Delsys 2014; De Luca 1997), four lower extremity and two core muscles were measured at $1962 \mathrm{~Hz}$. EMG/EKG sensors were wrapped in self-adhesive sports wrap and sweat-resistant tape [Figure 2-6]:

- Rectus femoris (RF - Lower Extremity)

- Biceps femoris* (BF - Lower Extremity)

- Tibialis anterior (TA - Lower Extremity)

- Medial gastrocnemius (MG - Lower Extremity)

- Rectus abdominus (RA - Core)

- Erector spinae (ES, Longissimus - Core)

*Only Right Leg Biceps femoris was successfully collected for all volunteer trials. Bilateral symmetry assumed for fatigue modeling of Biceps femoris. All other muscle signals were measured bilaterally.

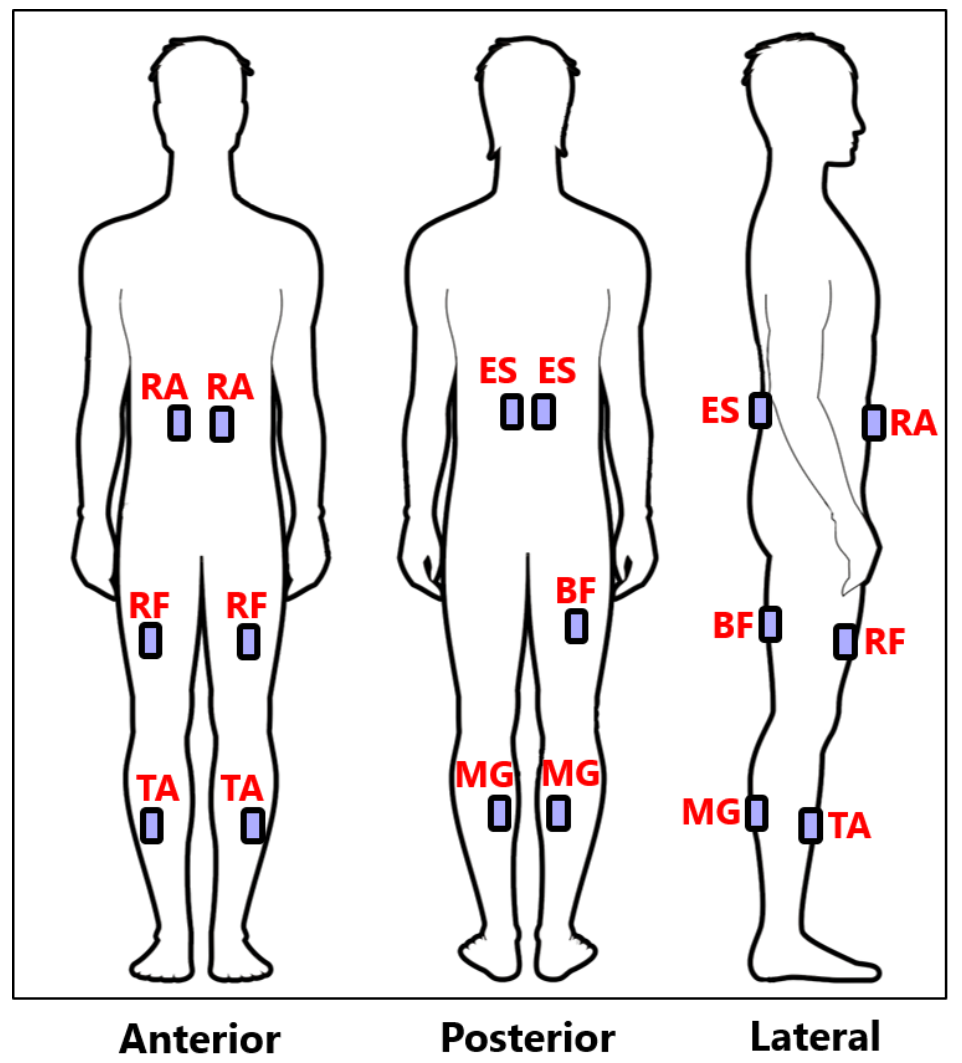

Figure 2-6: Surface EMG Sensor Placement for Volunteer Tests

All data acquisition systems were time-synchronized using a custom trigger box constructed at the Center for Applied Biomechanics. Kinetic, kinematic, and physiological measurement systems were triggered using a 5V TTL signal after a brief warm-up on the bike. 


\subsubsection{VOLUNTEER PREPARATION}

Prior to taking any measurements, volunteers were consented into the study following their arrival to the Center for Applied Biomechanics. To reduce the risk of "no consent" on the testing day, volunteers were run through a pre-screen questionnaire to test comprehension and comfort of the testing protocol. After providing consent, the volunteer was instructed to change into cycling/triathlon shorts (and a sports top for women). A guided floor stretching and warm-up routine was conducted with the volunteer to provide a consistent baseline level of limberness across subjects.

\section{Pre-Ride Stretching Routine}

- 15 seconds of static standing quadriceps stretch

- 15 seconds of static toe touching with one foot over the other (bilateral)

- 15 seconds of static side lunges (bilateral)

- 10 active forward walking lunges (bilateral)

- 10 seconds of static shoulder stretching (bilateral)

- 20 seconds of static sitting straddle

- 15 seconds of static pigeon stretch or preferred equivalent stretch (bilateral)

- 15 seconds of static calf stretch (bilateral)

- 5 minutes of monitored personal stretching

Bottled spring water was provided to the volunteers while retroflective markers and EMG/EKG sensors were affixed with skin-safe double-sided tape. Vicon markers (46) were placed at key anatomical landmarks on the lower extremities, arms, back and head [Figure 2-7, next page].

Following successful attachment of all markers and sensors, the volunteer was assisted onto the bike and oriented on the stationary bike's design and resistance adjustment techniques. Bike adjustments were made to the seat (height, for-aft position) and handle bar (height, for-aft position) using the following guidelines from standard stationary biking sessions:

Seat Height Adjustment Guidelines

- Seat level with standing hip level + minor adjustments for subject's comfort

- Seated with heel on the pedal (6 o'clock) at full leg extension

- Seated with balls of your feet on the pedal (6 o'clock), $145^{\circ}-155^{\circ}$ leg extension

Handle Bar Height Guideline

- Handle bar level with seat + minor adjustments for subject's comfort 
Handle Bar For-Aft Guidelines

- Elbow to second knuckles (proximal interphalangeal joints) should be the distance between the edge of the seat and the handle bar stand

- Slight Adjustments can be made to relieve any discomfort

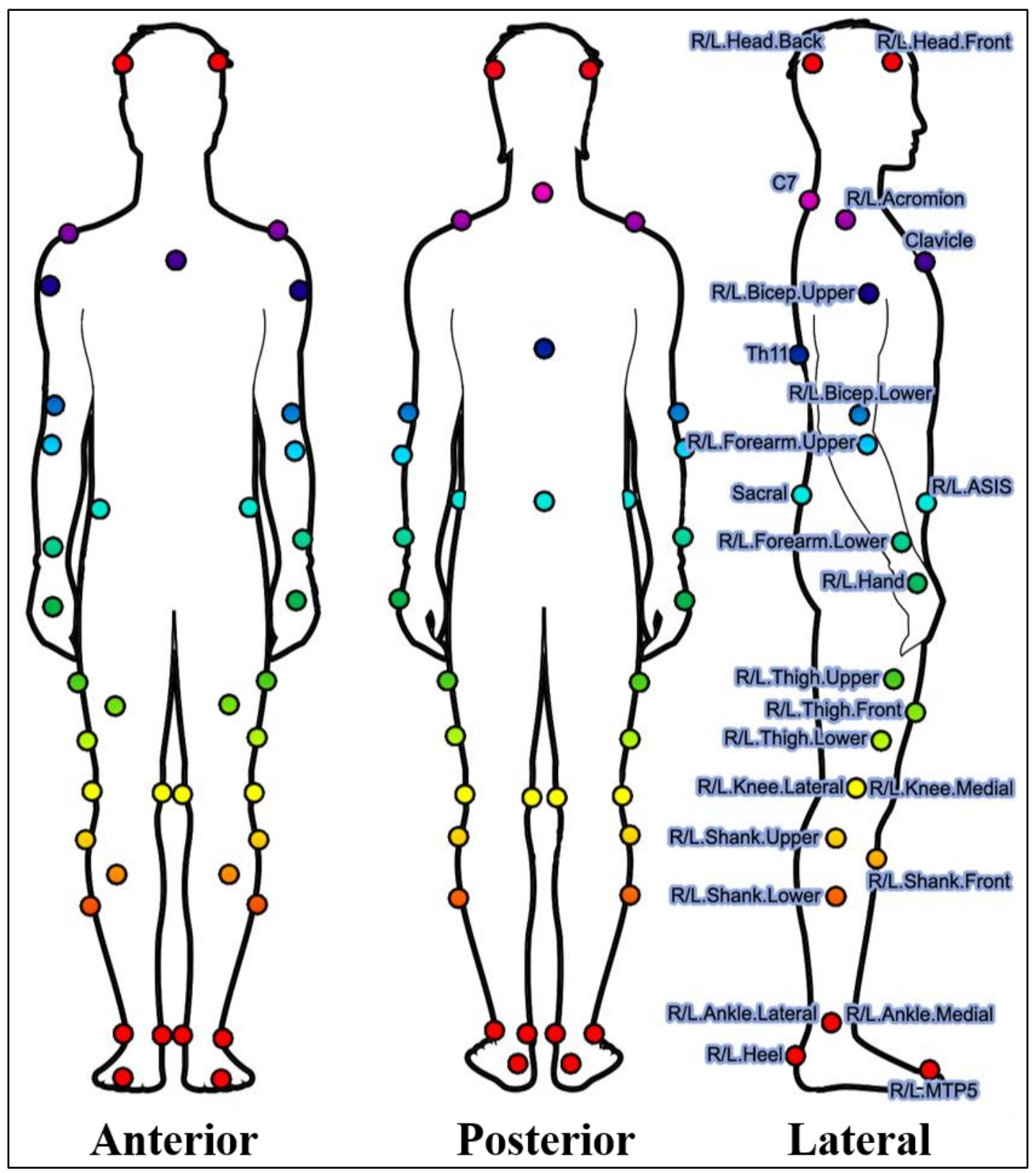

Figure 2-7: Vicon Marker Placement for Volunteer Tests

During bike orientation, a warm-up ride and trigger check were conducted.

Warm-Up Ride Routine

- 2 minutes, low resistance,

- 1 minute, intermediate resistance,

- 2 minutes, low resistance, cadence between 100 and $110 \mathrm{rpm}$ cadence between 90 and $100 \mathrm{rpm}$ cadence between 100 and $110 \mathrm{rpm}$ 
Uninterrupted tracking of motion capture markers on the volunteer and bike was confirmed during the warm-up ride. Figure 2-8 provides a visualization of the complete marker set as seen in the Vicon GUI and post-processing software (Nexus).

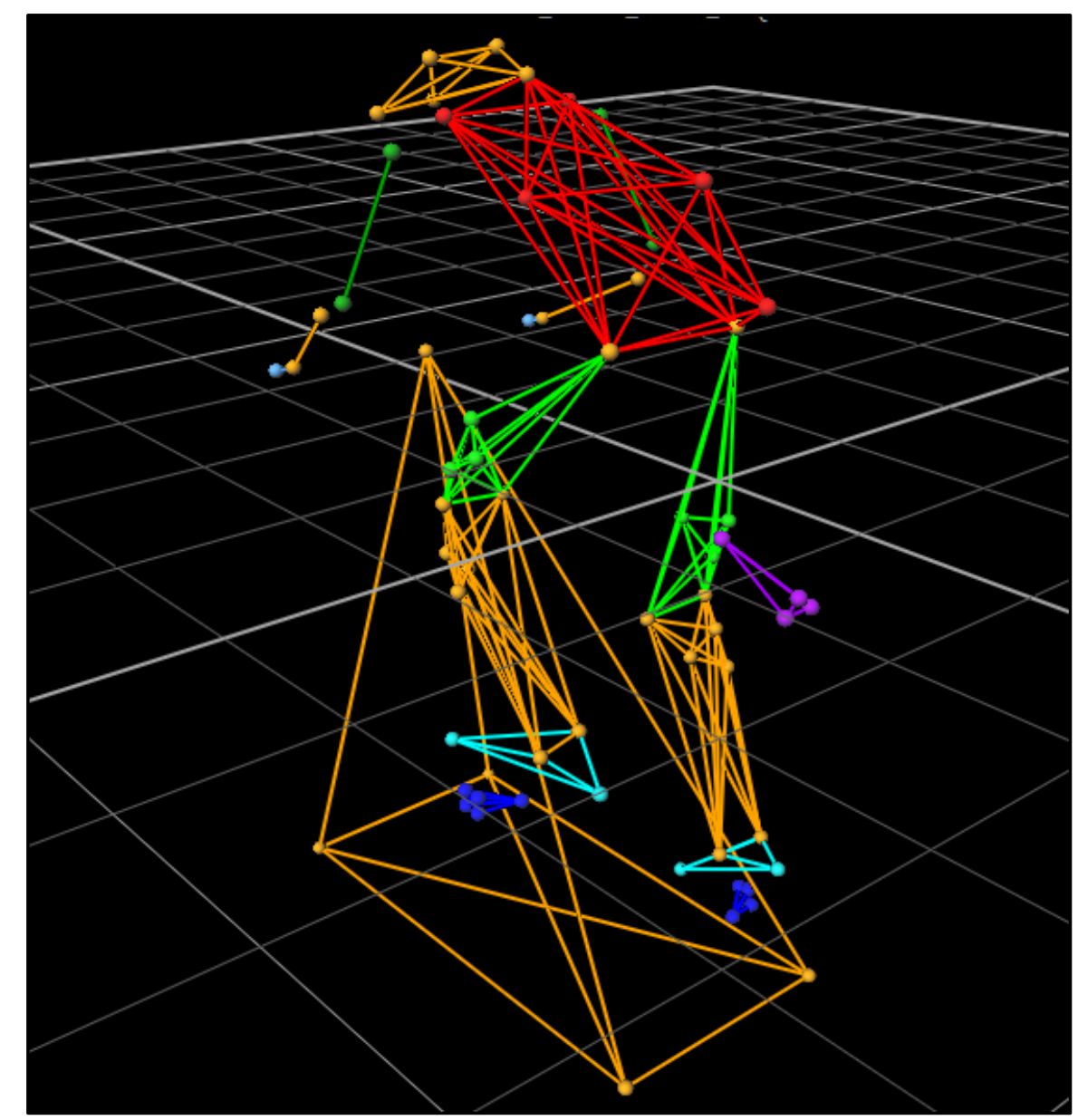

Figure 2-8: Vicon Marker Placements on Volunteer and Bike Segments

After checking successful system triggering and sample data collection across all signals, the volunteer dismounted the bike, sensors were re-zeroed, the volunteer re-mounted the bike, and was then instructed to start low-resistance pedaling to warm-up prior to triggering data acquisition for the main endurance ride. 


\subsubsection{ENDURANCE RIDE ROUTINE}

During the low-resistance warm-up ride, the endurance ride routine was reviewed with the volunteer. Following confirmation of data acquisition system arming, the systems were triggered and the volunteer was guided through the following cycling endurance routine, in which resistance levels are self-selected and are guided by perceived sense of effort [Figure 2-9]:

Endurance Ride Routine

- 2.5 minutes, low resistance,

- 2.5 minutes, intermediate resistance,

cadence between 60 and $75 \mathrm{rpm}$

- 2.5 minutes, high resistance,

- Maintain**, high resistance, cadence between 60 and $75 \mathrm{rpm}$ cadence between 60 and $75 \mathrm{rpm}$ **Attempt to maintain 60-75rpm without dropping below 30rpm

replicating a more realistic training simulation, the use of a constant "sense of effort" routine for cycling decoupled mechanical power output from the perceived discomfort of cycling (Christian et al. 2014). Therefore, mechanical power output during the short duration ride was maximized, and fatigue onset was accelerated (relative to a self-selected constant "perceived difficulty" routine). While without expired gas measurement, perceived intensity resistance settings are considered more relevant than fixed resistance levels (Lanferdini et al. 2016). With training being the primary source of overuse injury development, recreation of such an environment for assessing fatigue was targeted (de Bernardo et al. 2012).

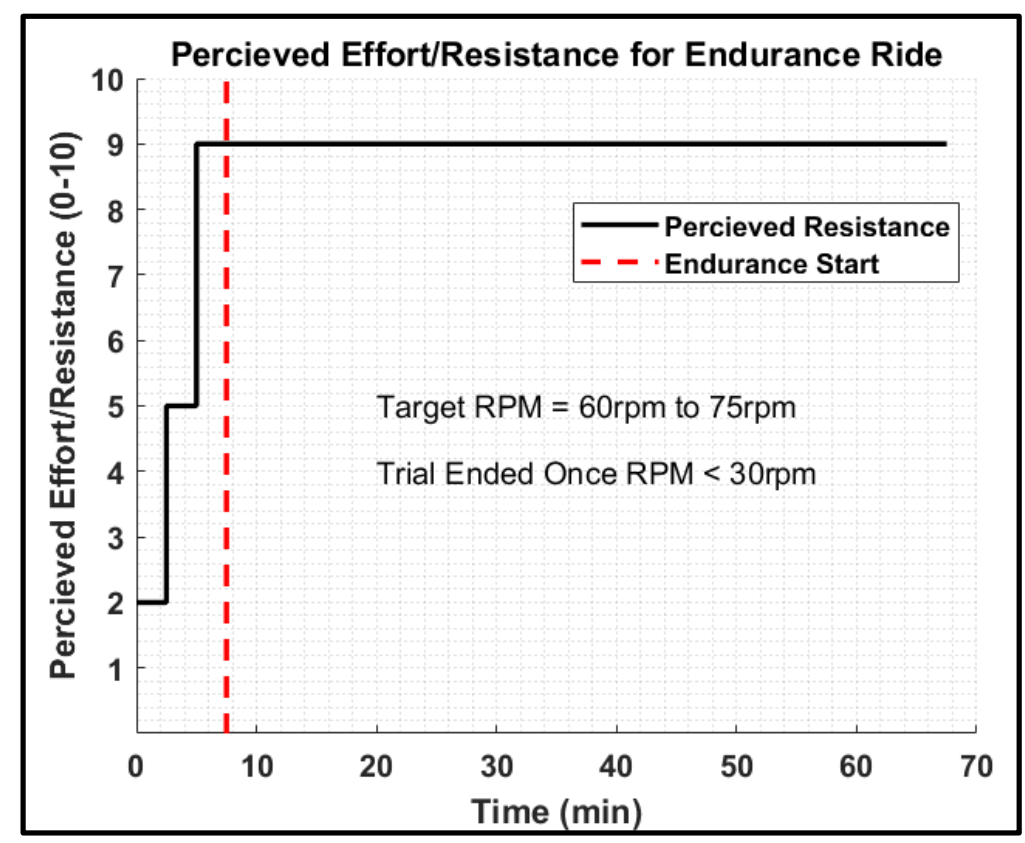

Figure 2-9: Profile of self-selected resistance level over endurance test. Resistance was based on perceived effort. If volunteer reported habituation (perceived effort dropped) to set resistance, the rider was instructed to increase resistance to maintain effort level. 


\subsection{ANALYTICAL METHODS}

\subsubsection{RPM CALCULATIONS}

Three means of cadence (RPM) monitoring were used in this study. For real-time monitoring during the endurance test, the built-in magnetic cadence sensor was used to provide the subject rpm-feedback from their performance. For post-processing, average cadence profiles for each volunteer were found using the velocity gate signal. Thresholding the velocity gate signal and searching for voltage rises resulted in discrete times at which the leading edge of the blade passed the velocity gate, indicating that the right pedal is at its lowest position. Average cadence over a cycle was calculated from the elapsed time between blade passes using the equation below:

$$
\operatorname{rpm}\left(t_{p, i}\right)=\frac{1 \mathrm{rev}}{t_{p, i+1}-t_{p, i} \sec } \frac{60 \mathrm{sec}}{1 \mathrm{~min}}=\frac{60 \mathrm{rev}}{\Delta t_{p, i} \min }
$$

In addition, Vicon markers on the pedals were used to more finely calculate "instantaneous cadence" and pedal velocity over 5-10 second regions of interest. A cluster of four 7.5mm markers were placed lateral to each pedal (centered at the pedal center of rotation) and labeled counterclockwise according to the 4 Cartesian quadrants when the pedal is level with the ground [Figure 2-10].

\section{Right Pedal (Right-Handed)}

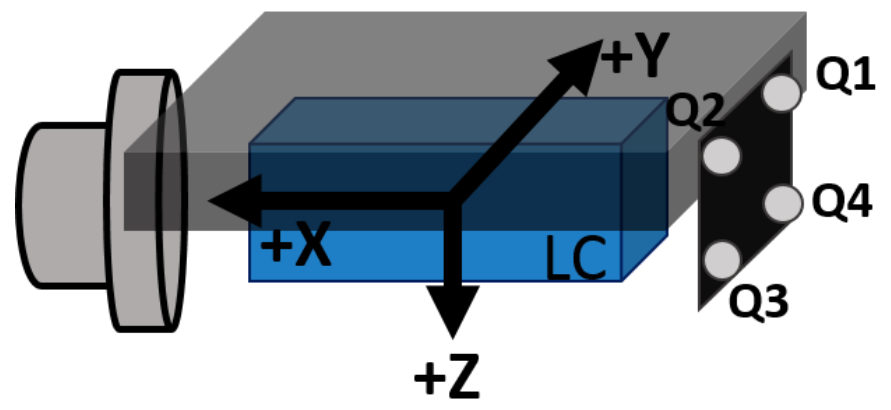

Figure 2-10: Right Pedal Coordinate System and Vicon Marker Cluster 
The pedal's center of rotation (COR, global coordinates) was approximated as the average position of all pedal cluster markers at a given time:

$$
\overrightarrow{\text { Pedal COR }}\left(t_{i}\right)=\left\langle\frac{\sum_{m=1}^{4} Q_{m, x}\left(t_{i}\right)}{4}\left|\frac{\sum_{m=1}^{4} Q_{m, y}\left(t_{i}\right)}{4}\right| \frac{\sum_{m=1}^{4} Q_{m, z}\left(t_{i}\right)}{4}\right\rangle
$$

The crank arm's COR (global coordinates) was then approximated as the average position of the pedal's COR over the selected range of interest. The crank's COR is assumed to be constant over each 5-10 second period.

$$
\overrightarrow{\operatorname{Crank} C O R}=\frac{\sum_{i=1}^{n} \frac{}{\text { Pedal } \operatorname{COR}\left(\mathrm{t}_{\mathrm{i}}\right)}}{n}=\frac{\sum_{i=1}^{n}\left(\frac{\sum_{m=1}^{4} Q_{m, x}\left(t_{i}\right)}{4}\left|\frac{\sum_{m=1}^{4} Q_{m, y}\left(t_{i}\right)}{4}\right| \frac{\sum_{m=1}^{4} Q_{m, z}\left(t_{i}\right)}{4}\right)}{n}
$$

Using the crank arm and pedal COR, a crank arm orientation vector over time was calculated:

$$
\overrightarrow{\text { Crank Orlentatıon }}\left(t_{i}\right)=\overrightarrow{\text { Pedal COR }}\left(t_{i}\right)-\overrightarrow{\text { Crank COR }}
$$

From crank arm orientation vector, crank angle was calculated at each time point and “instantaneous rpm" was found using:

$$
\operatorname{rpm}^{*}\left(t_{i}\right)=\frac{\theta_{\text {crank }}\left(t_{i+1}\right)-\theta_{\text {crank }}\left(t_{i}\right) \mathrm{deg}}{t_{i+1}-t_{i} \sec } \frac{1 \mathrm{rev}}{360 \mathrm{deg}} \frac{60 \mathrm{sec}}{1 \mathrm{~min}}=\frac{\Delta \theta_{\text {crank }, i} 60 \mathrm{rev}}{\Delta t_{p, i} 360 \mathrm{~min}}
$$

With Vicon data collected at $200 \mathrm{~Hz}$, the instantaneous rpm calculation simplifies to:

$$
\operatorname{rpm}^{*}\left(t_{i}\right)=\frac{\theta_{\text {crank }}\left(t_{i+1}\right)-\theta_{\text {crank }}\left(t_{i}\right) \mathrm{deg}}{0.005 \mathrm{sec}} \frac{1 \mathrm{rev}}{360 \mathrm{deg}} \frac{60 \mathrm{sec}}{1 \mathrm{~min}}=\frac{\Delta \theta_{\text {crank }, i} 60 \mathrm{rev}}{\Delta t_{p, i} 1.8 \mathrm{~min}}
$$




\subsubsection{POWER CAlCUlations}

To avoid dependence on commercially available bike power meters, kinematic and kinetic data at the pedals were used to calculate mechanical power during the subject endurance ride. Power is qualitatively calculated by:

$$
P\left(t_{i}\right)=\frac{\text { Work }\left(t_{i}\right)}{\Delta \text { Time }}=\frac{\text { Force }\left(t_{i}\right) * \text { Distance }\left(t_{i}\right)}{\Delta \text { Time }}
$$

Force data from the pedals were collected in local coordinates, which translate and rotate with each cycle. Pedal orientation does not factor into total force calculations, but to calculate mechanical power, pedal orientation relative to the crank arm must be found. First, the pedal load cell's origin must be located. A cluster of $4-7 \mathrm{~mm}$ reflective markers were attached to each pedal.

Using external measurements of the cluster relative to pedal elements, the cluster was positioned at the center of rotation for the pedal. The orientation of the cluster was found using the following functions, assuming only sagittal plane motion of the pedal:

$$
\overrightarrow{\text { Pedal Orientatıon }}\left(t_{i}\right)=\frac{\left[\overrightarrow{Q 1}\left(t_{i}\right)-\overrightarrow{Q 2}\left(t_{i}\right)\right]+\left[\overrightarrow{Q 4}\left(t_{i}\right)-\overrightarrow{Q 3}\left(t_{i}\right)\right]}{2}
$$

Using trigonometric identities, sagittal pedal angle was calculated from pedal orientation vectors. Combining crank angle and pedal angle in the following framework [Figure 2-11] allows for transformation of forces applied in the local load cell $\left(F_{P i}\right)$ coordinates to, global coordinates $\left(F_{G i}\right)$ and local crank arm coordinates $\left(F_{C i}\right)$.

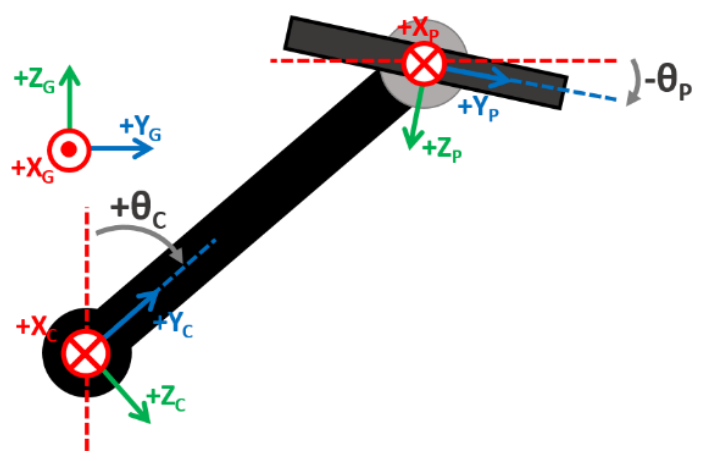

$$
\begin{gathered}
F_{G}=\left[\begin{array}{l}
F_{G x} \\
F_{G y} \\
F_{G z}
\end{array}\right]=\left[\begin{array}{ccc}
-1 & 0 & 0 \\
0 & \cos \left(\theta_{P}\right) & \sin \left(\theta_{P}\right) \\
0 & \sin \left(\theta_{P}\right) & -\cos \left(\theta_{P}\right)
\end{array}\right]\left[\begin{array}{l}
F_{P x} \\
F_{P y} \\
F_{P Z}
\end{array}\right] \\
F_{C}=\left[\begin{array}{l}
F_{C x} \\
F_{C y} \\
F_{C Z}
\end{array}\right]=\left[\begin{array}{ccc}
-1 & 0 & 0 \\
0 & \sin \left(\theta_{C}\right) & \cos \left(\theta_{C}\right) \\
0 & \cos \left(\theta_{C}\right) & -\sin \left(\theta_{C}\right)
\end{array}\right]\left[\begin{array}{l}
F_{G x} \\
F_{G y} \\
F_{G z}
\end{array}\right]
\end{gathered}
$$

Figure 2-11: Right Pedal, Crank, and Global Coordinate Systems and Transformations

Next, displacement of the pedal must be calculated from the available motion capture data. Tracking the pedal's center of rotation with time results in a trace of translational pedal motion. 
Distance translated between time-steps is approximated via the magnitude of the displacement vector between motion capture frames [Figure 2-12].

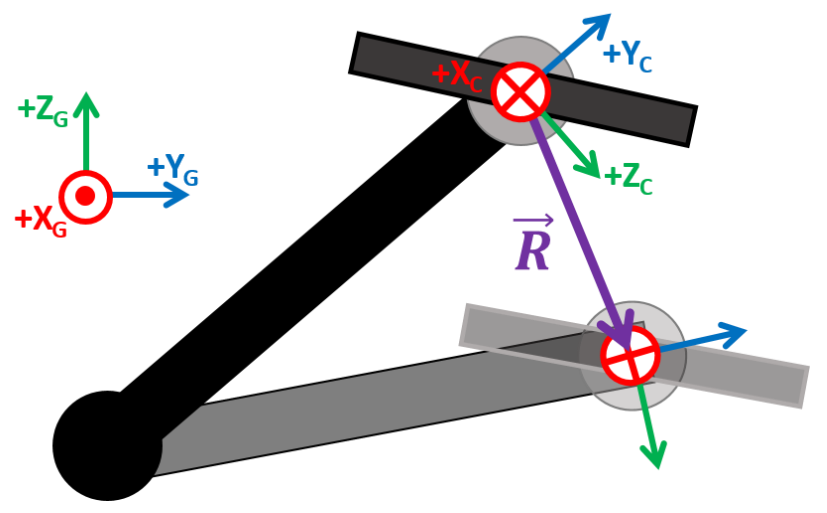

$$
\begin{aligned}
& \vec{R}\left(t_{i}\right)=\overrightarrow{P e d a l ~ C O R}\left(t_{i-1}\right)-\overrightarrow{\text { Pedal COR }}\left(t_{i}\right) \\
& \therefore \text { Chord length }=\left\|\vec{R}\left(t_{i}\right)\right\|
\end{aligned}
$$

Figure 2-12: Displacement Vector Definition for Power Calculations

A more accurate approximation of the distance traveled, especially at high cadence, is arc length:

$$
S\left(t_{i}\right)=\Delta \theta_{\text {crank }}\left(t_{i}\right) *\left\|\overrightarrow{\text { Pedal COR }}\left(t_{i}\right)-\overrightarrow{\operatorname{Crank} \operatorname{COR}}\left(t_{i}\right)\right\|
$$

Using the derived parameters above, total applied force (collected at $200 \mathrm{~Hz}$ ) can be expressed as:

$$
F_{\text {applied }}\left(t_{i}\right)=\sqrt[2]{\sum_{j=1}^{3}\left(\vec{F}_{P j}\left(t_{i}\right)^{2}\right)}
$$

After vector transformation, mechanical power input into the bike is given by:

$$
P_{\text {Mechanical }}\left(t_{i}\right)=\frac{F_{C z} * S\left(t_{i}\right)}{0.005 s e c}
$$

The efficacy of force application was quantified using the "Index of Effectiveness" (IE), defined in literature (Sanderson 1991) as:

$$
I E\left(t_{i}\right)=\frac{F_{C Z}\left(t_{i}\right)}{F_{\text {applied }}\left(t_{i}\right)}=\frac{F_{C Z}\left(t_{i}\right)}{\sqrt{F_{P X}\left(t_{i}\right)^{2}+F_{P y}\left(t_{i}\right)^{2}+F_{P Z}\left(t_{i}\right)^{2}}}=\frac{F_{C Z}\left(t_{i}\right)}{\sqrt[2]{\sum_{j=1}^{3}\left(\vec{F}_{P j}\left(t_{i}\right)^{2}\right)}} \in[-1,1]
$$




\subsubsection{EMG FILTERING AND NORMALIZATION}

Surface EMG data collected during dynamic testing is prone to motion artifacts, electromagnetic interference/noise, and surface impedance variability (Kleissen et al. 1997). To reduce the effect of such noise sources on the EMG signal, a series of pre-processing filtering steps were conducted. From the raw EMG signal, a $60 \mathrm{~Hz} 4^{\text {th }}$-order Butterworth Notch Filter was applied to reduce electromagnetic contamination from the lab environment (Jorge and Hull 1986; Hug 2011). To reduce a broad range of low and high frequency noise from motion artifacts, a $30-300 \mathrm{~Hz} 4^{\text {th }}$-order Butterworth Bandpass Filter was applied (Kleissen et al. 1997; Jorge and Hull 1986; Staudenmann et al. 2010). Collection of all signals was preceded by a zero-input data acquisition to be used for de-meaning the data, thereby removing any initial DC signal offset [Figure 2-13].

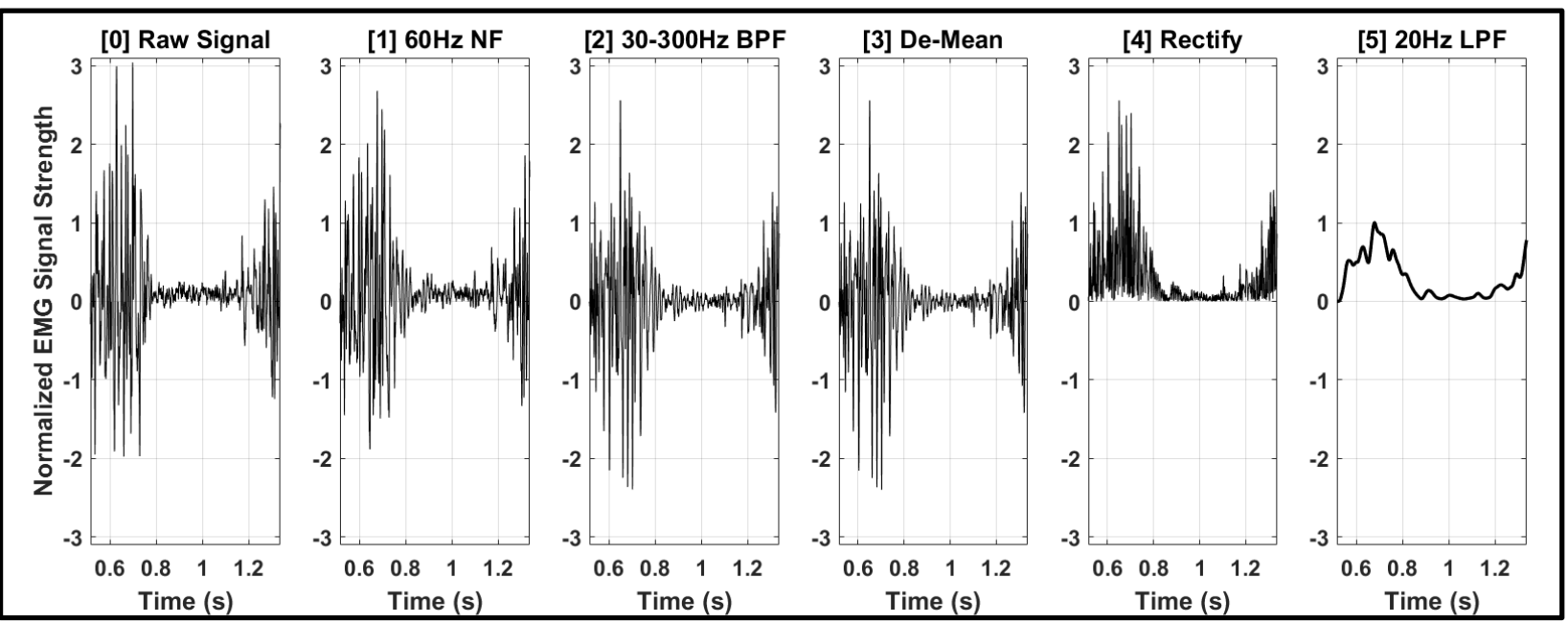

Figure 2-13: Example EMG Pre-Processing Pipeline. Processed from left [0] to right [5]. All presented signals are normalized to maximum value of final $20 \mathrm{~Hz}$ LPF signal. NF $=$ Notch Filter, $\mathrm{BPF}=$ Band Pass Filter, $\mathrm{LPF}=$ Low Pass Filter.

For analysis of the overall activation profile, two additional processing steps were applied. The filtered and DC-corrected data was rectified and run through a $20 \mathrm{~Hz} 4^{\text {th }}$-order Butterworth Low Pass Filter to envelope the EMG signal while preserving mid-frequency data of interest (approximately 1-3x cycling cadence) (Jorge and Hull 1986; De Luca 1997).

Filtered EMG signals for each subject were normalized to allow comparison across subjects and statistical analysis across performance groups. Each muscle signal over the duration of the test was normalized to the average peak electrical signal $(\mathrm{mV})$ during the initial cycling section analyzed. 
The average peak signal was based on peak signals from 25 consecutive cycles. Without MVIC collected for each subject and muscle group, this normalization approach removes systematic signal errors (i.e. skin impedance differences bilaterally and between subjects). The normalized signals are used for all subsequent EMG analyses in Chapter 2 and Chapter 3.

\subsubsection{STATISTICAL ANALYSIS}

Data collected from each volunteer's ride was synchronized in time via trigger box. Each cycle completion was tracked with two methods: motion capture and velocity gate triggering. Given the velocity gate position, times that the right crank was at the $180^{\circ}$ position $(6$ o'clock, lowest position of pedal) could be estimated, but spatial resolution for intermediate positions is limited. Therefore, using the Vicon pedal tracking, each parameter of interest was analyzed in both, temporal (time) and spatial (crank angle) domains. Left side parameters were measured in two spatial reference frames: absolute (left crank angle), and relative (right crank angle). For the analyses presented here, left measures were taken in the absolute crank angle domain, so to allow for concatenation of parameters bilaterally. Central measures (i.e. lumbar flexion, torso/pelvic COM) are presented relative to right crank angle over the cycle. Spatial history of each parameter was prepared over 510 consecutive cycles. Cycling sections were collected at initial, intermediate, and final time points in the trial. Within each cycling section and performance group (described in Section 2.4), parameters are combined into mean \pm standard error corridors over the crank rotation (Eq 13-14).

$$
\text { Mean }_{\text {parameter,section,group }}\left(\boldsymbol{\theta}_{\text {CrankArm,n }}\right)=\frac{\sum_{i}^{N_{s u b}} \sum_{j}^{N_{c y c}} \operatorname{Parameter}_{i, j}\left(\theta_{\text {CrankArm }, n}\right)}{N_{s u b} N_{c y c}}
$$

For the $n^{\text {th }}$ crank angle step, the parameter mean is combined across $N_{s u b}$ subjects and $N_{c y c}$ cycles within each subject's test section. Standard error (SE) was calculated at each crank angle step from similarly concatenated subject-cycle datasets.

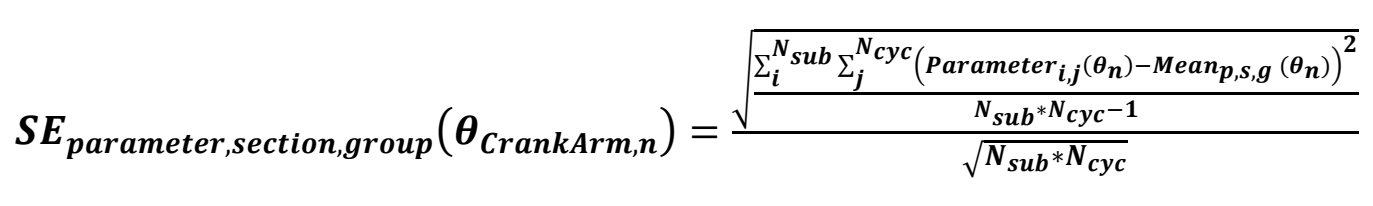


Such spatial history corridors were calculated for the pre-fatigue/initial, intermediate, and fatigue/final time points across volunteers in a performance group. Each parameter corridor point was an aggregate of at least five whole cycles from two legs bilaterally across four subjects within the performance group. Therefore, at least 40 parameter cycles ( 4 subjects $\mathrm{x}>5$ cycles $\mathrm{x} 2$ bilateral) were incorporated into each parameter corridor point across the crank arm rotation domain. Statistically significant differences across cycle sections were evaluated via difference of means Student's t-Test at each crank angle $\left(0.5^{\circ}\right.$ increments) over the $360^{\circ}$ cycle. Significant differences between initial and final cycling sections are presented in the format below [Figure 2-14].

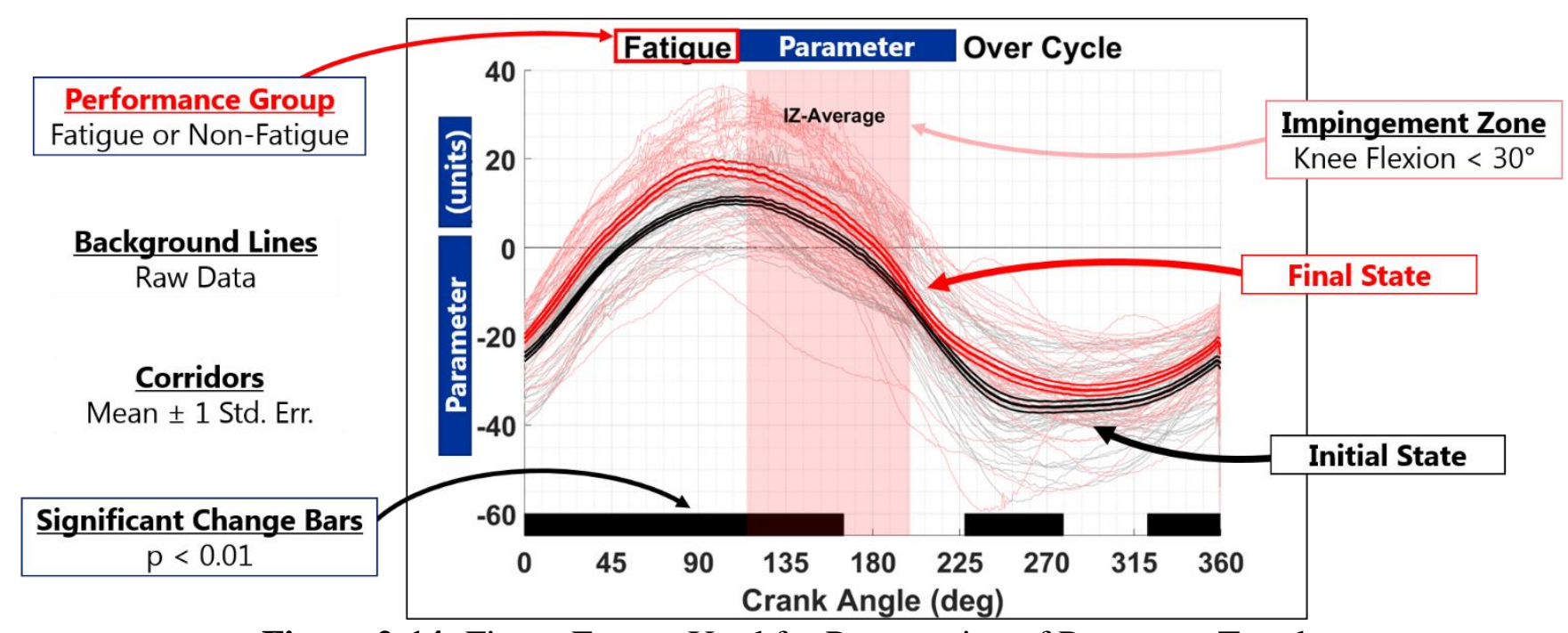

Figure 2-14: Figure Format Used for Presentation of Parameter Trends

For comparison of parameter trends against the literature kinematic ITBS injury risk metric of impingement zone (IZ), knee flexion corridors are analyzed to calculate the average crank ROM when impingement occurs (Farrell, Reisinger, and Tillman 2003) for each cycling section and performance group. Within the "IZ-Average" region (Knee flexion $<30^{\circ}$ ), the IT-band is hypothesized to impinge upon the lateral femoral epicondyle. IZ-Average is presented as a red region in relevant kinematic, kinetic, and EMG figures below. 


\subsection{VOLUNTEER TESTING SUMMARY}

\subsubsection{Data ACQuisition Notes}

During each volunteer test, bike setup was recorded including seat/handle position, resistance adjustment times, and additional comments [Table 2-3 and Appendix A]. Resistance adjustments were made to maintain the perceived effort (resistance level) throughout the endurance ride. Adjustment times (minutes into high endurance ride) are reported in the table below.

Table 2-3: Summary of Test Resistance Adjustments and Comments

\begin{tabular}{|c|c|c|c|}
\hline \multirow{2}{*}{$\begin{array}{c}\text { Volunteer } \\
\text { Number }\end{array}$} & \multicolumn{2}{|c|}{ Resistance Adjustments (min) } & \multirow{2}{*}{ Testing Comments } \\
\cline { 2 - 3 } & Bump Downs & Bump Ups & \multirow{2}{*}{ No Vicon Saved, EMG Restart } \\
\hline 2222 & --- & $22.5,45.5$ & DAS File Corruption \\
\hline 2460 & --- & 53.5 & DAS Truncation after Bump Down \\
\hline 2593 & 10 & 30 & --- \\
\hline 2639 & --- & $20.5,30$ & --- \\
\hline 2786 & --- & $20,35,40,57.5$ & Mistrial (No Data Saved) \\
\hline 3579 & --- & --- & --- \\
\hline 4218 & --- & 18 & Several Markers Lost \\
\hline 5121 & --- & 35 & --- \\
\hline 7592 & --- & 45 & ASIS Marker Standoffs Used \\
\hline 7924 & --- & $17.5,25.5$ & --- \\
\hline 8105 & --- & $8,9.5,10.5$ & DAS Truncation after $3^{\text {rd }}$ Bump Up \\
\hline 8437 & --- & $15,23.5,25,37.5$ & \\
\hline 9452 & --- & & \\
\hline
\end{tabular}

\subsubsection{VOLUNTEER DATA CATEGORIZATION}

Over the 67.5-minute ( $\max$ ) endurance ride, trends were observed based on volunteer performance, as measured through RPM decline. Volunteer performance was categorized into one of three groups: "No Fatigue", "Moderate Fatigue", and "Fatigued/Failure" [Table 2-4]. Using these categories, the volunteers were grouped based on test performance [Table 2-5].

Table 2-4: Observed Performance Types

\begin{tabular}{|c|c|}
\hline Performance Category & Characteristics \\
\hline Non-Fatigue & Maintained greater than 50rpm for entire test \\
\hline Moderate Fatigue & Concluded test with between 40-to-50rpm \\
\hline Fatigue / Failure & Cycled to failure, or less than 35rpm \\
\hline
\end{tabular}


Table 2-5: Volunteer RPM Changes and Performance Types over Trial

\begin{tabular}{|c|c|c|c|c|c|}
\hline \multirow{2}{*}{$\begin{array}{l}\text { Volunteer } \\
\text { Number }\end{array}$} & \multicolumn{3}{|c|}{ Revolutions per Minutes (RPM) } & \multirow{2}{*}{\multicolumn{2}{|c|}{ Performance Type }} \\
\hline & Start RPM & End RPM & $\triangle R P M$ & & \\
\hline 2222 & 68 & 47 & -21 & Moderate Fatigue & - \\
\hline 2460 & --- & --- & --- & $\mathrm{x}$ & --- \\
\hline $2593 *$ & 75 & 44 & -31 & Moderate Fatigue & $\circ$ \\
\hline $2639^{\dagger}$ & 73 & $30^{\dagger}$ & -43 & Fatigue / Failure & $\bullet$ \\
\hline 2786 & 73 & 62 & -11 & Non-Fatigue & $\bullet$ \\
\hline 3579 & 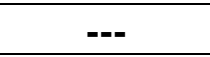 & --- & $-\cdots$ & $\mathrm{X}$ & --- \\
\hline $4218^{\dagger}$ & 79 & $30^{\dagger}$ & -49 & Fatigue / Failure & $\bullet$ \\
\hline 5121 & 68 & 78 & +10 & Non-Fatigue & $\bullet$ \\
\hline $7592^{\dagger}$ & 62 & $30^{\dagger}$ & -32 & Fatigue / Failure & $\bullet$ \\
\hline 7924 & 74 & 60 & -14 & Non-Fatigue & $\bullet$ \\
\hline $8105^{\dagger}$ & 73 & $30^{\dagger}$ & -43 & Fatigue / Failure & $\bullet$ \\
\hline 8437 & 71 & 65 & -6 & Non-Fatigue & $\bullet$ \\
\hline $9452^{\dagger}$ & 67 & $30^{\dagger}$ & -37 & Fatigue / Failure & $\bullet$ \\
\hline
\end{tabular}

"x" indicates volunteer trials with data corruption/loss limitations. *RPM decline before bump down of resistance. ${ }^{\dagger}$ Trial terminated upon reaching 30rpm or failure.

\subsubsection{PERFORMANCE CATEGORY COMPARISON METHODS}

From the performance groups observed, presented analyses pertain mainly to the description and modeling of "fatigue/failure" and "non-fatigue" volunteers. Only two "Moderate Fatigue" volunteers were observed, both of which were removed from analyses due to small sample size. Volunteer characteristics by category are presented [Table 2-6]. Additional parameters for "Hipto-Head Length" and "Leg Length Ratio" were defined, as well $(1,2)$.

"Hip-to-Head Length" = "Height" - "Hip-to-Heel Length"

"Leg Length Ratio" = "Hip-to-Heel Length" / "Height" 
Table 2-6: Summary of Volunteer Physical Characteristics by Performance Category

\begin{tabular}{|c|c|c|c|}
\hline \multirow{2}{*}{ Parameter } & \multicolumn{3}{|c|}{ Mean Value (Standard Error), $\mathbf{n} \mathbf{1 1}$} \\
\cline { 2 - 4 } & Non-Fatigue & Moderate Fatigue & Fatigued/Failed \\
\hline Sex (M/F) & $4 \mathrm{M} / 0 \mathrm{~F}$ & $1 \mathrm{M} / 1 \mathrm{~F}$ & $5 \mathrm{M} / 0 \mathrm{~F}$ \\
\hline Height (in.) & $70.75(3.20)$ & $67.5(2.12)$ & $72.4(2.07)$ \\
\hline Weight (lbs.) & $177.5(10.41)$ & $157.5(10.61)$ & $172.4(15.66)$ \\
\hline BMI & $24.96(1.23)$ & $24.29(0.11)$ & $23.10(1.62)$ \\
\hline Age (yrs.) & $33.5(9.33)$ & $30(12.73)$ & $30.8(9.68)$ \\
\hline Hip-to-Heel Length (in.) & $42.25(1.54)$ & $42.25(0.71)$ & $44.3(1.93)$ \\
\hline Hip-to-Head Length (in.) & $28.5(1.85)$ & $25.25(1.41)$ & $28.1(1.53)$ \\
\hline Leg Length Ratio & $0.60(0.01)$ & $0.63(0.01)$ & $0.61(0.02)$ \\
\hline
\end{tabular}

Participants 2460 (F) and 3579 (M) have been removed from all analyses due to corruption or loss of trial-critical data. Trials from a total of eleven participants resulted in complete datasets that could be analyzed from kinematic, kinetic, and EMG trends. From the "Non-Fatigue" $(n=4)$ and "Fatigue" ( $n=5)$ groups, a series of t-tests were run, testing for significant differences in each volunteer physical characteristics when categorized by performance failure type. The conducted analysis is limited by the low sample sizes, but will provide insight into the potential to use physical metrics to distinguish performance and compensation mechanisms prior to an activity.

Upon closer inspection of data quality, two additional subjects were removed from analysis. Volunteer 7592 data was excluded from the "Fatigue" group due to Vicon marker loss. "NonFatigue" individual 8437 was excluded from the current analysis due to significant Vicon marker loss (due to sweat), and DAS data corruption/truncation. Subsequent analysis includes data of four "Fatigued" and three "Non-Fatigue" participants.

\subsection{PERFORMANCE RESULTS}

\subsubsection{RPM PROFILES}

Over each test session, velocity gate data was collected at the medial-surface of the right crank arm. Each pass of the arm was detected and the time between passes was used to calculate cycling cadence, as described above. For the four volunteers who exhibited performance reduction, the rpm profile over their test has been graphed [Figure 2-15]. For analysis of fatigue-induced 
changes, the final isokinetic region of the test was isolated, the beginning and end of which indicated pre-fatigue and fatigue/failure ( $50 \%$ reduction of $\mathrm{rpm})$, respectively. Due to figure clarity of non-fatigue group RPM, the Isokinetic regions used for analysis of the "Non-Fatigue" group are described in Table 2-7. All test sections used for this performance group exhibited maintained, or slightly increased, RPM.

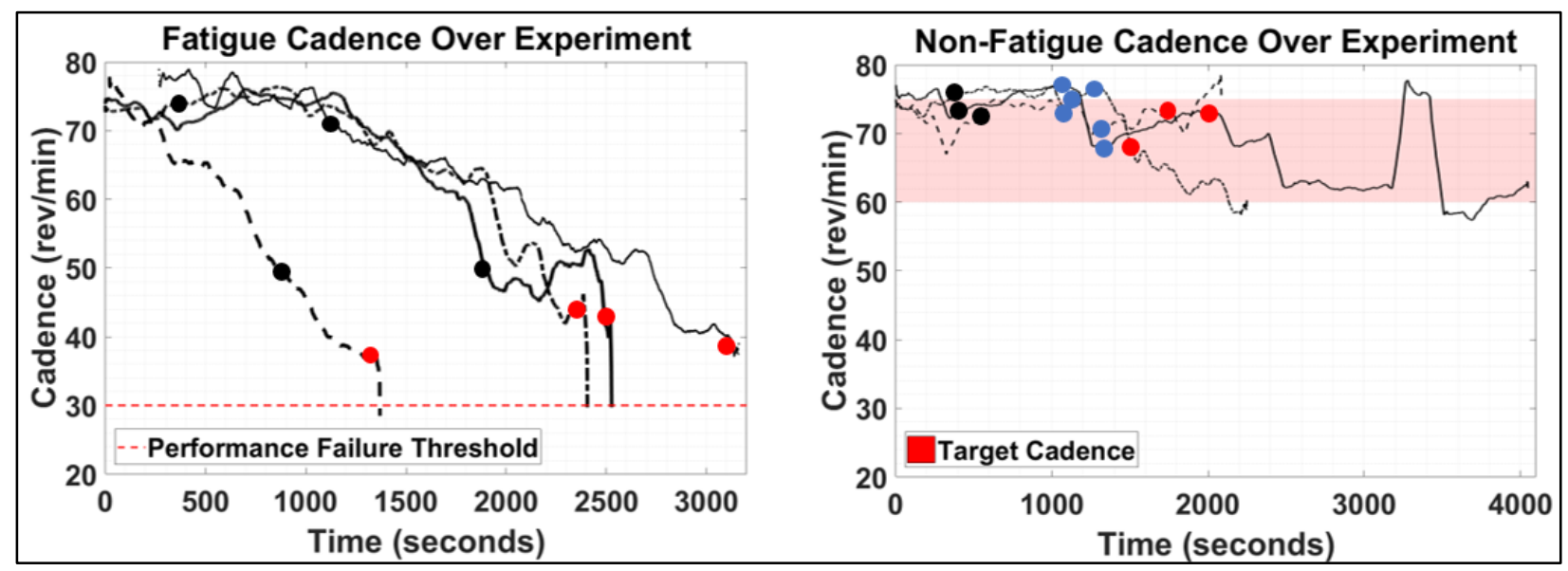

Figure 2-15: RPM Profiles Observed in Endurance Ride. Fatigue subjects exhibited significant performance reduction, while Non-Fatigue subjects maintain performance. Circle markers indicate selected start/pre-fatigue (black) and end/failure (red) states of interest. In the Non-Fatigue group, blue circle markers indicate intermediate points that were analyzed, typically before/after a change in resistance to maintain perceived effort.

Table 2-7: Isokinetic Regions from "Non-Fatigue" Volunteers Used for Performance Analysis

\begin{tabular}{|c|c|c|c|c|}
\hline $\begin{array}{c}\text { "Non-Fatigue" } \\
\text { Volunteer Number }\end{array}$ & Test Region & $\begin{array}{c}\text { Time into High } \\
\text { Resistance (min) }\end{array}$ & Duration (min) & $\begin{array}{c}\text { Average RPM } \\
\text { (Min, Max) }\end{array}$ \\
\hline \multirow{2}{*}{2786} & Isokinetic 1 & 2.500 & 10.000 & $75.3(72,79)$ \\
\cline { 2 - 5 } & Isokinetic 2 & 17.500 & 9.167 & $70.8(66,75)$ \\
\hline \multirow{2}{*}{5121} & Isokinetic 1 & 3.333 & 9.333 & $73.9(67,77)$ \\
\cline { 2 - 5 } & Isokinetic 2 & 15.833 & 6.833 & $71.5(66,77)$ \\
\hline \multirow{2}{*}{7924} & Isokinetic 1 & 2.500 & 8.333 & $76.0(72,81)$ \\
\cline { 2 - 5 } & Isokinetic 2 & 13.333 & 5.167 & $75.6(71,81)$ \\
\hline
\end{tabular}

Isokinetic 2 for these sections is following an increase in resistance to maintain effort level.

Selected subjects for the Fatigue group exhibited significant performance decline, with three of four reaching failure. Analyzed sections from Non-Fatigue subjects did not drop below 60rpm, and two of three subjects increased the set resistance to maintain effort level. 


\subsubsection{MeChaniCAL POWER AND EFFICACY}

Post-processing of bilateral kinetic and kinematic pedal data across fatigued-subjects allowed for comparison of power output and efficiency [Figure 2-16] between pre-fatigue and failure states.
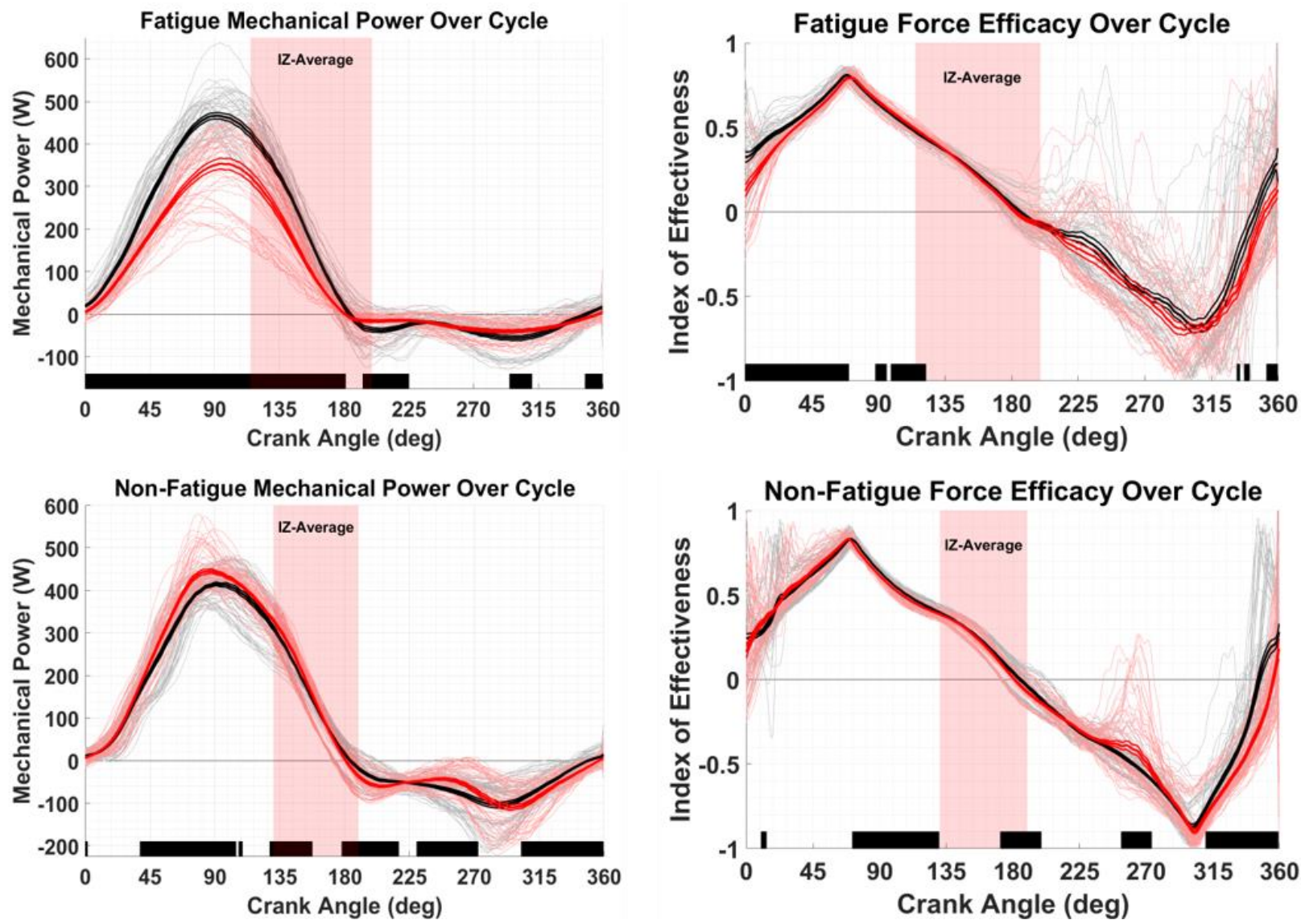

Figure 2-16: Mechanical Power (right) and Force Application Efficacy (left) for Fatigue and NonFatigue Groups. X-axis black bars indicate regions of statistically significant change between initial and final states $(\mathrm{p}<0.01)$. Colors indicate initial (black) and final (red) states of interest.

Statistically significant decreases in net bilateral mechanical power are seen in the Fatigue group between crank angles of $+350^{\circ}\left(-10^{\circ}\right)$ and $+180^{\circ}$, corresponding to the down-stroke. Non-Fatigue subjects exhibit slight power increases during similar regions of the downstroke. During early and late upstroke, slight improvements in power loss are observed in both groups. Fatigue subjects exhibited greater variability in mechanical power output in the last stages of the endurance ride, while Non-Fatigue subjects remained relatively consistent with changing kinetics. Index of effectiveness is seen to follow a similar profile in both groups, positive from late upstroke through to the bottom of the downstroke $\left(+180^{\circ}\right)$. Peak efficacy is consistently observed shortly before peak mechanical power in both performance groups. For a majority of the upstroke, force efficacy 
is negative, indicating "dragging" of the foot as the contralateral leg is generating mechanical power. While the Fatigue group exhibits more positive average upstroke effectiveness, upstroke variability of force effectiveness is consistently greater than that of the Non-Fatigue group.
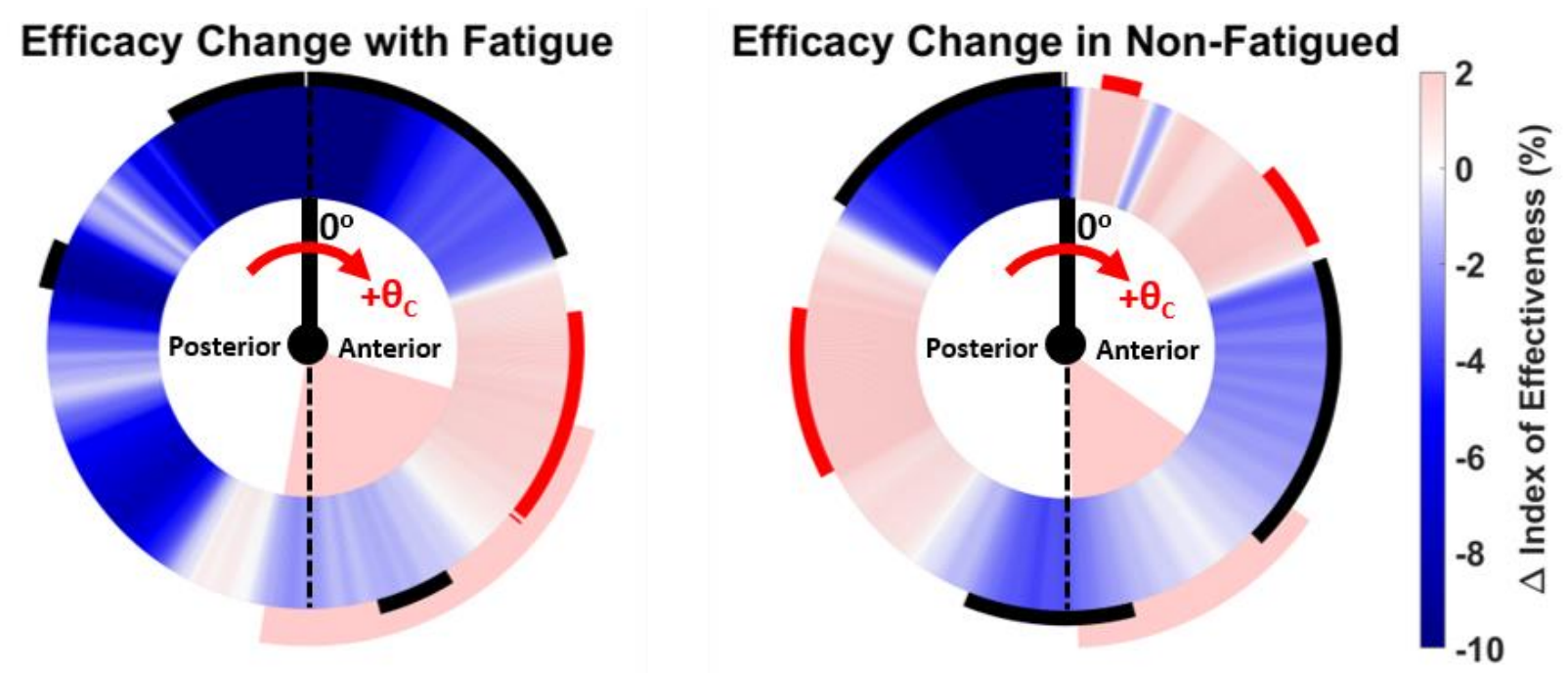

Figure 2-17: Change in Force Application Efficacy for Fatigue and Non-Fatigue Groups. Circumscribed $\mathrm{X}$-axis black bars indicate regions of statistically significant change between initial and final states $(\mathrm{p}<0.05)$. 0o indicates 12 o'clock position of crank arm, with $+\theta \mathrm{C}$ from $0 \mathrm{o}$ defining anterior rotation of the crank arm.

Visualization of efficacy change allows for a closer inspection of the shifts in force application effectiveness with continued cycling and fatigue. In the Fatigue group, down stroke pedal force efficacy exhibits a two-phase change: decreased efficacy (-3\%) during early downstroke, which quickly transitioned to increased force efficacy $(+2 \%)$ during peak mechanical power output $\left(+60^{\circ}-135^{\circ}\right)$. Late downstroke, through upstroke, to early downstroke of the next cycle $\left(+135^{\circ}-\right.$ $60^{\circ}$ ), showed periods of significantly decreased efficacy, with sporadic periods of increased upstroke force efficacy. The Non-Fatigue group efficacy change profile was significantly different. Early downstroke expressed an increased force efficacy $(+2 \%)$, but transitioned to decreased efficacy (-3\%) during peak mechanical power output. In addition, mid-upstroke efficacy increased $(+2 \%)$, corresponding with a significantly less negative mechanical power output. For both performance groups, the top of the cycle $\left(+350^{\circ}-0^{\circ}\right)$ exhibited the greatest decrease in force effectiveness (-10\%) with continued cycling. 


\subsubsection{EMG ACTIVATION PROFILES}

Following EMG pre-processing, the signal envelope is plotted relative to crank angle. Initial/prefatigue, intermediate stages, and final/fatigued EMG signals per muscle are normalized to the maximum initial/pre-fatigue muscle activation signal observed. Left and right leg EMG signals are averaged together to show bilateral muscle activation signals. The four leg muscles analyzed are presented in pairs: "distal leg muscles" of the tibialis anterior and medial gastrocnemius [Figure 2-18], and "proximal leg muscles" of the rectus femoris and biceps femoris [Figure 2-19].

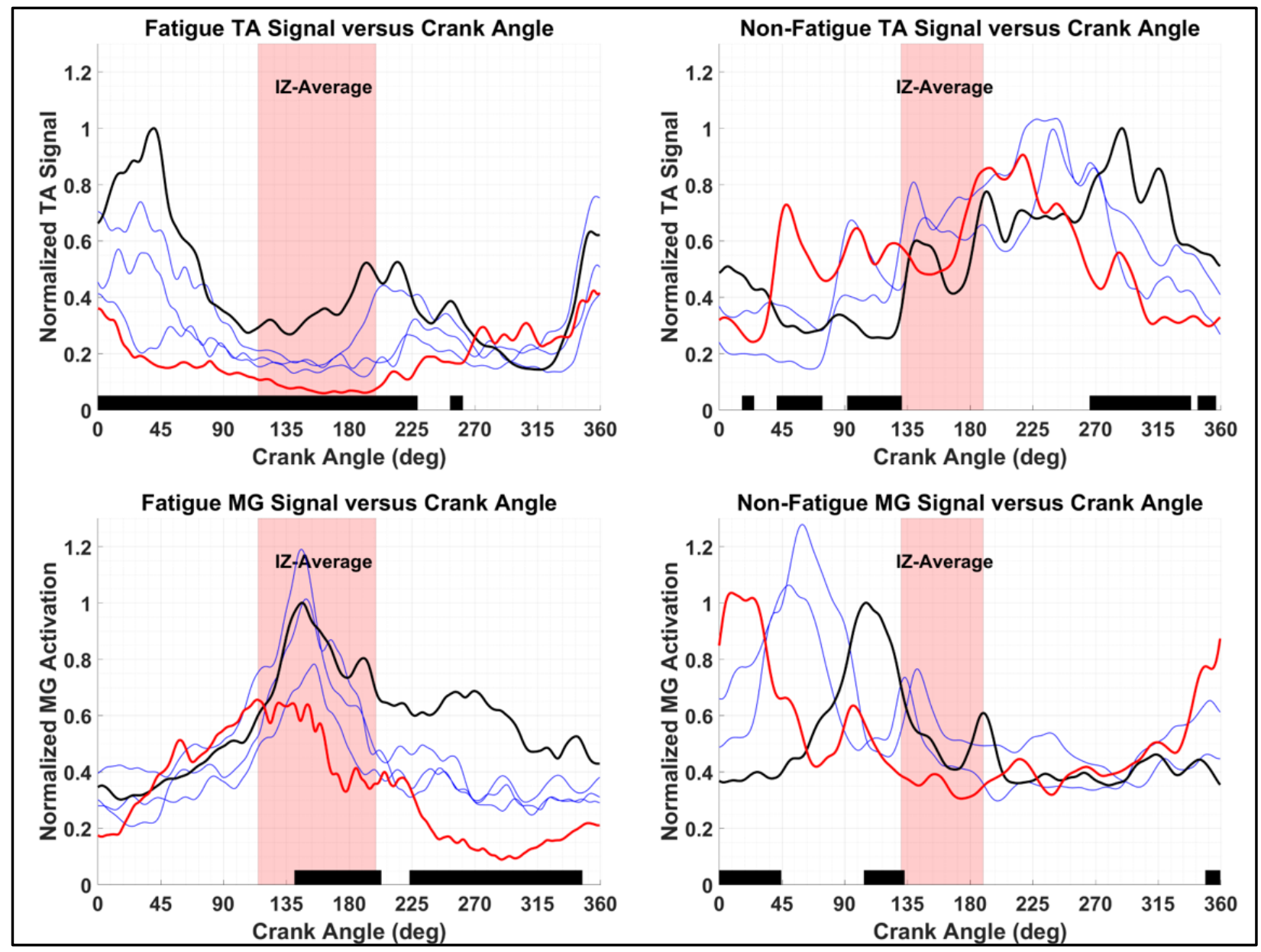

Figure 2-18: Average EMG Profiles for Distal Leg Muscle Groups. Tibialis anterior (top) and Medial gastrocnemius (bottom) for the Fatigue (left) and Non-Fatigue (right) groups at the initial (black), intermediate (blue), and fatigue/final (red) sections of the ride are shown. "IZ-Average" denotes the LFE impingement zone (knee flexion of <30o). X-axis black bars indicate regions of statistically significant change in EMG signal amplitude between initial and final states $(\mathrm{p}<0.01)$.

Both distal leg muscles exhibited significant activation profile changes in the Fatigue and NonFatigue groups. Initial TA activation profile was bimodal in the Fatigue group, peaking at $45^{\circ}$ 
(early downstroke) and $215^{\circ}$ (early upstroke), with a duration of $90^{\circ}$. With continued cycling, the Fatigue group exhibited significant TA signal reduction $(\mathrm{p}<0.01)$ at both activation peaks. Observation of the intermediate activation profiles indicates minimal timing shift of the downstroke activation, but gradual delay of upstroke TA activation (shifting about $90^{\circ}$ from $\sim 215^{\circ}$ to $\sim 300^{\circ}$ ). Final Fatigue TA shows broad low-magnitude activation between $270^{\circ}$ (mid-upstroke) and $30^{\circ}$ (early downstroke), peaking at $\sim 355^{\circ}$ (pre-downstroke). Initial Non-Fatigue TA activation was broad, with activity spanning from $140^{\circ}$ (late downstroke) to $\sim 330^{\circ}$ (late upstroke). Tracking Non-Fatigue TA profile across sections shows insignificant decrease in peak signal magnitude. However, a timing shift is observed, moving peak TA activation $90^{\circ}$ earlier in the cycle (from $\sim 300^{\circ}$ to $\sim 210^{\circ}$ ). Following the timing shift, Non-Fatigue TA is broadly active from $30^{\circ}$ (early downstroke) to $\sim 230^{\circ}$ (mid-upstroke).

Similar analysis of the Fatigue group MG shows initial peak activation at $140^{\circ}$ (late downstroke), with lower broad duration activation from $180^{\circ}$ to $315^{\circ}$ (early and mid-upstroke). At the end of the endurance ride, Fatigue subjects exhibited significantly decreased MG activation during peak activation and the broad activation that followed $(\mathrm{p}<0.01)$. Observation of the intermediate stages indicates the following MG fatigue process: 1) broad upstroke activation decrease and compensatory increase of the pre-upstroke activation, 2) decreased pre-upstroke activation, 3) uniform decrease of MG activity at failure. No gradual changes in activation timing are observed. Initial Non-Fatigue group peak MG activation is centered at $105^{\circ}$ (mid downstroke, spanning $90^{\circ}$ ), followed by a $30^{\circ}$ low-amplitude burst centered at $190^{\circ}$ (early upstroke). Analysis of the NonFatigue group sections indicates maintained activation magnitude, but with a significant $90^{\circ}$ shift of the profile earlier in the cycle (primary peak moving from $110^{\circ}$ to $20^{\circ}$ ). Intermediate stages also reveal an increase in peak MG signal occurring with the timing shift. Non-Fatigue MG profile maintained primary and secondary peak amplitudes in all selected ride sections. 

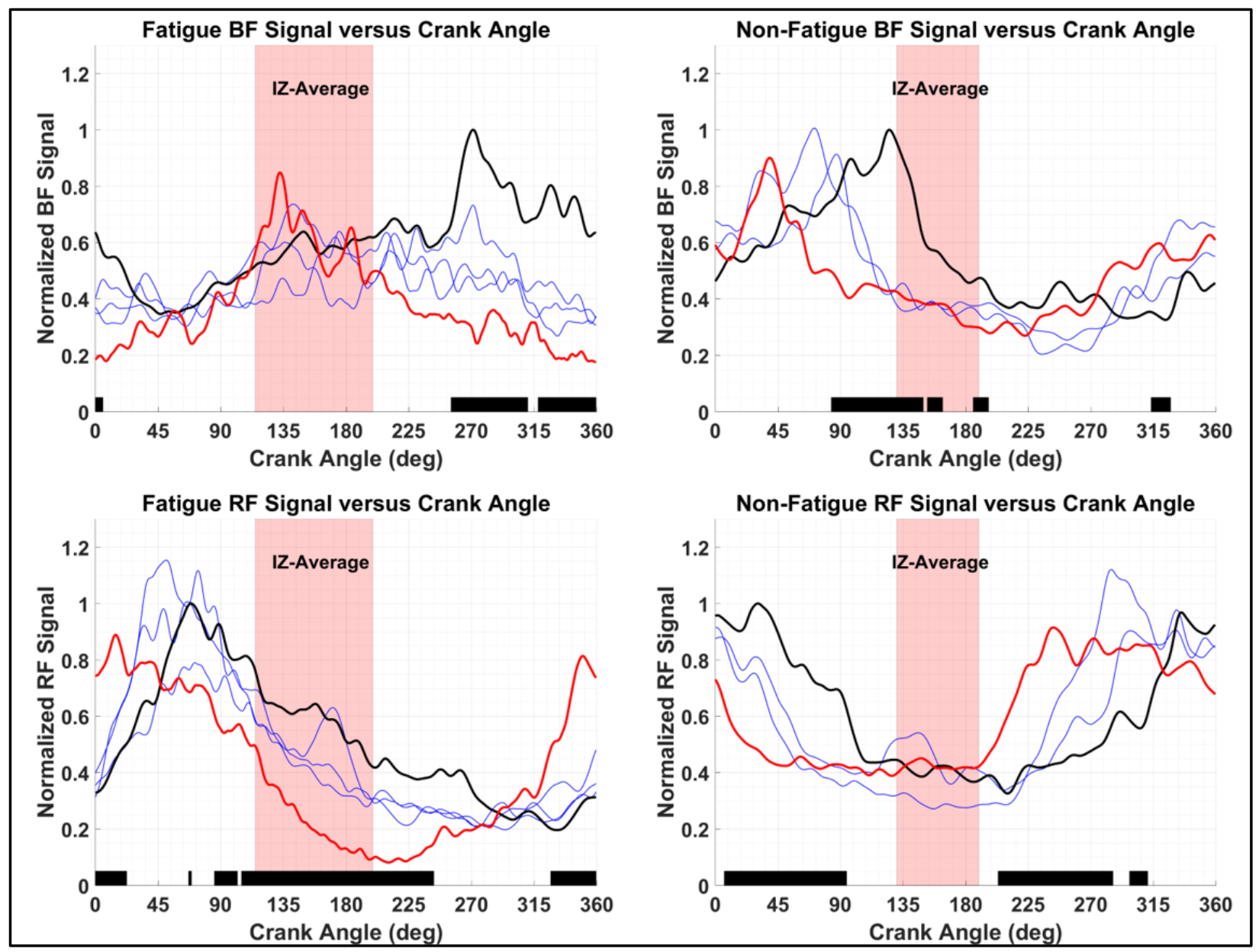

Figure 2-19: Average EMG Profiles for Proximal Leg Muscle Groups. Biceps Femoris (top) and Rectus Femoris (bottom) for the Fatigue (left) and Non-Fatigue (right) groups at the initial (black), intermediate (blue), and fatigue/final (red) sections of the ride are shown. "IZ-Average" denotes the LFE impingement zone (knee flexion of <30o). X-axis black bars indicate regions of statistically significant change in EMG signal amplitude between initial and final states $(\mathrm{p}<0.01)$.

Like the distal muscle group, proximal muscles in the upper leg exhibited significant activation changes in both performance groups. Initial Fatigue group BF profile shows broad moderate-level activity between $135^{\circ}$ (late downstroke) to $20^{\circ}$ (early downstroke) of the following cycle. A peak activation $45^{\circ}$-burst occurs at $270^{\circ}$ (mid-upstroke). At performance failure, the BF signal was found to decrease across the upstroke and early downstroke regime, with significant signal reduction between $250^{\circ}$ (mid-upstroke) and $5^{\circ}$ (early downstroke) of the following cycle. Final Fatigue BF profile exhibited a moderate amplitude peak ( 80\% Pre-fatigue) at $135^{\circ}$ (late downstroke), with a minor secondary peak at $180^{\circ}$. Fatigue group intermediate stages indicate a gradual reduction of mid-upstroke activation while late downstroke activation of the BF increases. Non-Fatigue BF activity shows peak activity centered at $135^{\circ}$ (late downstroke), with moderate 
activity over the downstroke duration (from $15^{\circ}$ to $180^{\circ}$ ). At the end of the section, peak BF activation did not significantly decrease. A timing shift is observed by peak BF activation occurring $90^{\circ}$ earlier in the cycle (moving from $135^{\circ}$ to $45^{\circ}$ ), with a mean decrease in peak amplitude of $10 \%$ from the initial state. Intermediate sections of the Non-Fatigue ride show the time shift of the BF's peak downstroke activation, which also increased late upstroke activation.

Initial Fatigue group RF activation peaks at $60^{\circ}$ (mid-downstroke), with activation ramp-up starting $\sim 355^{\circ}$ (late upstroke) and decreased from $60^{\circ}$ to $200^{\circ}$ (mid-late downstroke). As subject performance declined, significant reduction of downstroke activation is observed. In addition, a shift in activation of $45^{\circ}$ earlier in the cycle (from $60^{\circ}$ to $15^{\circ}$ ) led to a significant increase in predownstroke RF activation with fatigue. Intermediate sections of the ride reveal variability in peak activation concurrent with the activation timing shift. Final Fatigue group RF exhibited a 10\% reduction in mean peak RF activation, while the downstroke activation dropped up to $40 \%$ of the maximum signal. Non-Fatigue subjects initially exhibited a broad maintained activation from $315^{\circ}$ (late upstroke) to $110^{\circ}$ (mid-downstroke). With continued cycling, the RF activation profile had a shift similar to the Fatigue group RF, but moving $100^{\circ}$ earlier in the cycle. Intermediate sections for the Non-Fatigue group also show temporary increase in RF activation while the profile shifted. At the end of the ride section, Non-Fatigue RF maintained initial activation amplitude over a range of $165^{\circ}$ (from $215^{\circ}$ to $10^{\circ}$ of the following cycle). While both performance groups had RF timing shifts, the Fatigue group expressed a smaller RF shift and significantly greater downstroke signal reduction than the Non-Fatigue RF over the analyzed section of the endurance ride.

Cartesian presentation of the EMG signals allowed analysis of the muscle-specific activation trends, but is limited in ease to interpret inter-muscle trends. For this reason, a polar representation of normalized muscle activation signal (Jorge and Hull 1986) was used to qualitatively assess relative muscle activation magnitude, timing, and duration across sections [Figure 2-20]. 


\section{Pre-Fatigue Lower Extremity EMG Signals}

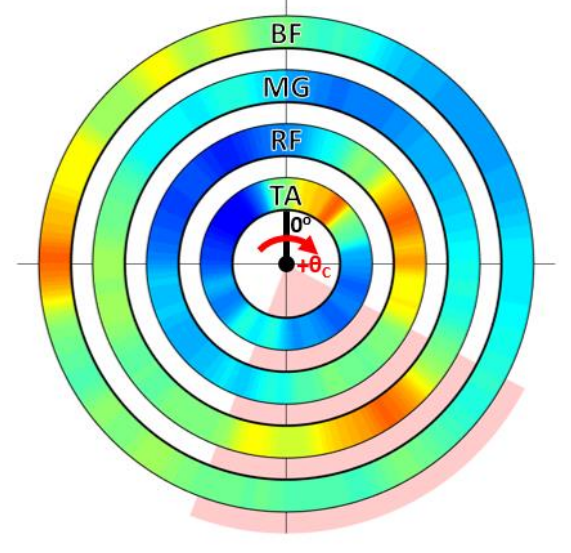

Initial Lower Extremity EMG Signals

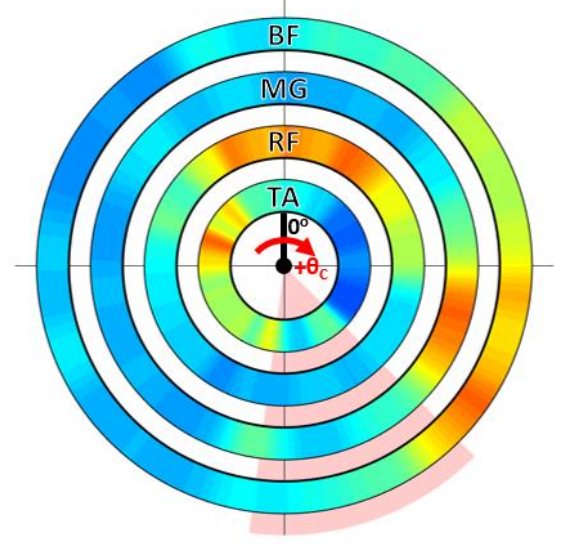

Fatigued Lower Extremity EMG Signals

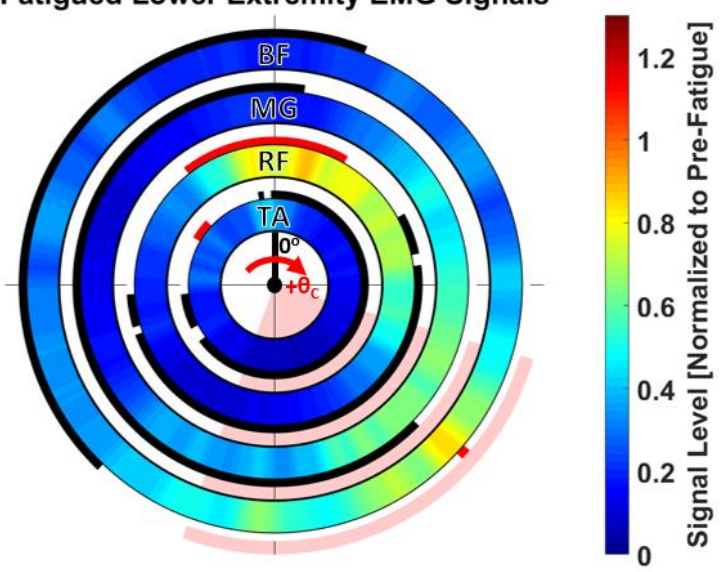

Final Lower Extremity EMG Signals

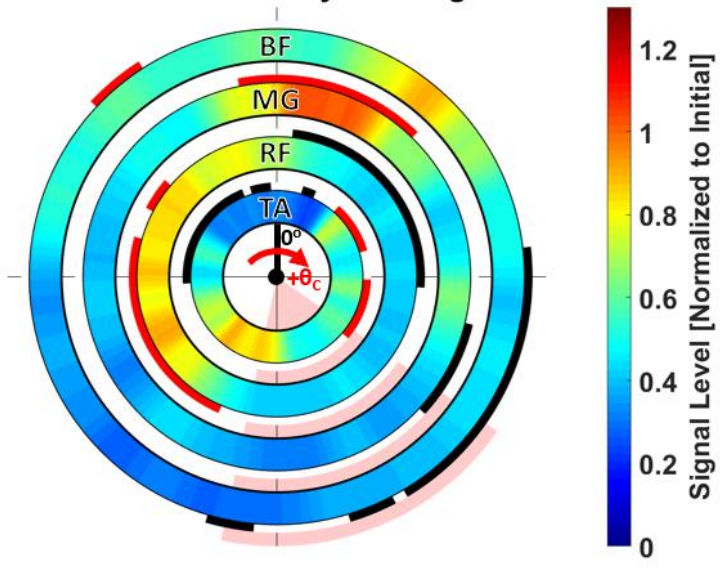

Figure 2-20: Polar Representation of EMG Activation Profiles. Initial (left) and final (right) activation profiles are presented for the Fatigue group (top) and Non-Fatigue group (bottom). Circumscribed black and red bars, respectively, indicate regions of statistically significant ( $\mathrm{p}<$ 0.01) decreases and increases in EMG signal amplitude between initial and final states.

In the initial Fatigue group activation profile, peak TA activation precedes peak RF activation by approximately $20^{\circ}$, and deactivates as RF activation increases. RF maintained broad signal amplitude through late downstroke, decreasing in magnitude as MG and BF activation initiates. MG activation was rapid, reaching peak activation within $45^{\circ}$, while $\mathrm{BF}$ continued at a lowamplitude activation from time of peak MG activation through early upstroke. TA also exhibited a second low-amplitude activation in early upstroke. At mid-upstroke $\left(270^{\circ}\right), \mathrm{BF}$ activation peaked rapidly and maintained a moderate-amplitude activation until completion of the cycle. From BF peak activation, MG continued low activation through to cycle completion. As Fatigue group subjects lost performance, RF activation duration decreases and expresses significantly earlier onset in the cycle, moving into pre-down-stroke regimes. Relative timing between the TA and RF 
was maintained while signal amplitude decreased. BF activation shifts from mid-upstroke to late down stroke, with significant decreases in activation duration and slight variation in intensity. The shifted BF timing established a relative $20^{\circ}$ offset, similar to TA-RF activation.

In the Non-Fatigue group, the activation profile exhibits rectus femoris activation over the top of the cycle, initiating in late upstroke, and continuing through mid-downstroke. As rectus femoris signal decreases, the biceps femoris activates for the remainder of the downstroke. Approximately 20 degrees prior to peak rectus femoris and biceps femoris activation, tibialis anterior and medial gastrocnemius signal peaks, respectively. As the Non-Fatigue group continues cycling, there is a gradual phase shift in the timing of all measured muscle groups. Each muscle group exhibited a 90-degree phase shift, corresponding to earlier mechanical power generation and increased force application efficacy in mid-upstroke and early downstroke. It is interesting to note that the fatigued EMG profile from the Fatigue group approximately aligns with the initial EMG profile of the NonFatigue group. A key difference between these states is that the Fatigue group's tibialis anterior and medial gastrocnemius activation timing does not follow the phase shift seen in the rectus femoris and biceps femoris. Instead, the Fatigue group plantarflexion and dorsiflexion muscles are observed to drop out of the muscle activation cycle. 


\subsubsection{PedAl AND ANKLE KinEMATICS}

EMG analysis of the plantar flexion and dorsiflexion muscles provides insight on the physiological performance of distal muscle groups, but does not show how these activation profiles translate to system-level kinematics. Effective load transfer is dependent on the kinematics at force application surfaces. Analysis of the pedal rotation and ankle flexion trends are used to quantify effective control and range of motion of the distal kinetic chain in cycling [Figure 2-21].

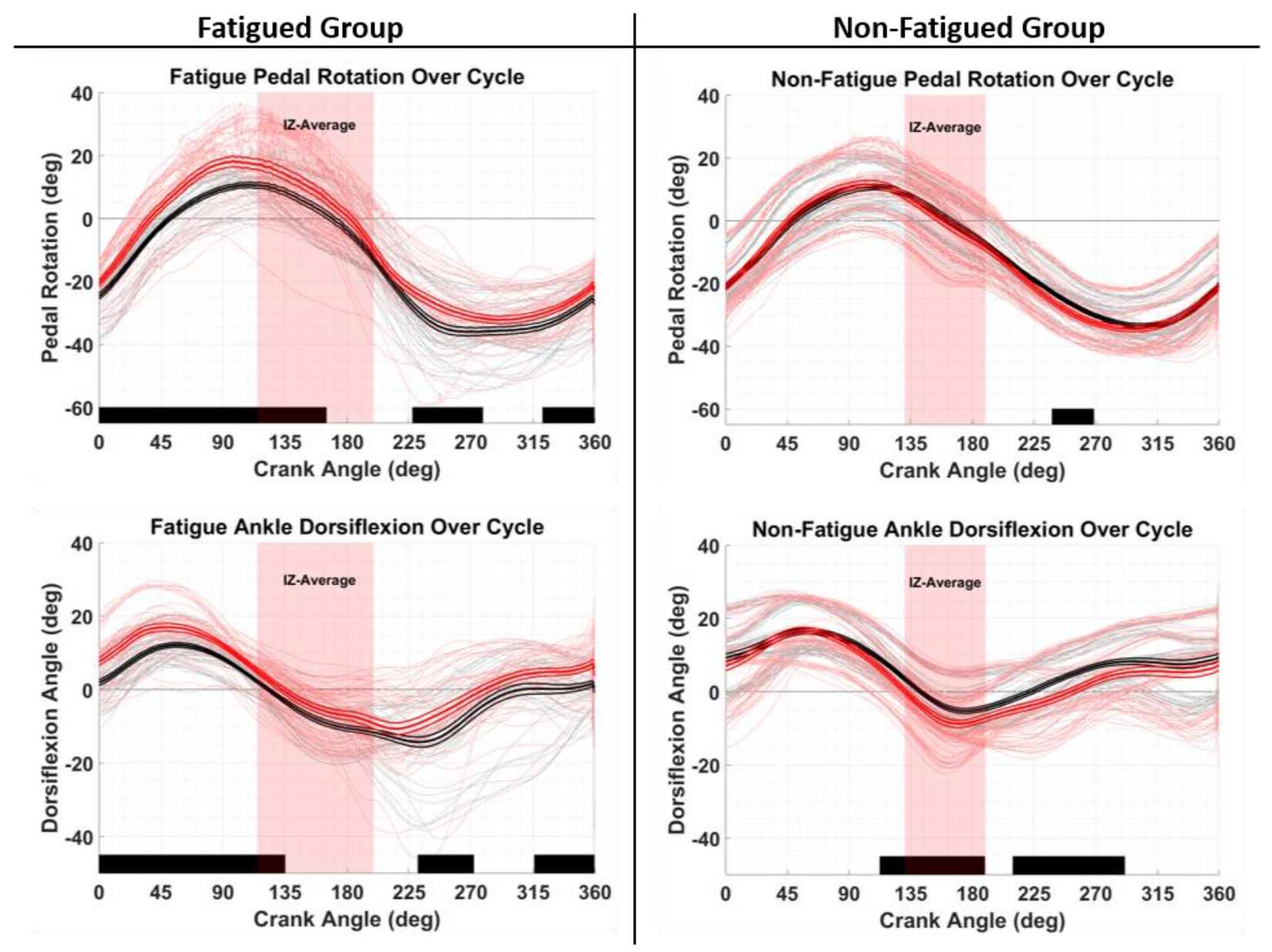

Figure 2-21: Pedal Orientation and Ankle Dorsiflexion Over Cycle During Select Regions of Endurance Ride. Bilateral pedal orientations are shown for the Fatigue (left) and Non-Fatigue (right) Groups. Initial (black) and final (red) sections of the ride are shown. X-axis black bars indicate regions of statistically significant change between initial and final states $(p<0.01)$.

While ROM variability was significantly different between performance groups, the average initial Fatigue and Non-Fatigue pedal rotation profiles were similar. However, the Fatigue group exhibited faster downward pointing of the pedal after initiating the upstroke than the Non-Fatigue 
group, resulting in a longer cycle duration spent in a downward pointing pedal position. Initial ankle kinematics correspond with the pedal observation: Fatigue subjects were on average plantar flexed at the ankle over the cycle, while Non-Fatigue subjects spent more of the cycle dorsiflexed. Both groups start the cycle dorsiflexed, gradually increase dorsiflexion to about $60^{\circ} \mathrm{crank}$ angle (mid-downstroke). For the remainder of the downstroke, the ankle plantarflexed, reaching peak plantar flexion at downstroke completion $\left(\sim 180^{\circ}\right)$. Fatigue subjects remain in this position, increasing plantar flexion slightly at $240^{\circ}$ (mid-upstroke), and return to a neutral position at $300^{\circ}$ (late upstroke). Non-Fatigue subjects, instead, begin dorsiflexing the ankle from upstroke initiation, reaching $+10^{\circ}$ of dorsiflexion by $270^{\circ}$ in the cycle (mid-upstroke).

At the end of the ride section, Fatigue subjects showed significantly more dorsiflexed ankle position $(\mathrm{p}<0.01)$ from pre-upstroke through much of the downstroke $\left(315^{\circ}-135^{\circ}\right)$ and at midupstroke $\left(230^{\circ}-275^{\circ}\right)$. Ankle kinematics in this group directly corresponded with pedal rotation changes of similar magnitude and direction $(\mathrm{p}<0.01)$. Non-Fatigue and Fatigue subjects did not share the timing or direction of ankle osteokinematic changes. In the final section, Non-Fatigue subjects displayed significantly increased plantar flexion during late downstroke $\left(110^{\circ}-180^{\circ}\right)$ and early-to-mid upstroke $\left(210^{\circ}-295^{\circ}\right)$. These ankle changes did not translate to more downward pedal rotation during the same cycle times; only a shortened mid-upstroke region $\left(235^{\circ}-270^{\circ}\right)$ exhibited increased downward pedal orientation. From these results, it is indicated that the ankle control has a significant effect on pedal control in the Fatigue group, while Non-Fatigue subject performance hints that other adjustments up the kinematic chain counteract ankle plantar flexion changes and maintain relative pedal orientation through the ride section.

\subsubsection{OPENSIM KINEMATICS}

Moving up the kinetic chain, the Inverse Kinematics toolset within OpenSim [See Chapter 4.2] utilized Vicon motion capture data allowed to approximate joint osteokinematics for each volunteer. From the joint osteokinematics available in OpenSim IK, knee [Figure 2-22], hip [Figure 2-23], and torso/pelvis COM trajectories [Figure 2-24,25,26,27] are presented. 


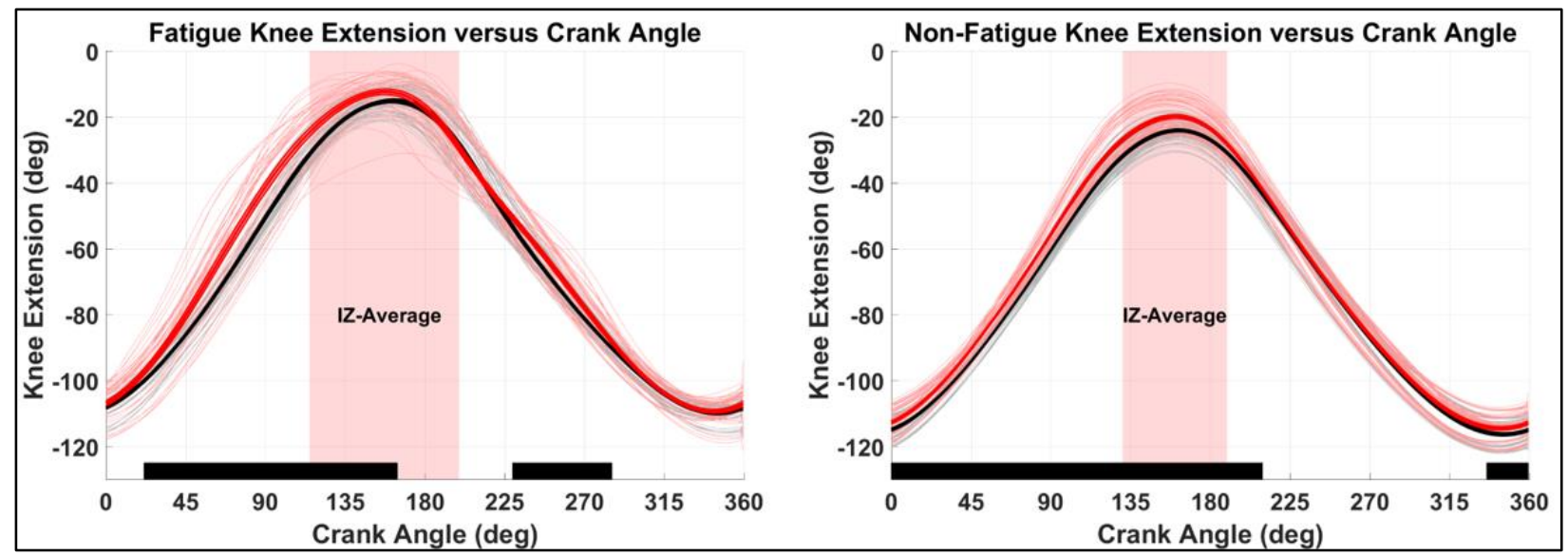

Figure 2-22: Knee Extension Over Cycle During Iso-effort Region of Endurance Ride for Fatigue (left) and Non-Fatigue (right) Groups. Initial (black) and final (red) sections of the ride section are shown. X-axis black bars indicate regions of statistically significant change in hip adduction between pre-fatigue and fatigued states $(\mathrm{p}<0.01)$.

Knee extension is considered a significant metric for ITBS injury risk exposure, as implemented in the "impingement zone" (IZ, knee extension $>-30^{\circ}$ ) of knee ROM (Farrell, Reisinger, and Tillman 2003), where the ITB is said to be inducing shear friction loads on the lateral knee. Using the current definition of IZ indicated that the Fatigue group may experience significantly greater duration of LFE contact $(\mathrm{p}<0.01)$ than Non-Fatigue subjects.

Both groups show IZ approximately centered around $155^{\circ}$ (late downstroke), which corresponds to peak knee extension at each section of the ride. Fatigue subjects consistently exhibit a lowvariability knee extension profile ranging from $-110^{\circ}$ extension (at pre-upstroke) to an average peak of $-15^{\circ}$ extension (at $155^{\circ}$ ). As the subjects fatigued, significant increases in knee extension $(\mathrm{p}<0.01)$ are seen through much of the downstroke $\left(25^{\circ}-165^{\circ}\right)$ and mid-upstroke $\left(230^{\circ}-285^{\circ}\right)$, increasing peak extension to $-10^{\circ}$ without a timing shift. Non-Fatigue subjects started the ride section with more flexed knee profile, ranging from $-115^{\circ}$ to $-25^{\circ}$ knee extension. With continued cycling, Non-Fatigue subjects also exhibited significant increases in knee extension during a larger duration of the cycle, from pre-downstroke through to early-upstroke of the next cycle $\left(340^{\circ}\right.$ $210^{\circ}$ ). Final knee extension range for the Non-Fatigue group is from $-113^{\circ}$ to $-20^{\circ}$, maintaining a significantly more flexed knee than the final kinematic profile of the Fatigue group. 

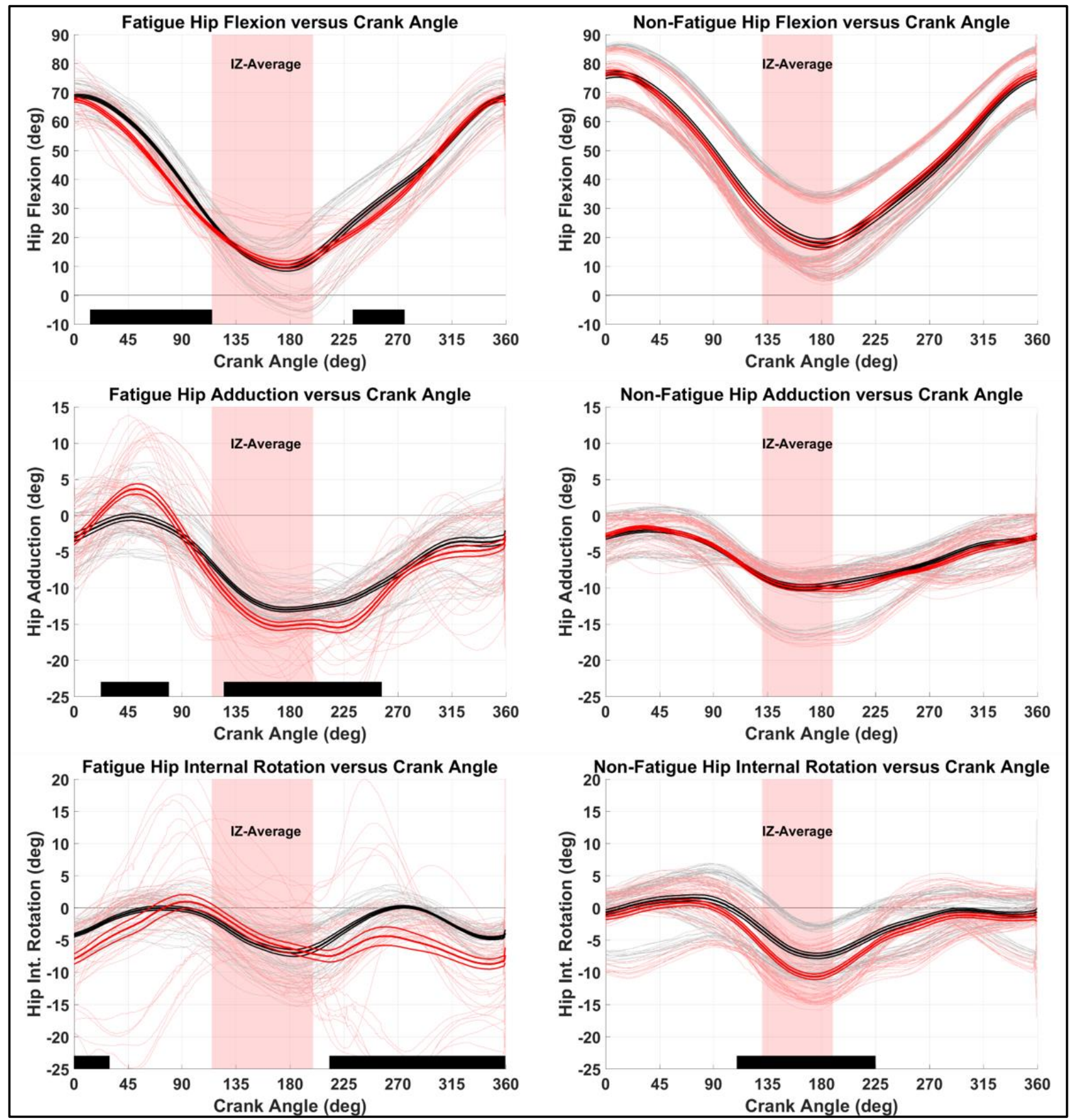

Figure 2-23: Raw and Average Hip Kinematic Angles Over Cycle in Pre-Fatigue (black) and Fatigue (red) States $(n=4) . X$-axis black bars indicate regions of statistically significant change in hip adduction between pre-fatigue and fatigued states $(\mathrm{p}<0.01)$.

Moving up the kinetic chain, three hip DOF (flexion, adduction, and rotation) were tracked with performance. Fatigue group hip flexion (range: $10^{\circ}-70^{\circ}$ ) was found to be significantly less over the crank cycle than the Non-Fatigue hip flexion profile (range: $18^{\circ}-76^{\circ}$ ). Peak hip extension occurs within the average $\mathrm{IZ}$ at $180^{\circ}$ in both groups and through the ride section. With performance 
reduction in the Fatigue group, hip flexion significantly decreased $(\mathrm{p}<0.01)$ during early-mid downstroke $\left(15^{\circ}-115^{\circ}\right)$ and mid-upstroke $\left(230^{\circ}-275^{\circ}\right)$. No significant change in hip flexion is observed in the Non-Fatigue group with continued cycling.

Initial Fatigue group hip adduction profiles show no adduction (range: $-13^{\circ}-0^{\circ}$ ), with peak adduction occurring at $45^{\circ}$ (early downstroke) and maximal abduction maintained from late downstroke to early-upstroke $\left(155^{\circ}-225^{\circ}\right)$. Fatigued behavior has a significant spike in hip adduction, averaging $+5^{\circ}$ hip adduction during early downstroke $\left(55^{\circ}\right)$. From late downstroke through early upstroke $\left(100^{\circ}-260^{\circ}\right)$, fatigued subjects exhibited statistically significant increases in bilateral hip abduction $(\mathrm{p}<0.01)$ of at most $+5^{\circ}$ abduction. Non-Fatigue subjects had less hip adduction ROM (range: $-10^{\circ}$ to $-2^{\circ}$ ), with timing and duration of peaks and maintained kinematics similar to the Fatigue group. No significant changes in Non-Fatigue hip adduction kinematics were observed with continued cycling. Adduction variance in at the end of the ride was significantly greater in the Fatigue group than the Non-Fatigue group.

Hip rotation was seen to vary for both performance groups over the ride section. Initial Fatigue hip internal rotation (range: $-7^{\circ}-0^{\circ}$ ) exhibited higher oscillation frequency than initial Non-Fatigue kinematics (range: $-7^{\circ}-1^{\circ}$ ). Peak hip internal rotation in both groups occur at mid-downstroke $\left(\sim 70^{\circ}\right)$ and mid-upstroke $\left(\sim 270^{\circ}\right)$, with peak external rotation at the bottom of the cycle $\left(180^{\circ}\right)$. Following performance decline, Fatigue subjects showed an insignificant increase in hip internal rotation $\left(+1^{\circ}\right)$ during late downstroke, while a significant increase in external rotation $\left(+5^{\circ}\right.$, $\mathrm{p}<0.01)$ was observed from early upstroke to mid-downstroke $\left(200^{\circ}-75^{\circ}\right)$. Timing of internal/external rotation peaks were also affected, resulting in delayed peak downstroke internal rotation (moving from $70^{\circ}$ to $90^{\circ}$ ) and spatially faster internal rotation during early upstroke. NonFatigue hip external rotation significantly increased $\left(+5^{\circ}, \mathrm{p}<0.01\right)$ from late downstroke through early upstroke $\left(105^{\circ}-225^{\circ}\right)$. A uniform, but insignificant, increase in external rotation is also seen over the remaining cycle $\left(225^{\circ}-105^{\circ}\right)$. No changes in hip rotation timing were observed across the ride sections for Non-Fatigue subjects. 
At the upper boundary condition of the leg, the pelvis and torso center-of-mass (COM) were tracked. COM translation was quantified as deviation from the average initial COM position, and is presented below [Figure 2-24 and Figure 2-25].
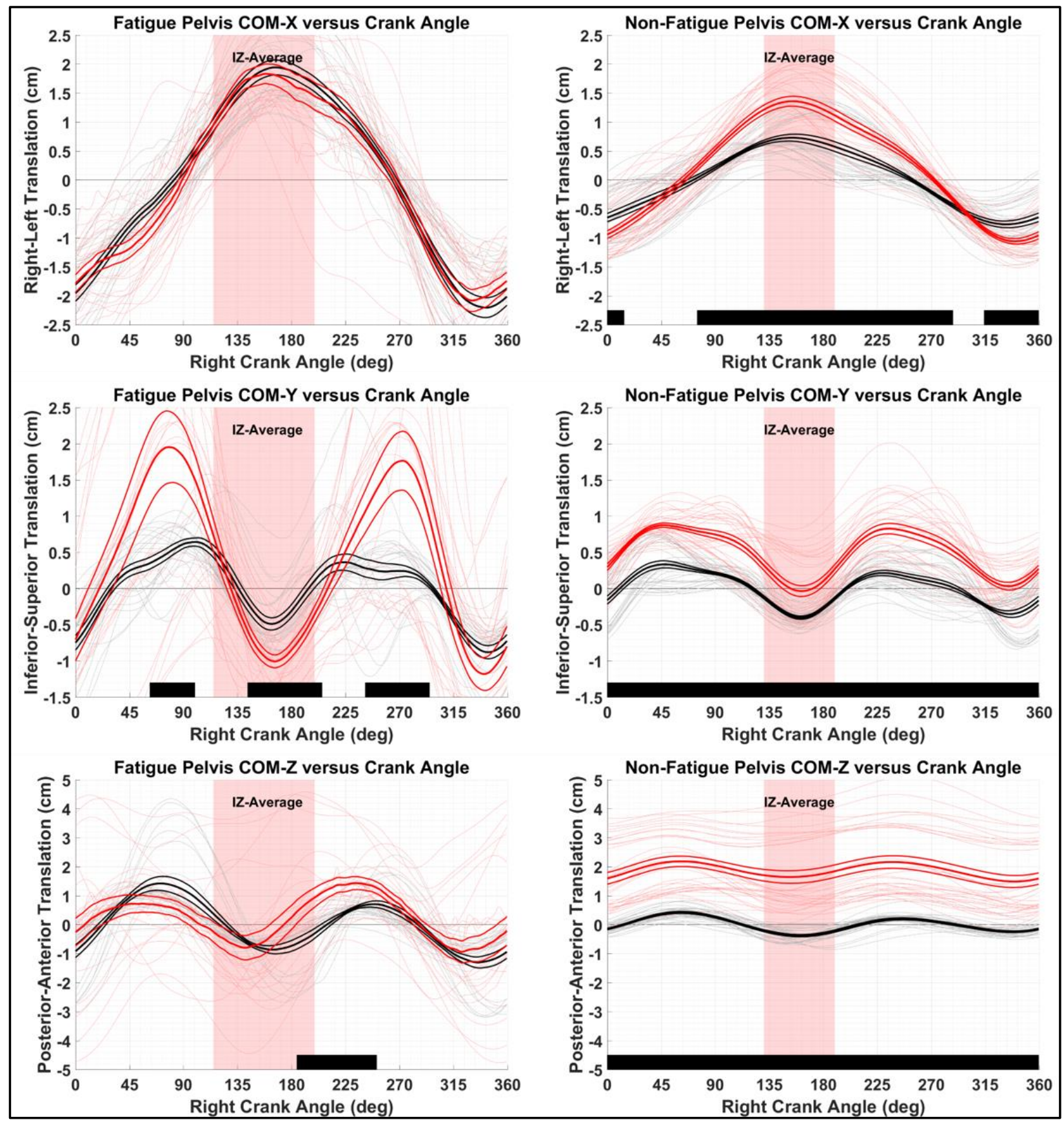

Figure 2-24: Pelvis COM Translation Over Cycle During Endurance Ride. Initial (black) and final (red) sections for Fatigue (left) and Non-Fatigue (right) groups are shown. X-axis black bars indicate regions of statistically significant change between initial and final states $(p<0.01)$. 
Initial pelvis COM ROM for the Fatigue subjects was significantly greater and more variable than that of Non-Fatigue subjects, while the timing of peak COM motion was consistent between groups. For both groups, lateral translation peaks toward the side of the next downstroke approximately $30^{\circ}$ prior to the downstroke. Pelvis COM translates upward during early downstroke, maintaining a superior position through mid-downstroke. This was followed by quick inferior translation through mid-to-late downstroke $\left(120^{\circ}-160^{\circ}\right)$ before returning superiorly for the contralateral downstroke. Posterior-anterior translation of the pelvis COM initially follows a similar trend, translating forward before and during downstroke, and retuning posteriorly at the end of the downstroke.

Following endurance cycling, both groups exhibited significant adjustments to pelvis COM motion. Fatigue subjects did not significantly change lateral pelvis motion with fatigue, but showed significantly increased vertical translation inferiorly and superiorly $(\mathrm{p}<0.01)$. Timing in the cycle did not change, for vertical translation, but the previous superior COM position through downstroke became dynamic throughout the cycle. Anterior-posterior motion showed a significant anterior translation during early left-leg downstroke $\left(185^{\circ}-250^{\circ}\right)$. A timing shift is observed such that peak anterior translation precedes peak superior translation, and peak posterior translation precedes peak inferior translation.

Non-Fatigue subjects show significantly increased lateral sway $(+1 \mathrm{~cm} R O M, p<0.01)$ a superior translation of pelvis COM, but with a significant increase in lateral sway. Significant superior $(0.5 \mathrm{~cm})$ and anterior $(2 \mathrm{~cm})$ translation of the pelvis COM $(\mathrm{p}<0.01)$ is observed uniformly across the cycle. No significant shifts in pelvis COM translation timing were observed for the NonFatigue subject group. 

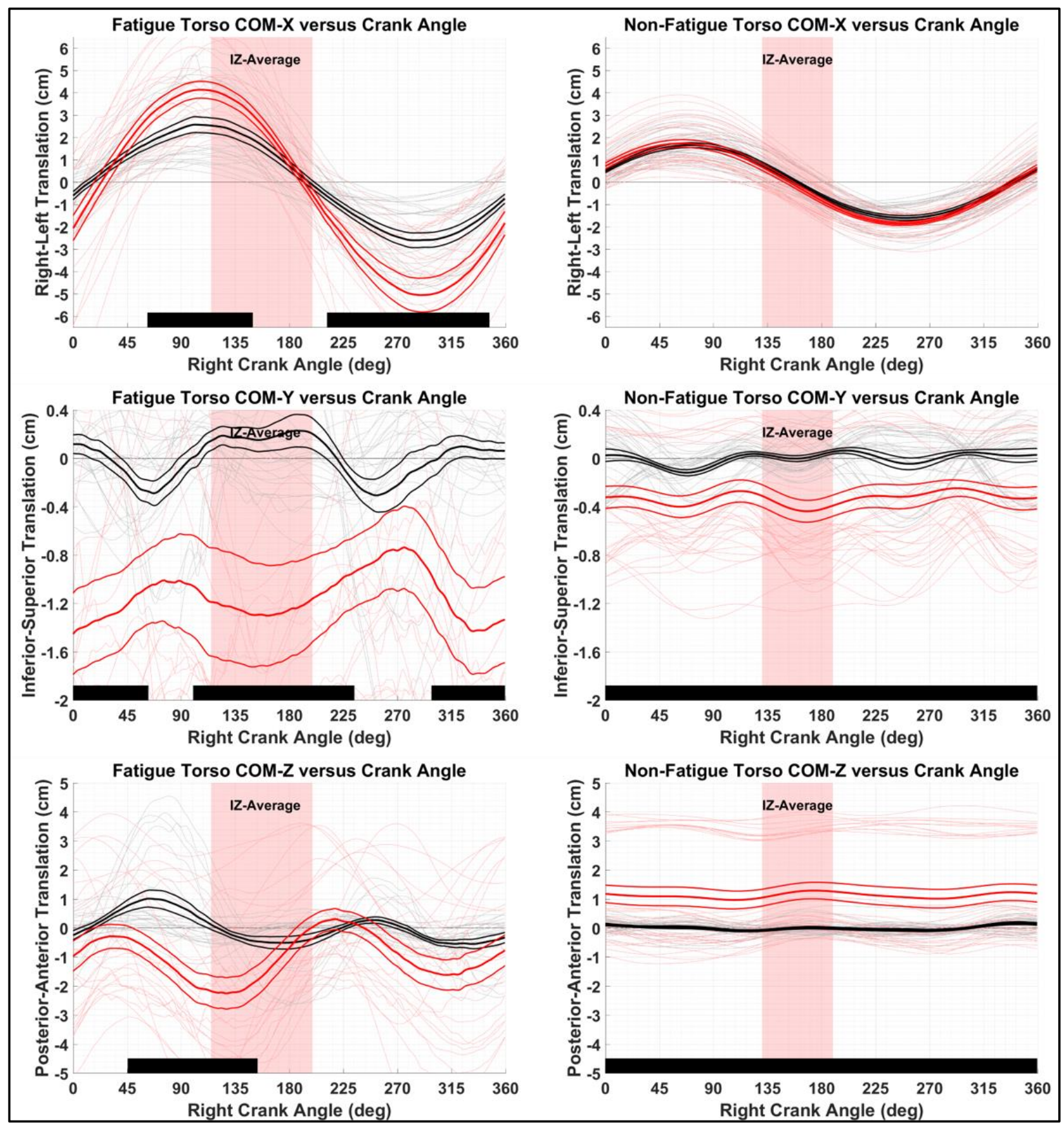

Figure 2-25: Torso COM Translation Over Cycle During Endurance Ride. Initial (black) and final (red) sections for Fatigue (left) and Non-Fatigue (right) groups are shown. X-axis black bars indicate regions of statistically significant change between initial and final states $(\mathrm{p}<0.01)$.

Initial Fatigue group torso ROM [Lat: $\pm 2.5 \mathrm{~cm}$, Sup: $\pm 0.25 \mathrm{~cm}$, Ant: $\pm 1 \mathrm{~cm}$ ] was significantly larger than Non-Fatigue group torso ROM [Lat: $\pm 1.8 \mathrm{~cm}$, Sup: $\pm 0.1 \mathrm{~cm}$, Sup: $\pm 0.1 \mathrm{~cm}$ ]. Torso and pelvic COM translation track similarly over initial cycles for Fatigue subjects. Lateral translation of the torso preceded pelvis translation in both performance groups across cycle sections, peaking 
towards the contralateral side during mid downstroke. Fatigue subjects exhibited a significant increase in lateral sway following performance reduction $(+4 \mathrm{~cm} R O M, p<0.01)$, with preferentially increased lateral motion toward the right. Inferior-superior torso COM translation initially peaked superiorly during lowest pelvis COM translation, and vice versa. Following fatigue, torso COM displayed significant inferior shift during pre-to-early downstroke bilaterally $(-1.4 \mathrm{~cm}, \mathrm{p}<0.01)$ and shifted in time to varied along with pelvis vertical translation. A significant posterior translation of the torso is observed during mid-to-late right-leg downstroke $\left(45^{\circ}-150^{\circ}\right)$. Mean posterior shift on the left leg downstroke is also present, but not significant.

Non-fatigue torso ROM shows no significant change in lateral motion over the cycle. Torso COM uniformly shifts inferiorly by $-0.3 \mathrm{~cm}$, which was significant $(\mathrm{p}<0.01)$, but may not be clinically significant. Significant anterior translation of the torso COM is observed uniformly across the cycle $(+1 \mathrm{~cm}, \mathrm{p}<0.01)$.
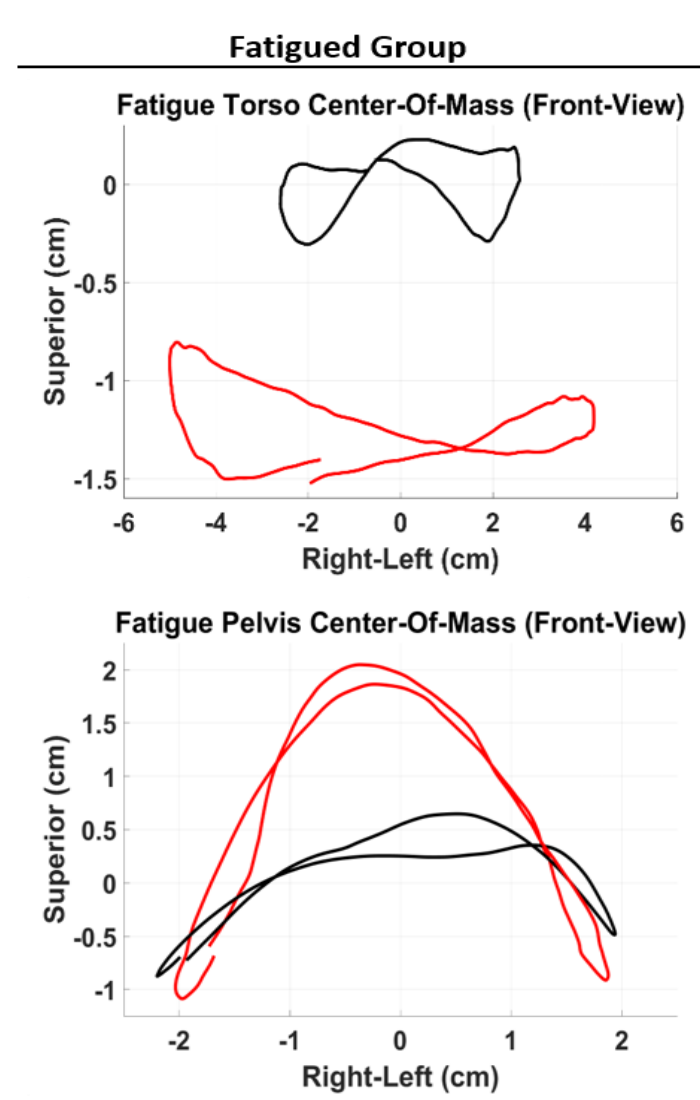

Non-Fatigued Group

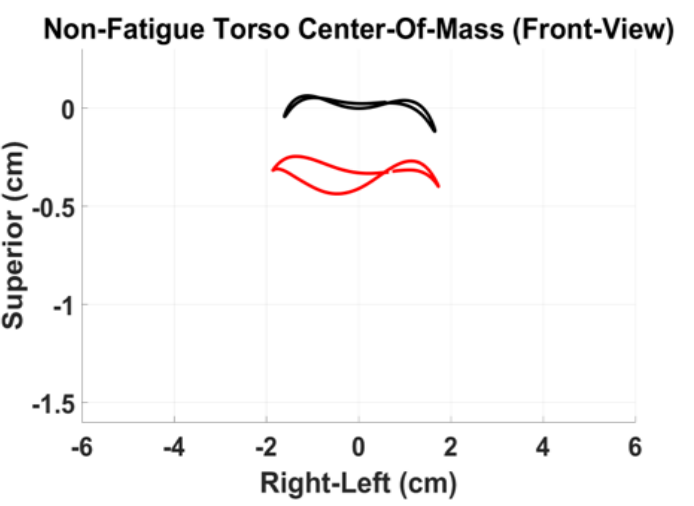

Non-Fatigue Pelvis Center-Of-Mass (Front-View)

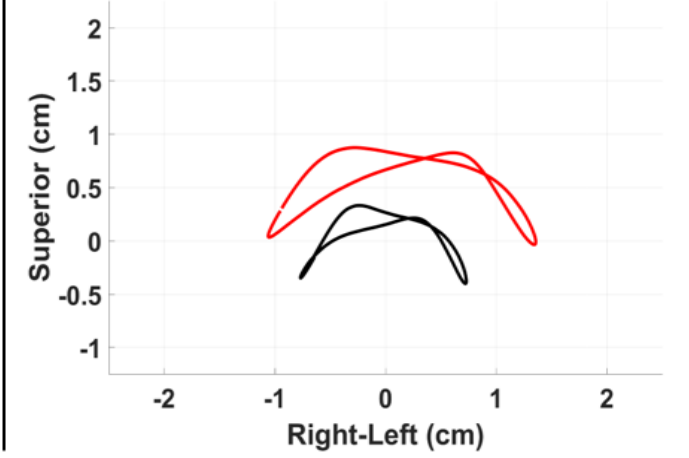

Figure 2-26: Coronal-view of Average Torso COM (top) and Pelvis COM (bottom) Trajectories Over Cycle During Isokinetic Region of Endurance Ride for Fatigue (left) and Non-Fatigue (right) Groups. Initial (black) and final (red) sections of the isokinetic region ride are shown. 
Combining two of the three coordinates enables spatial visualization of the trajectory and comparison of pelvic and torso COM motions between groups and across ride sections. Presentation of the frontal plane motion is provided in Figure 2-26, above.

All torso COM trajectories were primarily horizontal, with coupled lateral-vertical behavior observed through the inferior-superior translation at lateral extremes of motion. Similar dynamics are observed at the pelvis, but of significantly larger magnitude in the fatigued state. Frontal plane COM trajectories show "figure eight" motion as the subjects shift torso and pelvis mass.
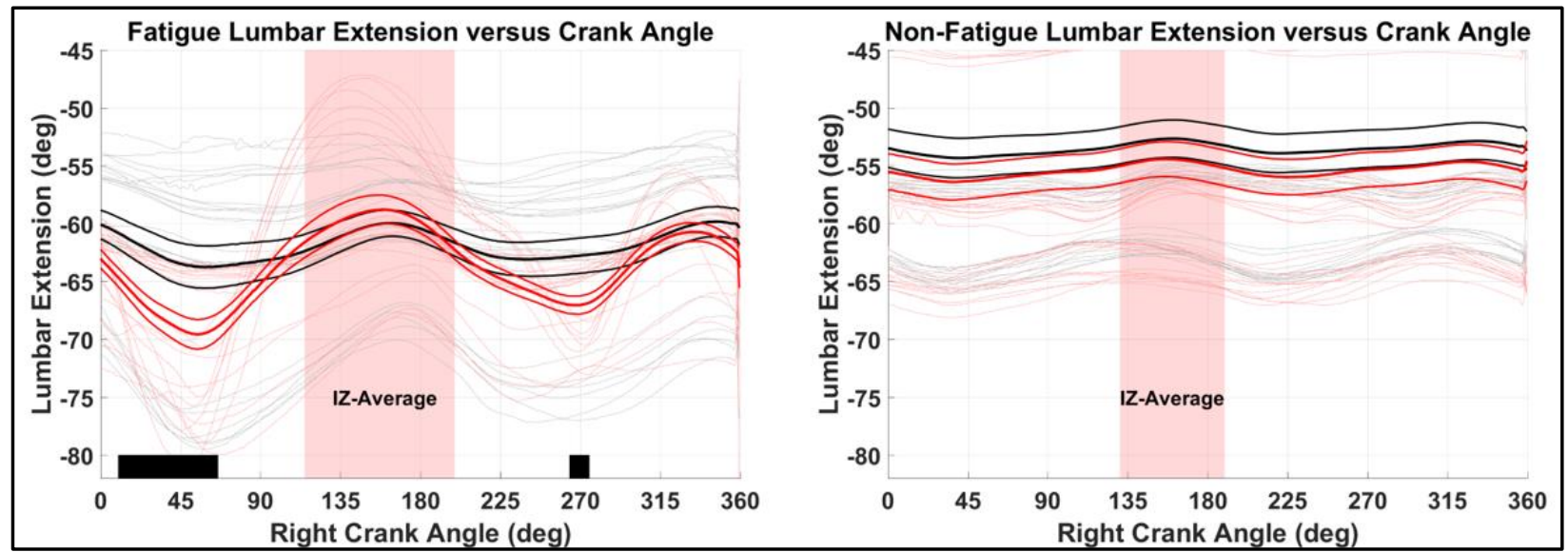

Figure 2-27: Lumbar Extension Over Cycle During Iso-effort Region of Endurance Ride for Fatigue (left) and Non-Fatigue (right) Groups. Initial (black) and final (red) sections of the ride section are shown. X-axis black bars indicate regions of statistically significant change in hip adduction between pre-fatigue and fatigued states $(\mathrm{p}<0.01)$.

The osteokinematic expression of relative torso-pelvis motion is lumbar extension or flexion. Initial average lumbar extension for the Fatigue group ranged from $-63^{\circ}$ extension to $-60^{\circ}$ extension, with peak flexion during mid-downstroke $\left(45^{\circ}-120^{\circ}\right)$ and mid-upstroke $\left(225^{\circ}-290^{\circ}\right)$. With fatigue onset, significant increases in peak lumbar flexion were observed $(\mathrm{p}<0.01)$ during similar peak flexion regions $\left(5^{\circ}-80^{\circ}\right.$ and $\left.260^{\circ}-275^{\circ}\right)$. Maximum lumbar extension increased insignificantly during late downstroke $\left(\sim 160^{\circ}\right)$. Initial Non-Fatigue lumbar extension (range: $-54^{\circ}$ to $-53^{\circ}$ ) was significantly greater than that of Fatigue subjects (range: $-63^{\circ}$ to $-60^{\circ}$ ). Slight oscillations in lumbar flexion are observed during mid-downstroke and mid-upstroke. At the end of the ride section, Non-Fatigue subjects exhibited a uniform, but insignificant, increase in lumbar flexion over the cycle. 


\subsubsection{PHYSICAL CHARACTERISTICS}

A preliminary comparison of non-fatigue and fatigue sub-groups was conducted. No strong performance correlations with training regularity and competition style were found, but additional studies with larger sample sizes can help find physical and behavioral metrics correlated with activity performance. To test for differences in physical characteristics between the fatigue categories, a series of t-tests were run. Results from these tests are presented below [Table 2-8].

Table 2-8: Student's t-Test Results for Volunteer Parameters versus Performance Category

\begin{tabular}{|c|c|c|c|c|}
\hline \multirow[b]{2}{*}{ Parameter } & \multicolumn{2}{|c|}{ Mean Value (Standard Error) } & \multirow[b]{2}{*}{$\begin{array}{c}\text { Mean } \\
\text { Difference }\end{array}$} & \multirow[b]{2}{*}{$\begin{array}{c}\text { Two-tailed } \\
\text { p-Value }\end{array}$} \\
\hline & $\begin{array}{c}\text { Non-Fatigued } \\
(n=4)\end{array}$ & $\begin{array}{c}\text { Fatigued/Failed } \\
(n=5)\end{array}$ & & \\
\hline $\operatorname{Sex}(\mathrm{M} / \mathrm{F})$ & $4 \mathrm{M} / 0 \mathrm{~F}$ & $5 \mathrm{M} / 0 \mathrm{~F}$ & --- & --- \\
\hline Height (in.) & $70.75(3.20)$ & $72.4(2.07)$ & -1.65 & 0.207 \\
\hline Weight (lbs.) & $177.5(10.41)$ & $172.4(15.66)$ & 5.10 & 0.299 \\
\hline $\mathrm{BMI}$ & $24.96(1.23)$ & $23.10(1.62)$ & 1.85 & $0.047^{\dagger \dagger}$ \\
\hline Age (yrs.) & $33.5(9.33)$ & $30.8(9.68)$ & 2.70 & 0.343 \\
\hline Hip-to-Heel Length (in.) & $42.25(1.54)$ & $44.3(1.93)$ & -2.05 & $0.060^{\dagger}$ \\
\hline Hip-to-Head Length (in.) & $28.5(1.85)$ & $28.1(1.53)$ & 0.40 & 0.371 \\
\hline Leg Length Ratio & $0.60(0.01)$ & $0.61(0.02)$ & -0.01 & $0.096^{\dagger}$ \\
\hline
\end{tabular}

${ }^{\dagger} \mathrm{p}$-value significant at $\alpha=0.1,{ }^{\dagger \dagger} \mathrm{p}$-value significant at $\alpha=0.05$

While baseline anthropometry is statistically similar between the performance groups, composite metrics show significant differences. BMI ( $\mathrm{p}<0.05)$, Hip-to-Heel Length $(\mathrm{p}<0.1)$, and Leg Length Ratio ( $\mathrm{p}<0.1)$ differences indicate influence of higher BMI with shorter legs in cycling fatigue. Volunteer height, weight, and age did not significantly vary between Fatigue and Non-Fatigue performance groups.

\subsubsection{PERFORMANCE SUMMARY}

To visualize performance metric changes, the following plots [Figure 2-28] were developed to present the timing and direction of statistically significant variations between the start and end of the analyzed cycle sections. The impingement zone and the level of significance can be shown by color coding of the bars, as well. 

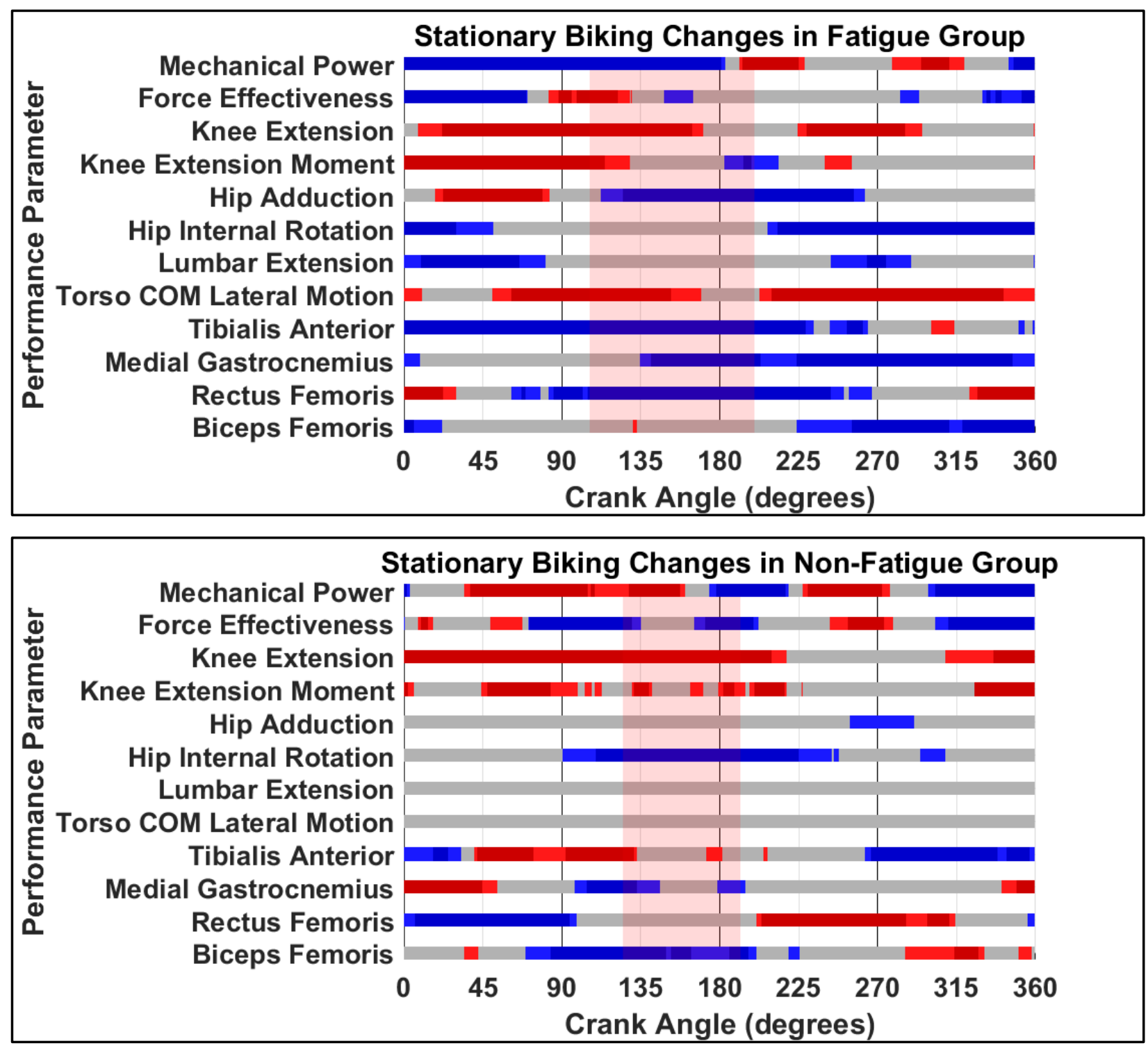

Figure 2-28: Summary of Statistically Significant Changes within Fatigue and Non-Fatigue Groups. Significant $(\mathrm{p}<0.05)$ increases and decreases are indicated by red and blue, respectively. Strongly significant $(\mathrm{p}<0.01)$ changes are indicated by darkened blue and red regions.

Both performance groups exhibited significant changes $(\mathrm{p}<0.05$ and $\mathrm{p}<0.01)$ over their endurance ride sections. While kinematic changes were observed, the magnitude of change was larger in the Fatigue group. Upper body kinematic changes were less dynamic among Non-Fatigue subjects, leading to broad, but relatively stable shifts in osteokinematics. EMG signals in Fatigue subjects significantly decreased over the ride section, distal muscle groups with minimal activity. Compensatory activation of proximal muscle groups correlated with broader hip adduction, hip rotation, and torso ROM following fatigue onset. 


\subsection{DISCUSSION AND CONCLUSIONS}

\subsubsection{FATIGUe GROUP PERFORMANCE TRENDS}

\section{Kinetics vs. Performance}

Mechanical power shows peak magnitude during downstroke, and low-magnitude negative power during upstroke, which agrees with literature trends (Sanderson 1991). Significant fatigue-induced changes over the cycle are observed with reduced power generation during down stroke and periods of increased (less negative) power output in up stroke. Similar decreases in power with fatigue are seen across activities, such as walking (Enoka and Duchateau 2016). However, it should be noted that the up stroke unilateral mechanical power output is low and hence has a lower impact on net mechanical power over the cycle.

Force application effectiveness is observed to significantly decrease in pre-upstroke and predownstroke portions of the cycle in both performance groups. Efficacy reduction is also seen in early downstroke in the Fatigue group, while reduction is seen in mid-to-late downstroke in the Non-Fatigue group. Fatigue group shows statistically significant effectiveness increases during peak power generation.

\section{Kinematics vs. Performance}

During $0^{\circ}$-to- $90^{\circ}$ down stroke, the Fatigue group exhibited a net $\sim 5^{\circ}$ hip adduction coinciding with fatigue and the onset of performance failure, while pre-failure kinematics exhibit no significant adduction as seen in literature (Rodrigo R. Bini et al. 2016). Adduction peaks, when the pelvis is shifted to a lateral position toward the downstroke leg, would explain bilateral knee instability that was qualitatively observed. Fatigue-induced hip adduction is concurrent with increased hip external rotation, lumbar flexion, and torso COM lateral excursion. Late down stroke kinematic changes differ from the early down stroke trends with significantly increased hip abduction and increased hip internal rotation. Both factors are found to be characteristic of symptomatic ITBSafflicted athletes in running (Aderem and Louw 2015). Up stroke following performance reduction exhibits increased hip abduction, hip external rotation, lumbar flexion, and upward (superior) 
translation of pelvis COM. Transition of power generation toward inertial compensation may account for superior translation of pelvis COM and lateral knee swing, likely accompanied by increased hip hike as weight is shifted to the contralateral active limb. Over the entire cycle, fatigued torso COM exhibits lowered (inferior) positioning relative to pre-fatigue regimes of the test measurements.

Increased upper body sway tended to coincide with the onset of performance reduction/failure. Observed inferior shifts of the torso COM suggests increased lumbar flexion with continued cycling and may be implicated in the development of NS-LBP (Van Hoof et al. 2012). Pelvis and torso COM trajectories showed significant changes in frontal plane across both performance groups, often not only effecting lateral motion, but inducing a rocking vertical translation as weight is shifted from one side of the bike to the other.

Changes in Fatigue group ankle kinematics directly correspond to changes in pedal kinematics, decreasing overall IE (Lanferdini et al. 2016). Much of the force efficacy change can be attributed to distal muscle weakening. In the Non-Fatigue group, ankle kinematics are adjusted with minimal changes in the pedal orientation, indicating effective compensation of ankle/knee/hip kinematics and muscle activation patterns.

\section{EMG vs. Performance}

Initial activation patterns observed in both performance groups were in line with literature on/off ranges (Jorge and Hull 1986). Lower extremity muscle activation profiles show distinct changes with the onset of significant performance reduction. Tibialis Anterior and Medial Gastrocnemius signal intensity decreases relative to pre-fatigue levels, indicating loss of foot fine motor control. Rectus Femoris and Biceps Femoris, considered large cycling force generation muscles, exhibit approximately $-90^{\circ}$-shifted activation profiles. Similar adaptations are observed in literature with fatigue onset (So, $\mathrm{Ng}$, and $\mathrm{Ng}$ 2005; Hautier et al. 2000). Interpreted as compensation for failure to pre-align the foot following $\mathrm{TA} / \mathrm{MG}$ weakness, the $\mathrm{RF} / \mathrm{BF}$ activation phase-shift also accompanies shorter signal pulse durations without significant decreases in peak $\mathrm{RF} / \mathrm{BF}$ signal. The observed coactivation of the hamstring muscles to compensate for low cadence performance has been reported to relieve foot eversion stresses at high resistance levels (So, $\mathrm{Ng}$, and $\mathrm{Ng} \mathrm{2005).}$ 


\subsubsection{FATIGUE GROUP INTER-VARIATE TRENDS}

During the power-generating down stroke of the lower extremity $\left(350^{\circ}-180^{\circ}\right)$, significant performance reduction accompanied a variety of changes: foot fine motor control loss, phaseshifted force-generation muscle activation, increased hip adduction, increased hip internal rotation, increased lumbar flexion, decreased average power efficiency, and greater torso COM lateral excursion. Although current steps are being taken to increase resolution over the isokinetic performance loss period, it appears that fatigue of fine motor control muscles may have led to earlier, less efficient RF/BF activation, and subsequent kinematic changes. Power efficiency shows slight increases in late down stroke, which could be explained by lateral torso sway, shifting torso $\mathrm{COM}$ over the pedal and thereby relying on inertial force-generation during BF activation and subsequent knee flexion. Hip adduction peak may be induced by hip abductor weakness and future analysis will target this failure mechanism and its implications in IT Band Syndrome.

Significant loss of upstroke muscle activation aligns with decreased power efficiency, increased hip abduction, increased hip external rotation, and reduced lumbar flexion duration. Although up stroke power generation is lost and lowered net efficiency, up stroke power deficit resulting from a "dragged" contralateral limb is reduced. Diminished dragging behavior resulted in a more powerneutral up stroke regime, where the contralateral limb no longer significantly hindered power generation in the active down stroke limb.

\subsubsection{Non-FAtigue vs. FATIGUe COMPARISON}

\section{Volunteer Characteristic Differences}

Statistical testing of the volunteer physical characteristics indicates that there are no statistical differences in height, weight, or age associated with the level of performance in this study. However, BMI $(\mathrm{p}<0.05)$ and hip-to-heel length $(\mathrm{p}<0.1)$ were found to have statistically significant differences between the "Fatigue" and "Non-Fatigue" groups. Participants exhibiting fatigue resistance tended to have a higher BMI, and a shorter hip-to-heel length. Normalizing hipto-heel length by height ("Leg Length Ratio") yielded a slightly weaker difference between performance categories, and while still statistically significant $(\mathrm{p}<0.1)$, the finding may not be clinically significant (1\% difference between groups). 
While BMI does not distinguish fat and muscle weight, the significantly greater BMI among the "Non-Fatigue" individuals could be influenced by greater muscle mass is correlated with greater increased ability to effectively deliver power in relatively short duration, high resistance cycling (Craig and Norton 2001). Measurement of body fat percentage in future studies would remove BMI's confounding fat-vs-muscle variable. Adding hip-to-heel length trends, the effect of BMI and leg length could have two explanations: 1) the greater muscle mass per unit length of leg provides greater energy density for "Non-Fatigue" volunteers, or 2) the longer legs of "Fatigue" volunteers results in a larger moment arm for bike resistance, putting a greater load on distal foot control muscles per unit of resistance torque.

\section{Volunteer Performance Differences}

Kinematics While both performance groups exhibited decreased knee extension with continued cycling $(\mathrm{p}<0.05)$, the Fatigue group tended to be more extended throughout the crank cycle. Fatigue group also spent a greater duration of the cycle in the lateral femoral epicondyle "Impingement Zone" (knee flexion $<30^{\circ}$ ). Initial hip abduction was greater in the Fatigue group between late downstroke and mid-upstroke. As this group decreased RPM, significant knee adduction $\left(+5^{\circ}\right)$ was observed prior to mid-downstroke power generation. The Non-Fatigue group did not exhibit net hip adduction or significant changes during the selected ride section. Regarding hip rotation, initial mean hip internal rotation was found to be greater for Non-Fatigue subjects, corresponding with similar findings by Bini et al. for competitive cyclists versus recreational cyclists (Rodrigo R. Bini et al. 2016). Both groups showed increased external hip rotation, but the duration and phase of this change were unique to each group. The Fatigue group tended to externally rotate the hip from mid-upstroke to early downstroke, while the Non-Fatigue group consistently exhibited a change of similar magnitude during a shorter region of the cycle, from late-downstroke to early-upstroke. Non-Fatigue volunteers were significantly more lumbar flexed throughout the isokinetic ride section analyzed. In addition, this group showed a uniform, but not statistically significant ( $>00.05)$, increase in flexion with continued cycling. Fatigued subjects, while more flexed throughout, exhibited significant increases $(\mathrm{p}<0.05)$ in lumbar flexion with corresponding to power generation strokes. Fatigue-induced increases in lumbar flexion tended to 
shortly precede significantly increased lateral excursion of the torso center-of-mass. Center-ofmass excursion among Non-Fatigue individuals showed no significant change.

Kinetics Power output and force efficacy exhibited changes with continued cycling. As expected during isokinetic RPM changes, peak downstroke mechanical power decreased for the Fatigue group, while it increased in the Non-Fatigue group $(\mathrm{p}>0.05)$. While Non-Fatigue subjects significantly increased mechanical power during downstroke, it is important to note that this is confounded with the increase in resistance. Resistance was increased manually to maintain selfreported effort level, and limits conclusions that are made from uncorrected (unnormalized/weighted) kinetic measures.

Downstroke force efficacy during peak mechanical power, however showed an inverse relationship with performance group. While early-downstroke force efficacy decreased in the Fatigue group, a net increase in efficacy is seen during mid-to-late downstroke, indicating potential kinematic compensation. Peak power increases in the Non-Fatigue subjects corresponded with decreased downstroke efficacy. The Non-Fatigue group shows effectiveness increases only before and after peak mechanical power generation. While effectiveness appears to improve prior to upstroke, power generation during does not seem to significantly improve. Maintained mechanical power with significantly altered kinematics hints toward the development of successful compensation mechanisms in the Non-Fatigue performance group. The influence of changes in upstroke performance corresponded with subject performance, and may assess when system level performance will decrease, or resilience to long-duration tasks.

EMG Trends Comparing EMG signal morphology changes between the performance groups indicates two significant differences in the distal and proximal muscle behaviors. Fatigue group distal muscles (TA/MG) showed significantly decreased signal amplitude, with no significant shift in activation timing. Proximal power generation muscles in the Fatigue group, however did not lower activation strength, but activated significantly earlier in the crank cycle: BF activation moving from mid-upstroke to late downstroke, and RF moving from mid-downstroke to pre-toearly downstroke. In fact, fatigued-state BF and RF timing approximately corresponded with the 
pre-fatigue MG and TA peak activation timing, respectively. Non-Fatigue group EMG profiles did not show significant changes in peak activation signal, but the timing of all muscles tended to shift earlier in the crank cycle with continued cycling. Similar to the inter-muscle timing of the Fatigue group, the Non-Fatigue group's RF and BF activation were shortly preceded by TA and MG activation, respectively. While performance was maintained in this group, the relative timing of these muscles was also maintained, even with a timing shift. From these observations, a notion of muscle activation "flexibility" can be defined, relating to ability of the activation profile to change to counteract the effects of fatigue. Both performance groups showed "flexible" activation of the proximal power generation muscles. Differences emerge in the distal muscles, where the Fatigue group had limited flexibility in TA/MG activation. Inability of the fatigued volunteers to significantly adjust distal muscle timing along with RF/BF changes may lead to increased loading, and early fatigue, of foot dorsi-/plantar-flexion muscles. 


\section{Chapter 3}

EMG-Based Regression

Analysis

\subsection{INTRODUCTION}

Current human fatigue or performance models draw from medical records, sleep performance, and comprehensive activity tracking (R. Bini, Hume, and Croft 2011; van den Bogert et al. 2013; Cecchini et al. 2014). While these models have predictive strength, they are limited in their generalizability to other activities and to the wider population (Noakes 2000). Lower level hierarchical models and neural networks are also investigated to predict muscle dynamics, but would require higher computational cost that could limit extension to real-time applications (Cecchini et al. 2014; Heidlauf et al. 2016). Through this investigation, the model development approach has been reevaluated.

While Chapter 2 focused on analysis of osteokinematic and EMG metrics at each section of the ride, performance modeling in this chapter aims to use simple linear regression analysis to better understand development of fatigue. Data sources considered for this study include physiological (muscle activation), kinematic, and kinetic measures from instrumented stationary biking volunteers. Given the limitations in in vivo joint instrumentation, real-world motion tracking, and loadcell implementation on a large scale, only muscle activation parameters were used (Kleissen et al. 1997).

The following sections serve to present the methods and results of the conducted EMG-based regression modeling of system-level performance. Topics will include: EMG processing, parameter definitions, regression methods, model development, and model evaluation. 


\subsection{CHAPTER OBJECTIVES}

The objectives of Chapter 3 on EMG-based regression analysis are as follows:

1) Isolate sEMG signal parameters correlated with instantaneous cycling RPM across system-level performance groups. Signal morphology metrics are defined and tracked over the endurance ride to identify parameters correlated with overall performance.

2) Develop EMG-based linear models and evaluate predictive strength. Stepwise backward elimination regression analyses are conducted to identify potential EMG metrics for future performance prediction in dynamic, minimally-instrumented applications.

3) Provide insight on generalizability of EMG-based predictive models. Predictive EMG metrics are analyzed to identify performance-critical muscle groups and trends for potential generalization to other long-duration activities.

\subsection{METHODS}

\subsubsection{MUSCLES OF INTEREST}

While testing included abdominal and back EMG, primary muscles and structures of interest were limited to the lower extremity. EMG signals from the rectus femoris (RF), biceps femoris $(\mathrm{BF})$, medial gastrocnemius (MG), and tibialis anterior (TA) were measured bilaterally [Figure 3-1]. Due to sensor malfunction, left-leg BF data was excluded from analysis across all participants. 


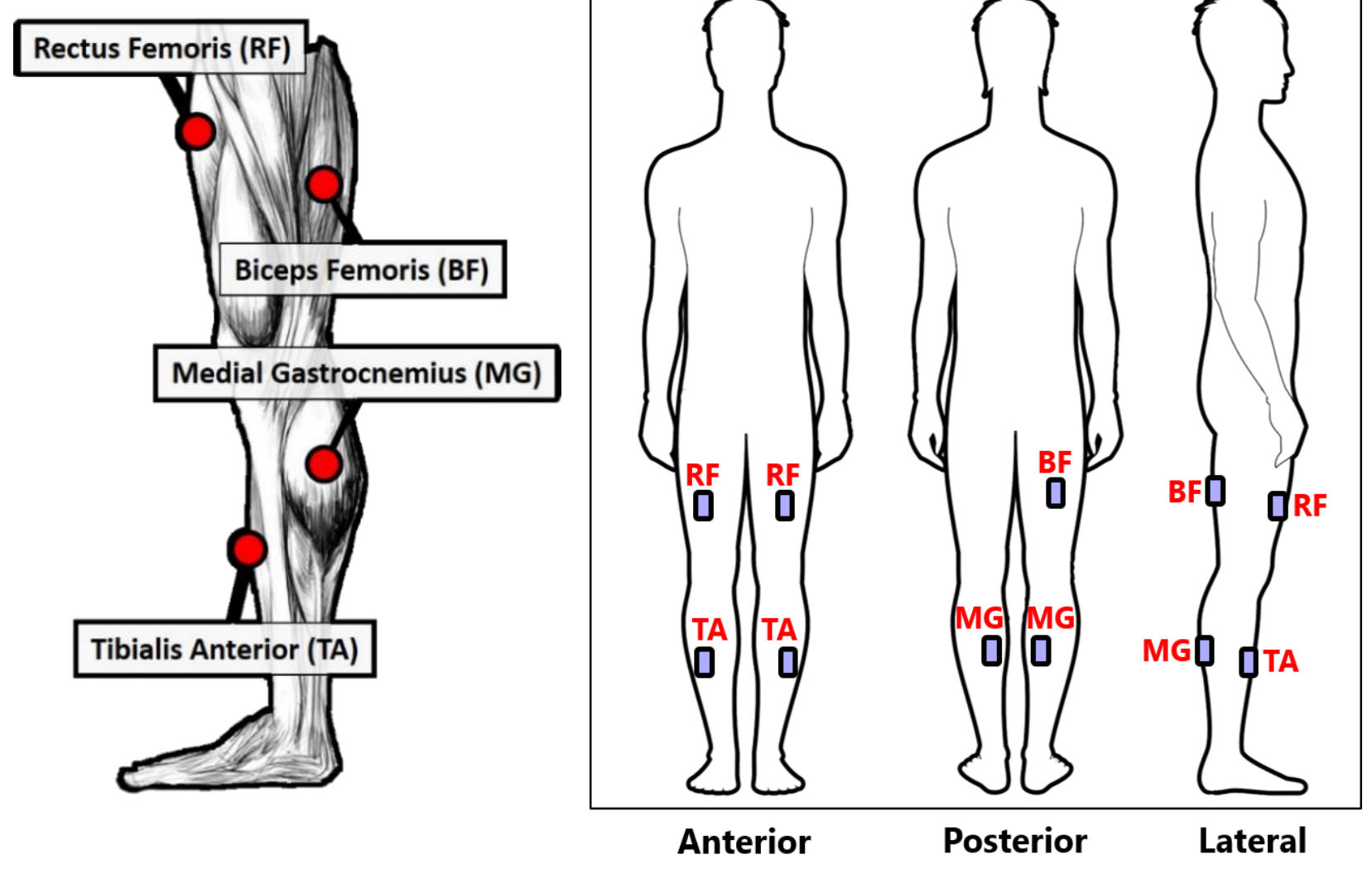

Figure 3-1: Muscles of interest for EMG Placement and Measurement

\subsubsection{EMG PRE-PROCESSING: DYNAMIC THRESHOLDING}

Standard thresholding methods for static (no body motion) EMG analysis entail finding the max value of an activation pulse, and thresholding at a fixed proportion of observed max (Jorge and Hull 1986; Kwatny, Thomas, and Kwatny 1970; Hug 2011). For cyclic EMG measurements, this method fails to account for uniform decreases in signal strength over a cycle. In the case of fatigue tracking, dynamic thresholding allows for EMG parameters to be measured, even if the max signal for fatigued cycle is significantly less than initial levels (Ozgünen, Celik, and Kurdak 2010).

For this investigation, a setpoint threshold of 0.2 of the normalized calibrated max (1a) was used. To define the "calibrated max" for a subject-muscle in a trial, the maximum signal amplitude of the first 25 cycles (1b) was averaged. The calibration average max signal (2) was used to normalize the entire EMG signal (3) for each subject-muscle. 


$$
\begin{aligned}
& \text { Tol }_{\text {setpoint }, t}=0.2 \quad \mid \quad N_{\text {calibCycles, } t}=25
\end{aligned}
$$

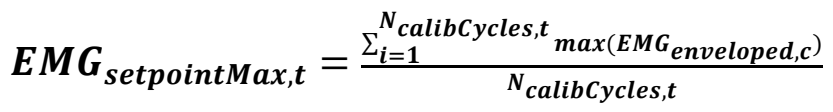

$$
\begin{aligned}
& E M G_{\text {norm }, c}=\frac{E M G_{\text {enveloped }, c}}{E M G_{\text {setpointMax }, t}}
\end{aligned}
$$

Once normalized, the signal for a cycle is thresholded using an adjusted tolerance level, defined by the product of the setpoint threshold for the trial and the normalized cycle's max (4). In this way, only EMG signals normalized to "non-fatigue" signal levels are used for analysis and parameter generation.

$$
\text { Tol }_{\text {adjusted,c }}=\text { Tol }_{\text {setpoint }, t} * \max \left(E M G_{\text {norm }, c}\right)
$$

\subsubsection{EMG PARAMETER DEFINITIONS}

For EMG analysis and performance prediction, a subset of five morphological signal parameters were defined and calculated for each cycle. The five parameters include:

- Max Activation Signal (Normalized Calibration Max)

- Average Activation Signal (Normalized to Calibration Max)

- Activation Duration (Proportion of Cycle)

- Activation Timing (Proportion into Cycle)

- Activation "Energy"

After normalization of the EMG signal cycles, the max activation signal was found as the absolute max over the cycle. For the remaining parameters, the normalized signal was first thresholded (5).

$$
\text { Active }_{c, i}=\left\{\begin{array}{l}
1 \text { for } E M G_{\text {norm }, c, i} \geq \text { Tol }_{\text {adjusted }, c} \\
0 \quad \text { else }
\end{array}\right.
$$




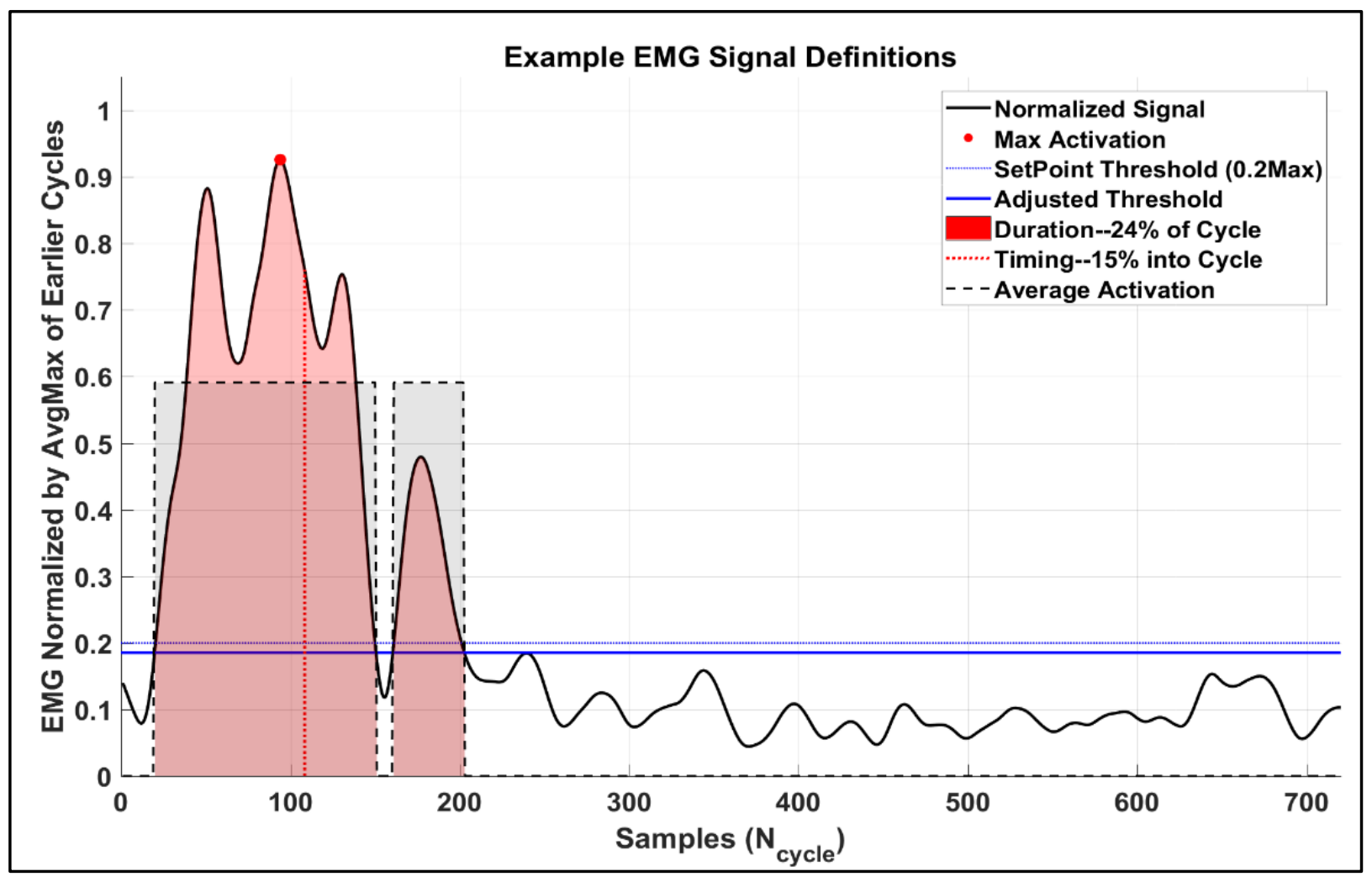

Figure 3-2: Schematic of example EMG signal thresholding and parameters measured.

Calculation of average activation for the cycle was done using (6):

$$
\text { Average }_{\text {active }, c}=\frac{\sum_{i=1}^{N_{\text {samples }, c}} E_{E G_{\text {norm }, c, i}\left(\text { Active }_{c, i}=1\right)}}{N_{\text {samples }, c}}
$$

Calculation of activation duration for the cycle was done using (7):

$$
\text { Duration } \text { active,c }=\frac{\sum_{i=1}^{N_{\text {samples }, c}}{ }_{\left(\text {Active }_{c, i}=1\right)}}{N_{\text {samples }, c}}
$$

Combining average activation and activation duration, a measure the "muscle energy" can be derived. Activation "energy" was calculated by the product of average activation and duration (8):

$$
\text { Energy }_{\text {active,c }}=\text { Average }_{\text {active,c }} * \text { Duration }_{\text {active,c }}
$$

Lastly, median timing of the muscle activation pulses was calculated using (9). It is important to note that the definition for timing is insensitive to multiple-pulses within a given cycle and would be unable to distinguish centered single-pulse activation from centered bi-modal activation. 


$$
\operatorname{Timing}_{\text {active }, \mathrm{c}}=\frac{\sum_{i=1}^{N_{\text {samples }, c}{ }_{i *\left(\text { Active }_{c, i}=1\right)}}}{N_{\text {samples }, \mathrm{c}}}
$$

Two additional consolidated metrics were calculated for each pair of unilateral parameters allow simplification of the final model form. "Mean" value was calculated as the average parameter value between subsequent right-left cycles (10). "Symmetry" was calculated as the difference between subsequent right-left values, normalized by the respective "Mean" value (11).

$$
\begin{gathered}
\text { Mean } \\
\text { active }, c \\
=\frac{\text { Parameter }_{\text {right }, c}+\text { Parameter }_{\text {left }, c}}{2} \\
\text { Symmetry }_{\text {active }, c}=\frac{\mid \text { Parameter }_{\text {right }, c}-\text { Parameter }_{l e f t, c \mid} \mid}{M e a n_{\text {active }, c}}
\end{gathered}
$$

\subsubsection{FAtigue Model DeVElopMENT}

In summary from Section 3.2.2, muscle parameters used include:

- Max Activation Signal (Normalized Calibration Max)

- Average Activation Signal (Normalized to Calibration Max)

- Activation Duration (Proportion of Cycle)

- Activation Timing (Proportion into Cycle)

- Activation "Energy"

- Bilateral Mean Parameter Value

- Bilateral Parameter Symmetry Value

Definitions for the above parameters can be found in the previous section (3.3.3). Each of the five parameters are measured bilaterally for the tibialis anterior, medial gastrocnemius, and rectus femoris. Prior to parameter calculation, EMG signals were linearly interpolated to define EMG signal strength at 0.5-degree crank angle increments over the cycle. Use of dynamic thresholding allowed normalization of parameters across subjects and performance types. To equally weight each subject in each dataset for model development, the parameter histories across the ride segment 
were linearly interpolated to 500 steps at an interval of $0.2 \%$ of ride segment. An overview of the model development pipeline is provided [Figure 3-3] with details below.

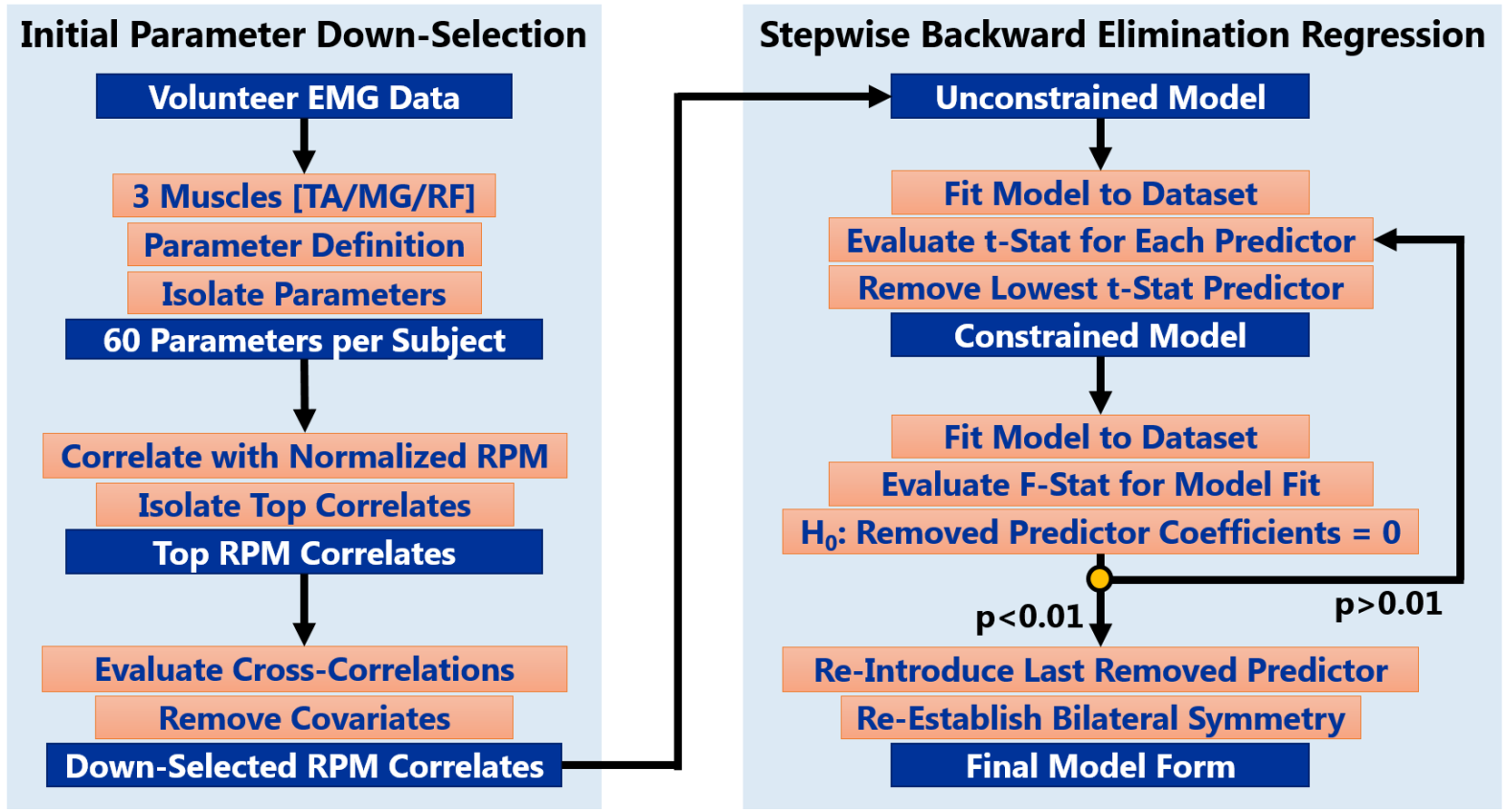

Figure 3-3: EMG-based Model Development Pipeline

\section{Model Training Dataset Selection}

From the performance description analyses conducted in Chapter 2, three classes of overall performance during the trial were found: "Non-Fatigue", "Moderate Fatigue", and "Fatigue". Since the change in performance level (RPM) during each isokinetic region is of interest, only subjects exhibiting significant performance loss will be used for development of the model structure. Therefore, categorization of the subjects in the "Fatigue" group is reevaluated base on the proportion of RPM decrease over the selected isokinetic region. "Non-Fatigue" subjects who exhibit moderate RPM decreases will also be removed from consideration in this analysis.

\section{Fatigue Model Structure}

For simplicity and minimal computational cost, a linear regression model has been used. Input parameters are selected from a subset of EMG measures. The model response variable was normalized RPM, which was normalized to the average RPM of the subject's first 25 cycles during the isokinetic section of the endurance ride. 


\section{Predictor Parameter Selection}

From the 60 total potential EMG parameters to be included per subject per cycle (5 parameters/muscle x 3 muscles x 3 right/left/mean/symmetry), a subset of parameters with the highest correlative strength with RPM were selected. To do so, univariate linear regression correlations were run between each input parameter and the model response. From these correlations, the strong correlates were isolated using a Student's t-Test approach. Since mean $\mathrm{R}^{2}$ values between the performance types differed, use of a fixed threshold would lead to a large difference in initial model sizes. Parameters with significantly higher correlation values were isolated for initial model fitting ( $\mathrm{p}>0.01$ ). However, prior to model fitting, covariates needed to be removed from the parameter sample. Cross correlations between parameters were calculated, a list of parameters sorted by RPM correlation was defined. Covariates were removed by iteratively stepping down the list and deleting high cross-correlation parameters. Through this process, a small sub-sample of independent high-RPM correlates was defined for each dataset. Model parameter symmetry was established prior to fitting analyses.

Evaluation of model fit was conducted through a stepwise backward elimination regression analysis. At each iteration, the lowest t-statistic parameter from the prior model fitting was removed (12). The F-statistic was used as a criterion (at $\alpha=0.01$ ) to determine when a significant decrease in constrained model fit occurred (13). To visualize model correlation strength, the adjusted $\mathrm{R}^{2}$-value was tracked over backward elimination steps (14).

$$
t_{\widehat{\beta}}=\frac{\widehat{\beta}-\beta_{0}}{\text { s.e. }(\widehat{\beta})}
$$

Where $\hat{\beta}$ is the predicted parameter coefficient, $\beta_{0}$ is the tested parameter coefficient (in this case $0)$, and s.e. $(\hat{\beta})$ is the standard error of the coefficient estimate for a given model fit.

$$
F_{J, N-K-1}=\left(R_{u}^{2}-R_{C}^{2}\right) * \frac{N-K-1}{\left(1-R_{u}^{2}\right) * J}=\frac{\frac{\left(S S E_{c}-S S E_{u}\right)}{J}}{\frac{S S E_{u}}{N-K-1}}=\frac{\left(S S E_{c}-S S E_{u}\right) *(N-K-1)}{S S E_{u} * J}
$$


Where $R_{u}^{2}$ and $R_{c}^{2}$ are the unconstrained and constrained correlation coefficients, respectively. $N$ is the number of samples, $K$ is the number of model parameters, and $J$ is the number of restrictions in the model. $N-K-1$ is the unrestricted degrees of freedom $\left(d f_{u}\right)$, and $J$ is equivalent to $d f_{c}-$ $d f_{u}$. For these calculations, the $S S E$ for was used, where $S S E_{u}$ and $S S E_{c}$ are unconstrained and constrained model fit sum of square errors, respectively.

$$
R_{\text {adjusted }}^{2}=1-\left[\frac{\left(1-R^{2}\right)(N-1)}{N-K-1}\right]
$$

Adjustment of $R^{2}$ was done to penalize for model size. However, since the model is fit to many samples $(N)$ relative to the number of parameters $(K)$, the $R_{\text {adjusted }}^{2} \cong R^{2}$. Final root mean square error (RMSE) and F-statistic values are presented for the grouped dataset model fits.

\section{Additional Model Considerations}

Once the model form was reduced through the stepwise elimination analysis, model symmetry was reestablished. Without information on volunteer leg dominance or how leggedness is implicated in cycling performance, the presented models are bilaterally symmetric. Reincorporated unilateral parameters are identified in the results presented below.

\subsubsection{Fatigue Model Evaluation}

To evaluate the developed group dataset models, the model forms were implemented to subjectspecific data in two ways. First, the final model form and coefficients from the grouped datasets were used to predict RPM performance on the original non-interpolated EMG data for each subject's isokinetic section. Residual probability density histograms and RMSE were calculated each combination of volunteer dataset (x6) and model form (x3) allowed for direct comparison of model types in their ability to predict a given subject's performance in the original response units [Section 3.3.3]. Next, the model form's ability to predict new observations was evaluated. Calculation of the prediction error sum of squares (PRESS) and the prediction- $R^{2}$ were implemented on the interpolated 500-sample subject-specific datasets used for initial model fitting. Though PRESS can be calculated via the hat-matrix $(h)$, direct iteration of model fitting and sample prediction was used to evaluate PRESS residuals $\left(P R E S S_{i}\right.$ or $\left.\hat{e}_{(i)}\right)$ and PRESS $(15,16)$. 


$$
\operatorname{PRESS}_{i} \text { or } \hat{e}_{(i)}=\frac{\hat{e}_{i}}{1-h_{i i}}
$$

Where $\hat{e}_{i}$ is the ordinary residual, and $h_{i i}$ is the $i^{t h}$ diagonal of the hat-matrix, defined as $H=$ $X\left(X^{\prime} X\right)^{-1} X^{\prime}$ for a given $N$-observation $x P$-predictor matrix, $X$. Iteratively, $\hat{e}_{(i)}$ can also be found by removing the $i^{\text {th }}$ observation from the dataset, re-fitting the model, predicting the removed observation, and calculating the residual. In effect, calculation of $\hat{e}_{(i)}$ treats the removed observation as a new input outside of the dataset the model was fit to. Summation of squared PRESS residuals allows for calculation of PRESS.

$$
\text { PRESS }=\sum_{i=1}^{N} \hat{e}_{(i)}^{2}
$$

From the PRESS statistic, the prediction $R^{2}$ value can be calculated simply (17).

$$
R_{\text {prediction }}^{2}=1-\frac{\text { PRESS }}{\text { Total Sum of Squares }}
$$

Where the total sum of squares (TSS or SST) is defined as the sum of all squared observationmean residuals in a dataset. Results from these model development, fitting, and evaluation techniques are presented in the following section. 


\subsection{RESULTS}

\subsubsection{Model TRAINING DATASETS}

Once RPM over the selected isokinetic regions was normalized to the initial RPM, each of the previously categorized "Fatigue subjects" were re-evaluated [Figure 3-4, next page]. Through reevaluation of the isokinetic RPM profiles, subject 2639 was removed from the "Fatigue" performance category. This leaves leaving three subjects $(4218,8105,9452)$ for development of the fatigue model structure. All three "Non-Fatigue" individuals exhibited an average increase in RPM over their isokinetic regions, and will be used to develop a "Non-Fatigue" model, as well as test applicability of the fatigue model to different performance types. In summary, the following model types were developed [Table 3-1]:

Table 3-1: Models used to Evaluate Non-Fatigue Performance Prediction

\begin{tabular}{|c|c|c|}
\hline Model Type & $\begin{array}{c}\text { Average RPM Change } \\
\mathbf{\pm} \text { Standard Error }\end{array}$ & Data Used to Fit Coefficients \\
\hline All Fatigue & $-42.5 \% \pm 0.3 \%$ & Fatigue Subjects: $4218-8105-9452$ \\
\hline All Non-Fatigue & $+5.6 \% \pm 0.2 \%$ & Non-Fatigue Subjects: $2786-5121-7924$ \\
\hline General (Combined) & --- & All Subjects Above \\
\hline
\end{tabular}




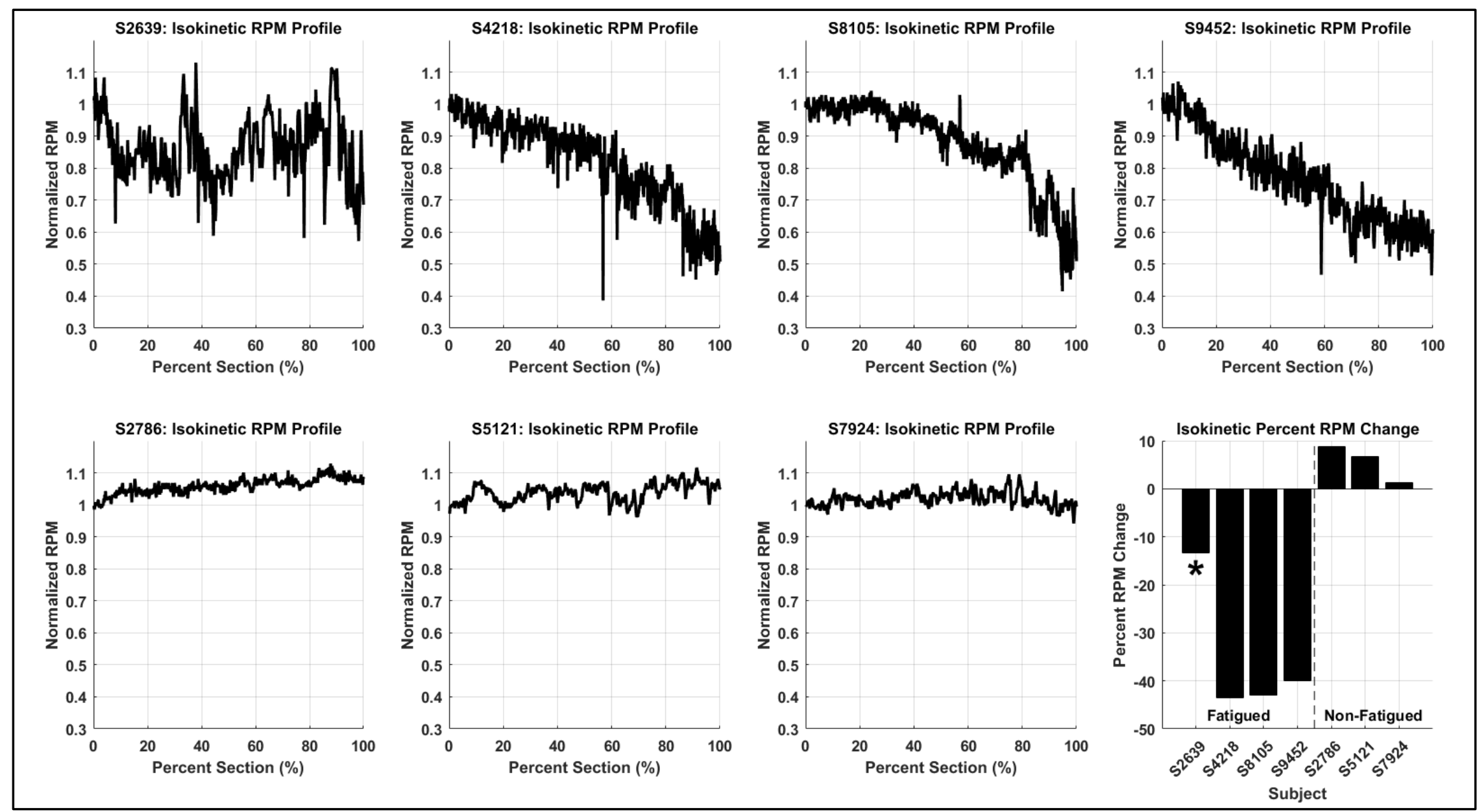

Figure 3-4: Normalized RPM Profiles for Reevaluation of "Fatigue" and "Non-Fatigue" Categorization. Summary percent RPM change for the subjects (bottom-right) with asterisk indicating statistically different "Fatigue" subjects $(\mathrm{p}<0.05)$. 


\subsubsection{UniVARIATE CORRELATIONS AND MODEL DEFINITIONS}

Selection of muscle parameters to include in linear regression analyses was done through comparison of univariate correlations with the chosen model response, subject's normalized RPM. Correlation coefficients have been categorized, color coded, and presented below [Figure 3-5].

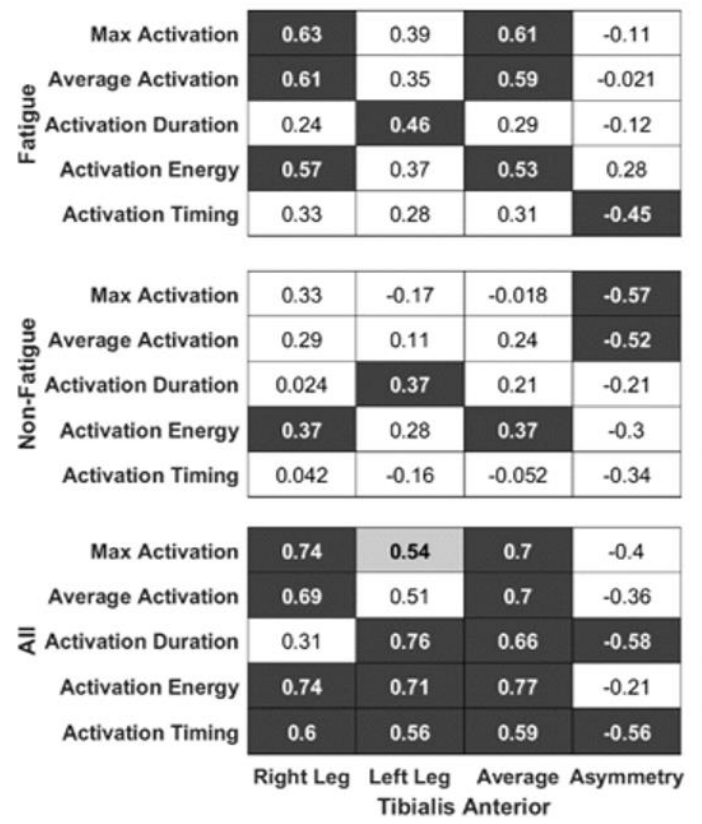

\begin{tabular}{|c|c|c|c|}
\hline 0.71 & 0.19 & 0.67 & -0.53 \\
\hline 0.71 & 0.33 & 0.67 & -0.54 \\
\hline 0.21 & -0.37 & 0.013 & -0.36 \\
\hline 0.55 & 0.14 & 0.46 & -0.48 \\
\hline-0.32 & -0.3 & -0.33 & 0.2 \\
\hline & & & \\
\hline 0.59 & -0.28 & 0.36 & -0.38 \\
\hline 0.66 & -0.25 & 0.25 & -0.41 \\
\hline-0.54 & -0.19 & -0.48 & -0.56 \\
\hline-0.094 & -0.41 & -0.32 & -0.56 \\
\hline-0.034 & -0.018 & -0.02 & -0.53 \\
\hline \multicolumn{4}{|c|}{} \\
\hline 0.8 & 0.059 & 0.72 & -0.59 \\
\hline 0.84 & 0.43 & 0.8 & -0.57 \\
\hline 0.027 & -0.74 & -0.44 & -0.38 \\
\hline 0.63 & -0.16 & 0.4 & -0.58 \\
\hline-0.3 & -0.29 & -0.31 & 0.35 \\
\hline Right Leg & Left Leg & Average Asymmetry \\
\hline Medial Gastrocnemius & \\
\hline
\end{tabular}

\begin{tabular}{|c|c|c|c|}
\hline 0.34 & 0.34 & 0.43 & -0.2 \\
\hline 0.42 & 0.39 & 0.5 & -0.25 \\
\hline 0.28 & -0.067 & 0.19 & 0.033 \\
\hline 0.36 & 0.57 & 0.51 & -0.21 \\
\hline-0.068 & -0.11 & -0.091 & 0.28 \\
\hline \multicolumn{4}{|c|}{} \\
\hline-0.29 & 0.41 & 0.22 & -0.021 \\
\hline 0.23 & 0.31 & 0.35 & -0.44 \\
\hline-0.44 & -0.25 & -0.49 & -0.38 \\
\hline-0.44 & -0.016 & -0.24 & -0.51 \\
\hline 0.16 & 0.15 & 0.16 & -0.071 \\
\hline \multicolumn{4}{|c|}{} \\
\hline 0.38 & 0.22 & 0.35 & -0.18 \\
\hline 0.41 & 0.12 & 0.3 & -0.35 \\
\hline 0.56 & 0.33 & 0.57 & -0.25 \\
\hline 0.56 & 0.46 & 0.62 & -0.4 \\
\hline 0.54 & 0.51 & 0.53 & -0.085 \\
\hline Right Leg & Left Leg & Average Asymmetry \\
\hline & Rectus Femoris \\
\hline
\end{tabular}

Figure 3-5: Univariate Correlation Coefficients and RPM Predictor Selection. Fatigue, nonfatigue and combined datasets (rows, top-to-bottom) were correlated with RPM reduction across Tibialis Anterior (left column), Medial Gastrocnemius, and Rectus Femoris muscle parameters (columns, left-to-right). Parameters with significant correlation to RPM change are indicated by gray $(\mathrm{p}<0.05)$ and black $(\mathrm{p}<0.01)$ boxes.

From the univariate correlations of each performance dataset, strong RPM correlates were found for regression model development. When the bilateral average value of the parameter showed a stronger correlation than either leg independently, only the bilateral average was included in the initial model. Although the presented method for parameter selection yielded a subset of strongly correlated parameters, adjustments to the final model parameters were made. First, a Student's tTest was run to identify high cross-correlation parameter pairs. Covariance significance for the initial parameters from univariate RPM correlations is presented [Figure 3-6]. 


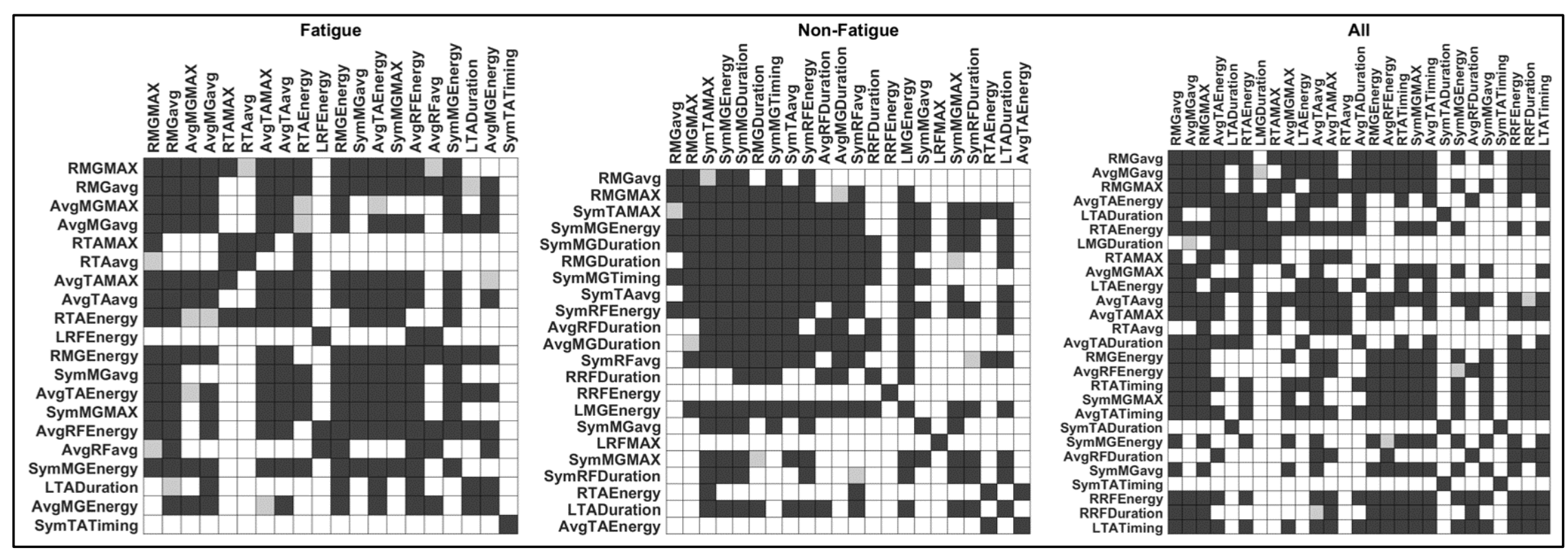

Figure 3-6: Covariance Matrices for Initial Set of Model Parameters. Parameters with significant covariances are indicated by gray $(\mathrm{p}<0.05)$ and black $(\mathrm{p}<0.01)$ squares, respectively. Significance was determined by a Student $\mathrm{t}$-test across top RPM correlates for each dataset. Parameters were sorted by decreasing $\mathrm{R}^{2}$-value with RPM from left-to-right, and top-to-bottom. 
To remove the identified covariates, the sorted parameter list was used to iteratively eliminate all significant covariates $(\mathrm{p}<0.01)$ to each parameter in order of descending RPM correlation. Since the dominant leg was not identified for these volunteers, parameters showing strong unilateral correlations were included in the model with both right and left leg parameters. Through this selection process, initial model parameters without cross correlations were found [Table 3-2].

Table 3-2: Initial Parameters Down-Selected from Covariant Removal

\begin{tabular}{|c|c|c|c|}
\hline $\begin{array}{c}\text { Performance Model } \\
\text { Type }\end{array}$ & Tibialis Anterior & $\begin{array}{c}\text { Medial } \\
\text { Gastrocnemius }\end{array}$ & Rectus Femoris \\
\hline $\begin{array}{c}\text { Fatigue Group } \\
\text { 9-parameters }\end{array}$ & $\begin{array}{c}\mathrm{R}+\mathrm{L}^{*} \text { Average } \\
\mathrm{R}^{*}+\mathrm{L} \text { Duration } \\
\text { Timing Symmetry }\end{array}$ & $\mathrm{R}+\mathrm{L}^{*} \mathrm{Max}$ & $\mathrm{R}^{*}+\mathrm{L}$ Energy \\
\hline $\begin{array}{c}\text { Non-Fatigue Group } \\
\text { 10-parameters }\end{array}$ & $\begin{array}{c}\text { Max Symmetry } \\
\text { Mean Energy }\end{array}$ & $\mathrm{R}+\mathrm{L}^{*}$ Average & $\begin{array}{c}\mathrm{R}^{*}+\mathrm{L} \text { Max } \\
\mathrm{R}+\mathrm{L}^{*} \text { Duration } \\
\mathrm{R}+\mathrm{L}^{*} \text { Energy }\end{array}$ \\
\hline $\begin{array}{c}\text { (Combined Dataset) } \\
\text { 8-parameters }\end{array}$ & $\begin{array}{c}\mathrm{R}+\mathrm{L}^{*} \text { Average } \\
\text { Duration Symmetry }\end{array}$ & $\begin{array}{c}\mathrm{R}+\mathrm{L}^{*} \text { Average } \\
\mathrm{R}^{*}+\mathrm{L} \text { Duration }\end{array}$ & Mean Duration \\
\hline
\end{tabular}

*Parameters included to maintain bilateral symmetry.

Therefore, initial model structures for the Fatigue, Non-Fatigue, and Combined datasets contained 9-parameters, 10-parameters, and 8-parameters, respectively. The initial model structure generated from these parameters was reduced through a stepwise backward elimination regression analysis. Reduction in adjusted $\mathrm{R}^{2}$-value of the model was tracked [Figure 3-7]. Model structures prior to a significant decrease in prediction strength were identified via iterative F-tests. 


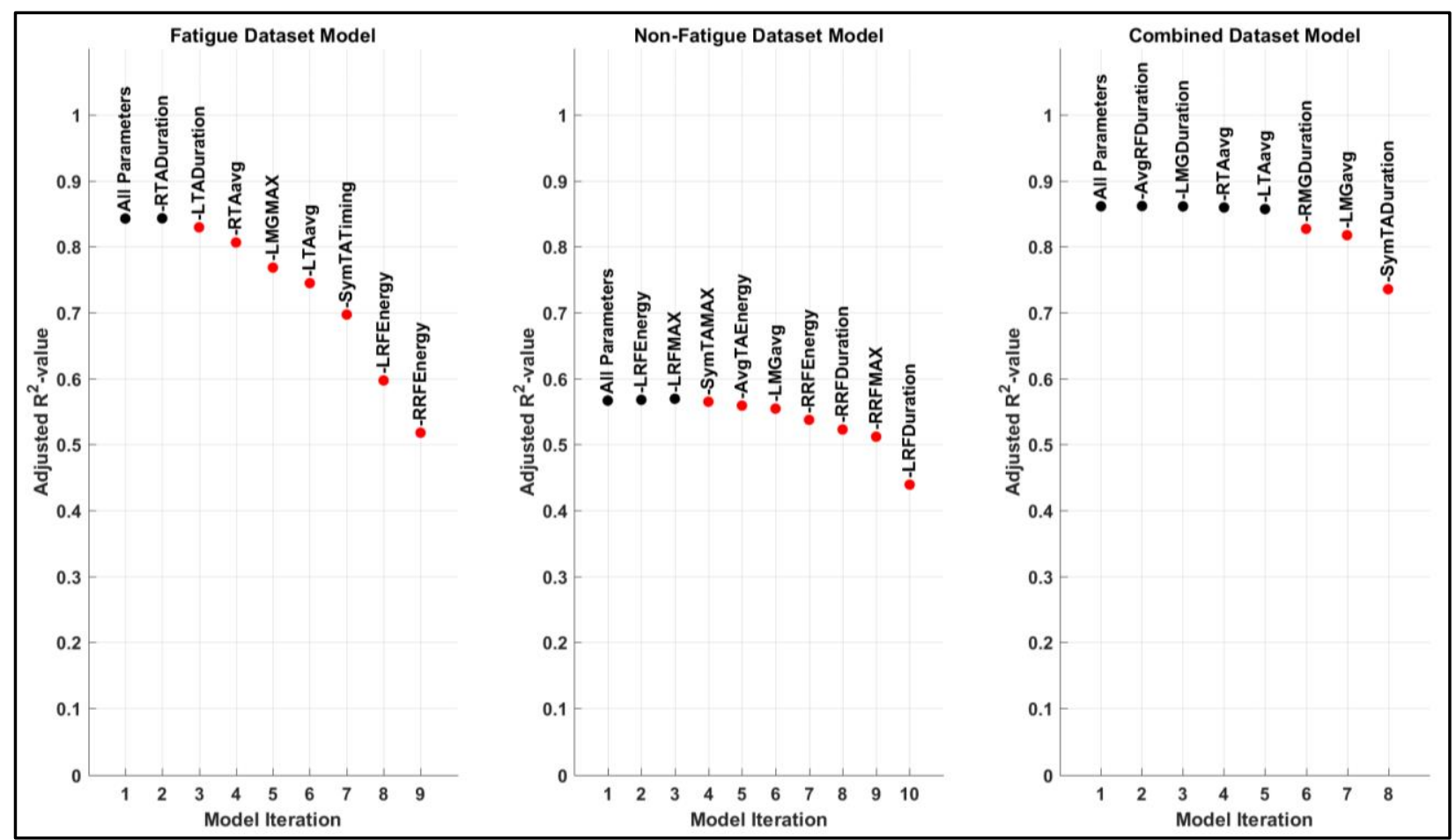

Figure 3-7: Change in Adjusted R2-value with Robust Stepwise Backward Elimination Regression Analysis. Models with a significant decrease in predictive strength are shown in red $(\mathrm{p}<0.01)$. The parameter removed from the prior iteration is noted above each model marker.

After a significant decrease in model fit was detected, the model was isolated. Before finalizing model form and coefficients, necessary parameters were re-introduced to preserve bilateral symmetry. The symmetric model form was re-fit to a subset of concatenated subject data and final model form and coefficients are presented [Table 3-3].

Table 3-3: Final Model Parameters from Stepwise Backward Elimination Regression Analysis

\begin{tabular}{|c|c|c|c|}
\hline $\begin{array}{c}\text { Performance Model } \\
\text { Type }\end{array}$ & Tibialis Anterior & $\begin{array}{c}\text { Medial } \\
\text { Gastrocnemius }\end{array}$ & Rectus Femoris \\
\hline $\begin{array}{c}\text { Fatigue Group } \\
\text { 9-Predictors } \\
\text { + Intercept }\end{array}$ & $\begin{array}{c}\text { R+L Average } \\
\text { R*+L Duration } \\
\text { Timing Symmetry }\end{array}$ & R+L Max & R+L Energy \\
\hline $\begin{array}{c}\text { Non-Fatigue Group } \\
\text { 10-Predictors } \\
\text { + Intercept }\end{array}$ & $\begin{array}{c}\text { Max Symmetry } \\
\text { Mean Energy }\end{array}$ & R+L Average & $\begin{array}{c}\text { R+L* Max } \\
\text { R+L Duration } \\
\text { R+L* Energy }\end{array}$ \\
\hline $\begin{array}{c}\text { Combined Dataset } \\
\text { 5-Predictors } \\
\text { + Intercept }\end{array}$ & Duration Symmetry & $\begin{array}{c}\text { R+L Average } \\
\text { R+L* Duration }\end{array}$ & --- \\
\hline
\end{tabular}

*Parameters re-introduced to maintain bilateral symmetry. 
Through the conducted model development, significant parameters were identified for each dataset. Model fit metrics are presented after each model's coefficients were optimized to a subset of the subject data [Table 3-4].

Table 3-4: Group Model Coefficients Ordered by Parameter Significance

\begin{tabular}{|c|c|c|c|c|c|c|}
\hline Model Type & Parameter & $\begin{array}{c}\text { Coefficient } \\
\text { Estimate }\end{array}$ & t-Stat & p-value & $\begin{array}{c}\text { Adjusted } \\
\mathbf{R}^{2}\end{array}$ & F-Stat \\
\hline \multirow{10}{*}{$\begin{array}{c}\text { Fatigue } \\
\text { Model } \\
\text { 9-Predictors } \\
\text { + Intercept }\end{array}$} & Intercept & 0.702 & 37.558 & $\mathrm{p}<<0.01$ & \multirow{10}{*}{0.843} & \multirow{10}{*}{$\begin{array}{c}892 \\
\frac{p \text {-value }}{p<<0.01}\end{array}$} \\
\hline & R RF Energy & -1.180 & -23.346 & $\mathrm{p}<<0.01$ & & \\
\hline & R MG Max & 0.253 & 21.365 & $\mathrm{p}<<0.01$ & & \\
\hline & TA Timing Sym. & -0.202 & -14.889 & $\mathrm{p}<<0.01$ & & \\
\hline & L MG Max & -0.129 & -14.539 & $\mathrm{p}<<0.01$ & & \\
\hline & L RF Energy & 0.599 & 13.621 & $\mathrm{p}<<0.01$ & & \\
\hline & R TA Average & 0.219 & 11.805 & $\mathrm{p}<<0.01$ & & \\
\hline & L TA Average & 0.228 & 11.530 & $\mathrm{p}<<0.01$ & & \\
\hline & L TA Duration & 0.715 & 9.729 & $\mathrm{p}<<0.01$ & & \\
\hline & R TA Duration & -0.056 & -2.409 & 0.016 & & \\
\hline \multirow{11}{*}{$\begin{array}{c}\text { Non-Fatigue } \\
\text { Model } \\
\text { 10-Predictors } \\
\text { + Intercept }\end{array}$} & Intercept & 0.998 & 67.663 & $\mathrm{p}<<0.01$ & \multirow{11}{*}{$\begin{array}{r}0.567 \\
\text { RMSE } \\
1.88 \%\end{array}$} & \multirow{11}{*}{$\begin{array}{c}197 \\
\frac{\mathrm{p} \text {-value }}{\mathrm{p}<<0.01}\end{array}$} \\
\hline & R MG Average & 0.257 & 15.874 & $\mathrm{p}<<0.01$ & & \\
\hline & R RF Max & -0.077 & -8.043 & $\mathrm{p}<<0.01$ & & \\
\hline & L RF Duration & -0.137 & -7.417 & $\mathrm{p}<<0.01$ & & \\
\hline & R RF Duration & -0.135 & -6.294 & $\mathrm{p}<<0.01$ & & \\
\hline & R RF Energy & 0.350 & 6.042 & $\mathrm{p}<<0.01$ & & \\
\hline & Mean TA Energy & 0.041 & 4.277 & $\mathrm{p}<0.01$ & & \\
\hline & TA Max Sym. & -0.012 & -3.064 & 0.002 & & \\
\hline & L MG Average & 0.039 & 3.036 & 0.002 & & \\
\hline & L RF Max & 0.012 & 2.087 & 0.037 & & \\
\hline & L RF Energy* & -0.034 & -0.834 & 0.404 & & \\
\hline \multirow{6}{*}{$\begin{array}{c}\text { General } \\
\text { Model } \\
\text { 5-Predictors } \\
\text { + Intercept }\end{array}$} & Intercept & 0.735 & 58.963 & $\mathrm{p}<<0.01$ & \multirow{6}{*}{0.856} & \multirow{3}{*}{3580} \\
\hline & R MG Average & 0.476 & 45.947 & $\mathrm{p}<<0.01$ & & \\
\hline & L MG Average & 0.507 & 39.892 & $\mathrm{p}<<0.01$ & & \\
\hline & TA Duration Sym. & -0.141 & -27.932 & $\mathrm{p}<<0.01$ & & p-value \\
\hline & R MG Duration & -0.464 & -22.596 & $\mathrm{p}<<0.01$ & & $\mathrm{p}<<0.01$ \\
\hline & L MG Duration* & -0.022 & -0.827 & 0.408 & & \\
\hline
\end{tabular}

*Predictor is not a statistically significant model input $(p>0.05)$.

Model parameters presented above are implemented in a simple linear model form:

$$
\text { RPM Ratio }=\text { Intercept }+\sum_{p=1}^{N_{\text {predictors }}}\left(\text { Coef }_{p} \times \text { Predictor }_{p}\right)
$$




\subsubsection{GROUPED MOdEL PREDICTIONS}

The above models were used to predict subject-specific normalized RPM profiles for the full isokinetic section of the tests [Figures 3-8 and 3-9, next two pages]. For each subject's figure, the normalized RPM profile is shown in black. Overlaying the experimental data, subject-specific model predictions, calculated with the "Fatigue"-fitted, "Non-Fatigue"-fitted, and "General"-fitted models, are shown in solid red, blue, and green lines, respectively. Residual histograms are also shown for the three models presented for qualitative comparisons of model fit to experimental data. The prediction's root mean square error (RMSE) was calculated to evaluate model fit to the input datasets [Table 3-5].

Table 3-5: Summary of RMSE from Group Model Predictions of Subject Data

\begin{tabular}{|c|c|c|c|c|}
\hline \multirow{2}{*}{$\begin{array}{l}\text { Subject } \\
\text { Group }\end{array}$} & \multirow{2}{*}{$\begin{array}{c}\text { Subject } \\
\text { Number } \\
\end{array}$} & \multicolumn{3}{|c|}{ RMSE by Grouped Model Type ( \pm Std. Error) } \\
\hline & & Fatigue & Non-Fatigue & General \\
\hline \multirow{3}{*}{ Fatigue } & 4218 & $9.74 \%$ & $22.1 \%$ & $6.45 \%$ \\
\hline & 8105 & $3.30 \%$ & $16.5 \%$ & $4.60 \%$ \\
\hline & 9452 & $5.47 \%$ & $19.4 \%$ & $14.6 \%$ \\
\hline \multirow{3}{*}{ Non-Fatigue } & 2786 & $24.9 \%$ & $1.67 \%$ & $3.76 \%$ \\
\hline & 5121 & $22.1 \%$ & $2.23 \%$ & $3.11 \%$ \\
\hline & 7924 & $9.70 \%$ & $1.52 \%$ & $3.96 \%$ \\
\hline \multicolumn{2}{|c|}{ Average Fatigue Group } & $6.17 \pm 1.89 \%$ & $19.3 \pm 1.60 \%$ & $8.56 \pm 3.08 \%$ \\
\hline \multicolumn{2}{|c|}{ Average Non-Fatigue Group } & $18.9 \pm 4.66 \%$ & $1.81 \pm 0.215 \%$ & $3.61 \pm 0.257 \%$ \\
\hline
\end{tabular}




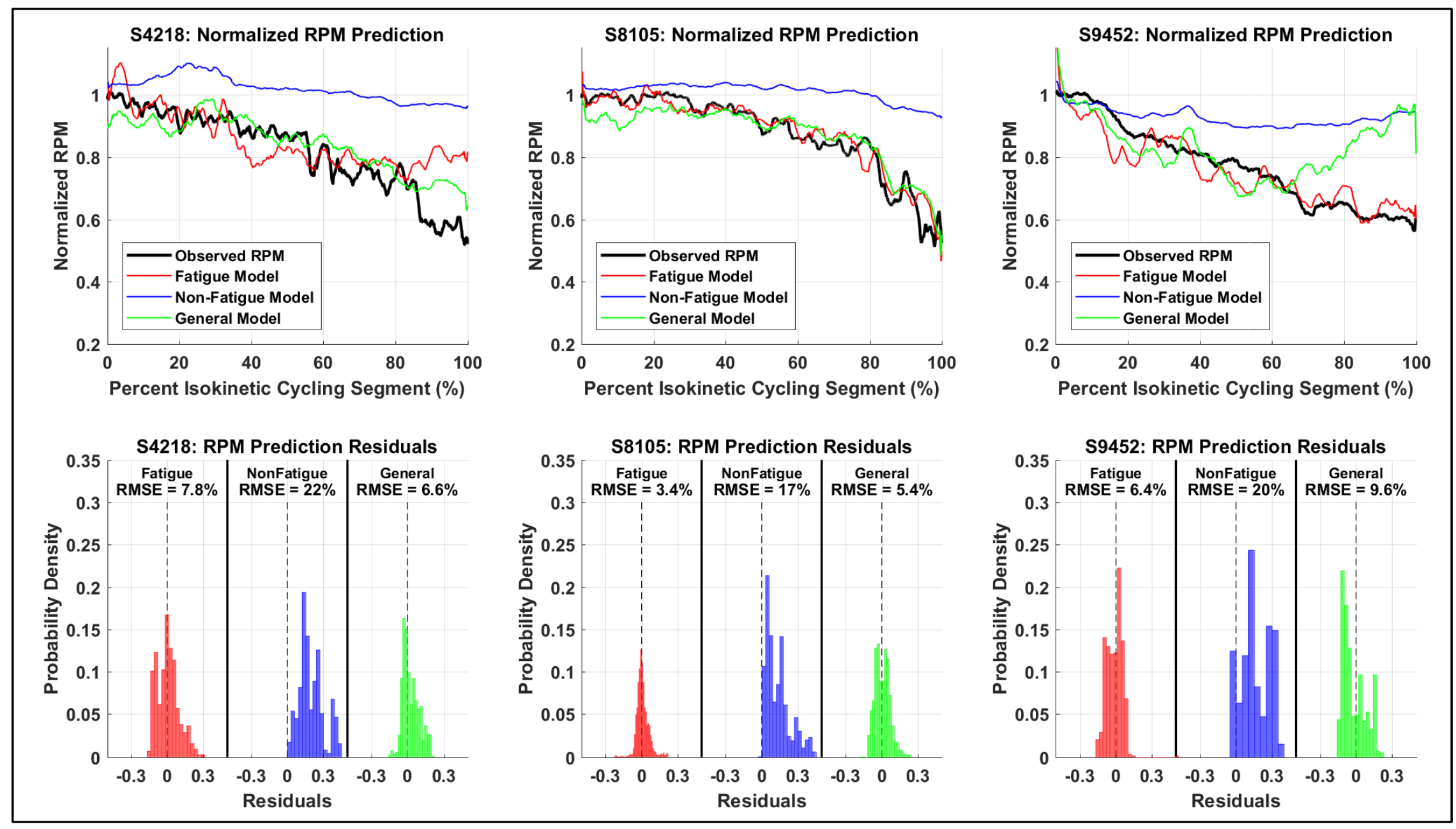

Figure 3-8: Fatigue Group Model Predictions and Residual Probability Density Plots. 


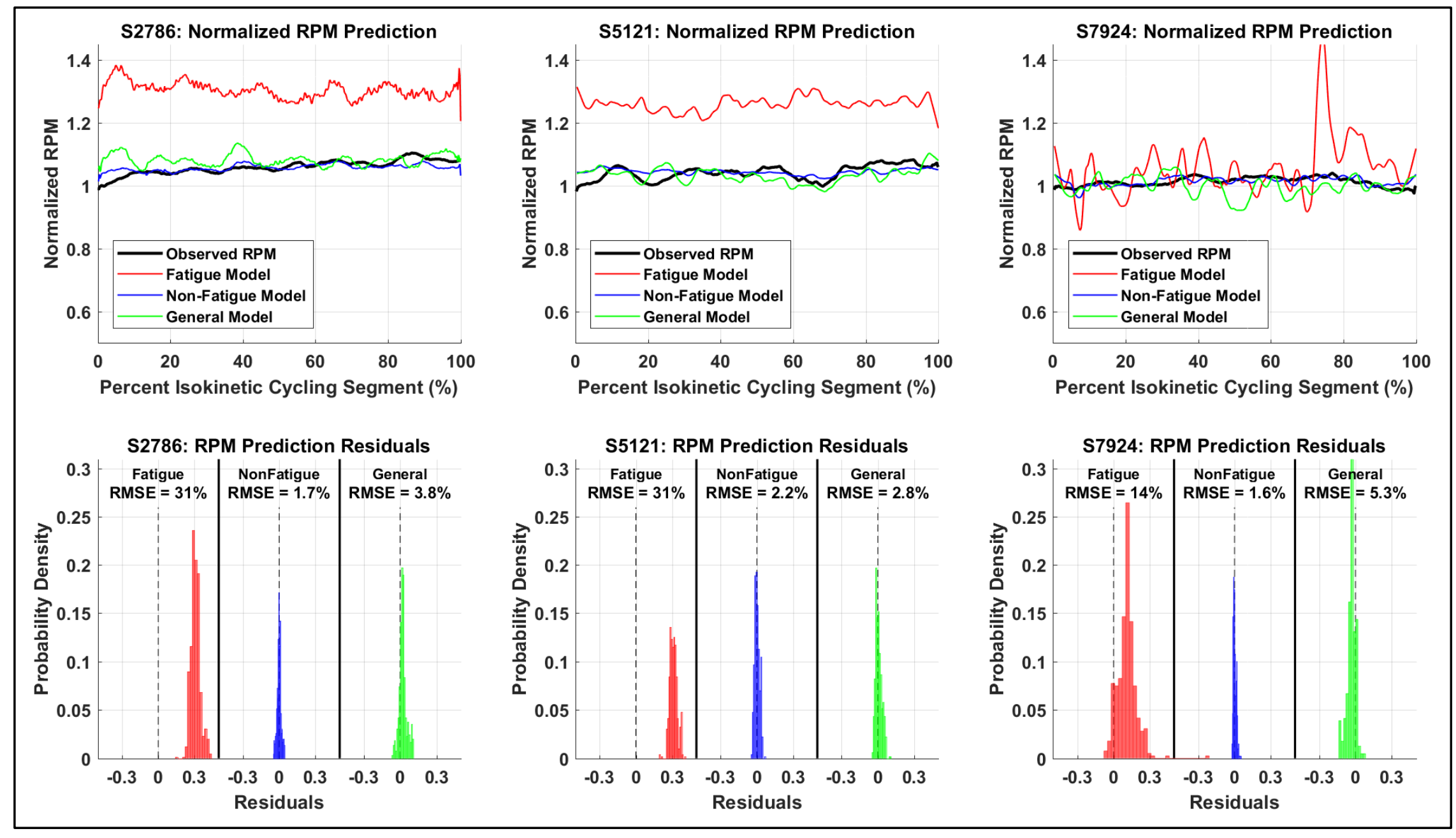

Figure 3-9: Non-Fatigue Group Model Predictions and Residual Probability Density Plots. 


\subsubsection{SUBJECT-SPECIFIC FITTING AND NEW OBSERVATIONS}

While Section 3.3.3 focused on model development for grouped datasets, this section evaluates subject-specific fitting of the group-model structures, and the ability for these models to make predictions on new observations. Through prediction error sum of squares (PRESS) analysis, metrics of a model's ability to predict new values were found. These metrics included calculation of PRESS [Table 3-6] and Prediction $\mathrm{R}^{2}$-value [Figure 3-11, next page], for each model-dataset.

Table 3-6: Summary of PRESS from Subject-Specific Fitting of Group Models

\begin{tabular}{|c|c|c|c|c|}
\hline $\begin{array}{c}\text { Subject } \\
\text { Group }\end{array}$ & $\begin{array}{c}\text { Subject } \\
\text { Number }\end{array}$ & \multicolumn{3}{|c|}{ PRESS by Grouped Model Type } \\
\hline \multirow{3}{*}{ Fatigue } & 4218 & 0.912 & 0.713 & General \\
\cline { 2 - 5 } & 8105 & 0.194 & 0.102 & 0.292 \\
\cline { 2 - 5 } & 9452 & 0.771 & 0.394 & 0.867 \\
\hline \multirow{3}{*}{ Non-Fatigue } & 2786 & 0.105 & 0.084 & 0.125 \\
\cline { 2 - 5 } & 5121 & 0.077 & 0.062 & 0.213 \\
\cline { 2 - 5 } & 7924 & 0.092 & 0.071 & 0.076 \\
\hline
\end{tabular}

Note: Subject-specific Non-Fatigue models yielded lowest PRESS-value across subjects. For reference, with 500 observations per subject, a PRESS value of 1 corresponds to an average prediction error of $\sim 4.47 \%$ of initial RPM.

Figure 3-10 provides the conversion between calculated PRESS-values and the corresponding average prediction error for a sample size of 500 observations used in the PRESS calculation.

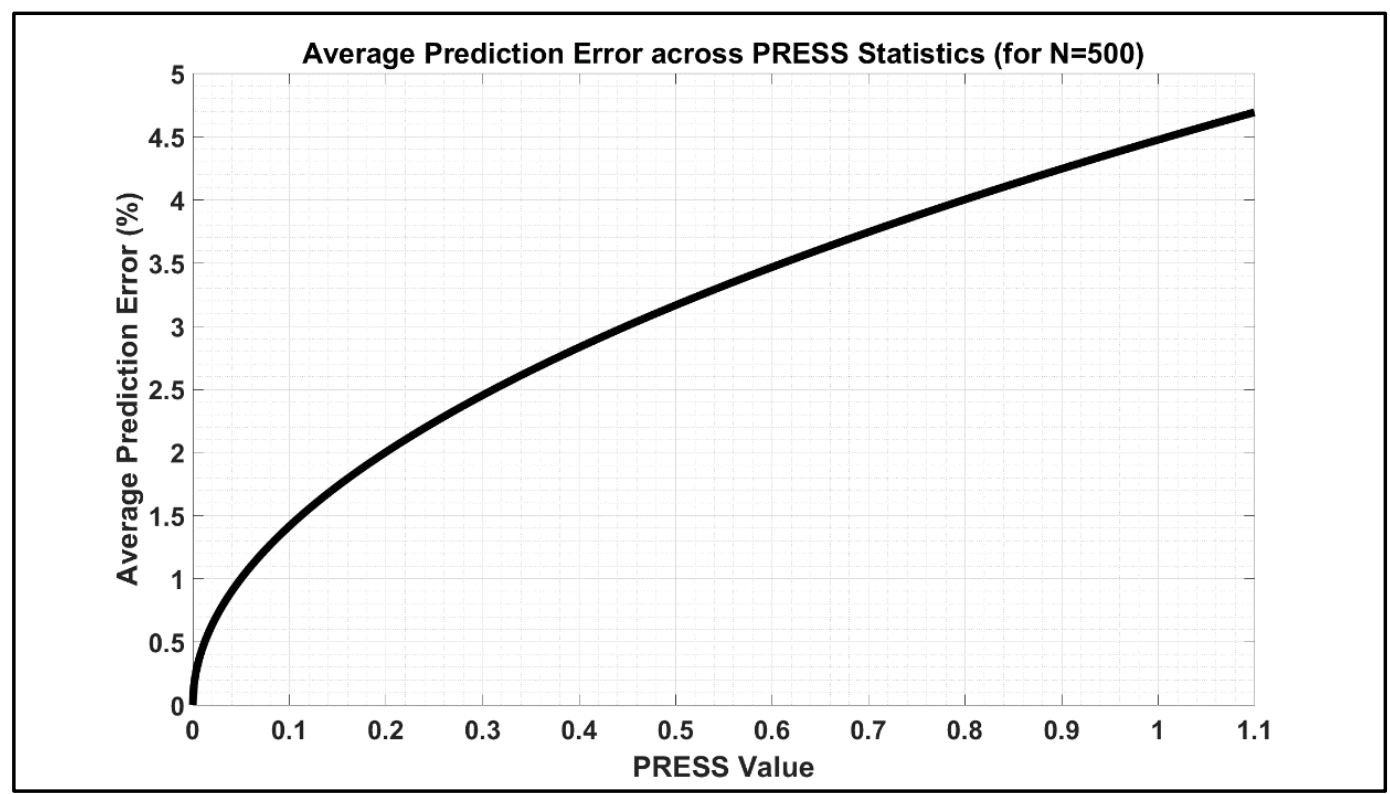

Figure 3-10: Conversion from PRESS to Average Prediction Error for $\mathrm{N}=500$ Observations 


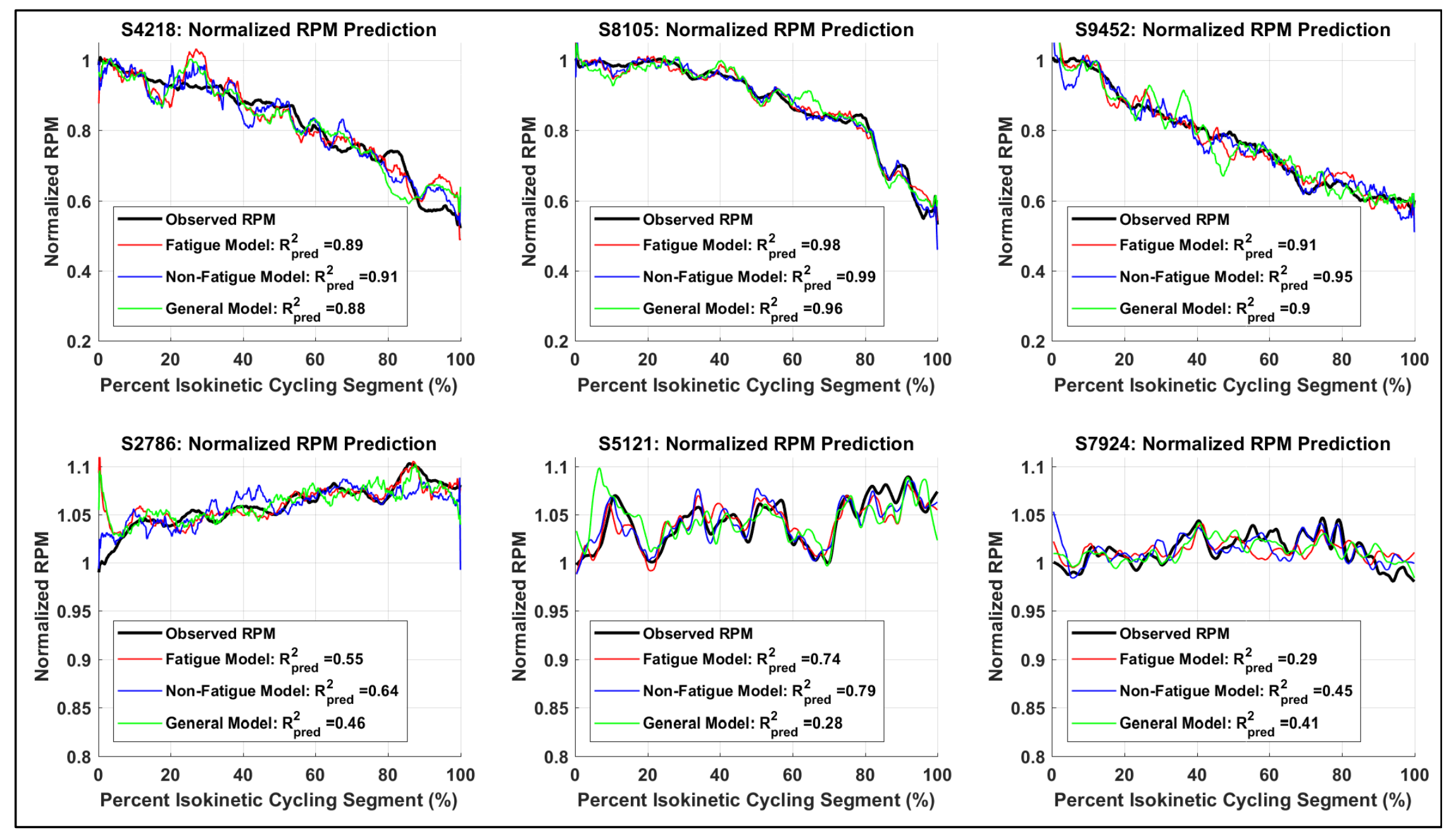

Figure 3-11: Prediction $\mathrm{R}^{2}$-values and Predicted Response from PRESS Calculations 
Table 3-7: Summary of Prediction $\mathrm{R}^{2}$ from Subject-Specific Fitting of Group Models

\begin{tabular}{|c|c|c|c|c|c|c|c|}
\hline \multirow{2}{*}{$\begin{array}{c}\text { Subject } \\
\text { Group }\end{array}$} & \multirow{2}{*}{$\begin{array}{c}\text { Subject } \\
\text { Number }\end{array}$} & \multicolumn{3}{|c|}{$\mathbf{R}^{\mathbf{2}}$ Pred and $\mathbf{R}^{\mathbf{2}}$ Pred-Adj by Grouped Model Type } \\
\cline { 3 - 8 } & $\mathbf{R}^{\mathbf{2}}$ Pred & $\mathbf{R}^{\mathbf{2}}$ Pred-Adj & $\mathbf{R}^{\mathbf{2}}$ Pred & $\mathbf{R}^{\mathbf{2}}$ Pred-Adj & $\mathbf{R}^{\mathbf{2}}$ Pred & $\mathbf{R}^{\mathbf{2}}$ Pred-Adj \\
\hline \multirow{4}{*}{ Fatigue } & 4218 & 0.890 & 0.887 & 0.914 & 0.912 & 0.877 & 0.875 \\
\cline { 2 - 8 } & 8105 & 0.975 & 0.975 & 0.987 & 0.987 & 0.963 & 0.963 \\
\cline { 2 - 8 } & 9452 & 0.911 & 0.909 & 0.954 & 0.953 & 0.900 & 0.898 \\
\hline \multirow{3}{*}{ Non-Fatigue } & 2786 & 0.551 & 0.542 & 0.642 & 0.634 & 0.464 & 0.458 \\
\cline { 2 - 8 } & 5121 & 0.740 & 0.735 & 0.789 & 0.785 & 0.278 & 0.269 \\
\cline { 2 - 8 } & 7924 & 0.286 & 0.271 & 0.454 & 0.441 & 0.408 & 0.401 \\
\hline
\end{tabular}

Note: Adjustment of Prediction $\mathrm{R}^{2}$ does not significantly alter conclusions from model predictions.

\subsection{DISCUSSION AND CONCLUSIONS}

\subsubsection{MODEL FORM AND COEFFICIENTS}

EMG signal morphology was analyzed through the endurance ride. Implementation of dynamic thresholding allowed for signal features to be detected, even at significantly diminished amplitudes. Prior to model development, the subject-specific and grouped datasets were defined (3.3.1). Two equally weighted datasets for the Non-Fatigue and Fatigue groups were set, as well as a combined "General" dataset to assess common parameters and model forms between the two performance groups. Univariate correlations of parameters with observed RPM and Student's tTests successfully identified strong performance predictors. Removal of covariates significantly reduced initial model size down to nine, ten, and eight parameters for the initial Fatigue, NonFatigue, and General model forms, respectively. Through stepwise backward elimination regression analysis, final model structures for each performance type were identified. While not a robust measure of model fit, the adjusted $R^{2}$ values for the Fatigue and General models remained above 0.8 , while remaining above 0.5 for the Non-Fatigue model. The observed lower $R^{2}$ values for Non-Fatigue correlations is likely due to the low magnitude RPM change relative to the Fatigue group. Through the model development process, three EMG-based performance model structures were formed. Final models contained 9, 10, and 5 predictors (and intercept) for the Fatigue, NonFatigue, and General models, respectively. 
All models exhibited RMSE values of less than 5\%, indicating strong fit of the model predictions to their training performance data. The final Fatigue group model shows relative importance of TA metrics that are positively correlated with overall performance, specifically: TA average activation, TA activation duration, and TA activation timing symmetry. However, stronger predictive strength was seen in RF activation energy and MG maximum activation. Similarly, the Non-Fatigue group shows MG and RF parameters are driving RPM performance prediction. The TA metrics are not prevalent in the Non-Fatigue model, with only mean TA activation energy and TA maximum activation level symmetry weighing significantly into RPM performance. The relative lack of TA contributions in Non-Fatigue group performance may indicate strong influence of TA weakness in driving performance decline. The hypothesis of TA importance in cycling performance is also substantiated by positive correlations of TA parameters in the Fatigue group. These correlations indicate that bilaterally maintaining the TA's average activation and activation duration and timing symmetry may effectively stave off performance reduction in long-duration cycling as fatigue develops.

From the combined dataset, the General model could maintain an RMSE of less than 5\% while converging to a 5-predictor performance model. It is important to note that the General model form does not include any RF metrics, and TA contributions are limited to activation duration symmetry. The remaining 4 predictors are bilateral MG average activation level and activation duration. The ability for this model to fit both, Fatigue and Non-Fatigue, performance types while maintaining a low RMSE hints towards a key role of the MG in guiding overall performance level, not just performance loss. While the RF appears to be a foundational muscle in defining performance level in both groups, results from initial model form shows that the combination of TA and MG muscle groups weigh heavily in the maintenance and reduction of performance in stationary cycling.

\subsubsection{MODEL FIT}

Evaluation of each model fit to each subject-specific dataset was quantified through calculation of RMSE and visualization of residual probability density histograms. In 5 of the 6 subjects, the model developed for the subject's performance type resulted in the lowest RMSE and tightest residual distribution. Predictions from the opposite model type tended to overpredict the performance level or not predict the magnitude of performance reduction. When predicting Fatigue 
subject performance with the Non-Fatigue model, the model under predicted the extent of performance loss. However, when predicting Non-Fatigue subject performance with the Fatigue model, the model overpredicted performance and did not capture the increases in performance during the Non-Fatigue ride section. By comparing the model forms, this discrepancy may be attributed to the effect of TA dynamics on model predictions. In the Fatigue model, TA dynamics play a significant role in performance change, but the Non-Fatigue subject's performance increase does not exhibit TA changes. Therefore, the Fatigue model is unable to predict overall RPM increases. Likewise, the Non-Fatigue model has low TA contributions to predictive strength. Therefore, when this model is used for Fatigue subjects that exhibit significant decreases in TA activation metrics (namely, activation level, duration, and timing symmetry), the model is unable to track well with the observed performance loss.

The General model provided an intermediate level of subject-specific fit, often exhibiting RMSE values only slightly greater than the model developed for the subject's respective performance type. While only implementing five MG and TA metrics, each of these metrics (or a strong covariate) were found among the top predictors in both the Fatigue and Non-Fatigue groups. Drawing from both performance group characteristics, the General model also shows some weaknesses carried over from the performance-specific models, which are typically observed in greater residual range and minor over estimation. Through this phase model evaluation, the General model form, once trained on a large dataset, offers a robust and flexible predictor for both stages of stationary cycling performance. In addition, the model's focus on MG dynamics while exhibiting a low RMSE fit indicates strong contributions of the MG in overall performance.

\subsubsection{MOdEL PREDICTION}

Though initial evaluation of the developed models indicates strong fit of the performance-specific and General models to the observed RPM profiles, strong model fit does not directly translate to a model's ability to predict new observations. Calculation of the PRESS and Prediction $R^{2}$-value allow for a quantitative evaluation of the models' subject-specific predictive strength. Maximum observed PRESS was 1.02, indicating that all average prediction errors were found to be less than $5 \%$ of initial RPM. Across all both performance types, the Non-Fatigue model form yielded the strongest predictive strength, with an average PRESS of 0.238 and a less than $2.5 \%$ average 
prediction error. The predictive performance of this model form is likely attributed to the larger model size, which this formulation of PRESS does not penalize for. While the General model form yielded a response fit that was flexible to both performance types, the predictive strength of this model form is low compared to the Fatigue and Non-Fatigue forms, even when the performancespecific models are used to predict subject performance from the opposite group. Like the advantage given to the Non-Fatigue model, the General model's PRESS values may suffer from small model size, and therefore reduced range of flexibility in subject-specific fit and prediction. Also, it is seen that all models performed better on the Non-Fatigue subjects than on the Fatigue group. This difference is likely due to the larger range of performance seen in the Fatigue group, which demands more predictive robustness of the model form and coefficients. While a range of PRESS values are exhibited by the models, all observed average prediction errors fall between $1 \%$ and 5\%, which are similar to the RMSE magnitudes seen in the fit evaluation section (3.3.3).

Evaluation of prediction $R^{2}$ shows a similar trend when comparing model-to-model predictive strength. The Non-Fatigue model form yielded the highest $R_{\text {pred }}^{2}$-values while the General model form yielded the lowest. However, the model performance had variation from subject to subject. Contrasting the PRESS statistic results, the prediction $R^{2}$ was greater for the Fatigue performance group versus the Non-Fatigue group, indicating all model forms were better suited to predict new values in the Fatigue group. However, it must be noted that the relatively uniform performance of these subjects' results in a lower SST, and therefore decreases the calculated prediction $R^{2}$. If compared to the ordinary adjusted $R^{2}$ from the model development, the prediction $R^{2}$-values are of similar magnitude for the respective performance group. This trend indicates that the prediction fit for new observations will be on par with the original observation fits seen in the initial model development stages.

\subsubsection{MODEL GENERALIZABILITY}

Through the presented EMG-based regression analysis, two performance-specific models have been developed. These models fit their respective datasets and exhibit strong predictive capabilities, but are not robust in explaining response variability outside of their trained performance range. An attempt to find a General model through concatenation of the Fatigue and Non-Fatigue RPM profiles resulted in moderate predictive strength of RPM performance, once the 
model is trained. Fitting model coefficients to each subject resulted in highly fit models, but unique to the volunteer's data.

It is important to note that the muscles with strong performance correlations were the tibialis anterior (TA) and medial gastrocnemius (MG). The TA and MG are ankle plantar flexion and dorsiflexion actuators - not primary muscle generating muscles. A non-intuitive result, the importance of fine motor control muscles in dictating overall performance demands further investigation. Use of distal kinetic chain muscle activity and kinematics can potentially guide the development of performance metrics and augmentation devices across the body.

Relating these models to injury metrics can be facilitated through tracking of kinematics over the entire test duration. Reliance on the Vicon-to-OpenSim pipeline for osteokinematics significantly limits the ability to connect EMG regression models to observed kinematics and ITBS injury metrics in this study. Real-time pipelines for Vicon-to-OpenSim analysis will significantly aid future investigations in this area. Available kinematics of interest for iliotibial band pain syndrome could include hip adduction and hip internal rotation during peak knee loading. For patellofemoral pain syndrome, changes in rectus femoris and biceps femoris activity during increased hip adduction and hip internal rotation would be of interest. For lower back pain, increases in lumbar flexion and center of mass excursions (torso and pelvis) over the course of the cycle can be investigated. Literature kinematic trends with fatigue were observed in volunteer performance trends. Through this regression analysis, the isolation of several performance-correlated EMG parameters has been achieved. Developed EMG morphology metrics can be significantly affected by due to poor signal-to-noise quality of EMG measurements; pointing out the importance of EMG signal quality in translating these methods to application. Additional work is needed on the influence of fine motor control muscles on system-level performance and translating observed trends to muscle-based predictive models. 


\section{Chapter 4}

\section{IT-Band OpenSim Model}

\subsection{INTRODUCTION}

The current understanding of ITBS development mechanisms has shifted from a friction-based mechanism to a lateral femoral epicondyle (LFE) compression-based mechanism (Baker, Souza, and Fredericson 2011; Fairclough et al. 2007, 2006; Orchard 2007; Menard, Domalain, and Lacouture 2014). Soft tissue damage below the IT-band has been visualized through dissection and MRI-based studies, as recent as Summer 2017 (Godin et al. 2017; Flato et al. 2017; Sher et al. 2011; Seijas et al. 2016). While the location and symptoms of ITBS have been shown, the link between the hypothesized pathomechanics and the injury development has not been thoroughly studied. Limited by accessibility of the lateral knee during overuse injury development, experimental methods are often unable to reliable track longitudinal human joint changes without confounding factors (Ellis, Hing, and Reid 2007; Louw and Deary 2014). Use of computational musculoskeletal (MSK) models allows for two initial questions to be addressed:

1) What performance changes influence predicted LFE compression force?

2) Are known ITBS development metrics correlated with LFE compression force?

The aim of the final study in this thesis is to address these knowledge gaps through the development of an MSK model capable of LFE compression force prediction, and applying the model to laboratory-collected stationary bicycling data. The following sections present the methods and results of the conducted LFE compression force modeling with application to cycling injury (ITBS). Topics will include: OpenSim model development, biofidelity considerations, model evaluation, and model predictions. 


\subsubsection{MusCulOSKELETAL MuSCLES AND STRUCTURES OF INTEREST}

While testing included abdominal and back EMG, primary muscles and structures of interest were limited to the lower extremity. EMG signals from the rectus femoris (RF), biceps femoris (BF), medial gastrocnemius (MG), and tibialis anterior (TA) were measured bilaterally [Figure 4-1]. Due to sensor malfunction, left-leg BF data was excluded from analysis across all participants. In addition, when considering anatomical geometry in OpenSim model development, the attachment and interacting surfaces of the TFL were incorporated in the model. Regarding attachments of the TFL, rigid fixation of the TFL was defined at the proximal ASIS (origin), distal femur (Kaplan Fibers), and Gerdy's Tubercle (insertion). Contact surfaces along the length of the TFL included approximation of hip joint iliofemoral ligaments, greater trochanter of the femur, and lateral knee tissues [Figure 4-1].
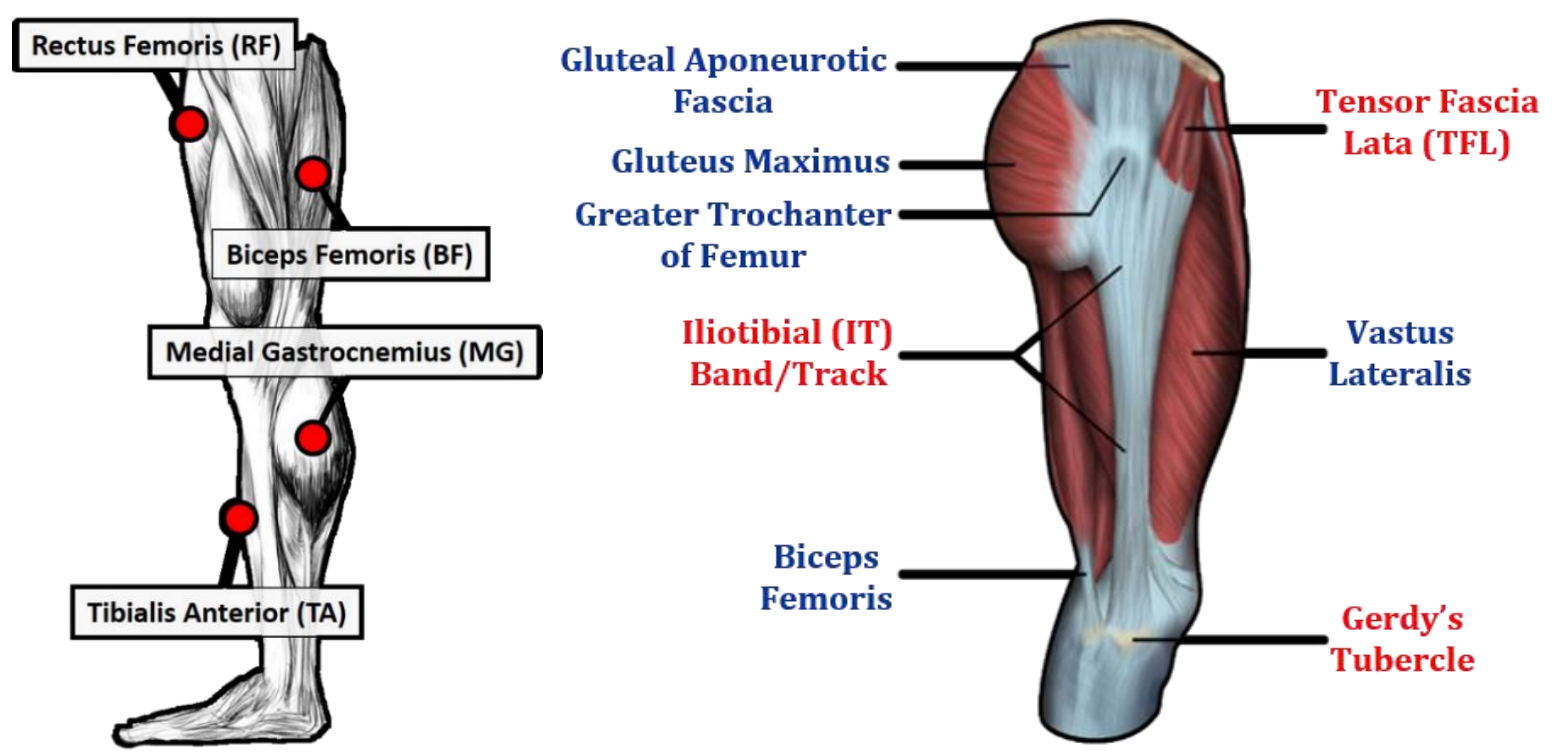

Figure 4-1: Anatomical Considerations for EMG Placement and OpenSim Model Development

\subsubsection{Muscle ACTIVATION MODEL IMPLEMENTATION}

Since force production in muscles is complex and nonlinear in nature, literature efforts to simplify and model muscle behavior are important. Through lumped-parameter modeling, numerical definitions of muscle dynamics can be adjusted across muscle types and anatomical considerations. The developed OpenSim model implements a form of the Thelen 2003 muscle model (Thelen 2003), which is a standard equilibrium model derived from the Hill model. A depiction of the muscle-tendon lumped-parameter model (Thelen 2003) is shown in Figure 4-2, with three model subunits: a contractile element, a parallel elastic element, and a series elastic element. 

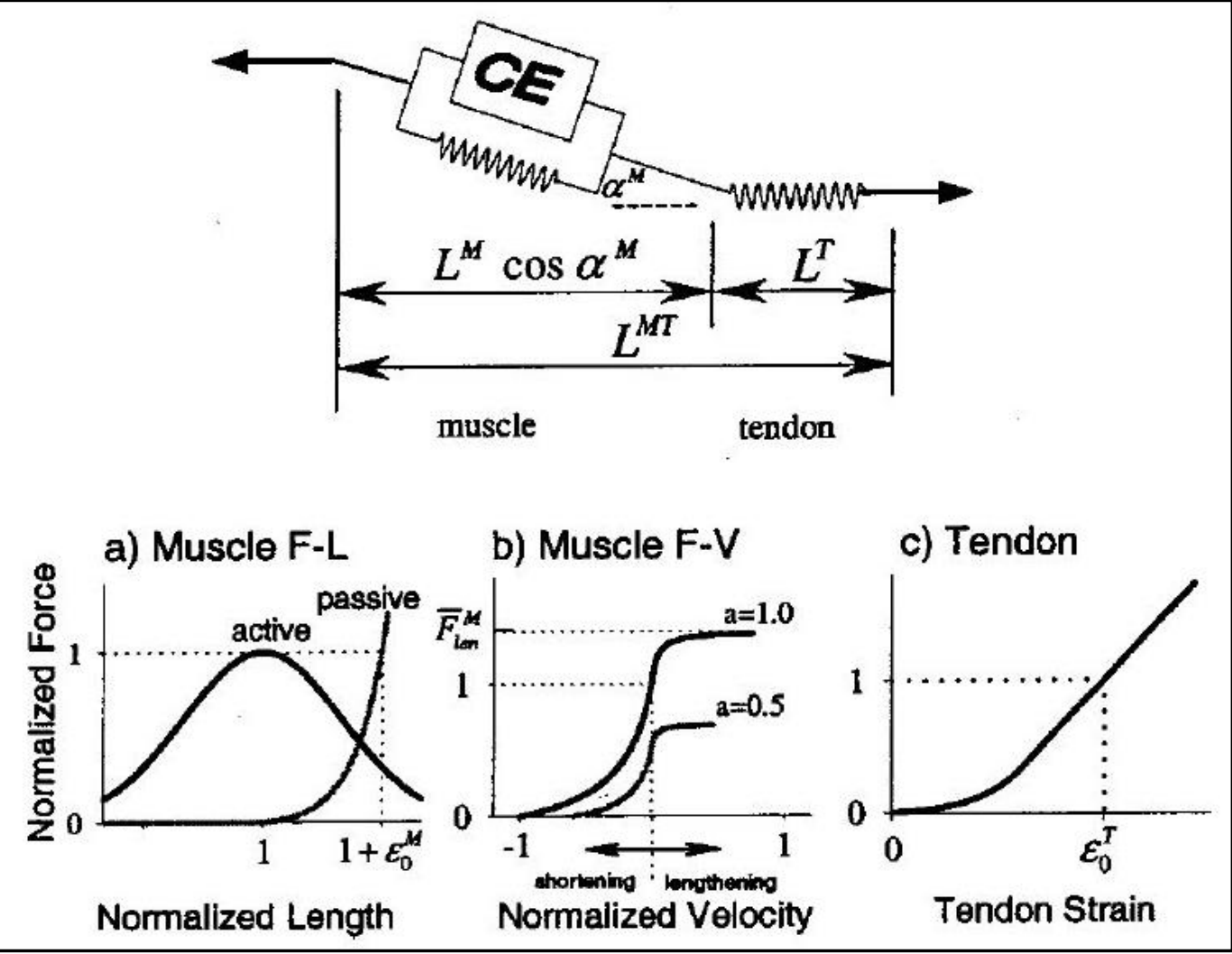

Figure 4-2: Lumped-Parameter Muscle Model from Thelen 2003. CE, L, F, and V denote contractile element, length, force, and velocity, respectively. Superscript M, T, and MT denote muscle, tendon, and muscle-tendon parameters, respectively. $\alpha^{\mathrm{M}}$ is used to denote the pennation angle of the muscle-tendon junction.

Muscle parameters used to define the Thelen 2003 element include: maximum isometric force, optimal muscle fiber length, tendon slack length, maximum contraction velocity, and pennation angle. For direct comparison of kinematic effects on LFE force prediction models, these metrics are kept consistent across subjects. When running other full-body analyses, muscle definition was allowed to scale with the subject size and weight characteristics. For implementation into OpenSim, developers applied the following modifications to remove discontinuities and numerical irregularities from the model definition:

1. Muscle and tendon units are massless

2. Smooth approach of activation dynamics equation to positive non-zero value.

3. Gaussian active-force-length curve (force always non-zero)

4. Singularity exists as tendon pennation angle approaches $90^{\circ}$.

5. Force-velocity curve invertible via extrapolation of muscle velocity.

6. Constraints: a) fiber length $>0.01 *$ optimal defined, and b) pennation $<$ max defined 


\subsection{CHAPTER OBJECTIVES}

The objectives of Chapter 4 on IT-band OpenSim modeling are as follows:

1) Improve OpenSim model biofidelity to enable estimation ITBS predictors. Baseline OpenSim model updates were guided by literature on cadaveric measurements of TFL-ITB path and attachments, and clinical observation of symptomatic tissues in ITBS patients.

2) Track ITBS predictors with fatigue-induced changes over cycling test and across system-level performance groups. Through similar analyses as done in Chapter 2, the updated OpenSim model is used to estimate LFE compression force and duration.

3) Provide insight on the implications of emergent error on ITBS development. Fatigueinduced changes in kinematics and ITBS predictors are analyzed to link cycling technique to overuse injury risk exposure and assess current clinical methods for evaluating risk.

\subsection{METHODS}

\subsubsection{OPENSim Model DEVElOPMENT}

To analyze subject-specific parameters not able to be measured experimentally, rigid body models, such as OpenSim, are utilized. OpenSim is an opensource multibody model developed by Stanford University. The model implements muscle actuators using idealized muscle models from literature. Starting with the OpenSim-provided "3DGaitModel 2354", markers were added to match the torso, leg, and lower extremity (Thelen et al. 2003). Additional markers were used for pedal and seat force application to the foot and pelvis, respectively. Head markers were excluded since the head and torso are modeled as a rigidly coupled body. Final marker placement used for scaling model geometry and kinematics to subject-specific experimental data are shown in Figure 4-3. 


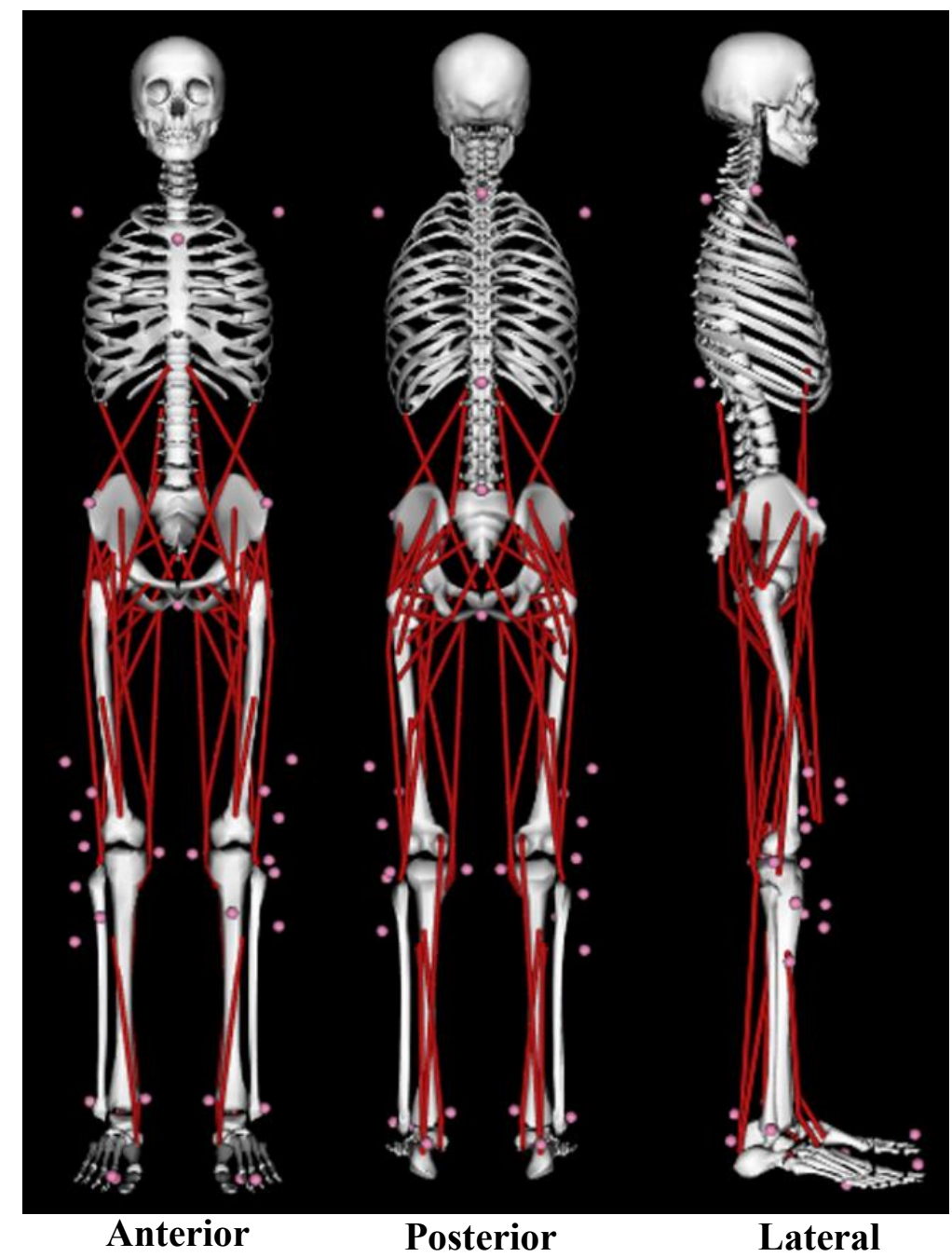

Figure 4-3: Final marker placement for OpenSim model. Orthogonal anterior (left), Posterior (center), and Lateral (right) views are presented.

Once the markers were placed, the base analyses through OpenSim (Scaling, Inverse Kinematics, Inverse Dynamics, and Computed Muscle Control) are possible with kinematic and boundary reaction forces applied. However, for more accurate biofidelic predictions of muscle forces and muscle-bone forces, base OpenSim models have a major hurdle: muscle paths are often rigidly fixed to the bone. Physiologically, this leads to incorrect muscle lengthening and shortening during motion, which plays a significant role in resultant muscle force for a specific activation level (Thelen 2003). In this study, ITBS risk factors are of interest, all of which rely on accurate modeling of the Tensor Facia Lata (TFL) path and estimation of lateral femoral epicondyle (LFE) compression force. Figure 4-4 provides an overview of the iterative OpenSim model development process and details are below. 


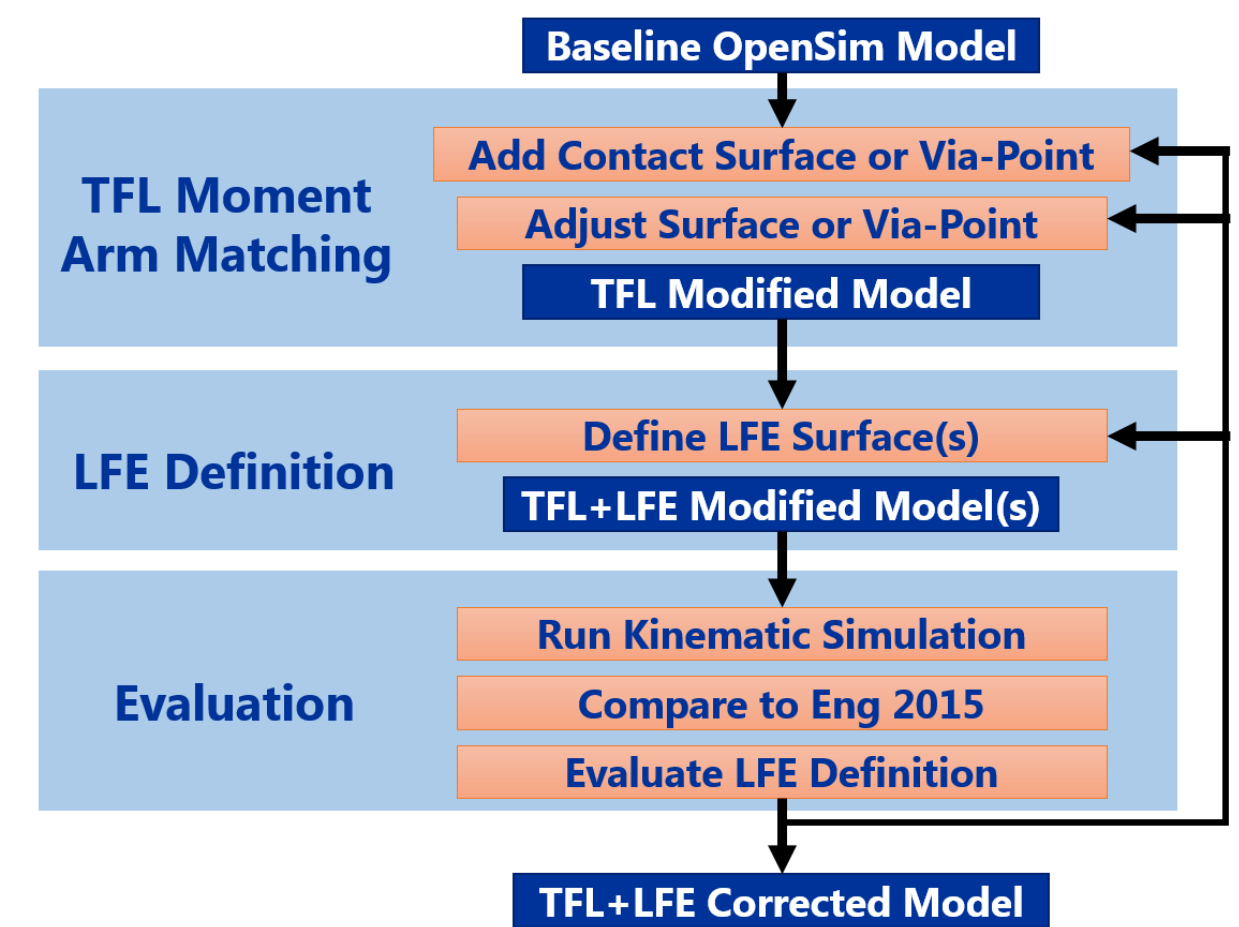

Figure 4-4: Overview of OpenSim Model Development Process

One approach for improving model TFL biofidelity is matching model TFL moment arm data with experimentally measured TFL moment arm over a range of hip and knee motions. A 2015 study measured TFL and Glute moment arms from 10 cadaveric legs (Eng et al. 2015). The resultant moment arm corridors [Figure 4-5] were used to adjust OpenSim TFL path. 

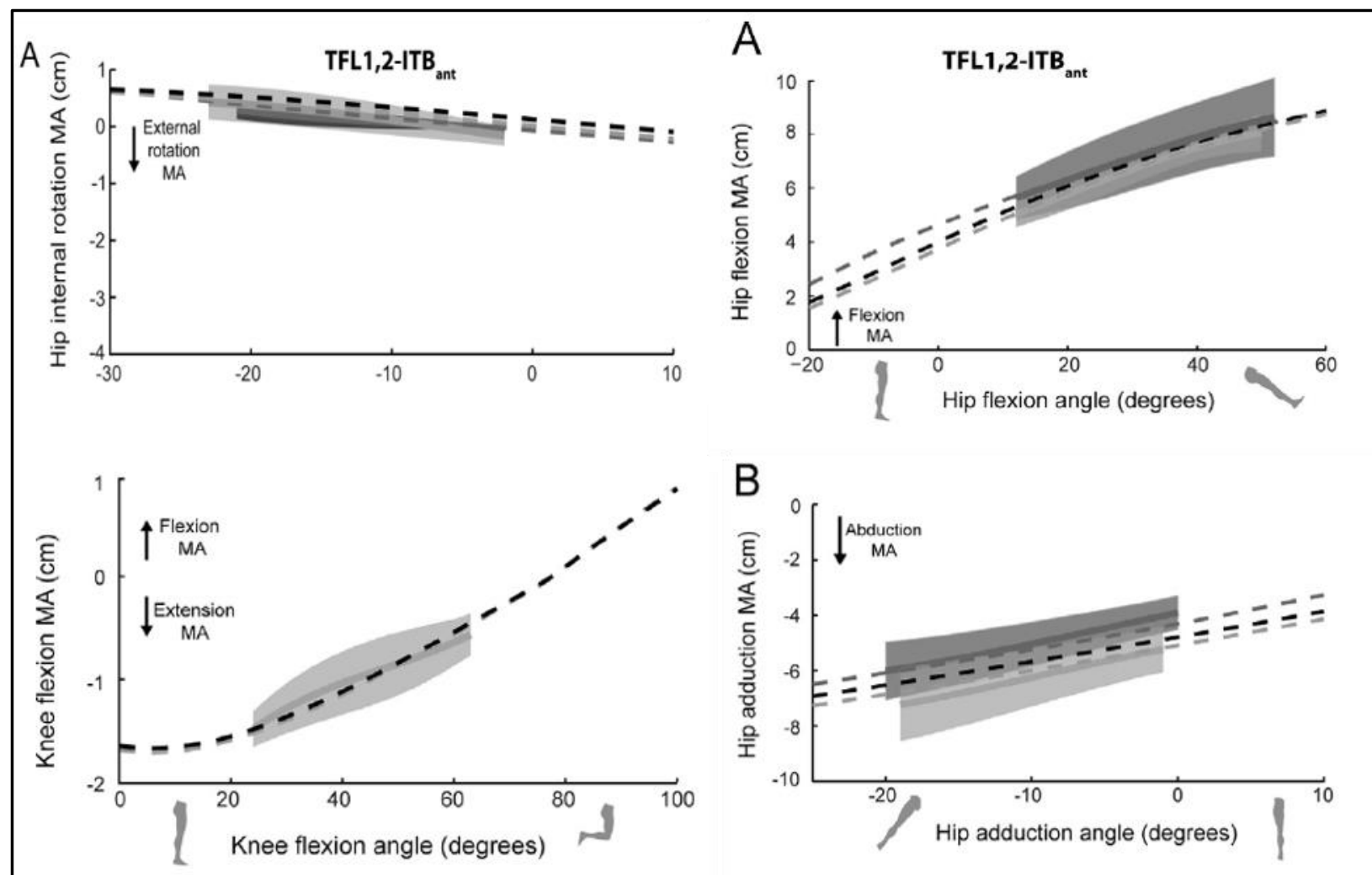

Figure 4-5: Moment arm corridors from Eng et al. (2015) used for improving biofidelity of OpenSim model predictions of TFL forces

A combination of ellipsoid and torus wrapping surfaces were implemented, with consideration for underlying bony and soft tissue structures [Figure 4-6]. Notable structures in the model include ligamentous tissues of the hip joint capsule, Greater Trochanter of the Femur, femoral shaft, and lateral femoral epicondyle. During model development, a single leg model was used to accelerate the trial-and-error process of wrap surface adjustments. 


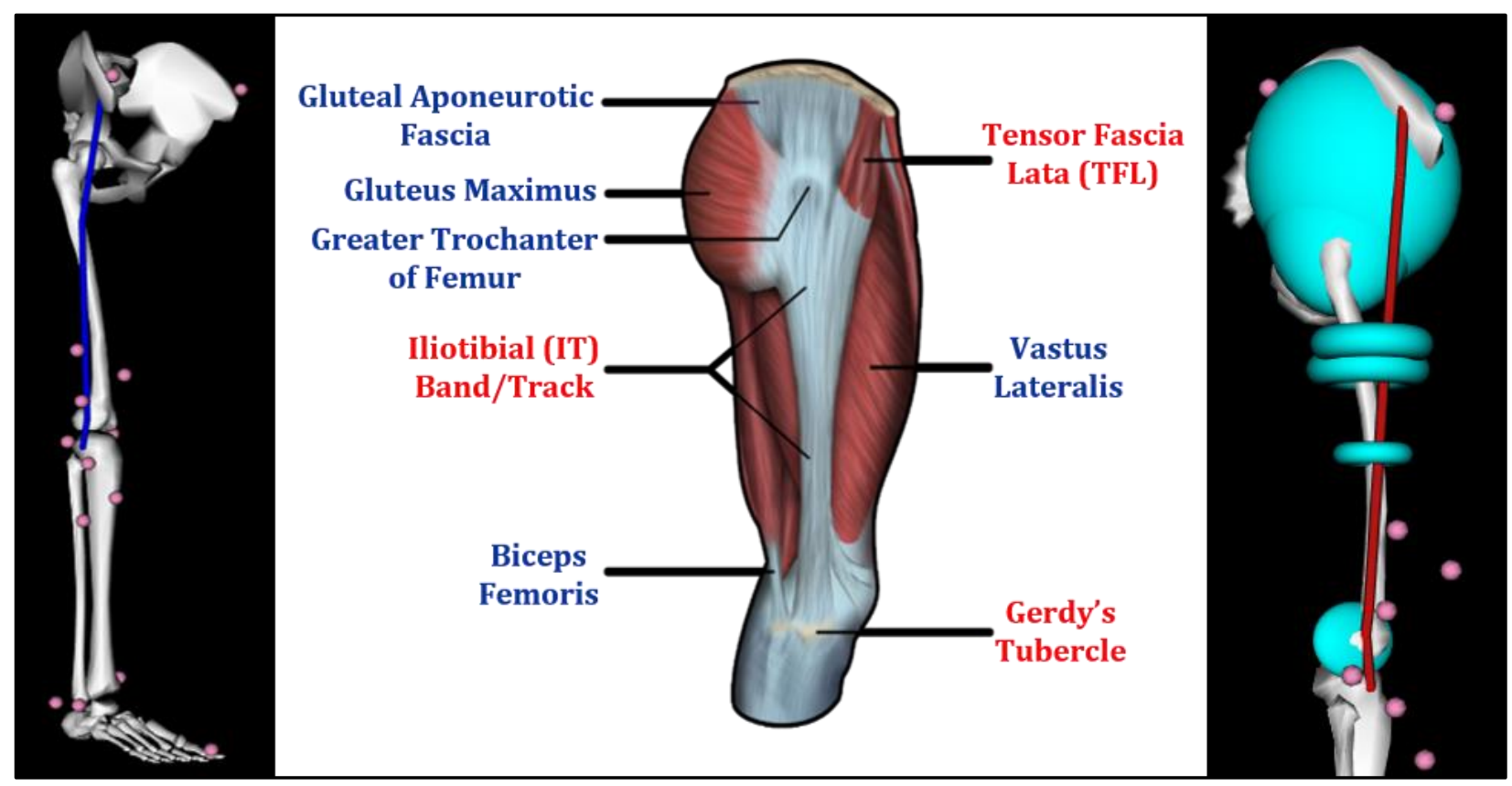

Figure 4-6: Implementation of wrap surfaces for TFL moment arm correction. Base model (left), anatomy of lower extremity (center) and final TFL wrap surface model (right) are presented.

After establishing a moment-arm-matched TFL model, the next step was to develop a method for estimating TFL-LFE compression force. To make use of existing OpenSim model components, an additional zero-mass "LFE" body was defined and rigidly bound to the femur. Two methods were investigated: implicit and explicit modeling of the LFE contact surface. Both methods incorporate wrap ellipsoids in the "LFE" body coordinate system, thereby allowing compression force to be estimated as the "LFE-femur" joint forces. Implicit uses a single wrap ellipsoid in the "LFE"-body frame to model the LFE and lateral knee structure. Explicit modeling, however, uses a wrap ellipsoid to model lateral knee structure in the "femur"-body frame, and a second wrap ellipsoid to model the LFE in the "LFE"-body frame. Size and placement of the explicit LFE surface was estimated using inflammation regions (soft-tissue damage) observed in recent MRI imaging of ITBS symptomatic knees [Figure 4-7] (Flato et al. 2017). Images of the implicit and explicit LFE models are presented, below [Figure 4-8]. 

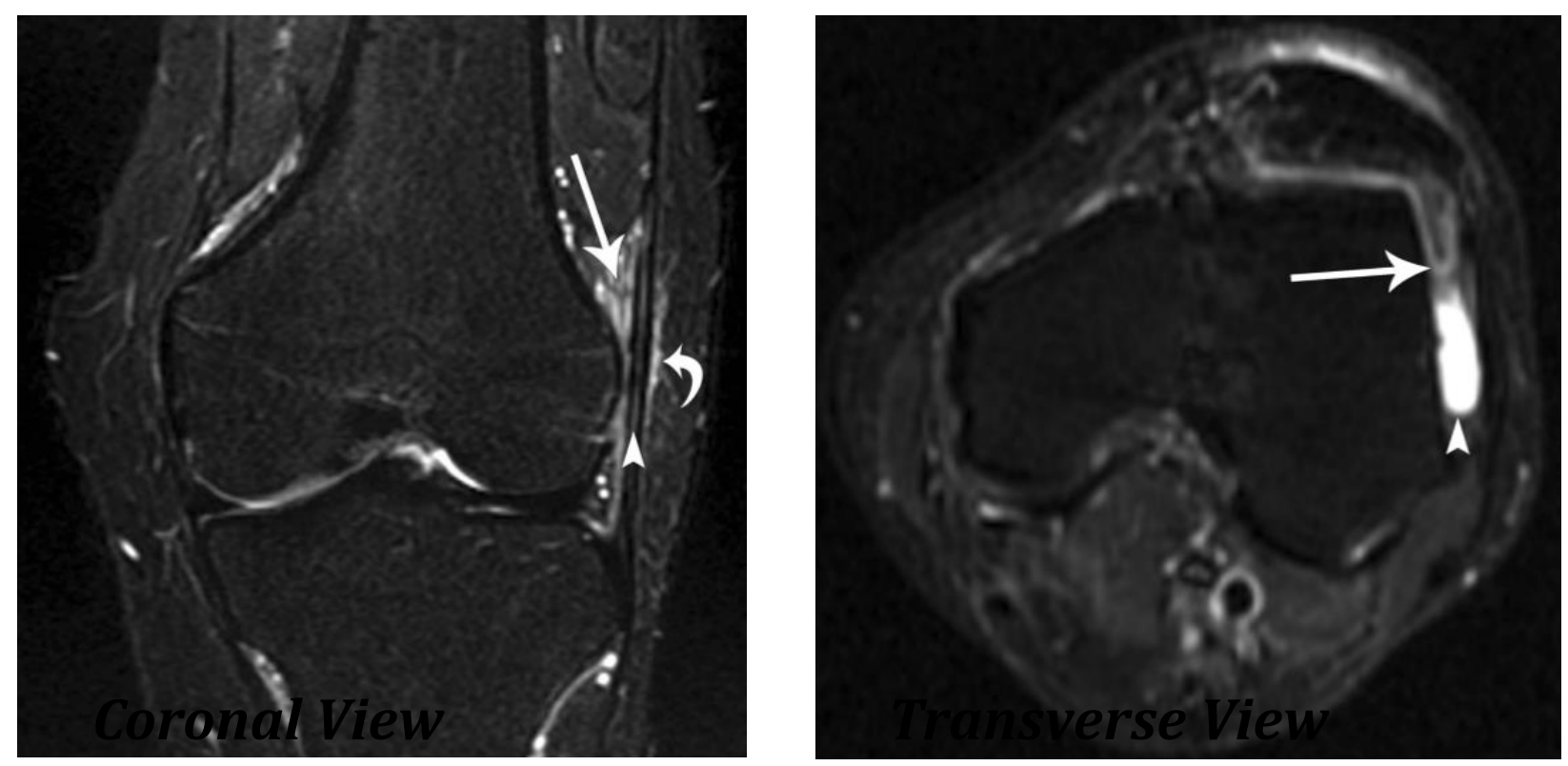

Figure 4-7: MRI images from Flato et al. (2017) of symptomatic knees from ITBS patients. Frontal view (left) showing inflammation (arrows) of tissue surrounding IT band (arrowhead). Transverse view (right) showing edema-like fluid accumulation (arrowhead) and inflammation (arrow) medial to IT band.
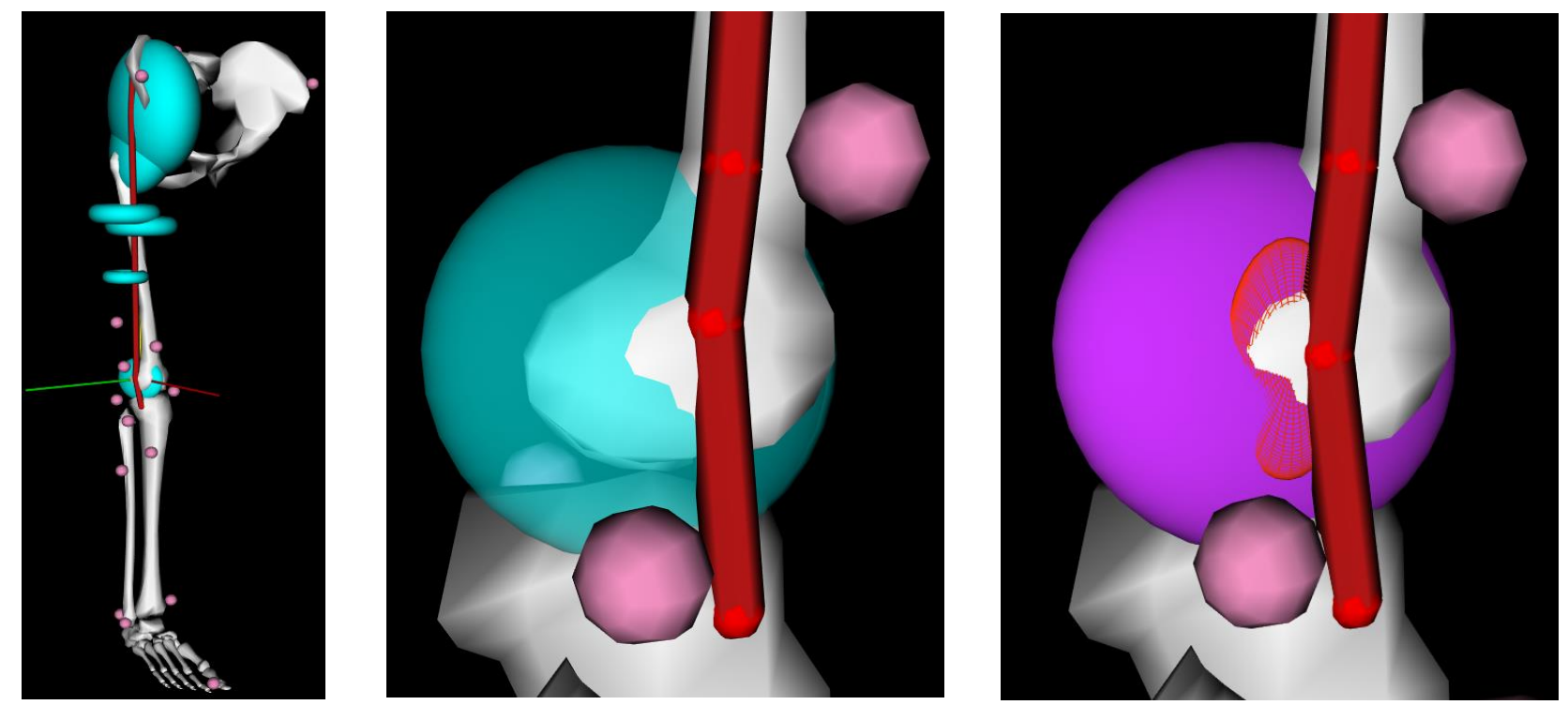

Figure 4-8: Models considered for LFE contact surface definition. Oblique view of final TFL model (left). Lateral view of implicit LFE model (center), with lateral knee represented by blue ellipsoid. Lateral view of explicit LFE model (right), with LFE and lateral knee represented by red and purple ellipsoids, respectively. 


\subsubsection{OPENSIM ANALYSIS PIPELINES}

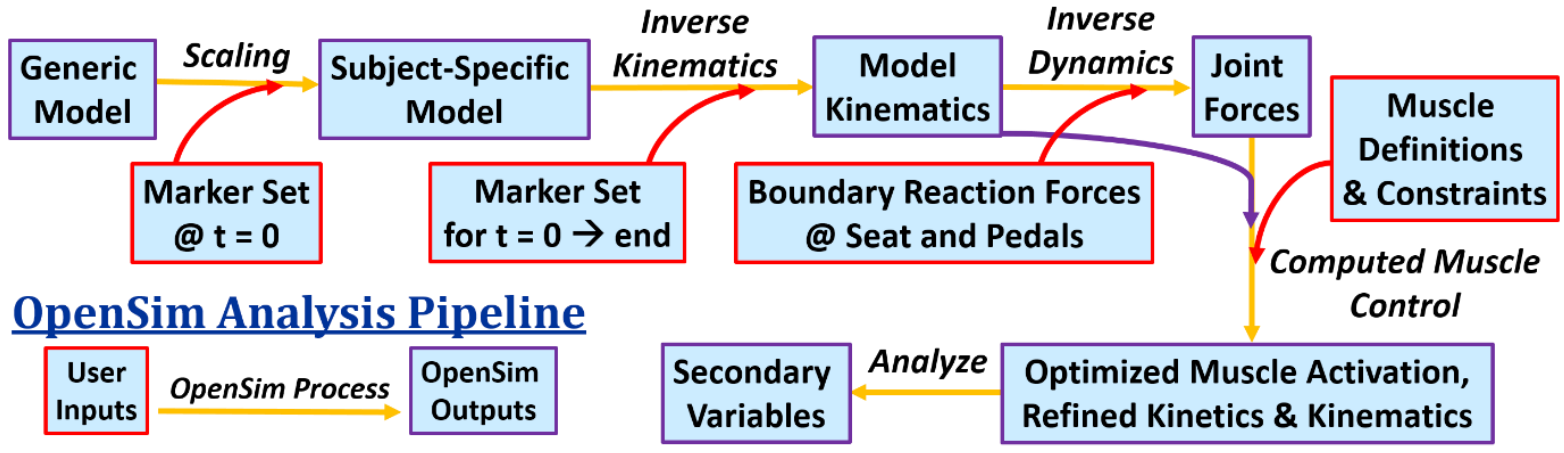

Figure 4-9: OpenSim Analysis Pipeline. Analytical tools in OpenSim are represented by orange arrows. User inputs, and computational outputs are outlined in red and purple, respectively.

\section{Scaling}

The scaling function in OpenSim allows the user to fit the skeleton model to a specific subject. In other words, using the marker set data obtained from Vicon and defined through Nexus, OpenSim can calculate the length of the bones and general proportions of a subject's entire body. For example, specific measurements of the distance between the markers found on the right and left ASIS are used to scale the pelvis.

\section{Inverse Kinematics}

The inverse kinematics function allows the user to take the scaled model, along with the defined Vicon marker data from Nexus, to recreate the motion exhibited by the subject. With this motion recreated, OpenSim is able to calculate the kinematics of the subject during the trial. Data obtained from inverse kinematics are values such as joint angles. These joint angles allow for analysis of subject kinematic patterns such as compensation or improper technique. Inverse kinematics are an essential basis for calculating inverse dynamics and computed muscle control.

\section{Inverse Dynamics}

The inverse dynamics function allows the user to take the scaled model, the inverse kinematic data, and the kinetic forces, recorded during the trial, and combine them to back calculate the forces throughout the body. In other words, OpenSim takes the motion data and combines it with the reaction forces to understand where those forces are coming from within specific bodies in the 
model. The data produced by the inverse dynamics is the moments or joint forces that align with the kinematics to create the reaction forces that observed.

\section{Computed Muscle Control}

The computed muscle control (CMC) function allows the user to take the scaled model, the inverse kinematics data, the inverse dynamics data, and the recorded kinetic forces to calculate the subject's muscle activation. When played back through OpenSim, the user can see what muscles are being activated at what times in the trial via highlighting the muscles in blue during activation. This data is useful and can be studying along with the kinematic data to confirm compensation patterns that occur. Also, this muscle activation data can also be collected and compared to data obtained by attaching EMGs. The CMC function produces every aspect of the muscle, including: activation, lengths, and movement.

\section{Analyze}

The Analyze function allows the user to collect all the data that can be obtained from inverse kinematics, inverse dynamics, and CMC. When you run these three functions, OpenSim provides the essential information that is needed to run the next step of the tool pipeline. To gain additional information from the model (i.e. internal joint loads, muscle activation dynamics), Analyze must be run after CMC. Analyze was used to estimate TFL activation properties and LFE compression forces in the model. 


\subsubsection{Model Evaluation \& APPLiCATION}

After model development, the two models were evaluated for TFL moment arm biofidelity and force prediction signal quality. Three main model components were evaluated after modification:

1) TFL moment arm over hip and knee ROM,

2) TFL force response surface over hip and knee ROM

3) Compressive force predictions at the LFE.

\section{TFL Moment Arm Evaluation}

Through trial-and-error, wrap surfaces are adjusted on the base-OpenSim model to match the TFL moment arm corridors from Eng et al. 2015. To evaluate model TFL moment arms, OpenSim's analyze tool run with user-defined kinematics. As done in Eng 2015, the starting position for any joint kinematic was a "standing pose" of $0^{\circ}$ hip flexion, $0^{\circ}$ hip adduction, $+5^{\circ}$ hip internal rotation, and $0^{\circ}$ knee flexion. From this initial pose, a combination of Python and MATLAB scripts were used to run the model through a single-DOF kinematic at each of the joints listed. Coupled-DOF, static position, and activation cycling models were also run [Table 4-1]. Max TFL force was set to $100 \mathrm{~N}$ at $100 \%$ "activation" for all evaluation simulations to normalize force predictions across subject muscle strengths and allow comparisons of kinematic and activation level effects on compression force. The predicted TFL moment arm were fit to Eng 2015 corridors.

Table 4-1: Simulations Run for Evaluation of Updated TFL Model

\begin{tabular}{|c|c|c|c|c|c|c|}
\hline & \multicolumn{4}{|c|}{ Joint Kinematic ROM (Min, Max) } & \multirow{2}{*}{$\begin{array}{c}\text { TFL } \\
\text { Activation } \\
(0 \text { to }+1)\end{array}$} & \multirow{2}{*}{$\begin{array}{c}\text { TFL } \\
\text { Fiber } \\
\text { Length } \\
(\mathrm{mm})\end{array}$} \\
\hline Model & Knee Ext. & Hip Flex & Hip Add. & $\begin{array}{l}\text { Hip Int. } \\
\text { Rot. }\end{array}$ & & \\
\hline Knee Ext & $-120^{\circ},+10^{\circ}$ & $0^{\circ}$ & $0^{\circ}$ & $+5^{\circ}$ & +1 & +7 \\
\hline Hip Flex & $0^{\circ}$ & $-5^{\circ},+80^{\circ}$ & $0^{\circ}$ & $+5^{\circ}$ & +1 & +7 \\
\hline Hip Add & $0^{\circ}$ & $0^{\circ}$ & $-20^{\circ}, 15^{\circ}$ & $+5^{\circ}$ & +1 & +7 \\
\hline Hip Rot & $0^{\circ}$ & $0^{\circ}$ & $0^{\circ}$ & $-15^{\circ},+10^{\circ}$ & +1 & +7 \\
\hline Knee Ext + Hip Flex & $-120^{\circ},+10^{\circ}$ & $-5^{\circ},+80^{\circ}$ & $0^{\circ}$ & $+5^{\circ}$ & +1 & +7 \\
\hline Knee Ext + Hip Add & $-120^{\circ},+10^{\circ}$ & $0^{\circ}$ & $-20^{\circ}, 15^{\circ}$ & $+5^{\circ}$ & +1 & +7 \\
\hline Knee Ext + Hip Rot & $-120^{\circ},+10^{\circ}$ & $0^{\circ}$ & $0^{\circ}$ & $-15^{\circ},+10^{\circ}$ & +1 & +7 \\
\hline Hip Flex + Hip Add & $0^{\circ}$ & $-5^{\circ},+80^{\circ}$ & $-20^{\circ}, 15^{\circ}$ & $+5^{\circ}$ & +1 & +7 \\
\hline Hip Flex + Hip Rot & $0^{\circ}$ & $-5^{\circ},+80^{\circ}$ & $0^{\circ}$ & $-15^{\circ},+10^{\circ}$ & +1 & +7 \\
\hline Hip Add + Hip Rot & $0^{\circ}$ & $0^{\circ}$ & $-20^{\circ}, 15^{\circ}$ & $-15^{\circ},+10^{\circ}$ & +1 & +7 \\
\hline Static Pose & $0^{\circ}$ & $0^{\circ}$ & $0^{\circ}$ & $+5^{\circ}$ & +1 & +7 \\
\hline $\begin{array}{c}\text { Static Pose }+ \\
\text { Activation Cycle }\end{array}$ & $0^{\circ}$ & $0^{\circ}$ & $0^{\circ}$ & $+5^{\circ}$ & $0,+1$ & $\begin{array}{c}+7 \\
+100\end{array}$ \\
\hline
\end{tabular}


Each simulation was run with a joint angle increment $(\Delta \theta)$ of 1 degree, with time step (dt) of 0.01 second per angle increment. Force outputs were found with static optimization at each time step as to remove muscle activation dynamics in the evaluation simulations.

\section{LFE Force Evaluation}

Two models of the lateral femoral epicondyle were developed. To evaluate these models, a constant TFL muscle "activation effort" model with each LFE setup was run through the full knee flexion-extension range of motion. Constant muscle "effort" was defined as constant muscle activation level, and constant fiber length, thereby allowing muscle lengthening over the range of motion to effect TFL actuation force. Reaction forces were measured at the weld-joint defined between the LFE-body and the femur-body. To remove the effect of muscle lengthening, LFE forces were also normalized by TFL force, resulting in the proportion of TFL force translated to each component of LFE reaction loading.

\subsection{RESULTS}

\subsubsection{ITB Model Evaluation}

Three main model components were evaluated prior to application to subject data:

1) TFL moment arms,

2) TFL force response over ROM, and

3) LFE force prediction.

While developing the TFL model, priority was made to match Eng 2015's model moment arm data. If adjustments in the wrap surfaces led to moment arm responses incompatible with the Eng model, the adjustments were focused on maximizing corridor fit. Deviations from Eng 2015 were more accepted outside of the corridor data and outside of the stationary cycling range of motion. Graphs used to evaluate fit of the developed models with Eng 2015 are presented [Figure 4-10]. 


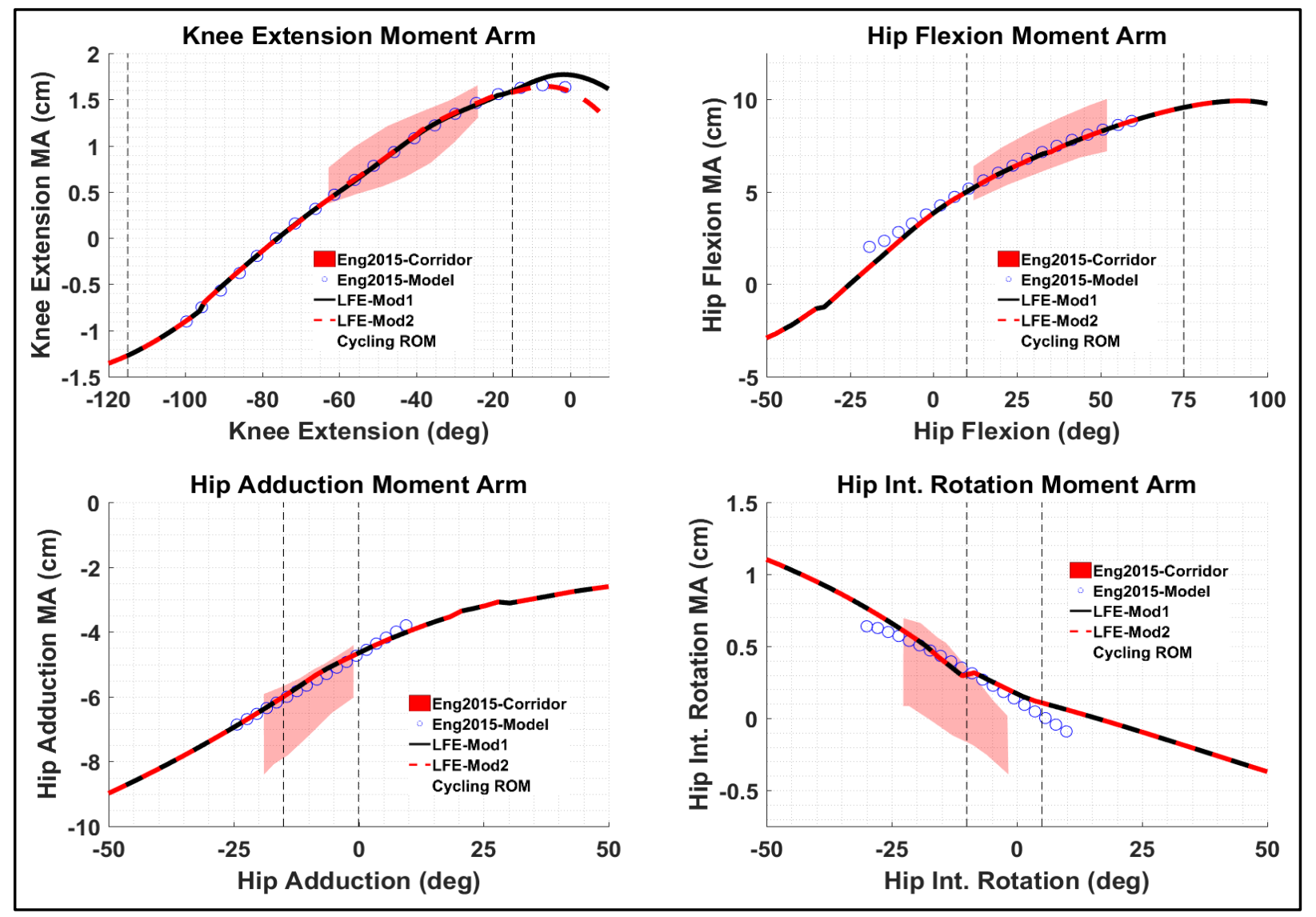

Figure 4-10: TFL Moment Arm Corridor Fitting. Eng 2015 corridors and Eng 2015 model data are presented by the red shaded area, and the blue dots, respectively. Moment arm results from Model 1 and Model 2 of the LFE from the current investigation are presented in black and red lines, respectively. The thin dashed black line indicates the approximate joint range of motion observed in stationary cycling.

Model adjustment iterations were concluded when the TFL moment arm converged to approximately match Eng 2015's cadaveric corridors and/or that of their MSK model. To see how the muscle model responds to activation and fiber length variation, a static pose simulation with dynamic TFL activation and fiber length was run. A force-limited $100 \mathrm{~N}$ muscle model was used. The imposed activation and fiber length cycling for the dynamic muscle activation simulation and the force response of the TFL's Thelen 2003 muscle model is presented [Figure 4-11]. 


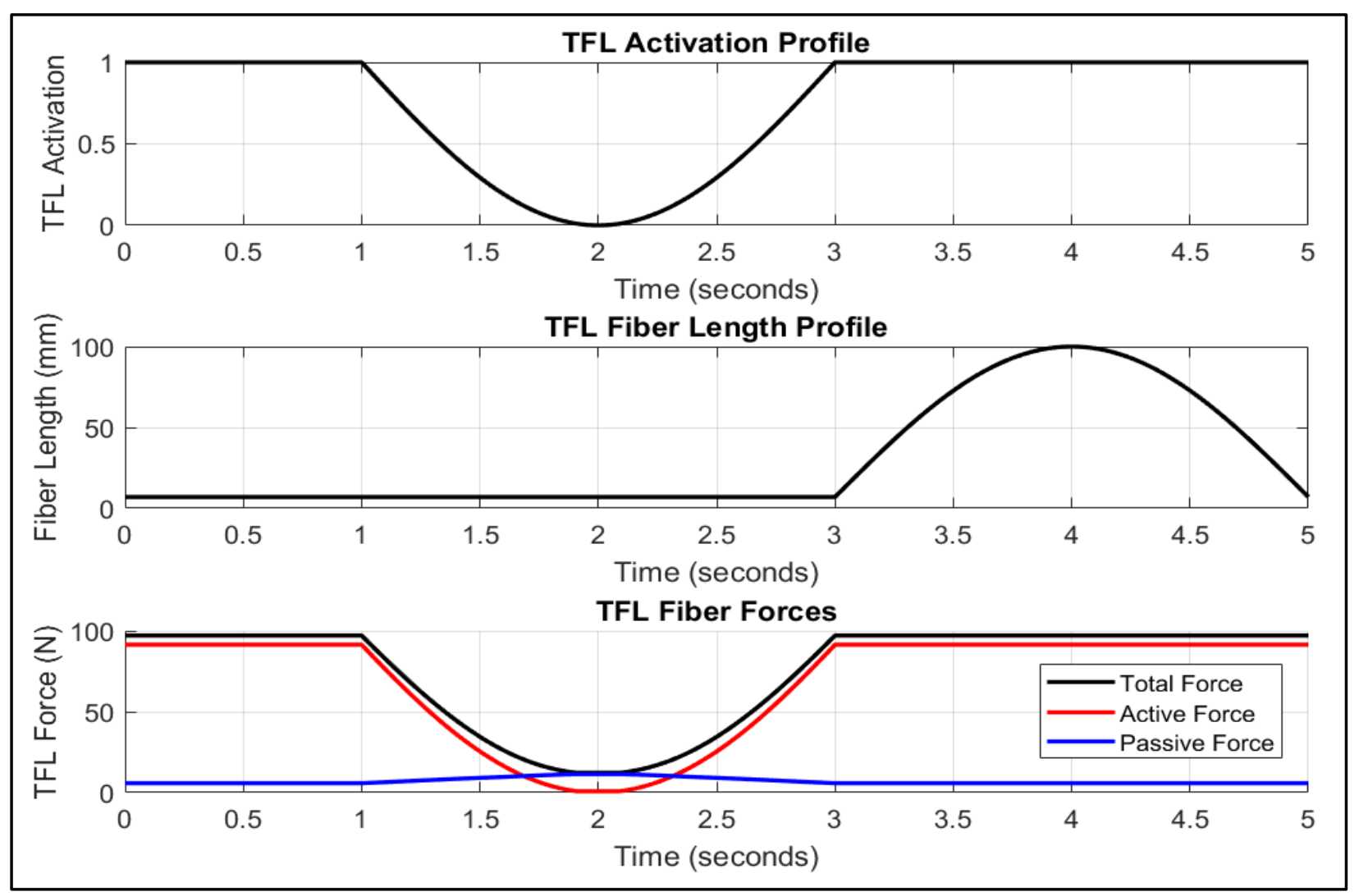

Figure 4-11: Static Pose Dynamic Activation Simulation to Evaluate TFL Muscle Model. Applied activation (top), fiber-length (middle), and TFL force output (bottom). Muscle force is seen to vary directly with activation level, with minimal change due to muscle fiber-length variation.

The model was then run through a series of single and double DOF simulations to measure how a constant-activation and constant-fiber-length TFL's force changes over the bicycling ROM. Starting from the standing static pose, the changes in TFL force for the single DOF simulations are presented below [Figure 4-12]. 

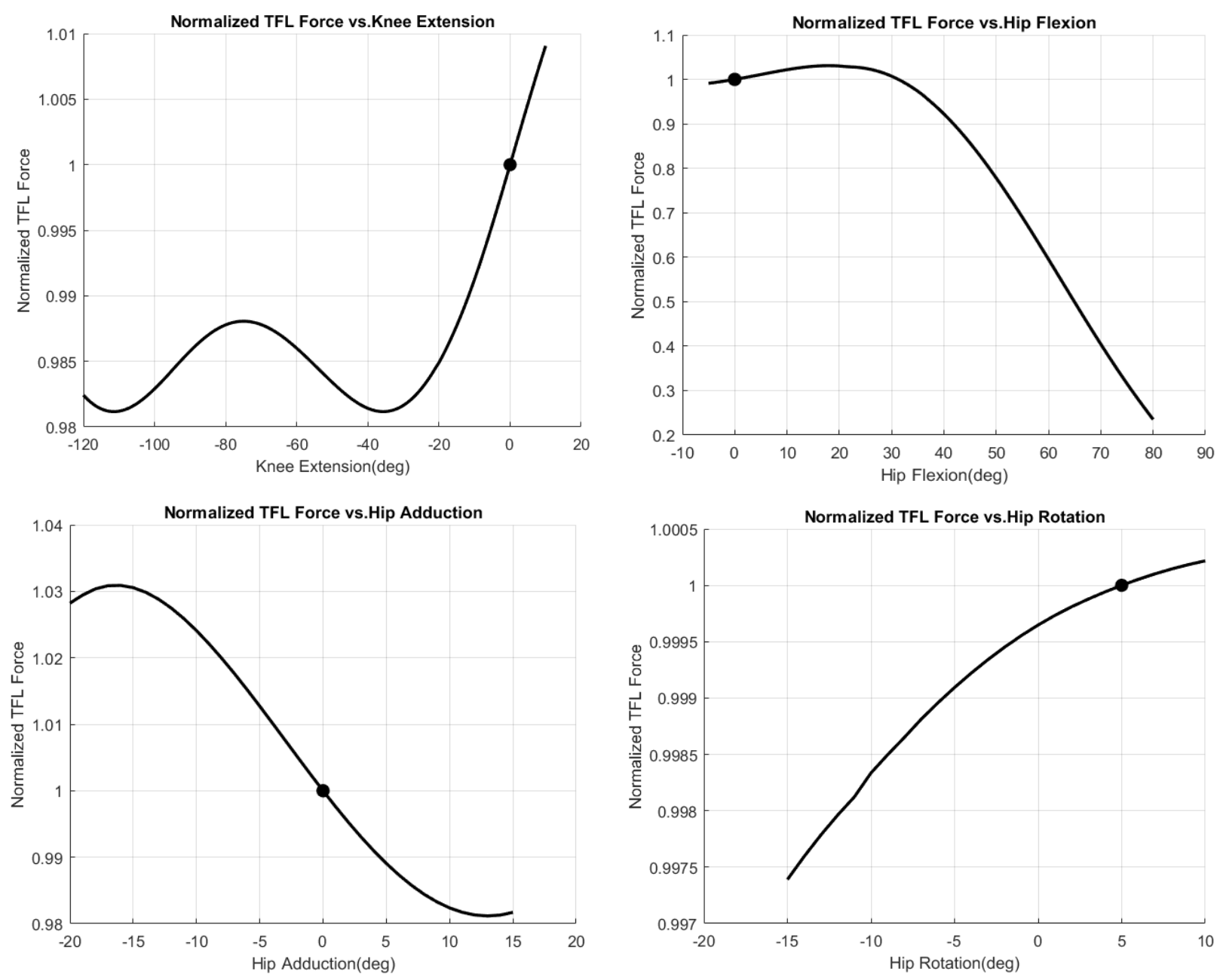

Figure 4-12: TFL Force Response to Single DOF Hip and Knee Kinematics under Constant Activation (100\%) and Constant Fiber Length $(7 \mathrm{~mm})$. Muscle length could vary along joint angle variations in the model. Forces are normalized to predicted TFL force at a standing static pose.

TFL force does not show significant force change over the observed ranges of knee extension, hip adduction, and hip rotation. The maximum TFL change of these three DOFs is a $3 \%$ force increase when moving to a more abducted hip position from standing. Hip flexion contributed significantly to TFL force, reducing force by more than $70 \%$ when flexed at $80^{\circ}$ from standing $\left(0^{\circ}\right)$.

Hip and knee joint kinematic ranges were then coupled to generate a series of two-DOF simulations [Figure 4-13]. Through these models, the effect of concurrent osteokinematics on muscle lengthening and TFL force generation can be visualized. 


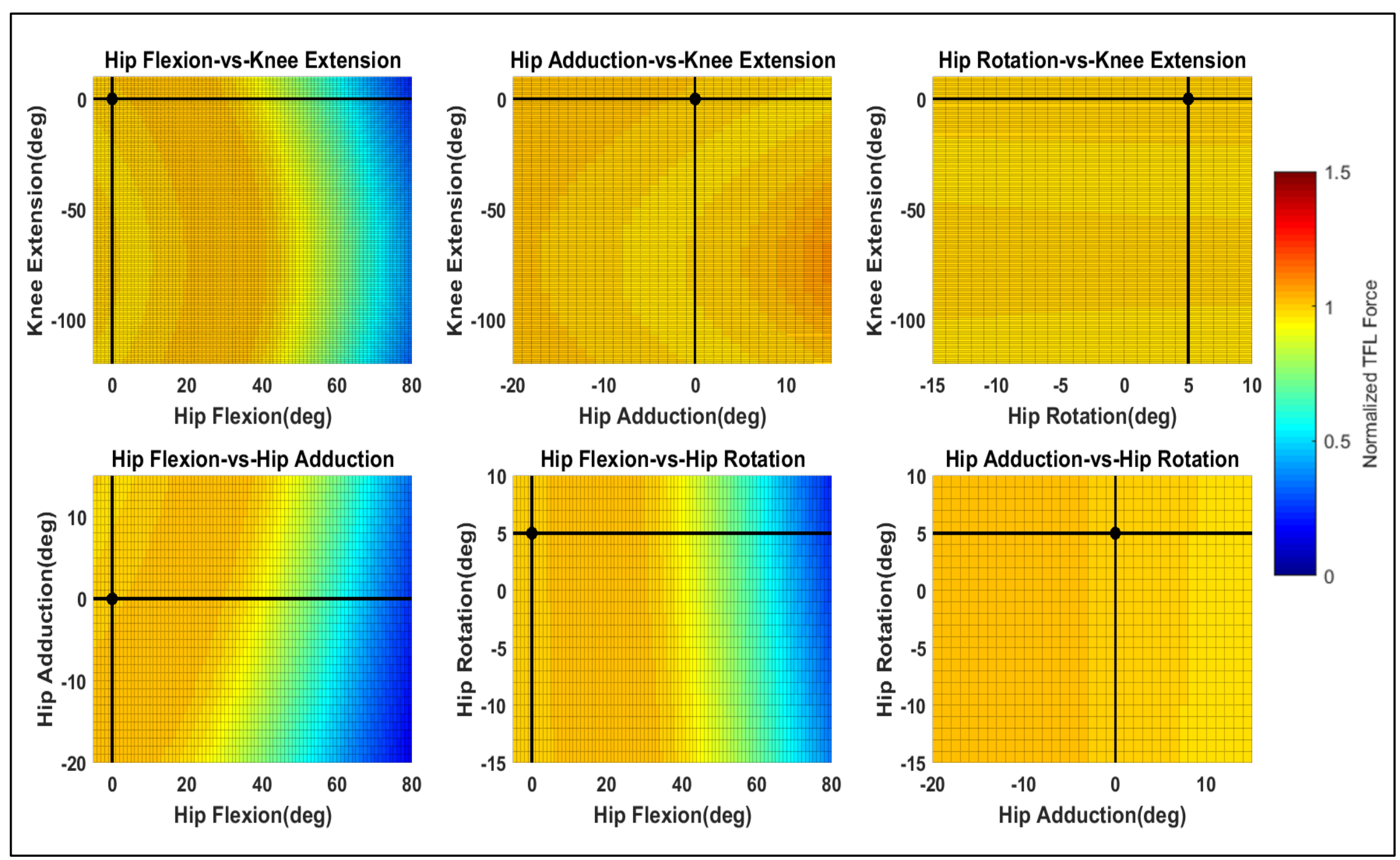

Figure 4-13: Normalized 2-DOF TFL Force Response Surface. Black dots indicate neutral standing position from Eng 2015. Black lines indicate ROM along which 1-DOF plots were generated. Forces are normalized to predicted TFL force at a standing static pose. 
LFE compressive force prediction was developed through both implicit (Model 1) and explicit (Model 2) of the lateral femoral epicondyle. The predicted LFE force components (normalized by TFL force) are presented below [Figure 4-14]. Through explicit modeling of the LFE, force outputs had discontinuities as the TFL transitioned from contacting the lateral knee surface to the LFE. Such discontinuities were easily removed through smoothing of the predicted force profile.
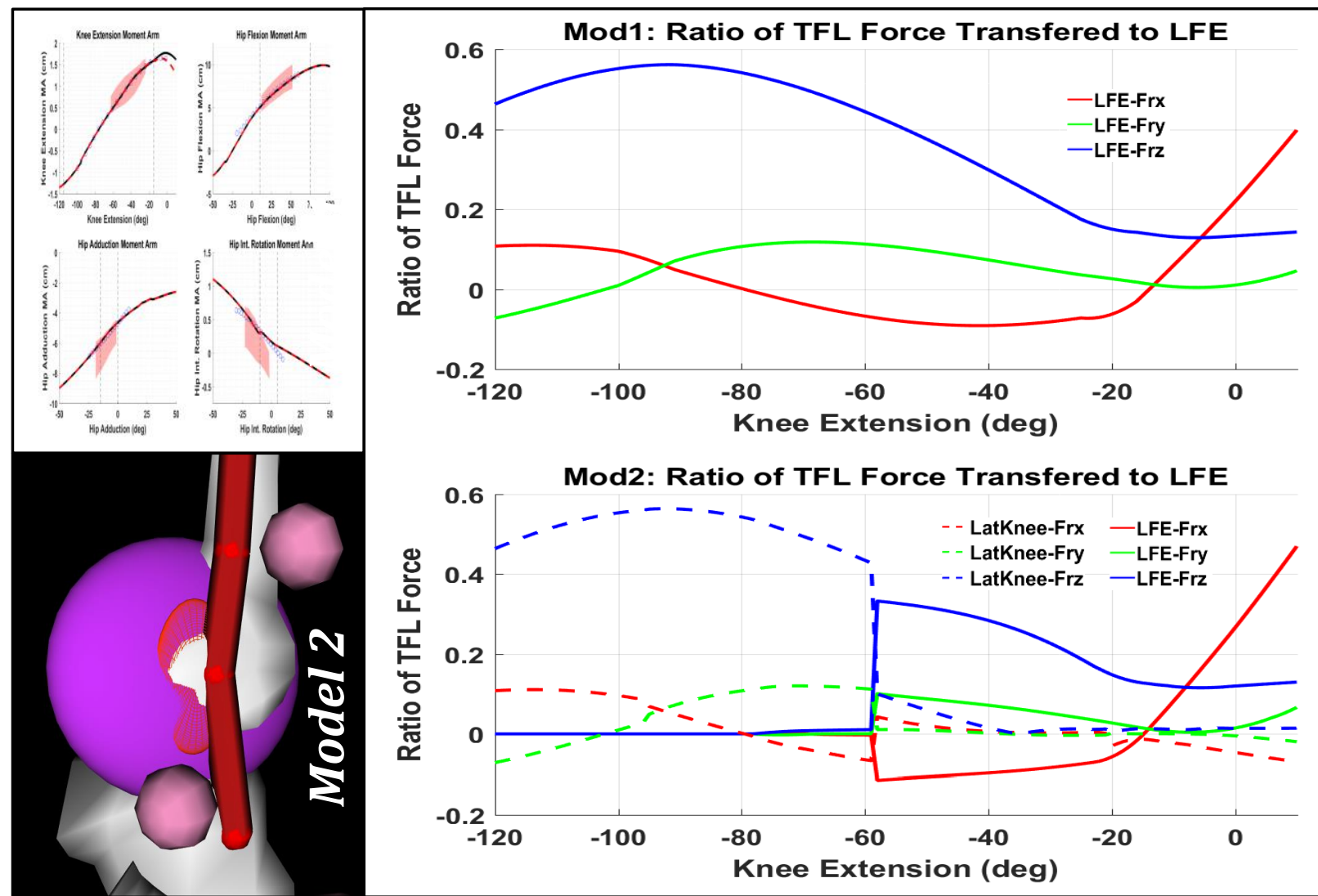

Figure 4-14: LFE Model Force Predictions. Model 1 (top) implicitly models the LFE, while Model 2 (bottom) explicitly model the LFE. Increased biofidelity of Model 2 is traded off by increased numerical instability at the muscle-surface contact transition points.

While LFE Model 2 is more biofidelic, wrap surface transitions in this model increased computational time and introduced numerical instability. Instead of implementing Model 2 in the OpenSim Analyze tool for LFE compression force estimation, LFE Model 2 was converted to a look-up table. LFE Model 1 was used in OpenSim Analyze to maintain TFL moment arm changes with knee flexion while removing numerical instability sources. MATLAB scripts were used postsimulation to utilize Model 2 interpolation functions to predict LFE compression and shear forces. 


\subsubsection{ITB MODEL APPLICATION}

Following evaluation of the TFL wrapping surfaces and the LFE modeling method, the developed model components were introduced bilaterally into the subject-specific OpenSim models [Figure 4-15].

Prior to applying subject data to the updated full-body model, the single leg model was run through a series of 2-DOF kinematics to evaluate TFL lengthening effects on muscle force. For the 4-DOF in the simplified 1-leg model, a total of 6 unique combinations were tested [Table 4-1]. These DOF combinations included:

- Knee Flexion + Hip Flexion

- Knee Flexion + Hip Adduction

- Knee Flexion + Hip Internal Rotation

- Hip Flexion + Hip Adduction

- Hip Flexion + Hip Internal Rotation

- Hip Adduction + Hip Internal Rotation

To evaluate TFL lengthening, a constant "activation effort" muscle model was implemented. Resultant forces were normalized by the TFL force generated by standing with $100 \%$ activation effort $(\sim 100 \mathrm{~N}$ of fiber force).

Plotting these results provided response surfaces by which the effect of each joint DOF on total TFL force can be seen [Figure 4-16a and Figure 4-16b]. Responses surfaces were overlaid with average bilateral kinematics at the hip and knee for three stages of the endurance ride: pre-fatigue, intermediate stage, and fatigue/failure.

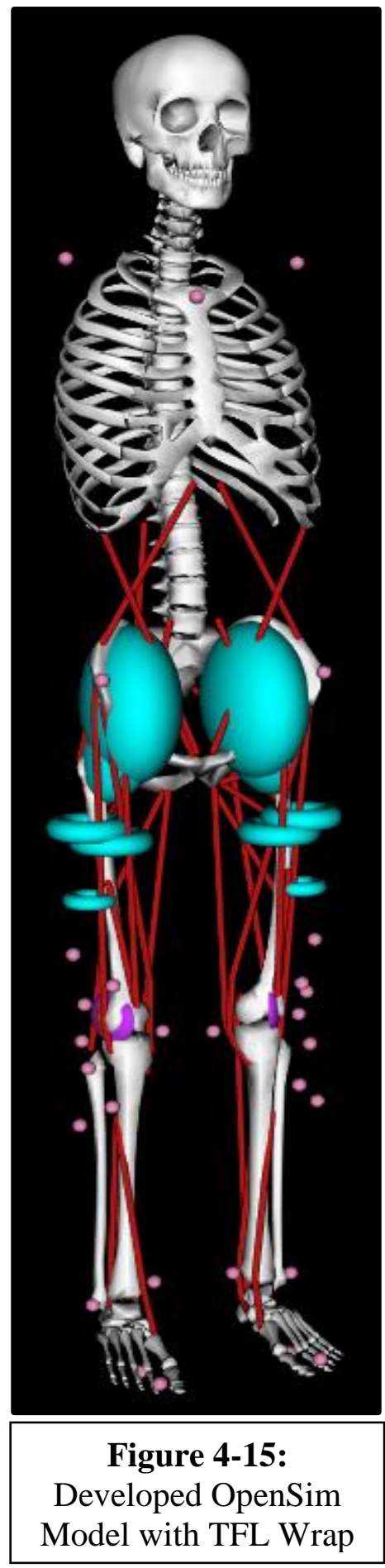

Average TFL force from the subject tests was normalized to maximum pre-fatigue forces, and used to scale the size of kinematic curves in the figures below, thereby providing qualitative intensity and timing of TFL activation. 

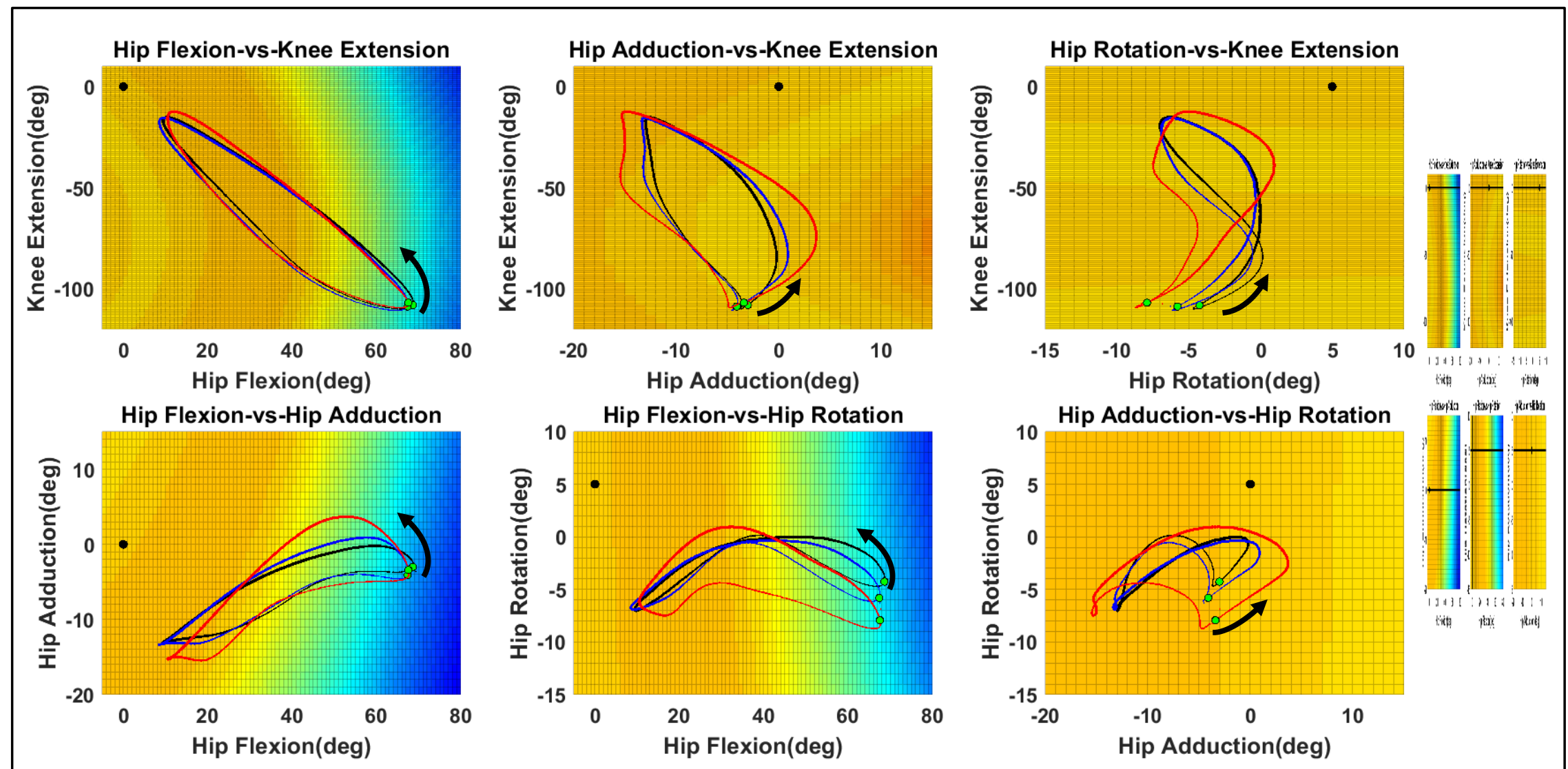

Figure 4-16a: Normalized Fatigue TFL Force Response Surface with Average Kinematic Overlays. Pre-fatigue, intermediate stage, and fatigued/failure kinematics are shown in black, blue, and red, respectively. Green dots and arrow indicate $0^{0}$ crank angles $(12$ o'clock position) and anterior motion of the crank arm. The black dot indicates neutral standing position from Eng 2015. 


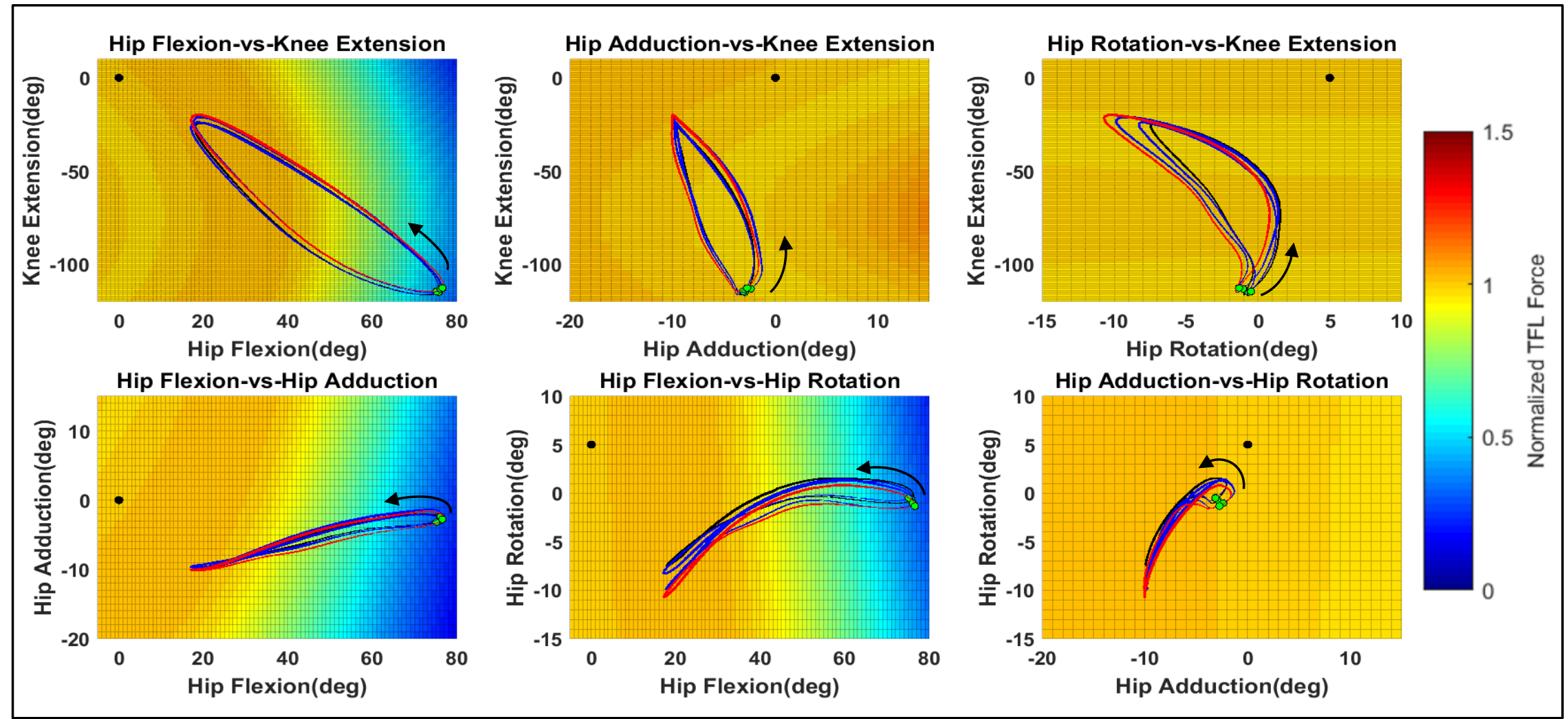

Figure 4-16b: Normalized Non-Fatigue TFL Force Response Surface with Average Kinematic Overlays. Initial, intermediate stage, and final kinematics are shown in black, blue, and red, respectively. Green dots and arrow indicate $0^{0}$ crank angles (12 o'clock position) and anterior motion of the crank arm. The black dot indicates neutral standing position from Eng 2015. 
Through full body CMC, muscle activation can be estimated over the cycle. By applying subjectspecific CMC results to the updated full-body model, average TFL force trends [Figures 4-17a/b] were found for both performance groups.

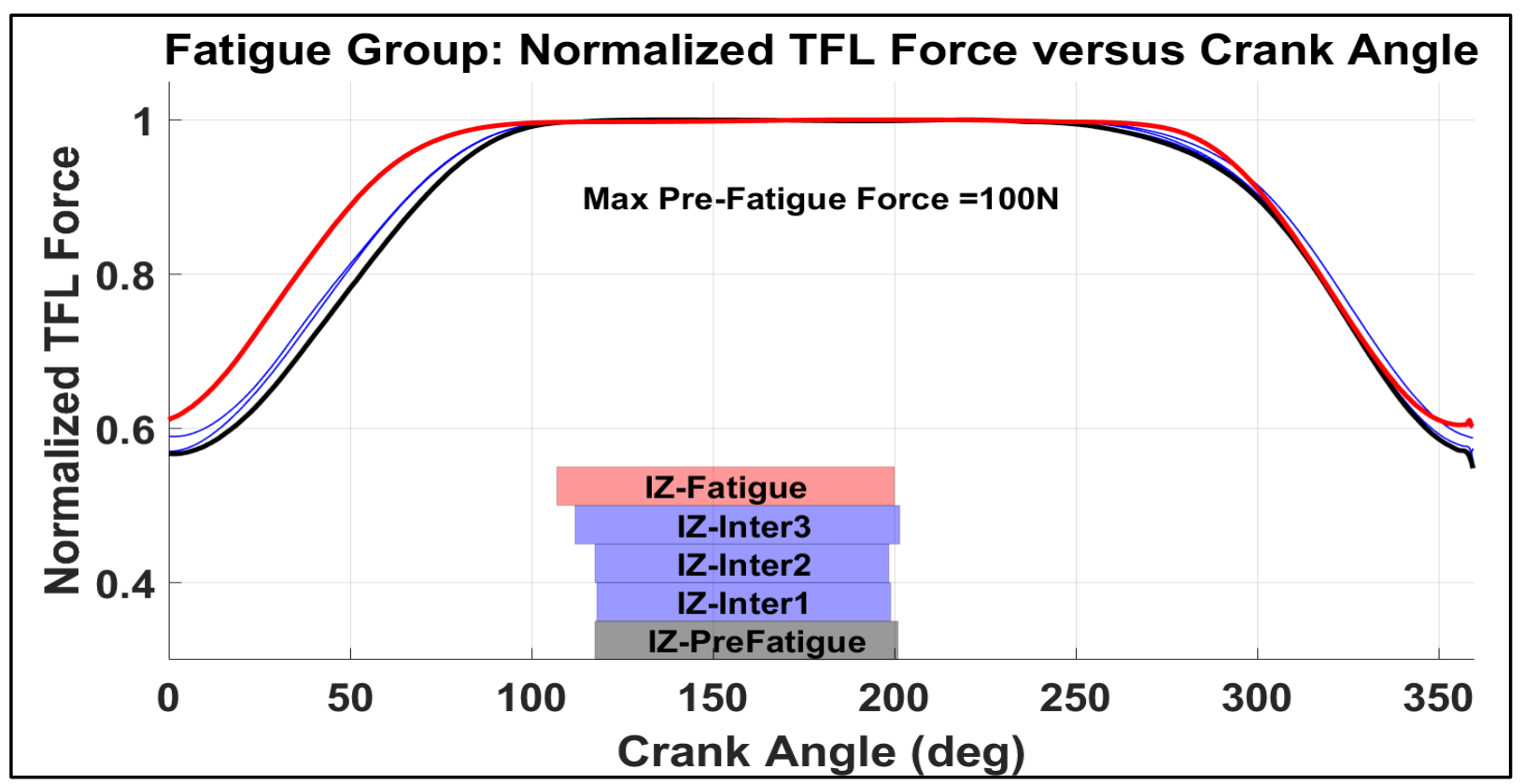

Figure 4-17a: Normalized Fatigue TFL force versus crank angle. Pre-fatigue, intermediate stages, and fatigue/failure are shown in black, blue, and red, respectively. Changing ranges of "Impingement Zone" are also displayed along the $\mathrm{x}$-axis.

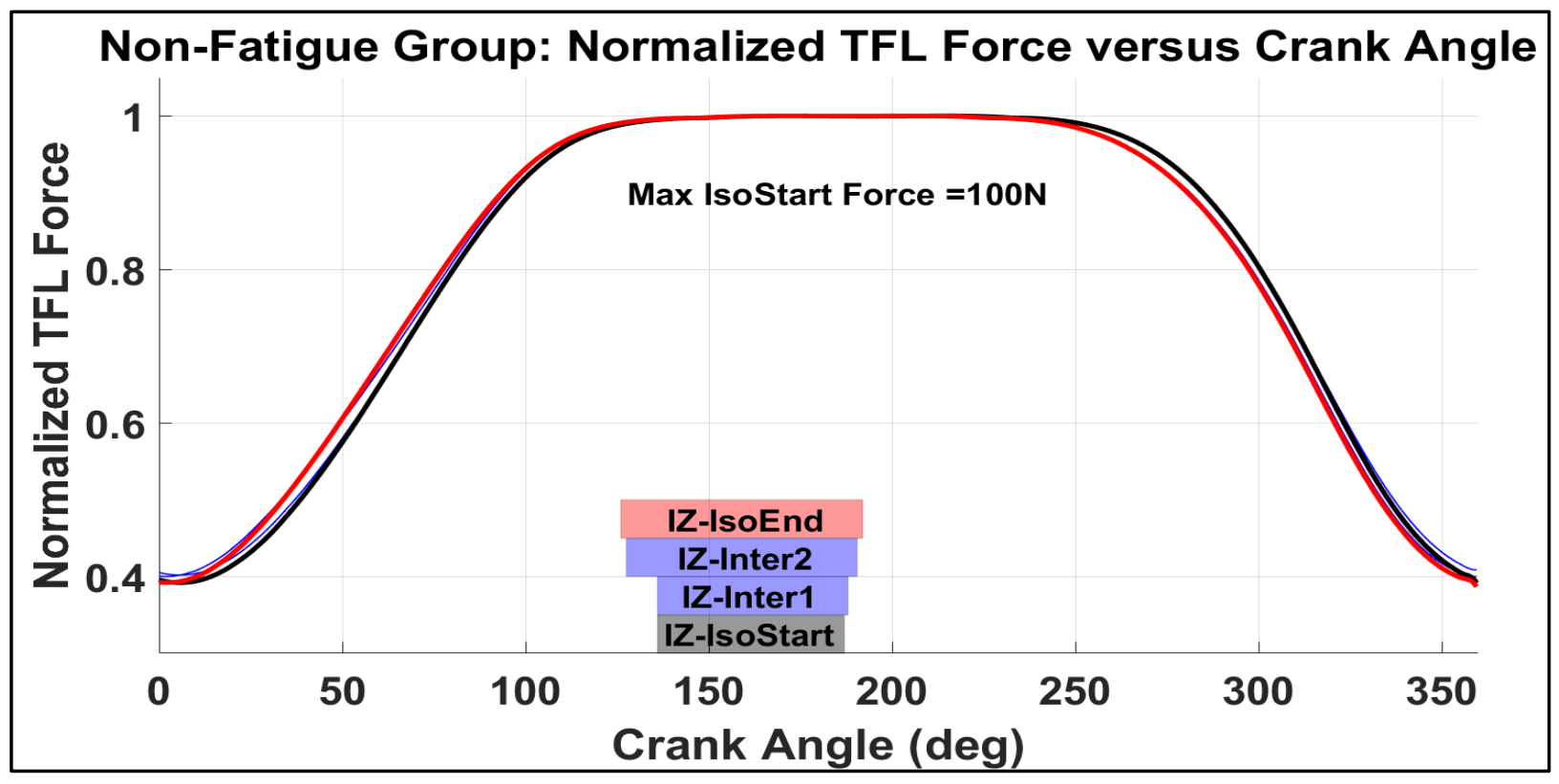

Figure 4-17b: Normalized Non-Fatigue TFL force versus crank angle. Initial, intermediate stages, and final are shown in black, blue, and red, respectively. Changing ranges of "Impingement Zone" are also displayed along the $\mathrm{x}$-axis. 
By observing the predicted nominal on/of cycle of the TFL, and subsequent changes over the ride, more informed interpretations of the LFE compression force estimates can be made. Both groups exhibited max TFL force between mid-downstroke through to mid-upstroke. Initial Fatigue group activation shows greater activation duration than the Non-Fatigue subjects. In addition, the minimum observed force was significantly higher for the Fatigue group (60\% Max TFL Force) versus the Non-Fatigue group (40\% Max TFL Force). At the end of the ride section, a significantly earlier onset of TFL activation is seen among Fatigue subjects, along with an increase of minimum TFL force of $\sim 5 \%$ Max Force. Non-Fatigue subjects did not express any significant change with continued cycling. While fatigue-induced TFL changes occurred in one group, increased IZ duration is in seen in both groups.

Utilizing the force transfer curves from LFE model development results (4.3.1), the proportion of TFL force transferred to LFE compression could be calculated. Since LFE compression curves are dependent on knee flexion (due to rigid ITB attachment superior and inferior to lateral knee), an estimate of LFE compression force was found by combining knee flexion and TFL force for each subject. Compression force estimates are presented in Figures 4-18a/b.

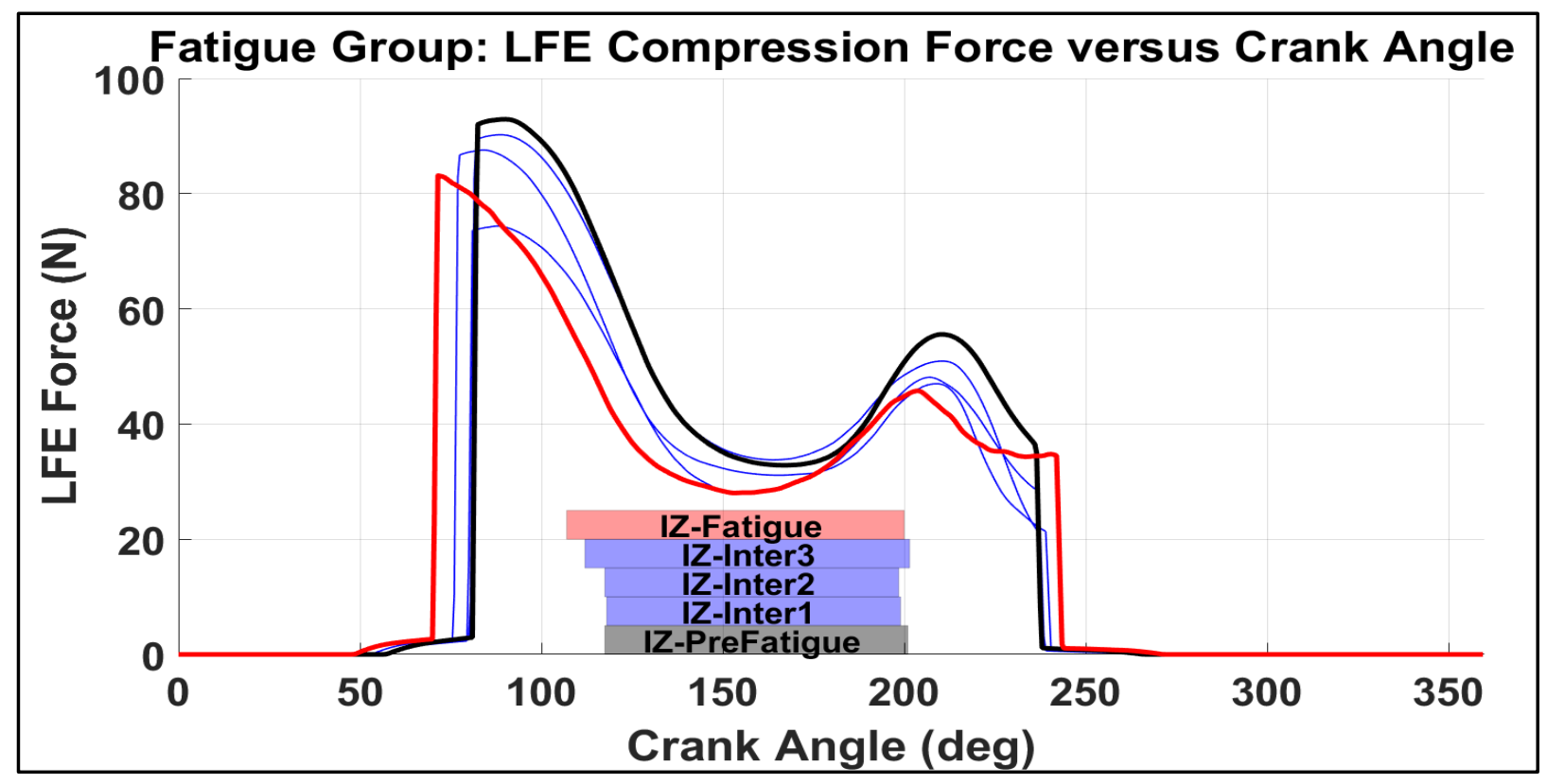

Figure 4-18a: Predicted Fatigue LFE compression force versus crank angle. Pre-fatigue, intermediate stages, and fatigue/failure are shown in black, blue, and red, respectively. Changing ranges of "Impingement Zone" are also displayed. Forces are generated from a TFL model limited to $100 \mathrm{~N}$ max force for direct comparison to kinematic effects in the Non-Fatigue group. 


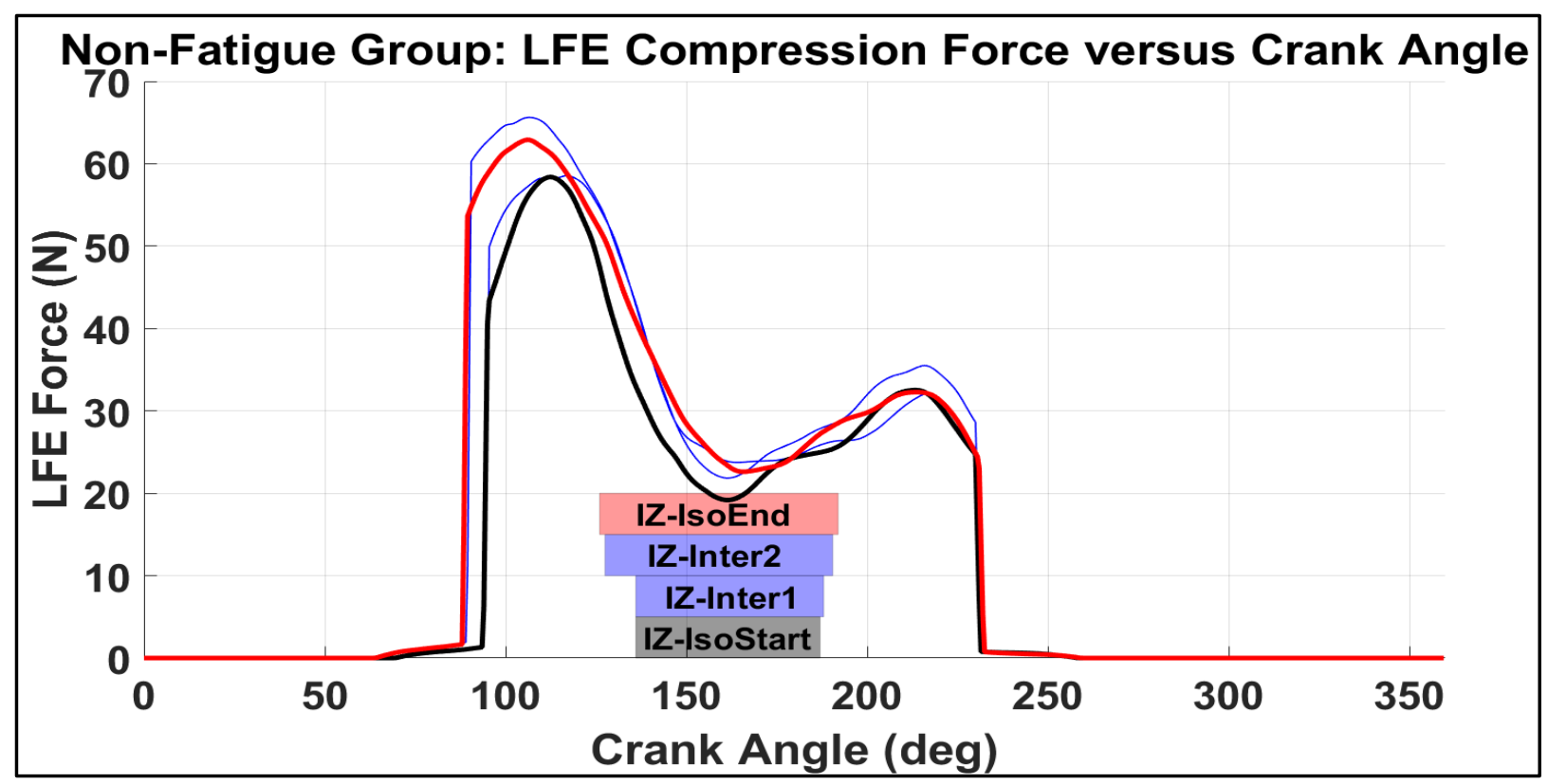

Figure 4-18b: Predicted Non-Fatigue LFE compression force versus crank angle. Pre-fatigue, intermediate stages, and fatigue/failure are shown in black, blue, and red, respectively. Changing ranges of "Impingement Zone" are also displayed. Forces are generated from a TFL model limited to $100 \mathrm{~N}$ max force for direct comparison to kinematic effects in the Fatigue group.

LFE compression estimates for both groups exhibit a bimodal behavior over the crank cycle and ride sections. Compression peaked in mid-to-late downstroke and early upstroke. In addition, literature-predicted IZ occurs between peaks. Initial Fatigue group showed greater magnitude and earlier onset of the first peak LFE compression force than the Non-Fatigue subjects. Secondary upstroke LFE was greater for the Fatigue group, but timing was similar between groups. Varying along with performance reduction, Fatigue subjects exhibited a decrease in LFE compression force, as well as extended LFE contact duration during mid-downstroke and mid-upstroke. Peak LFE forces also shifted earlier in the cycle by $10^{\circ}-15^{\circ}$ in the Fatigue subjects. Non-Fatigue showed an increase in downstroke peak LFE compression, with an earlier average onset. Upstroke peak compression did not exhibit significant changes with continued cycling.

It is observed that OpenSim modeled LFE compression duration is significantly greater than that of literature-predicted IZ. After tracking IZ for each performance group and ride section, onset/offset timing and duration of IZ was analyzed. Results from the literature-defined IZ analysis are presented in Figure 4-19. 


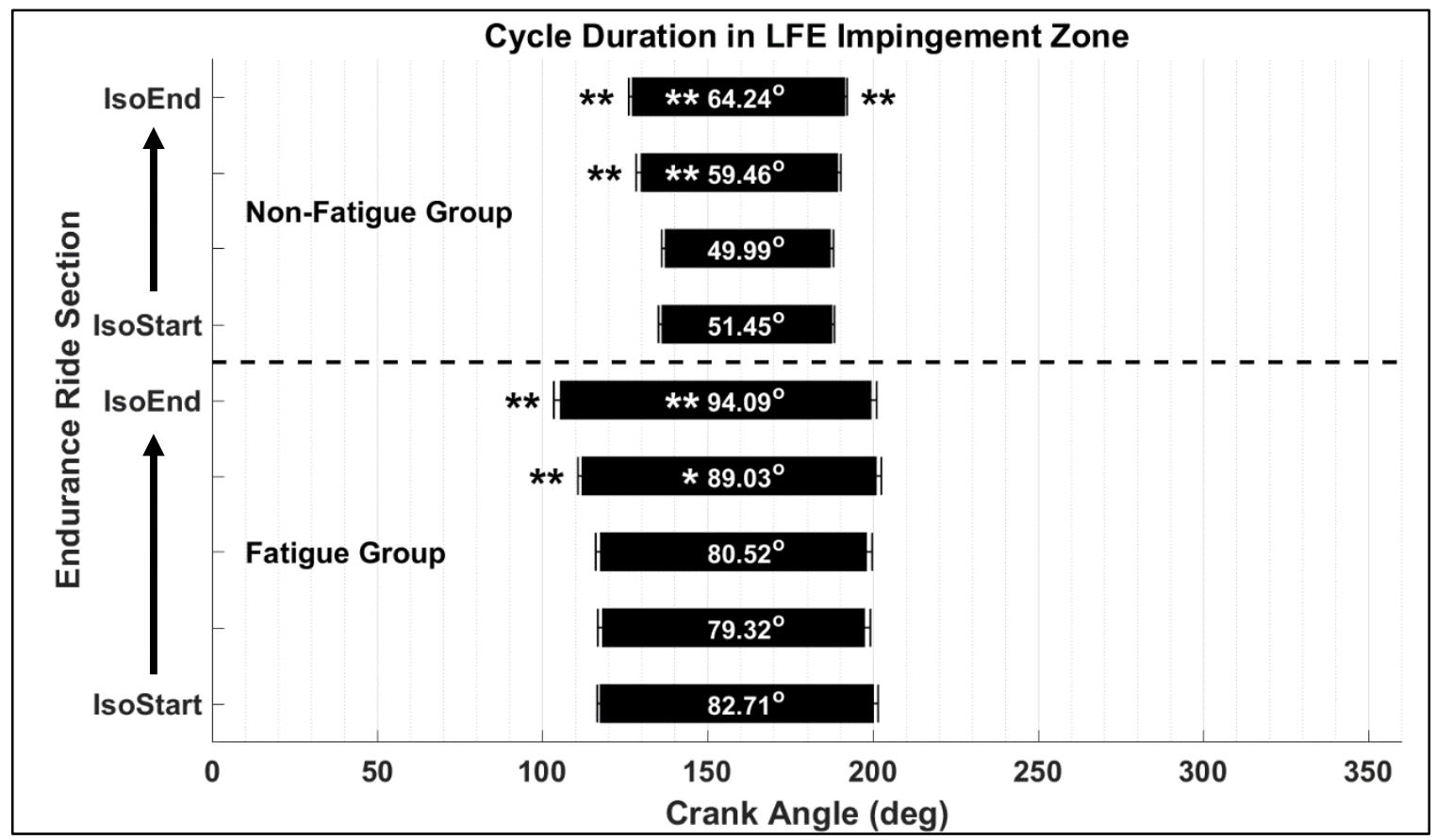

Figure 4-19: Impingement Zone Range-of-Motion Changes for Non-Fatigue and Fatigue Volunteer Groups. Significant changes in the IZ-start, IZ-end, and IZ duration are indicated by asterisks on the respective end or on the average duration bar $(* \mathrm{p}<0.05$, and $* * \mathrm{p}<0.01)$.

Fatigue subjects consistently exhibited greater IZ duration, earlier onset, and later offset in the cycle compared to Non-Fatigue subjects. However, Fatigue groups only showed significantly earlier onset with performance reduction $(\mathrm{p}<0.01)$, while Non-Fatigue subjects displayed later offset of IZ ( $p<0.01)$, as well. Cycle duration in IZ found to be significantly greater in Fatigue subjects, but both groups had significant increases in IZ duration over the ride $(\mathrm{p}<0.01)$.

\subsection{DISCUSSION AND CONCLUSIONS}

\subsubsection{Model Evaluation AND BiOFIDELITY}

Baseline OpenSim MSK models use approximate anatomical landmarks for muscle paths and geometry, but paths are often rigidly bound to the skeletal structure. Static muscle paths limit biofidelity of muscle predictions in dynamic kinematic simulations. Through the presented model development, wrap surfaces and via points were implemented in a base OpenSim MSK model to approximately match tensor fascia latae (TFL) moment arm to cadaveric data along 1-DOF hip and knee ranges of motion. In instances where this study's model TFL moment arm deviated from 
the corridor, effort was made to target the final moment arm curve from other literature models. Once muscle geometry was addressed, an assessment of the Thelen 2003 muscle definition was made through static pose cycling of muscle activation and fiber length. From this evaluation, the max muscle force $(100 \mathrm{~N})$ is not obtained with full activation in the standing position. In addition, the magnitude of active TFL force is directly proportional to the activation level, with a residual level of passive tendon force throughout the test. Fiber length was not seen to significant effect muscle force. Using this force-limited muscle model at full activation, a series of 1-DOF and 2DOF simulations were run to characterize the effect of joint kinematics (muscle lengthening) on TFL force. With the developed muscle path, hip flexion was found to strongly influence total muscle force, resulting in over $70 \%$ force reduction when moving from standing to an 80 -degree flexed position. In 1-DOF models, hip adduction had the second greatest effect, but only contributing to force range of $+3 \%$ at 15 degrees abduction to $-2 \%$ at 15 degrees adduction. Increasing knee flexion exhibited a slight effect on TFL force, with at most $2 \%$ force reduction at approximately 35 degrees and 110 degrees flexion. Internal hip rotation did not significantly affect constant activation force. The 1-DOF observations translated directly to 2-DOF TFL force characterization, where hip flexion primarily drove muscle lengthening. However, through visualization of coupled kinematics, hip adduction and knee flexion are seen impose a larger effect through modulation of TFL force change with hip extension. The interplay between kinematics and the non-sinusoidal nature of muscle lengthening indicates a level of biofidelity has been added to the base OpenSim model. Force response surface mapping facilitates relating joint kinematic and muscle kinetics when evaluating subject-specific data.

Following TFL mapping, definition of the lateral femoral epicondyle (LFE) was needed to estimate LFE compression forces (a risk factor for ITBS). Two methods were explored in this study: explicit and implicit LFE contact modeling. Both methods were built from the TFL moment arm matched model, in which the lateral knee contact surface was defined. Implicit modeling (Model 1) allowed for a numerically smooth motion of the TFL over the lateral knee, but did not allow for modeling of the loading transition from the lateral knee to the LFE. Explicit modeling (Model 2) was guided by MRI-data to improve biofidelity, but resulted in muscle-surface contact issues and significantly increased computational time. With instability occurring, the effect of explicit contact on TFL force across the full range of motion was not known. To reduce the confounding effects of explicit 
modeling on muscle predictions, a hybrid approach utilizing both LFE model types was implemented. Computational time was reduced by using implicit LFE definitions when running subject-specific models, but using a linear interpolation function from the explicit LFE force profile to translate estimated TFL force to LFE compression force. This method allowed for contact-related muscle instability to be removed from the model, while preserving the biofidelity of an explicitly defined region of LFE contact.

\subsubsection{LFE COMPRESSION EXPOSURE}

When evaluating LFE compression with the developed MSK model, two main factors contribute to the magnitude and duration of exposure: kinematics and TFL force. As shown in Chapter 2, both the Fatigue and Non-Fatigue groups exhibited kinematic changes with continued cycling. For the Fatigue group, TFL force response surfaces indicate that fatigue-induced changes in knee extension and downstroke hip adduction patterns put the TFL in a greater force generating position. As for the Non-Fatigue group, osteokinematics remain stable with continued cycling, with only increased hip external rotation leading to slightly increased duration of the TFL in a high force generating position. These kinematic changes are shown to influence the normalized TFL force, where downstroke TFL force is shown to significantly increase with the onset of Fatigue. The Non-Fatigue group does not show a significant change in the TFL kinetics over the ride segments. In addition, the Fatigue group is predicted to have significantly greater TFL force throughout the cycle compared to the Non-Fatigue group. It is of interest to note that the changes in TFL force duration do not appear to correlate with changes in the "Impingement Zone", as defined in literature (Hamill et al. 2008). This indicates that TFL force independently translate to increased LFE compression risk. Coupling the kinematic changes with the normalized TFL force, a prediction of LFE compression force over the cycle can be made.

LFE compression force is predicted to peak twice over the crank arm cycle: as the knee extends during late downstroke, and as the knee flexes during early upstroke. In the Fatigue group, exposure to the LFE compression force was predicted to increase with the onset of fatigue-induced kinematic changes. While threshold values for LFE soft tissue damage are not available, presented results indicate an increased risk of ITBS development due to increased contact duration at high compression force levels. For the Non-Fatigue group, the LFE contact duration was predicted to 
significantly increase, even with relatively stable osteokinematics over the ride segment. With the increased power output and increased RPM, the peak LFE compression force during the downstroke was estimated to increase. Both performance groups exhibited a significant increase in "Impingement Zone" duration, primarily by earlier onset of knee extension during the downstroke $(\mathrm{p}<0.01)$. Upstroke knee flexion timing was maintained over ride segments for both subject groups ( $p>0.05$ ), except for the final section of the Non-Fatigue ride segment, where the knee began to flex significantly later in the cycle $(\mathrm{p}<0.01)$. Lateral knee compression is largely unexplored in this investigation, which may allow another pathway for injury development associated with ITBS. Predictions from this study hint that the current definitions of the kinematicdependent "impingement zone" may underestimate exposure risk to overuse injury factors.

\subsubsection{ITBS \& OVERUSE INJURY RISK}

The results presented show significant kinematic and LFE loading changes with fatigue and performance increases in stationary biking. With regards to overuse injury, exposure time to ITBS risk factors was predicted to increase with prolonged fatigue. In addition, predicted LFE compression duration was significantly longer than that predicted by literature definition of the LFE "impingement zone" ( $<30^{\circ}$ knee flexion). Reevaluation and improved modeling of ITBS injury risk kinematics is necessary to prevent underestimation of exposure risk. While longitudinal data directly relating LFE compression force and ITBS development is not available, the association between supra-LFE soft tissue inflammation and ITBS is supported through recent radiography literature (Flato et al. 2017; Friedman et al. 2017; Huang et al. 2013).

Frameworks, like that developed in this study, can be used to develop probabilistic overuse injury risk functions for ITBS, as well as patellofemoral pain syndrome (PFPS), and nonspecific chronic lower back pain (NS-CLBP). Fatigue trends in the observed volunteers demonstrate correlations to the possible risk factors presented in the literature. Fatigue-induced increases in hip peak adduction, abduction and internal rotation are also known to be linked with increased occurrence of ITBS and PFPS (Miller et al. 2007; Louw and Deary 2014; Boling et al. 2009; Bailey, Maillardet, and Messenger 2003). Increased flexion of the lower back over time is also associated with greater risk of developing NS-LBP during cycling. These technique changes have the potential, if not monitored and corrected, to cause overuse injuries and subsequent acute injury. 


\section{Chapter 5}

\section{Conclusions}

\subsection{ANALYSIS FRAMEWORK}

From high-dimensional Bayesian models to simplified physical evaluation, many methods are present in literature to address and predict human performance and endurance (Harman et al. 2008; Lisman et al. 2013; Ji, Lan, and Looney 2006). Each modeling approach has strengths and limitation in the desired application, but either require constant-monitoring of several environmental and internal variables, or are not directly generalizable to other activities. The analytical framework and performance insights [Figure 5-1] gained by this research could prove effective in applications such as athletics, national defense, and clinical care. In each application, human performance research offers several areas of innovation: performance prediction, real-time monitoring, injury prevention, and human-machine augmentation. Through the conducted investigation, a preliminary framework has been developed to generate subject-specific data relevant to each area of innovation. Extension of the proposed framework to monitoring devices and algorithm development will allow generation of real-time individualized performance metrics.

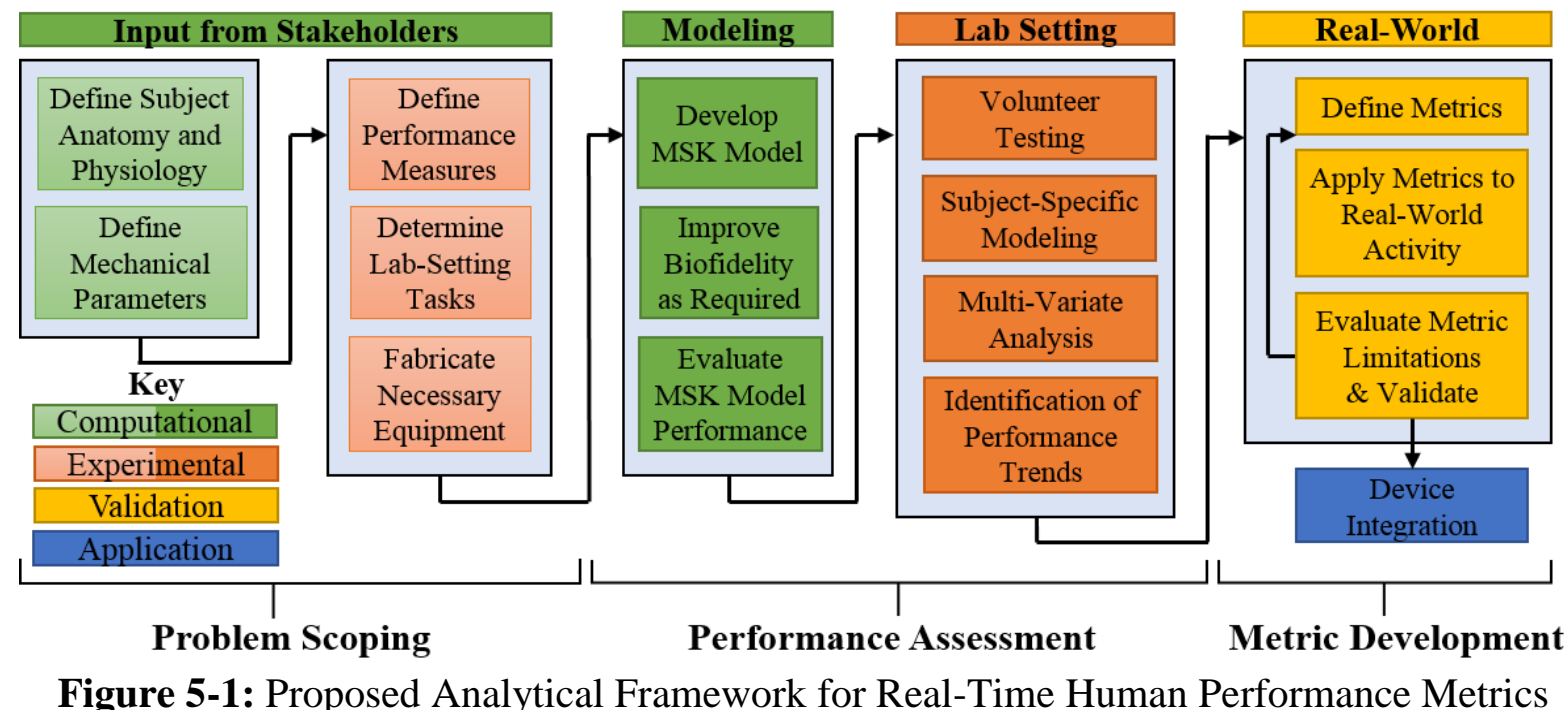




\subsection{FATIGUE, COMPENSATION, AND INJURY}

Overall, system-level performance reduction appears to be accompanied by fine-motor control loss and several subsequent kinematic changes. These muscular and kinematic changes ultimately transfer the system's force generation supply from musculoskeletal power sources to reliance on inertial power sources. While the presented analysis does not include EMG or kinetic performance metrics for the upper body, observed kinematic changes of the torso indicates increased engagement of the upper body and lower back in power generation during fatigued cycling. Fatigue-induced kinematic ROM increases may correlate with increased overuse injury risk of the lumbar spine and knee in cycling. The loss of fine motor control from the tibialis anterior and medial gastrocnemius appear to induce muscular compensation in the force-generating muscles. Rectus femoris (knee extension) and biceps femoris (knee flexion) shift their activation profile to envelope the pre-fatigue active regions of tibialis anterior (ankle dorsiflexion) and medial gastrocnemius (ankle plantarflexion), respectively. Power output profile was preserved, although power magnitude was reduced with muscular fatigue. Observation that power delivery profiles remain relatively constant with fatigue indicates either cognitive or mechanical constraints on system-level kinetic behavior. Additional research can investigate power profile morphology as a performance constraint that kinematics and muscle activations target despite fatigue onset.

Non-Fatigue group performance exhibited kinematic differences when compared to the fatigued individuals (i.e. increased core stability, lower lumbar flexion). Fine-motor control muscles did not reduce activation magnitude in this group, but showed significant shifts in timing with increasing performance level. ITBS injury risk, while correlated with hip and knee kinematic changes in both groups, was also influenced by increased power output in the Non-Fatigue group. MRI-guided OpenSim models developed indicate need to reevaluate current definition of LFE "Impingement Zone", which may underestimate ITBS injury development risk. Maintenance of distal motor control, specifically the clutch-like mechanism of the medial gastrocnemius, correlated strongly with overall performance. Characterization of full body performance trends across body sub-systems is necessary to provide guidance on high intensity sports. The role of perceived effort and discomfort in guiding performance is a knowledge gap of future interest. 


\subsection{EMG-BASED PERFORMANCE MODELS}

Linear regression techniques successfully led to the development of a RPM model form comprised of foot plantar flexion and dorsiflexion muscle parameters. Fine-motor control muscle parameters exhibited strong performance prediction strength compared to power generating muscles. Though potentially limited by EMG signal quality from power-generating muscles, the observation indicates initiation of system-level failure occurring with weakening of distal body segment control, instead of fatigue of power-generating muscles. Generalization of this framework to maintaining long-term performance in other applications would shift focus from power generation strengthening to improved stability control of force application surfaces. While the developed subject-specific models are highly predictive, uniqueness of model fit to each volunteer indicates the need for performance database development and further research on adjusting model form to address the general population variation.

\subsection{CLINICAL RELEVANCE}

\section{Cycling Injury Development and Motor Control}

Current overuse injury development paradigms attribute injury to two error mechanisms: systematic errors and emergent errors. The presented work provides direct commentary on the presence of emergent error pathways in high intensity cycling. All subjects were experienced cyclists with competition training and started tests with proper cycling technique. Once classified based on their ability to sustain performance in this study, the emergence of improper technique is observed with physical fatigue. Fatigue processes, whether leading to performance failure or compensation, induced kinematic changes that are clinically correlated with overuse injury development. Fatigue-induced hip adduction within the Fatigue Group indicates that increased hip adduction or hip abductor weakness, as reported among ITBS cyclists, may be an emergent error (Aderem and Louw 2015; Brown et al. 2016; Noehren, Davis, and Hamill 2007). Therefore, training intensity must be adjusted to avoid relative hip abductor weakness and minimize risk of ITBS development. While compensation mechanisms in the Non-Fatigue Group allow performance maintenance, behavioral changes may still increase exposure to overuse injury due 
to preferential loading of untrained muscles and high-risk osteokinematics. To effectively translate in observed kinematic and kinetic changes to injury development, the definition of overuse injury factors must be refined for each joint pathology. Tissue-level investigations are needed to elucidate the relative weighting of joint stressor intensity and stressor duration in driving long-term injury development.

In addition to long-term injury implications of muscle weakness and joint kinematics, sub-system motor control can be leveraged to understand long-duration task performance. Tibialis anterior and medial gastrocnemius loss in the Fatigue Group and strong correlation of medial gastrocnemius in overall performance indicates that these distal fine-motor control muscles must be emphasized in cycling training. Generalization of this finding hints that end-point control muscles guide longduration system performance. Investigation of how this control-performance relationship holds for other muscle groups and activities can equip practitioners with insight for minimizing emergent error pathways through improved training and human augmentation.

While results from the presented study are translatable to stationary biking and cycling, the experimental framework, computational approach, and key findings can be generalized to other applications. This is made possible by the identification of key muscle groups and activation parameters correlated with overall system-level performance. The opportunity to target analogous muscle groups in other activities (e.g. upper extremity forearm muscles for throwing tasks), will allow diverse application of study findings.

\section{Athletics Safety}

In the increasingly competitive world of high performance sports, the slightest advantage can dramatically influence tournament outcomes. High intensity training and stiff competition pushes athletes to the edge of human performance. Methods in this study provide a framework for the development of generalized performance models for personalized predictions across activities. Coupling these models with device design, training routines and wearables can target fewer muscle groups while still developing a more robust athlete. Improving sports safety not only improves long-term health of competitors, but can be translated to keeping the active population of health- 
conscious citizens safer (Ha et al. 2015). Preventative safety measures in sports will have broad impacts in reducing the overuse injury burden on health care infrastructure.

\section{Military Safety}

MSK health concerns in sports are translatable to military training and in-field injury, as well. Overuse injuries to the knee, neck and spine are among the top causes of pain among recruits in training (Springer and Ross 2013). Similar MSK damage on field not only reduces soldier performance, but also puts their lives at heightened risk. Insights from this study can be used by military doctors and personnel to optimize training regimens to a soldier's body type and muscle distribution. For example, accounting for relative leg length and muscular fatigue trends, recruits could be directed to muscle-specific conditioning to maximize performance stamina for given training time. For combat, computational methods presented can be used to develop real-time monitoring of solider physiology and fatigue. A potential method for this could entail coupling MSK models (e.g. OpenSim) with tracking of key osteokinematics (via optical or inertial means) (Machado, Flores, and Fregly 2011; Sherman, Seth, and Delp 2010; Menard, Domalain, and Lacouture 2014). To overcome on-field data processing limitations, additional research in formulating performance metrics with minimal dependent variables could be more effective than full-body MSK modeling. Data obtained from active soldiers allows for identification of

performance weak points or risky kinematic patterns, opening the opportunity to autonomously correct kinematics and provide gait assistance. Bigger picture application of the analysis framework can provide military strategists decision making power to best fit personnel MSK abilities to mission terrain and task requirements, while minimizing injury risk.

\section{Rehabilitation}

Despite interventions and preventative measures employed in sports and defense, athletes and military personnel will still develop injuries. With performance tracking before injury, MSK rehabilitation progress metrics can be developed relative to each patient's baseline gait parameters, like the modeling approach in this study. Data collected during injury development can aid rapid triaging and diagnosis of injury. Improved diagnostic power allows tailoring of rehabilitation 
techniques to best address the patient's damage without instigating further complications, thereby reducing decommission time, and accelerating return-to-play timelines.

\section{Performance Optimization}

While short term application can improve preventative and diagnostic measures for MSK injury, more thorough understanding of body coordination and system-level fatigue can guide development of performance optimization strategies. Opportunities exist in the definition of realtime performance metrics from the identified distal motor control muscle groups, including algorithms for actuated exoskeletons or body-segment-specific injury risk alerts. Human performance research can guide athletes, soldiers, and patients to execute the optimal musculoskeletal patterns to quickly, efficiently, and safely navigate their environments. 


\title{
Chapter 6 Limitations \& Extensions
}

The presented study of fatigue in stationary biking provides a subject-matched and synchronized collection of kinematic, kinetic, and physiological data over the endurance ride of four "Fatigue" volunteers and three "Non-Fatigue" volunteers. Although 7-of-13 volunteers were used for this analysis, the use of cyclic bilateral measures allowed for statistically significant variations to be detected between the pre-fatigue and fatigue states. Incorporation of OpenSim for osteokinematics and joint force measurements improved the depth of analysis with minimal propagation of error through the OpenSim pipeline. Limitations in the study, however, can be identified at various stages of the experimental setup, data collection, and data analysis.

\subsection{VOLUNTEER SELECTION}

\begin{abstract}
Abdominal Adipose Tissue
While volunteer selection screened out individuals with limited cycling experience, current health concerns, and incompatible anatomy with bike design, adipose tissue distribution requirements were not defined. Inclusion of volunteers with abdominal adipose tissue significantly reduced the EMG signal-to-noise ratio on abdominal muscles, effectively disqualifying collected abdominal EMG data from analysis. Current data from OpenSim shows significant changes in torso kinematics with fatigue, which encourages further investigation on abdominal muscle coordination, performance, and NS-CLBP development.
\end{abstract}

While selection criteria limited volunteers to experienced cyclists with recent or ongoing training regiment, significant variation was seen among the sample of athletes (i.e. practice intensity, prior injuries, body geometry). Of the 13 subjects tested, four exhibited performance fatigue changes similar to that characteristically seen in literature (Brown et al. 2016; Srinivasan and 
Balasubramanian 2007; Rodrigo Rico Bini, Diefenthaeler, and Mota 2010). Approximately half of the individuals outperformed set resistance levels, exhibiting relatively small cycling cadence reduction, even with incremental resistance increases, indicating a potential fatigue compensation mechanism. If volunteer specific characteristics are found to correlate with performance outcome (fatigue vs. compensation), the finding can be used to implement additional pre-selection criteria to tailor volunteer pools to future research questions of interest. In addition to performance variation, body geometry and adipose tissue distribution introduced a limitation into EMG data collection by reducing EMG signal strength and increasing motion artifacts. Limiting the impact of body geometry on EMG quality can be done through additional anthropomorphic selection criteria, including: body fat content and height-to-weight ratio. Implementation of the above volunteer selection adjustments in future studies will allow for more robust selection protocols and improvement of data quality across the subject cohorts for post-processing.

\subsection{VOLUNTEER INSTRUMENTATION}

\section{Marker Clusters, and Effects of Sweat/Motion on EMG \& Vicon}

To define the time-history of rigid body motion, joint coordinates for a mapped system, or global coordinates of three rigidly mounted body points are required. For full-body modeling, OpenSim recommends a 46-marker layout, which tracks at least three points per body segment, and explicitly defines knee and ankle joint centers with two mediolateral points. Although this configuration has redundancies, consistent placement and adequate fixation of the markers is necessary to reduce tracking error. To accommodate the various geometries of participants, anatomical landmarks were used to place and fix each marker individually. While individual placement was more precise, use of a flexible marker cluster for lower extremity and torso tracking would allow more secure attachment and more consistent relative placement between markers. Cluster use would be particularly advantageous in reducing marker wobble and detachment due to soft-tissue motion and sweat. Sweat and motion artifacts also reduced EMG signal quality, thereby limiting the significance of statistical analyses.

Current methods for efficiently attaching Vicon markers to the volunteers consisted of double sided adhesive strips and anatomic landmarks for proper placement. To prep the volunteers, a 
series of steps to remove hair, mark placement, and sterilize areas of instrumentation were done to reduce marker wobble and loss. Even with precautions, markers were still lost during the tests, consequently increasing error in calculations or even invalidating subject data from analysis. Improvements to the Vicon marker securement could be made through construction of skin-tight marker suits or use of marker clusters for tracking of body segments (i.e. distal thigh, torso, shank). To reduce motion artifacts in EMG, improved fixation, in addition to double-sided take and athletic wrap/tape, should be used. In addition, measurement of skin impedance changes caused by sweat can increase reliability of EMG data during high intensity performance.

\subsection{BIKE INSTRUMENTATION}

\section{No Loadcells on Bike Handles for Upper Extremity Analysis}

Instrumentation of the bike included Vicon markers, a crankarm velocity gate, and pedal/seat loadcells. With these modifications, pedal/seat position, orientation, and reaction forces were measured throughout the endurance ride, allowing for lower extremity performance analyses. However, to extend analysis to upper extremity and abdominal kinetics, reaction forces at the bike handles must also be collected. Without this data, estimation of lower back and abdominal muscle forces through OpenSim would be significantly overpredicted, limiting analysis of the upper body to kinematic parameters in this study. Implementation of handle loadcells in the current volunteer study was avoided due to significant loadcell damage concerns - primarily, risk of accidental excessive loading of the handles during volunteer mounting and dismounting from the bike could easily exceed max torque thresholds of loadcells available at the laboratory. Future studies should first address handle loadcell design concerns to allow research extensions to upper body metrics.

Current configuration of the stationary bike includes instrumentation of the pedals, seat, and crank arm for direct measurement of lower body reaction forces and calculation of RPM and kinematics. With this setup, data analysis was limited to lower body kinetics and kinematics. Inclusion of handle bar reaction forces and motion would provide additional boundary condition information such that upper body analyses could be conducted. Given the large moment arm of bicycle hands, integration of handlebar load cells would pose significant design hurdles while maintaining sufficiently safety factors for load cell damage in bending. Potential solutions for handlebar 
instrumentation include interval-placement of load cells along the handle, but would subsequently limit placement of hands to the load cell loading surface. Refinement of Vicon marker placement for the handles would also be required as to accurately track the additional upper body loading surfaces on the handlebars.

\subsection{TESTING PROTOCOL}

\section{Resistance Setting and Subsequent Adjustments were User-Specified}

To rigorously test cycling performance, knowledge of the bike's resistance force or friction coefficient is key. Commercially available magnetic-resistance stationary bikes allow for repeatable and incremented resistance settings, but budgetary limitations in this study prevented use of such a device. Instead, a friction-pad stationary bike was purchased and instrumented for used in the study. Project timeline limitations prevented design of adequate instrumentation for measuring real-time compressive force of the friction-pad and quantification of the friction-pad's dynamic friction coefficient. Considering these instrumentation limitations, the test was designed to mimic a real-life instructed group stationary biking session with self-selected resistance on a 1to-10 scale of perceived difficulty. Like real-life stationary biking group session guidance, instruction was given to adjust the resistance to maintain a pre-defined level of difficulty during the endurance ride. While the chosen methods replicate training experience, the variability of resistances and adjustments between riders significantly reduced the ability to generalize the collected data. Improved instrumentation of the bike is needed to precisely set and monitor resistance force during the test. Though the technique was not possible with available testing time and research facilities in this study, future studies should implement isometric contraction force measurements of the quadriceps and hamstrings to define a volunteer-specific the resistance profile applied to each volunteer. In conjunction with resistance force measurement, the proposed methods will allow investigators to account for strength differences across participants in performance analyses and model development. 


\subsection{FOLLOW-UP SURVEY \& LONGITUDINAL TRACKING}

\section{No Tracking of Volunteer Pain Incidence Following Testing}

While attention was given to minimizing cardiovascular and muscular injury risk to the volunteers during this study, participants were pushed to significant levels of performance failure during the endurance ride. Termination of the trial before the allotted 67.5-minute time limit was voluntary and participants were reminded of their ability to end the trial at any time, and rise concerns. Although no negative reports of musculoskeletal pain were received after testing, tracking of individuals' pain symptoms would strengthen commentary on observed performance changes and injury risk. Addition of a follow-up survey one-to-two weeks after the participants' test session should be implemented in future volunteer test protocols as an effective method to collect relevant symptomatic data.

Introduction of longitudinal tracking in this study can take two forms: follow-up surveys after a single testing session, or repeated testing sessions on an annual basis. Information from these methods would substantiate any indications of injury risk predicted by computational modeling. Future extensions of related research can implement either method to allow commentary on longterm injury and will add to the limited longitudinal literature on overuse injury development.

\subsection{OPENSIM ANATOMIC CONSIDERATIONS}

\section{ITB-Kaplan Fiber Laxity and Explicit LFE Definition}

Current rigid body modeling tools for human musculoskeletal dynamics are tailored for improved computational speed to allow direct application to real-time analyses. Improved computational cost, however, often comes with a reduction of model biofidelity. To estimate dynamic TFL and LFE forces, muscle path laxity and contact geometry must be introduced into base OpenSim models. While a rigorous approach was used to match the TFL muscle path and LFE contact surface with current literature, the model still lacks some biofidelity considerations. Recent cadaveric dissection literature on distal ITB attachments quantitatively defines the origins, attachments, stiffness, and dissected distances of the proximal and distal Kaplan Fibers. These fibers, addition to the linea aspera, provide distal attachment of the ITB. While knowledge gaps 
exist for scaling mechanical properties of these connective tissues for user-specific OpenSim models, explicit modeling of these tissues will allow more biofidelic modeling of ITB kinematics and kinetics compared to the current rigid distal femoral ITB attachment definition in this study. In addition, explicit LFE contact geometry definition was guided by current MRI literature in symptomatic ITBS patients, potentially reducing applicability of the model's LFE force predictions to only ITBS injury development. For more accurate LFE compression force estimation, future investigations should develop an iterative OpenSim-FEA analysis based on userspecific CT reconstruction of lateral knee geometry and mechanical property scaling of the ITB and joint connective tissues.

Standard OpenSim modeling available are limited in biofidelity of muscle path and laxity for prediction of muscle-bone forces. Steps were taken to develop a subject-scalable model with improved TFL laxity characteristics and MRIguided definition of LFE contact surfaces. Recent anatomical literature on distal femoral ITB attachment has quantified geometry and mechanical properties of the Kaplan Fibers [Figure 6-1] (Flato et al. 2017). Extension of the current model for improved biofidelity should attempt to integrate Kaplan Fibers (e.g. via linear

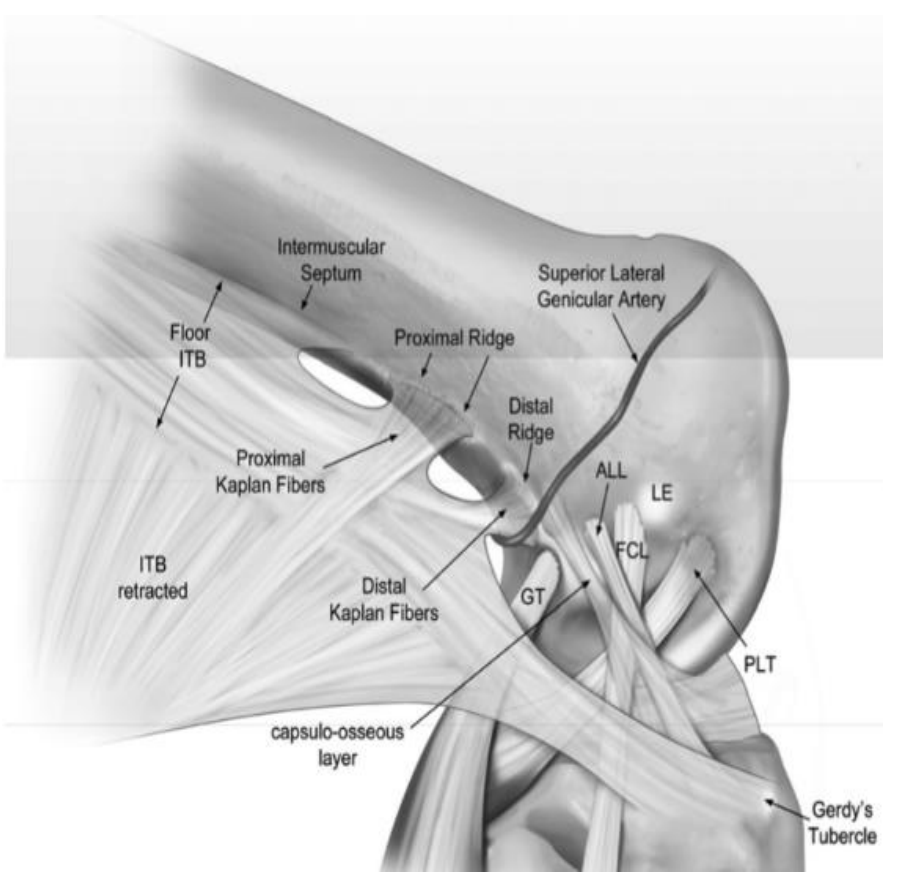

Figure 6-1: Distal Femoral Attachments of the ITB spring elements) to allow for additional degree of TFL motion during dynamic modeling. Given proximity of the Kaplan Fibers to LFE contact surfaces, even laxity of the ITB distal femoral attachments will have significant effects on LFE compression force modeling and ITBS risk factor exposure predictions.

For full body modeling, addition of the upper extremities and spine will allow for a more accurate osteokinematic and center of mass tracking. Given relatively large moment arm of the head mass 
in a hunched over position, like that seen at fatigue/failure, neck flexibility in the model will greatly influence abdominal muscle activity and joint forces when extending research methods to upper body and lower back modeling.

\subsection{OPENSIM PIPELINE}

\section{RRA Implementation and TFL Force Prediction}

Prior to CMC, OpenSim's RRA (Residual Reduction Algorithm) is a modified forward dynamics tool that is typically used to de-noise kinematic/kinetic data and assist convergence of CMC muscle optimization solutions. In this study, RRA was not able to be successfully implemented prior to $\mathrm{CMC}$, potentially due to the boundary condition constraints or excess noise levels in the empirical input data. Since RRA is intended for use in walking/running gait modeling (where center of mass translates globally), the stationary aspect of the current model may be leading to the observed optimization issues. Without RRA, CMC results are likely to have increased error, thereby reducing reliability of predicted TFL forces from this model. While significant changes were still seen in TFL/LFE forces, additional work must be done to implement RRA into the presented OpenSim analysis pipeline.

Computational methods implemented through OpenSim took advantage of the built-in "Scaling", "Inverse Kinematics" (IK), "Inverse Dynamics" (ID), "Computed Muscle Control” (CMC), and "Analyze" tool sets. With this pipeline, volunteer-specific empirical data was used to generate secondary parameters not directly measured in the cycling session. Computational methods are commonly susceptible to error propagation from each discrete time step and between modeling stages. Error propagation was observed in OpenSim's low Inverse Dynamics error (max error of approximately $0.08 \%$ ), but significant levels of CMC-EMG discrepancy output from the downstream analysis pipeline. OpenSim provides two mechanisms for limiting computational error in CMC: "Reduced Residual Analysis" (RRA) and empirical EMG matching protocols. RRA is a built-in CMC pre-processor that is used to reduce discretization error from IK/ID outputs to improve the stability of the CMC optimization. EMG data matching within CMC provides the optimization loop an empirically observed initial guess and aligning CMC and EMG results. RRA 
and EMG data matching were not implemented in the presented computational analysis, but will be used to reduce computational error in future modeling efforts.

\subsection{PERFORMANCE MODEL GENERALIZATION}

Conclusions from performance modeling in this study show importance of tibialis anterior and medial gastrocnemius in predicting overall cycling performance. The relative strength of fine motor control muscles compared to power generating muscles provides a crucial step towards development of non-activity-specific low-computational cost performance models. While models are predictive, subject-specificity of model coefficients needs to be addressed in future investigations. Given wide variation of physiological and anatomic in the human population, implementation of principal component analysis and supervised machine learning methods on large human performance fatigue datasets will allow for the subject-specificity of current models to be addressed. Once a performance fatigue model is developed, further generalization can be achieved through:

\section{1) Consideration of compensation mechanisms,}

2) Consideration of psychological motivation and intent effects.

Future research should aim to integrate the above approaches to generalize and validate the resultant model across a multitude of activities.

\subsection{FINITE ELEMENT KNEE MODEL}

Computational methods used in the presented study are founded upon rigid-body assumptions for bone and joint contact surfaces. Obtaining deeper clinical power of the data can be achieved via finite element (FE) approaches for analyzing joint dynamics. Real-time applications of FE are limited by high computational time. However, through development of iterative multibody-FE coupled models, the problem complexity can be significantly reduced. Primary hurdles exist in addressing non-invasive kinematic-tracking bone, total joint-capsule material characterization, and accurate modeling of muscle activation force distributions. As these knowledge gaps are bridged, coupled multibody-FE methods can move the field closer to real-time overuse injury metrics and human-machine device integration in sports, military, and health care applications. 


\section{References}

Abbiss, Chris R, Jeremiah J Peiffer, and Paul B Laursen. 2009. "Optimal Cadence Selection during Cycling.” International SportMed Journal 10 (July 2015):1-15.

Abt, John P., James M. Smoliga, Matthew J. Brick, John T. Jolly, Scott M. Lephart, and Freddie H. Fu. 2007. "Relationship Between Cycling Mechanics and Core Stability." Journal of Strength and Conditioning Research 21 (4):1300-1304.

Aderem, Jodi, and Quinette A. Louw. 2015. "Biomechanical Risk Factors Associated with Iliotibial Band Syndrome in Runners: A Systematic Review." BMC Musculoskeletal Disorders 16 (1):356. https://doi.org/10.1186/s12891-015-0808-7.

Bailey, Martin P., Frederick J. Maillardet, and Neil Messenger. 2003. "Kinematics of Cycling in Relation to Anterior Knee Pain and Patellar Tendinitis." Journal of Sports Sciences 21 (8):649-57. https://doi.org/10.1080/0264041031000102015.

Baker, Robert L., Richard B. Souza, and Michael Fredericson. 2011. "Iliotibial Band Syndrome: Soft Tissue and Biomechanical Factors in Evaluation and Treatment." $P M \& R 3$ (6). Americal Academy of Physical Medicine and Rehabilitation:550-61. https://doi.org/10.1016/j.pmrj.2011.01.002.

Bernardo, Nieves de, Carlos Barrios, Pablo Vera, César Laíz, and Michal Hadala. 2012. "Incidence and Risk for Traumatic and Overuse Injuries in Top-Level Road Cyclists." Journal of Sports Sciences 30 (10):1047-53. https://doi.org/10.1080/02640414.2012.687112.

Bini, Rodrigo, Patria A. Hume, and James L. Croft. 2011. "Effects of Bicycle Saddle Height on Knee Injury Risk and Cycling Performance.” Sports Medicine 41 (6):463-76. https://doi.org/10.2165/11588740-000000000-00000.

Bini, Rodrigo R., Frederico Dagnese, Emmanuel Rocha, Mateus C. Silveira, Felipe P. Carpes, and Carlos B. Mota. 2016. "Three-Dimensional Kinematics of Competitive and Recreational Cyclists across Different Workloads during Cycling." European Journal of Sport Science, no. JANUARY. https://doi.org/10.1080/17461391.2015.1135984.

Bini, Rodrigo Rico, Fernando Diefenthaeler, and Carlos Bolli Mota. 2010. "Fatigue Effects on the Coordinative Pattern during Cycling: Kinetics and Kinematics Evaluation.” Journal of Electromyography and Kinesiology 20 (1). Elsevier Ltd:102-7. https://doi.org/10.1016/j.jelekin.2008.10.003.

Bini, Rodrigo Rico, and Patria A. Hume. 2012. "Relationship between Pedal Force Asymmetry and Performance in Cycling Time Trial." Journal of Sports Medicine and Physical Fitness 147 (2):135-40. https://doi.org/10.13140/RG.2.1.5129.4169.

Bogert, Antonie J. van den, Thomas Geijtenbeek, Oshri Even-Zohar, Frans Steenbrink, and Elizabeth C. Hardin. 2013. "A Real-Time System for Biomechanical Analysis of Human Movement and Muscle Function." Medical \& Biological Engineering \& Computing 51 (10):1069-77. https://doi.org/10.1007/s11517-013-1076-z.

Boling, Michelle C., Darin A. Padua, Stephen W. Marshall, Kevin Guskiewicz, Scott Pyne, and 
Anthony Beutler. 2009. "A Prospective Investigation of Biomechanical Risk Factors for Patellofemoral Pain Syndrome.” The American Journal of Sports Medicine 37 (11):210816. https://doi.org/10.1177/0363546509337934.

Boyas, S., and A. Guével. 2011. "Neuromuscular Fatigue in Healthy Muscle: Underlying Factors and Adaptation Mechanisms." Annals of Physical and Rehabilitation Medicine 54 (2):88108. https://doi.org/10.1016/j.rehab.2011.01.001.

Brown, Allison M., Rebecca A. Zifchock, Howard J. Hillstrom, Jinsup Song, and Carole A. Tucker. 2016. "The Effects of Fatigue on Lower Extremity Kinematics, Kinetics and Joint Coupling in Symptomatic Female Runners with Iliotibial Band Syndrome." Clinical Biomechanics 39 (2016). Elsevier Ltd:84-90. https://doi.org/10.1016/j.clinbiomech.2016.09.012.

Callaghan, Michael J. 2005. "Lower Body Problems and Injury in Cycling." Journal of Bodywork and Movement Therapies 9 (3):226-36. https://doi.org/10.1016/j.jbmt.2005.01.007.

Cecchini, Giulio, Gabriele Maria Lozito, Maurizio Schmid, Silvia Conforto, Francesco Riganti Fulginei, and Daniele Bibbo. 2014. "Neural Networks for Muscle Forces Prediction in Cycling." Algorithms 7 (4):621-34. https://doi.org/10.3390/a7040621.

Christian, Ryan J., David J. Bishop, François Billaut, and Olivier Girard. 2014. "The Role of Sense of Effort on Self-Selected Cycling Power Output.” Frontiers in Physiology 5 MAR (March):1-10. https://doi.org/10.3389/fphys.2014.00115.

Clarsen, Benjamin (NORWEGIAN SCHOOL OF SPORT SCIENCES). 2015. Overuse Injuries in Sport. Overuse Injuries in Sport Development, Validation and Application of a New Surveillance Method. Vol. 143.

Cote, Julie N., Denis Raymond, Pierre A. Mathieu, Anatol G. Feldman, and Mindy F. Levin. 2005. "Differences in Multi-Joint Kinematic Patterns of Repetitive Hammering in Healthy, Fatigued and Shoulder-Injured Individuals." Clinical Biomechanics 20 (6):581-90. https://doi.org/10.1016/j.clinbiomech.2005.02.012.

Craig, Neil P, and Kevin I Norton. 2001. "Characteristics of Track Cycling." Sports Medicine (Auckland, N.Z.) 31 (7):457-68. https://doi.org/10.2165/00007256-200131070-00001.

Delsys. 2014. "Trigno Wireless System User's Guide."

Dettori, Nathan J., and Daniel C. Norvell. 2006. "Non-Traumatic Bicycle Injuries: A Review of the Literature." Sports Medicine 36 (1):7-18. https://doi.org/10.2165/00007256-20063601000002.

Ellis, Richard, Wayne Hing, and Duncan Reid. 2007. "Iliotibial Band Friction Syndrome-A Systematic Review.” Manual Therapy 12 (3):200-208. https://doi.org/10.1016/j.math.2006.08.004.

Eng, Carolyn M., Allison S. Arnold, Andrew A. Biewener, and Daniel E. Lieberman. 2015. "The Human Iliotibial Band Is Specialized for Elastic Energy Storage Compared with the Chimp Fascia Lata." Journal of Experimental Biology 218 (15):2382-93. https://doi.org/10.1242/jeb.117952.

Enoka, Roger M., and Jacques Duchateau. 2016. Translating Fatigue to Human Performance. 
Medicine and Science in Sports and Exercise. Vol. 48. https://doi.org/10.1249/MSS.0000000000000929.

Fairclough, John, Koji Hayashi, Hechmi Toumi, Kathleen Lyons, Graeme Bydder, Nicola Phillips, Thomas M. Best, and Mike Benjamin. 2006. "The Functional Anatomy of the Iliotibial Band during Flexion and Extension of the Knee: Implications for Understanding Iliotibial Band Syndrome.” Journal of Anatomy 208 (3):309-16. https://doi.org/10.1111/j.1469-7580.2006.00531.x.

. 2007. "Is Iliotibial Band Syndrome Really a Friction Syndrome?" Journal of Science and Medicine in Sport 10 (2):74-76. https://doi.org/10.1016/j.jsams.2006.05.017.

Farrell, Kevin C., Kim D. Reisinger, and Mark D. Tillman. 2003. "Force and Repetition in Cycling: Possible Implications for Iliotibial Band Friction Syndrome.” Knee 10 (1):103-9. https://doi.org/10.1016/S0968-0160(02)00090-X.

Flato, Russell, Giovanni J. Passanante, Matthew R. Skalski, Dakshesh B. Patel, Eric A. White, and George R. Matcuk. 2017. "The Iliotibial Tract: Imaging, Anatomy, Injuries, and Other Pathology.” Skeletal Radiology 46 (5). Skeletal Radiology:605-22. https://doi.org/10.1007/s00256-017-2604-y.

Friedman, Michael V., J. Derek Stensby, Jeremiah R. Long, Stephen A. Currie, and Travis J. Hillen. 2017. "Beyond the Greater Trochanter: A Pictorial Review of the Pelvic Bursae." Clinical Imaging 41. Elsevier Inc.:37-41. https://doi.org/10.1016/j.clinimag.2016.09.010.

Gilbertson, Matthew. 2008. "Measuring Cycling Kinematics Using a Low-Cost, Flashing LED, Multi-Camera Approach.” Massachusetts Institute of Technology.

Godin, Jonathan A., Jorge Chahla, Gilbert Moatshe, Bradley M. Kruckeberg, Kyle J. Muckenhirn, Alexander R. Vap, Andrew G. Geeslin, and Robert F. LaPrade. 2017. “A Comprehensive Reanalysis of the Distal Iliotibial Band: Quantitative Anatomy, Radiographic Markers, and Biomechanical Properties." The American Journal of Sports Medicine 45 (11):2595-2603. https://doi.org/10.1177/0363546517707961.

Gregor, Robert J., Jeffrey P. Broker, and Mary M. Ryan. 1991. "The Biomechanics of Cycling." Exercise and Sport Science Reviews 19:127-69.

Ha, Dong Ho, Sunseob Choi, Soo Jin Kim, and Lih Wang. 2015. "Intra-Articular Fibroma of Tendon Sheath in a Knee Joint Associated with Iliotibial Band Friction Syndrome." Korean Journal of Radiology 16 (1):169-74. https://doi.org/10.3348/kjr.2015.16.1.169.

Hamill, Joseph, Ross Miller, Brian Noehren, and Irene Davis. 2008. "A Prospective Study of Iliotibial Band Strain in Runners.” Clinical Biomechanics 23 (8):1018-25. https://doi.org/10.1016/j.clinbiomech.2008.04.017.

Harman, Everett a, David J Gutekunst, Peter N Frykman, Marilyn a Sharp, Bradley C Nindl, Joseph a Alemany, and Robert P Mello. 2008. "Prediction of Simulated Battlefield Physical Performance from Field-Expedient Tests.” Military Medicine 173 (January):36-41. https://doi.org/10.7205/MILMED.173.1.36.

Hautier, Christophe Andre, Laurent Maurice Arsac, K. Deghdegh, Jimmy Souquet, Alain Belli, and Jean-Rene Lacour. 2000. "Influence of Fatigue on EMG/force Ratio and Cocontraction in Cycling." Medicine \& Science in Sports \& Exercise 32 (4):839-43. 
https://doi.org/10.1097/00005768-200004000-00017.

Heidlauf, Thomas, Thomas Klotz, Christian Rode, Ekin Altan, Christian Bleiler, Tobias Siebert, and Oliver Röhrle. 2016. "A Multi-Scale Continuum Model of Skeletal Muscle Mechanics Predicting Force Enhancement Based on Actin-titin Interaction.” Biomechanics and Modeling in Mechanobiology. Springer Berlin Heidelberg, 1-15. https://doi.org/10.1007/s10237-016-0772-7.

Hoof, Wannes Van, Koen Volkaerts, Kieran O'Sullivan, Sabine Verschueren, and Wim Dankaerts. 2012. "Comparing Lower Lumbar Kinematics in Cyclists with Low Back Pain (Flexion Pattern) versus Asymptomatic Controls - Field Study Using a Wireless Posture Monitoring System.” Manual Therapy 17 (4). Elsevier Ltd:312-17. https://doi.org/10.1016/j.math.2012.02.012.

Hopker, J. G., D. A. Coleman, H. C. Gregson, S. A. Jobson, T. Von der Haar, J. Wiles, and L. Passfield. 2013. "The Influence of Training Status, Age, and Muscle Fiber Type on Cycling Efficiency and Endurance Performance." Journal of Applied Physiology 115 (5):723-29. https://doi.org/10.1152/japplphysiol.00361.2013.

Hopkins, Chelsea, Sai Chuen Fu, Eldrich Chua, Xiaorui Hu, Christer Rolf, Ville M. Mattila, Ling Qin, Patrick Shu Hang Yung, and Kai Ming Chan. 2016. "Critical Review on the SocioEconomic Impact of Tendinopathy." Asia-Pacific Journal of Sports Medicine, Arthroscopy, Rehabilitation and Technology 4. Elsevier Ltd:9-20. https://doi.org/10.1016/j.asmart.2016.01.002.

Huang, Brady K., Juliana C. Campos, Philippe Ghobrial Michael Peschka, Michael L. Pretterklieber, Abdalla Y. Skaf, Christine B. Chung, and Mini N. Pathria. 2013. "Injury of the Gluteal Aponeurotic Fascia and Proximal Iliotibial Band: Anatomy, Pathologic Conditions, and MR Imaging." RadioGraphics 33 (5):1437-52. https://doi.org/10.1148/rg.335125171.

Hug, Francois. 2011. “Can Muscle Coordination Be Precisely Studied by Surface Electromyography?” Journal of Electromyography and Kinesiology 21 (1):1-12. https://doi.org/10.1016/j.jelekin.2010.08.009.

Jayanthi, N., C. Pinkham, L. Dugas, B. Patrick, and C. LaBella. 2013. "Sports Specialization in Young Athletes: Evidence-Based Recommendations." Sports Health: A Multidisciplinary Approach 5 (3):251-57. https://doi.org/10.1177/1941738112464626.

Ji, Qiang Ji Qiang, P Lan, and C Looney. 2006. "A Probabilistic Framework for Modeling and Real-Time Monitoring Human Fatigue." IEEE Transactions on Systems Man and Cybernetics Part A Systems and Humans 36:862-75. https://doi.org/10.1109/TSMCA.2005.855922.

Jorge, M., and M. L. Hull. 1986. "Analysis of EMG Measurements during Bicycle Pedalling." Journal of Biomechanics 19 (9):683-94. https://doi.org/10.1016/0021-9290(86)90192-2.

Kleissen, R. F M, M. C A Litjens, C. T M Baten, J. Harlaar, A. L. Hof, and G. Zilvold. 1997. "Consistency of Surface EMG Patterns Obtained during Gait from Three Laboratories Using Standardised Measurement Technique." Gait and Posture 6 (3):200-209. https://doi.org/10.1016/S0966-6362(97)01122-3.

Kos, D., E. Kerckhofs, G. Nagels, M.B. D’hooghe, and S. Ilsbroukx. 2008. 'Origin of Fatigue in 
Multiple Sclerosis: Review of the Literature." Neurorehabilitation and Neural Repair 22 (1):91-100. https://doi.org/10.1177/1545968306298934.

Kwatny, Eugene, Donald H. Thomas, and Harry G. Kwatny. 1970. “An Application of Signal Processing Techniques to the Study of Myoelectric Signals." IEEE Transactions on Biomedical Engineering BME-17 (4):303-13. https://doi.org/10.1109/TBME.1970.4502758.

Lanferdini, Fábio J., Rodrigo R. Bini, Pedro Figueiredo, Fernando Diefenthaeler, Carlos B. Mota, Anton Arndt, and Marco A. Vaz. 2016. "Differences in Pedalling Technique in Cycling: A Cluster Analysis." International Journal of Sports Physiology and Performance, no. FEBRUARY. https://doi.org/10.1123/ijspp.2015-0142.

Lisman, Peter, Francis G. O’Connor, Patricia A. Deuster, and Joseph J. Knapik. 2013. "Functional Movement Screen and Aerobic Fitness Predict Injuries in Military Training." Medicine and Science in Sports and Exercise 45 (4):636-43. https://doi.org/10.1249/MSS.0b013e31827a1c4c.

Louw, Maryke, and Clare Deary. 2014. "The Biomechanical Variables Involved in the Aetiology of Iliotibial Band Syndrome in Distance Runners - A Systematic Review of the Literature." Physical Therapy in Sport 15 (1). Elsevier Ltd:64-75. https://doi.org/10.1016/j.ptsp.2013.07.002.

Luca, Carlo J. De. 1997. “The Use of Surface Electromyography in Biomechanics.” DelSys Incorporated.

Machado, Margarida, Paulo Flores, and Benjamin J. Fregly. 2011. "A Conceptual Framework for Contact and Muscle Modeling Using OpenSim: A Prosthetic Knee Case Study.” October.

Malek, Mehrdad M, and Robert E Mangine. 1981. "Patellofemoral Pain Syndromes: A Comprehensive and Conservative Approach." Journal of Orthopaedic \& Sports Physical Therapy 2 (3):108-16.

Menard, Mathieu, Mathieu Domalain, and Patrick Lacouture. 2014. "A Musculoskeletal Modelling Approach of Iliotibial Band Syndrome in Cycling. Implications for Injury Prevention." In , 3-6.

Miller, Ross H., Jennifer L. Lowry, Stacey A. Meardon, and Jason C. Gillette. 2007. "Lower Extremity Mechanics of Iliotibial Band Syndrome during an Exhaustive Run." Gait and Posture 26 (3):407-13. https://doi.org/10.1016/j.gaitpost.2006.10.007.

National Council of Youth Sports. 2008. "Report on Trends and Participation in Organized Youth Sport," 1-19. http://www.ncys.org/pdfs/2008/2008-ncys-market-research-report.pdf.

Noakes, T. D. 2000. "Physiological Models to Understand Exercise Fatigue and the Adaptations That Predict or Enhance Athletic Performance." Scandinavian Journal of Medicine and Science in Sports 10 (3):123-45. https://doi.org/10.1034/j.1600-0838.2000.010003123.x.

Noehren, Brian, Irene Davis, and Joseph Hamill. 2007. "ASB Clinical Biomechanics Award Winner 2006. Prospective Study of the Biomechanical Factors Associated with Iliotibial Band Syndrome." Clinical Biomechanics 22 (9):951-56. https://doi.org/10.1016/j.clinbiomech.2007.07.001.

Orchard, John W. 2007. “Re: Is Iliotibial Band Syndrome a Friction Syndrome?” Journal of 
Science and Medicine in Sport 10 (2):77-78. https://doi.org/10.1016/j.jsams.2006.11.002.

Ozgünen, Kerem Tuncay, Umut Celik, and Sanlı Sadi Kurdak. 2010. "Determination of an Optimal Threshold Value for Muscle Activity Detection in EMG Analysis." Journal of Sports Science \& Medicine 9 (4):620-28.

http://www.ncbi.nlm.nih.gov/pubmed/24149789\%5Cnhttp://www.pubmedcentral.nih.gov/ar ticlerender.fcgi?artid=PMC3761824.

Öztürk, Selcen. 2013. "What Is the Economic Burden of Sports Injuries?” Joint Diseases and Related Surgery 24 (2):108-11. https://doi.org/10.5606/ehc.2013.24.

Pageaux, Benjamin. 2014. "The Psychobiological Model of Endurance Performance: An EffortBased Decision-Making Theory to Explain Self-Paced Endurance Performance." Sports Medicine (Auckland, N.Z.) 44 (9):1319-20. https://doi.org/10.1007/s40279-014-0198-2.

Petchprapa, Catherine N., and Jenny T. Bencardino. 2013. "Tendon Injuries of the Hip." Magnetic Resonance Imaging Clinics of North America 21 (1). Elsevier Inc:75-96. https://doi.org/10.1016/j.mric.2012.09.004.

Sanderson, David J. 1991. "The Influence of Cadence and Power Output on the Biomechanics of Force Application during Steady-Rate Cycling in Competitive and Recreational Cyclists." Journal of Sports Sciences 9 (2):191-203. https://doi.org/10.1080/02640419108729880.

Seijas, Roberto, Andrea Sallent, Maria Galan, Pedro Alvarez-Diaz, Oscar Ares, and Ramon Cugat. 2016. "Iliotibial Band Syndrome Following Hip Arthroscopy: An Unreported Complication.” Indian Journal of Orthopaedics 50 (5):486. https://doi.org/10.4103/00195413.189596.

Sher, Irene, Hilary Umans, Sherry A. Downie, Keith Tobin, Ritika Arora, and Todd R. Olson. 2011. "Proximal Iliotibial Band Syndrome: What Is It and Where Is It?" Skeletal Radiology 40 (12):1553-56. https://doi.org/10.1007/s00256-011-1168-5.

Sherman, Michael, Ajay Seth, and Scott Delp. 2010. "How to Compute Muscle Moment Arm Using Generalized Coordinates,” 1-12. https://doi.org/10.1115/DETC2013-13633.

So, Raymond C H, Joseph K F Ng, and Gabriel Y F Ng. 2005. "Muscle Recruitment Pattern in Cycling: A Review." Physical Therapy in Sport 6 (2):89-96. https://doi.org/10.1016/j.ptsp.2005.02.004.

Sperryn, Peter N. 1994. “Overuse Injury in Sport.” BMJ (Clinical Research Ed.) 308 (6940):1430-32. https://doi.org/10.1136/bmj.308.6940.1356.

Springer, Barbara A, and Amy E Ross. 2013. "Musculoskeletal Injuries in Military Women." https://doi.org/10.1037/e682182012-001.

Srinivasan, J., and Venkatesh Balasubramanian. 2007. "Low Back Pain and Muscle Fatigue due to Road Cycling-An sEMG Study." Journal of Bodywork and Movement Therapies 11 (3):260-66. https://doi.org/10.1016/j.jbmt.2006.08.009.

Staudenmann, Didier, Karin Roeleveld, Dick F. Stegeman, and Jaap H. van Dieen. 2010. "Methodological Aspects of SEMG Recordings for Force Estimation - A Tutorial and Review." Journal of Electromyography and Kinesiology 20 (3). Elsevier Ltd:375-87. https://doi.org/10.1016/j.jelekin.2009.08.005. 
The Physical Activity Council. 2017. "2017 Participation Report: The Physical Activity Council's Annual Study Tracking Sports, Fitness, and Recreation Participation in the US," 26. http://www.ncys.org/pdfs/2008/2008-ncys-market-research-report.pdf.

Thelen, D.G. 2003. "Adjustment of Muscle Mechanics Model Parameters to Simulate Dynamic Contractions in Older Adults." Journal of Biomechanical Engineering 125 (1):70-77.

Thelen, D.G., Ajay Seth, Frank C. Anderson, and Scott L. Delp. 2003. "OpenSim 3DGaitModel 2354.” Creative Commons (CCBY 3.0).

Tompson, Jonathan, Arjun Jain, Yann LeCun, and Christoph Bregler. 2014. "Joint Training of a Convolutional Network and a Graphical Model for Human Pose Estimation," 1-9. http://arxiv.org/abs/1406.2984.

Vestergaard, Sonja, Kushang V. Patel, Stefania Bandinelli, Luigi Ferrucci, and Jack M. Guralnik. 2009. "Characteristics of 400-Meter Walk Test Performance and Subsequent Mortality in Older Adults." Rejuvenation Research 12 (3):177-84. https://doi.org/10.1089/rej.2009.0853.

Wanich, Tony, Christopher Hodgkins, Jean-Allain Columbier, Erika Muraski, and John G Kennedy. 2007. "Cycling Injuries of the Lower Extremity." The Journal of the American Academy of Orthopaedic Surgeons 15 (12):748-56. https://doi.org/15/12/748 [pii]. 


\section{Appendix A Volunteer Characteristics}

The following tables provide a general description of the volunteer demographics, self-reported injuries, and competition/training history [Tables A-1,2,3]. Volunteers have been de-identified and coded to protect potentially identifying information.

Table A-1: Summary of Volunteer Physical Characteristics

\begin{tabular}{|c|c|}
\hline Parameter & Mean Value (Standard Error) \\
\hline Sex (M/F) & $11 \mathrm{M} / 2 \mathrm{~F}$ \\
\hline Height (in.) & $70.62(3.45)$ \\
\hline Weight (lbs.) & $167(22.15)$ \\
\hline BMI & $23.46(2.09)$ \\
\hline Age (yrs.) & $29.92(9.27)$ \\
\hline Hip-to-Heel Length (in.) & $42.75(2.31)$ \\
\hline
\end{tabular}

Table A-2: Summary of Self-Reported Prior Injuries

\begin{tabular}{|c|c|c|c|c|c|c|}
\hline $\begin{array}{c}\text { Volunteer } \\
\text { Number }\end{array}$ & $\begin{array}{c}\text { Date of } \\
\text { Most } \\
\text { Recent } \\
\text { Injury }\end{array}$ & $\begin{array}{c}\text { Leg, Foot, } \\
\text { Lower } \\
\text { Extremity? }\end{array}$ & $\begin{array}{c}\text { Shoulder, } \\
\text { Hand, } \\
\text { Upper } \\
\text { Extremity? }\end{array}$ & $\begin{array}{c}\text { Back, } \\
\text { Torso, } \\
\text { Spine? }\end{array}$ & $\begin{array}{c}\text { Head, } \\
\text { Neck? }\end{array}$ & $\begin{array}{c}\text { Treated \& } \\
\text { Cleared for } \\
\text { Activity? }\end{array}$ \\
\hline 5121 & 2016 & --- & $\bullet$ & --- & --- & Yes \\
\hline 7592 & 2010 & --- & --- & $\bullet$ & --- & Yes \\
\hline 7924 & 2003 & $\bullet$ & --- & $\bullet$ & --- & Yes \\
\hline 8437 & 2013 & - & --- & --- & --- & Yes \\
\hline 9452 & 2016 & $\bullet$ & $\bullet$ & --- & $\bullet$ & Yes \\
\hline
\end{tabular}

Remaining eight (8) volunteers had no self-reported prior injuries. 
Table A-3: Summary of Self-Reported Competition and Training History

\begin{tabular}{|c|c|c|c|c|c|c|}
\hline \multirow[b]{2}{*}{$\begin{array}{l}\text { Volunteer } \\
\text { Number }\end{array}$} & \multicolumn{5}{|c|}{ Competition Style } & \multirow[b]{2}{*}{$\begin{array}{l}\text { Training } \\
\text { Frequency? }\end{array}$} \\
\hline & College & $\begin{array}{c}\text { Long } \\
\text { Distance }\end{array}$ & Sprint & Triathlon & Mountain & \\
\hline 2222 & $-\cdots$ & $\bullet$ & $-\cdots$ & $-\cdots$ & $\bullet$ & $>2$ per week \\
\hline 2460 & --- & --- & --- & --- & --- & $\mathrm{x}$ \\
\hline 2593 & $\bullet$ & --- & $\bullet$ & --- & --- & $>2$ per week \\
\hline 2639 & --- & --- & - & - & --- & $>2$ per week \\
\hline 2786 & --- & - & --- & --- & --- & Daily \\
\hline 3579 & --- & --- & $-\cdots$ & --- & $-\cdots$ & $>2$ per week \\
\hline 4218 & --- & $\bullet$ & --- & --- & --- & $>2$ per week \\
\hline 5121 & --- & $\bullet$ & --- & --- & --- & Weekly \\
\hline 7592 & --- & - & - & • & --- & --- \\
\hline 7924 & $-\cdots$ & --- & --- & $-\cdots$ & --- & $>2$ per week \\
\hline 8105 & -- & -- & -- & -- & -- & Daily \\
\hline 8437 & $\bullet$ & --- & $\bullet$ & --- & --- & 2 per week \\
\hline 9452 & - & --- & --- & $\bullet$ & --- & $\mathrm{x}$ \\
\hline
\end{tabular}

" $\mathrm{x}$ " indicates un-reported information.

Seat and handle height settings were set using one-inch increments in the vertical direction. By the same unit seat forward/backward values increase with posterior translation. Test setup of the bike is presented in Table A-4.

Table A-4: Summary of Test Setup Characteristics

\begin{tabular}{|c|c|c|c|}
\hline \multirow{2}{*}{ Volunteer Number } & \multicolumn{3}{|c|}{ Bike Settings (in.) } \\
\cline { 2 - 4 } & Seat Height & Seat Forward/Back & Handle Height \\
\hline 2222 & $\mathrm{x}$ & 1 & 4 \\
\hline 2460 & 3 & 2 & 3 \\
\hline 2593 & 5 & 2 & 4 \\
\hline 2639 & 7 & 2 & 2 \\
\hline 2786 & 3 & 2 & 2 \\
\hline 3579 & $\mathrm{x}$ & $\mathrm{x}$ & $\mathrm{x}$ \\
\hline 4218 & $\mathrm{x}$ & 1 & 4 \\
\hline 5121 & 7 & 3 & 5 \\
\hline 7592 & 7 & $\mathrm{x}$ & 5 \\
\hline 7924 & 7 & 1 & 4 \\
\hline 8105 & 7 & 1 & 4 \\
\hline 8437 & 8 & 2 & 4 \\
\hline 9452 & 8 & & \\
\hline
\end{tabular}

" $\mathrm{x}$ " indicates un-reported information. 
Following the test, volunteer performance was categorized and comparative statistics were run on the anthropomorphic data between performance groups [Table A-5].

Table A-5: Summary of Volunteer Physical Characteristics by Performance Category

\begin{tabular}{|c|c|c|c|}
\hline \multirow{2}{*}{ Parameter } & \multicolumn{3}{|c|}{ Mean Value (Standard Error), $\mathbf{n} \mathbf{1 1}$} \\
\cline { 2 - 4 } & Non-Fatigue & Moderate Fatigue & Fatigued/Failed \\
\hline Sex (M/F) & $4 \mathrm{M} / 0 \mathrm{~F}$ & $1 \mathrm{M} / 1 \mathrm{~F}$ & $5 \mathrm{M} / 0 \mathrm{~F}$ \\
\hline Height (in.) & $70.75(3.20)$ & $67.5(2.12)$ & $72.4(2.07)$ \\
\hline Weight (lbs.) & $177.5(10.41)$ & $157.5(10.61)$ & $172.4(15.66)$ \\
\hline BMI & $24.96(1.23)$ & $24.29(0.11)$ & $23.10(1.62)$ \\
\hline Age (yrs.) & $33.5(9.33)$ & $30(12.73)$ & $30.8(9.68)$ \\
\hline Hip-to-Heel Length (in.) & $42.25(1.54)$ & $42.25(0.71)$ & $44.3(1.93)$ \\
\hline Hip-to-Head Length (in.) & $28.5(1.85)$ & $25.25(1.41)$ & $28.1(1.53)$ \\
\hline Leg Length Ratio & $0.60(0.01)$ & $0.63(0.01)$ & $0.61(0.02)$ \\
\hline
\end{tabular}




\section{Appendix B}

Coordinate Systems

\section{B.1 COORDINATE DEFINITIONS}

Following integration of the loadcells into the bike structure, local coordinate systems must be defined to provide context for measured force and moment data. The diagram below presents local coordinate system orientation and polarities with respect to loadcell placement in the pedals and seat [Figure B1].
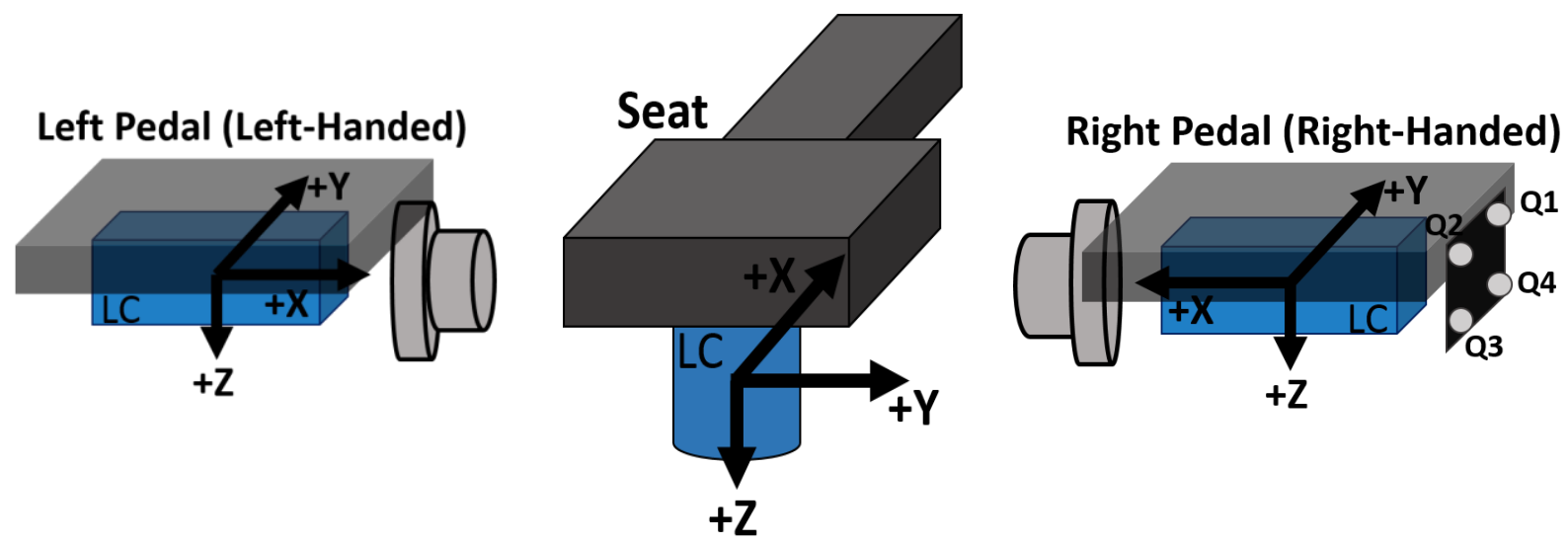

Figure B-1: Loadcell Local Coordinate System Definitions for Pedals and Seat

From the pedal motion tracking data, measured forces could be transformed to the desired coordinate system. Definition of pedal, global, and crank arm coordinates are below [Figures B2B4]. Mirroring of the pedal and crank arm coordinates was used for the left pedal coordinate systems, while global coordinate system definition remained consistent bilaterally. 


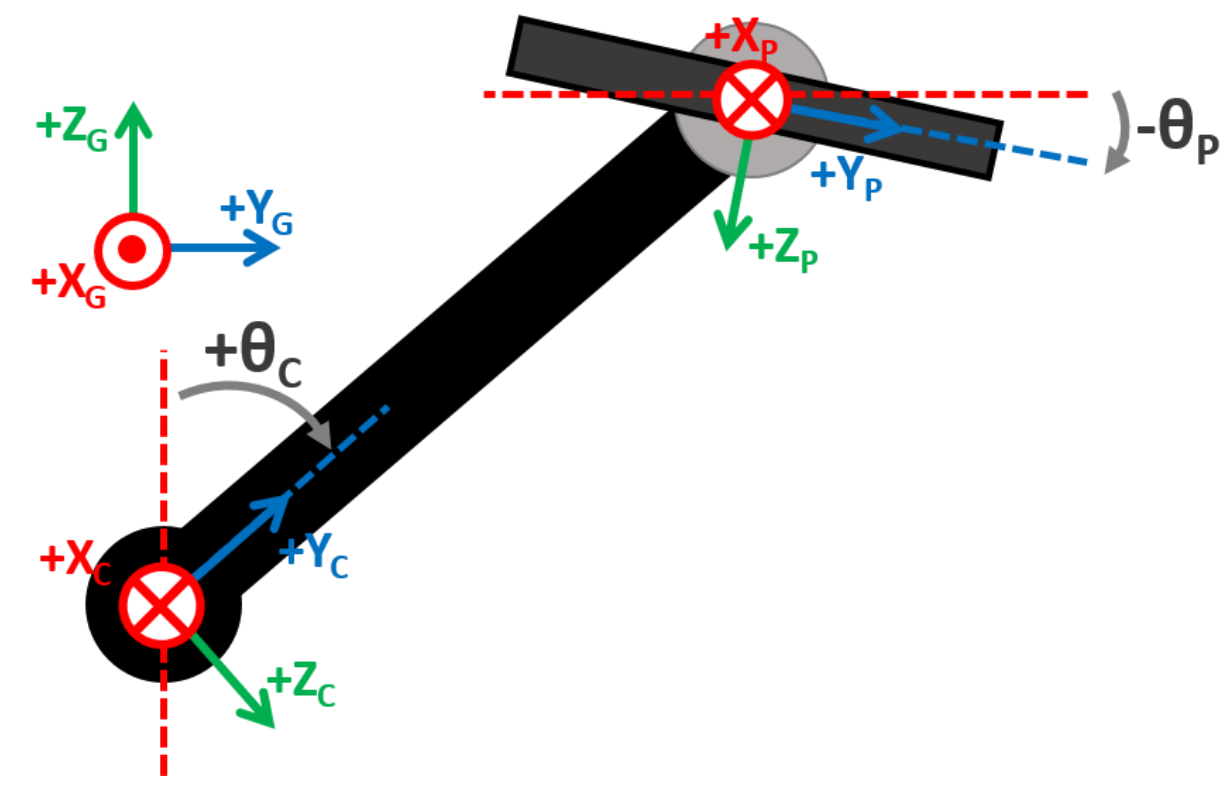

Figure B-2: Pedal, Global, and Crank Arm Coordinate System Definition for the Right Pedal

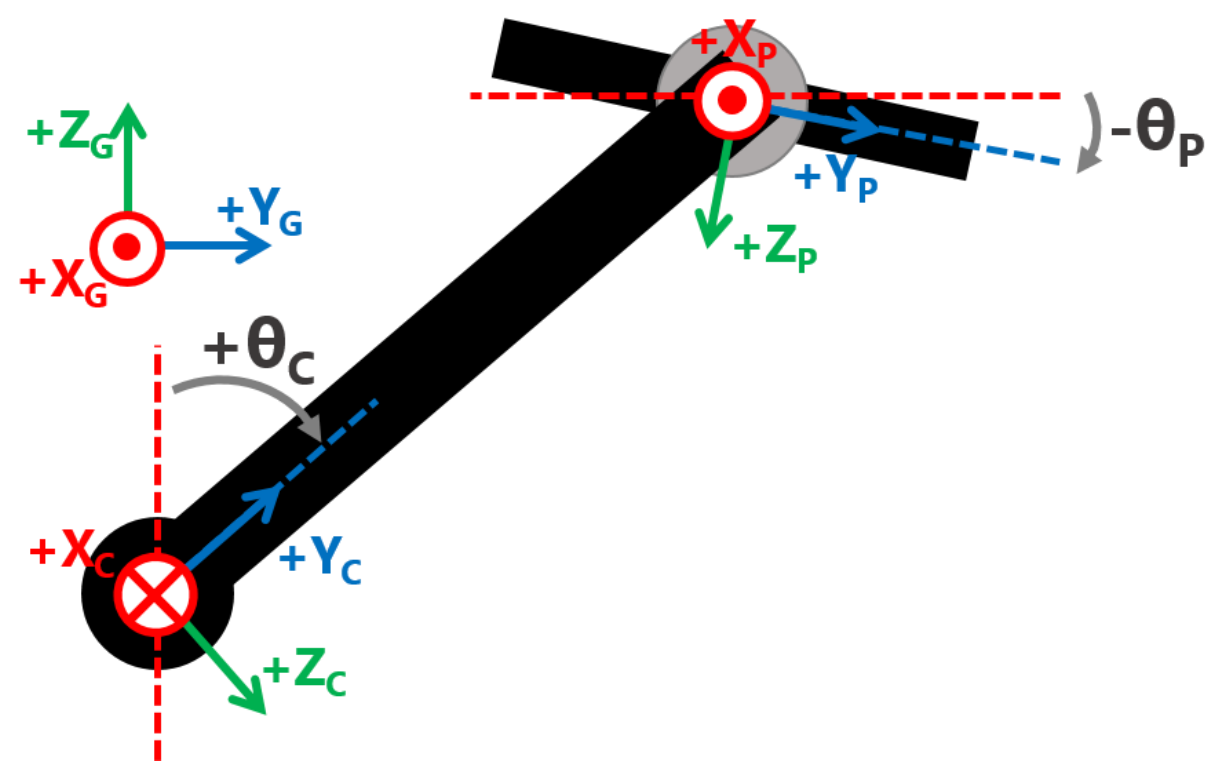

Figure B-3: Pedal, Global, and Crank Arm Coordinate System Definition for the Left Pedal 


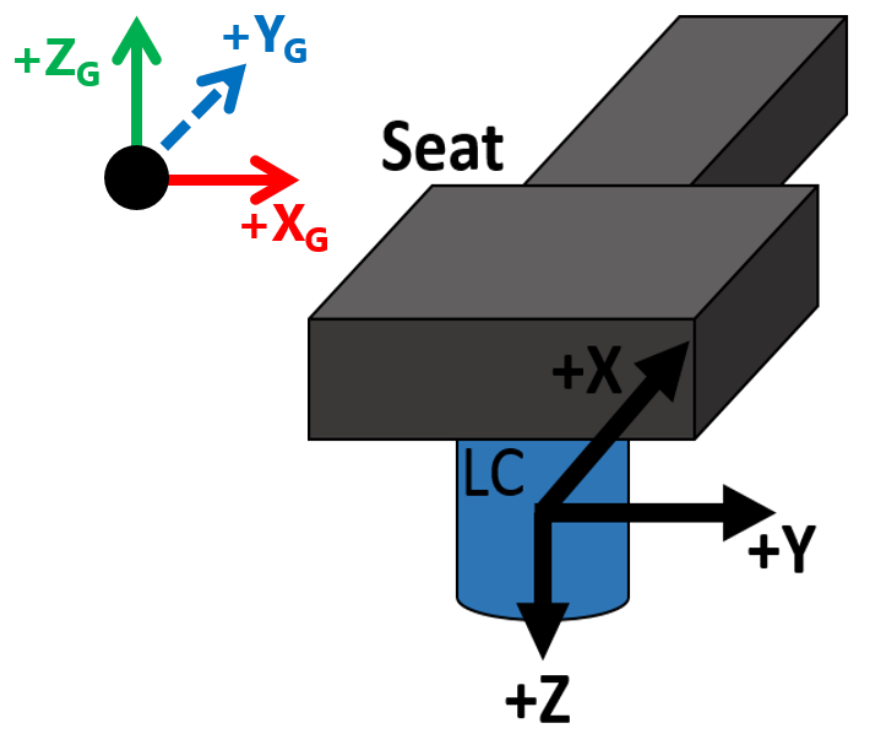

Figure B-4: Seat and Global Coordinate System Definition for the Seat

\section{B.2 COORDINATE TRANSFORMATIONS}

Vicon motion capture data from the bike pedals provided the trajectories of the pedal-mounted lateral cluster of 4 markers. Methods discussed in Chapter 4 were used to estimate pedal position, pedal orientation, and pedal loadcell location. From the pedal parameter estimates, crank arm length, orientation, and center-of-rotation could be estimated. These kinematic calculations were crucial to transforming measured pedal loads from local coordinates to global coordinates (for OpenSim modeling) and to crank arm coordinates (for power estimation).

The transformation of right loadcell forces from local pedal coordinates to global coordinates was calculated using (B1), making use of the estimated pedal orientation $\left(\boldsymbol{\theta}_{\boldsymbol{P}}\right)$ :

$$
F_{G}=\left[\begin{array}{l}
F_{G x} \\
F_{G y} \\
F_{G z}
\end{array}\right]=\left[\begin{array}{ccc}
-1 & 0 & 0 \\
0 & \cos \left(\theta_{P}\right) & \sin \left(\theta_{P}\right) \\
0 & \sin \left(\theta_{P}\right) & -\cos \left(\theta_{P}\right)
\end{array}\right]\left[\begin{array}{l}
F_{P x} \\
F_{P y} \\
F_{P z}
\end{array}\right]
$$


The transformation of right loadcell forces from global coordinates to crank arm coordinates was calculated using (B2), making use of the estimated crank arm orientation $\left(\boldsymbol{\theta}_{\boldsymbol{C}}\right)$ :

$$
F_{C}=\left[\begin{array}{l}
F_{C x} \\
F_{C y} \\
F_{C z}
\end{array}\right]=\left[\begin{array}{ccc}
-1 & 0 & 0 \\
0 & \sin \left(\theta_{C}\right) & \cos \left(\theta_{C}\right) \\
0 & \cos \left(\theta_{C}\right) & -\sin \left(\theta_{C}\right)
\end{array}\right]\left[\begin{array}{l}
F_{G x} \\
F_{G y} \\
F_{G z}
\end{array}\right]
$$

Transformations for left pedal local coordinates to global and crank arm coordinates were done (B3, B4) by removing mirroring of the x-component from the right pedal transforms.

$$
\begin{aligned}
& F_{G}=\left[\begin{array}{l}
F_{G x} \\
F_{G y} \\
F_{G z}
\end{array}\right]=\left[\begin{array}{ccc}
1 & 0 & 0 \\
0 & \cos \left(\theta_{P}\right) & \sin \left(\theta_{P}\right) \\
0 & \sin \left(\theta_{P}\right) & -\cos \left(\theta_{P}\right)
\end{array}\right]\left[\begin{array}{l}
F_{P x} \\
F_{P y} \\
F_{P z}
\end{array}\right] \\
& F_{C}=\left[\begin{array}{l}
F_{C x} \\
F_{C y} \\
F_{C z}
\end{array}\right]=\left[\begin{array}{ccc}
-1 & 0 & 0 \\
0 & \sin \left(\theta_{C}\right) & \cos \left(\theta_{C}\right) \\
0 & \cos \left(\theta_{C}\right) & -\sin \left(\theta_{C}\right)
\end{array}\right]\left[\begin{array}{l}
F_{G x} \\
F_{G y} \\
F_{G z}
\end{array}\right]
\end{aligned}
$$

Seat forces were adjusted to match polarity of OpenSim global coordinates, but the orientation of coordinate system did not need transformation (B5).

$$
F_{\text {Seat }, G}=\left[\begin{array}{l}
F_{\text {Seat }, G x} \\
F_{\text {Seat }, G y} \\
F_{\text {Seat }, G z}
\end{array}\right]=\left[\begin{array}{ccc}
0 & 1 & 0 \\
1 & 0 & 0 \\
0 & 0 & -1
\end{array}\right]\left[\begin{array}{l}
F_{S x} \\
F_{S y} \\
F_{S z}
\end{array}\right]
$$




\section{Appendix C}

\section{IRB Forms \\ Consent \& Recruitment}

The following pages present IRB paperwork, advertisements, informed-consent forms, and surveys used for volunteer engagement in this study. 


\section{HSR Protocol Cover Sheet}

HSR Submission Number: 10883

Title: Parametric Analysis of Fatigue in Stationary Biking: A Computational Approach

Committee Review Amount: Full Committee

Principal Investigator: $\quad$ Matthew Panzer, Ph.D.

(434) 296-7288

panzer@Virginia.EDU

Box: PO BOX 400746

School of Engineering \& Applied Science, Mechanical \& Aerospace

Engineering

Study Coordinator I: Austin Rivera

(609) 781-7766

atr8ec@virginia.edu

Box: 400746

Study Coordinator II: Ali Forghani Esfahani

(434) 297-8053

af5yr@virginia.edu

Box: 400746

Scientific Contact: $\quad$ Deepak Sathyanarayan

ds7rs@eservices.virginia.edu

(386)366-0547

Box:

Administrative Contact: Deepak Sathyanarayan

ds7rs@eservices.virginia.edu

(386)366-0547

Box:

Sub-Investigators:

Deepak

School of Engineering \& Applied Science, Mechanical \&

Sathyanarayan Aerospace Engineering

Sponsor(s): 1.) Double Hoo Research Grant

Protocol Number: N/A

Funding Grant(s): N/A

Five Year Update: NO

Location of Study: UVA

Multi-Site Study: NO

PRC Study: NO

GCRC Study: N/A

IND NO

IDE: NO 


\begin{tabular}{||l|l||}
\hline \multicolumn{1}{||}{ If applicable, submit one copy of any } & - Questionnaires \\
other you have such as: & $\begin{array}{l}\text { - Surveys } \\
\text { - Manual of Operations }\end{array}$ \\
& $\begin{array}{l}\text { - Package Inserts } \\
\text { - COI Management Plan }\end{array}$ \\
\hline \hline Auxiliary Documents Required for Approval: & None \\
\hline \hline Other Documents: & NONE \\
\hline
\end{tabular}

Committee Conflict: NONE 


\begin{tabular}{|c|c|c|}
\hline \multicolumn{2}{|r|}{ Question/Answers for HSR Submission: 10883} & \multirow[b]{2}{*}{ YES } \\
\hline 1. & $\begin{array}{l}\text { Are you doing research with human subjects or a clinical investigation as an agent } \\
\text { for UVa? }\end{array}$ & \\
\hline 2. & $\begin{array}{l}\text { Will the IRB-HSR be the IRB of record for this protocol for the research to be } \\
\text { done by UVa personnel? }\end{array}$ & YES \\
\hline 36. & $\begin{array}{l}\text { Do you plan to do research with data previously collected as part of an } \\
\text { Improvement Project (e.g. Performance Improvement, Practice Improvement, } \\
\text { Quality Improvement) in which there was no interaction or intervention with an } \\
\text { individual and the project only involved the use of information from UVa medical } \\
\text { records? }\end{array}$ & NO \\
\hline 38. & $\begin{array}{l}\text { Is there a protocol already in existence (e.g. sponsor's protocol, investigator } \\
\text { initiated)? }\end{array}$ & NO \\
\hline 39. & Is this a 5 year update of a previously approved protocol? & NO \\
\hline 40. & Is this protocol funded by an external grant? & NO \\
\hline 43. & $\begin{array}{l}\text { Do you or will you have a contract with an outside entity to fund this protocol OR } \\
\text { to share data with anyone not listed on the protocol, other than sponsor or CRO, } \\
\text { prior to publication? }\end{array}$ & NO \\
\hline 48. & Is there an entity inside of UVA supporting this study? & YES \\
\hline 49. & $\begin{array}{l}\text { Will this study be submitted through the PI's current prim } \\
\text { department appointment? }\end{array}$ & YES \\
\hline 50. & Is this a multi-site trial? & NO \\
\hline 51. & $\begin{array}{l}\text { Will data from this study be combined with data from other sites conducting the } \\
\text { same or similar study? }\end{array}$ & NO \\
\hline 54. & $\begin{array}{l}\text { Will any of your data involve information about students governed by the federal } \\
\text { FERPA regulations, such as information from Student Health, the Registrar's } \\
\text { Office, the Office of Assessment and Studies, or the Student Information System } \\
\text { (SIS)? }\end{array}$ & NO \\
\hline 55. & Does this study meet Exempt approval criteria? & NO \\
\hline 56. & $\begin{array}{l}\text { Will data/specimens be collected at another institution such as another health } \\
\text { system, a school or HealthSouth and sent to UVA? }\end{array}$ & NO \\
\hline 57. & Does this study meet the criteria of "non engaged" in human subject research? & NO \\
\hline 58. & Is there a conflict of interest in the protocol? & NO \\
\hline 60. & Does the study involve a patent owned by UVA? & NO \\
\hline 61. & $\begin{array}{l}\text { Will UVa release any information outside of UVa (e.g. to sponsor) about a } \\
\text { potential subject BEFORE that subject signs a consent form (e.g. screening log)? } \\
\text { NOTE: You must answer this questions YES if you are sending a screening log } \\
\text { outside of UVA (eg. sponsor) regardless of whether the screening log contains } \\
\text { HIPAA identifiers or not. }\end{array}$ & NO \\
\hline 62. & $\begin{array}{l}\text { Is there an outside funding/supply source, other than the sponsor, supporting this } \\
\text { study? }\end{array}$ & NO \\
\hline 63. & Will this study be done outside of the US and led by a research team from UVa? & NO \\
\hline 64. & $\begin{array}{l}\text { Are all personnel listed on this study volunteers working within the SOM, UVA } \\
\text { employees or UVa students? }\end{array}$ & YES \\
\hline
\end{tabular}




\begin{tabular}{|c|c|c|}
\hline 66. & $\begin{array}{l}\text { Will you do any of the following in this study? } \\
\text { - Collect or store IDENTIFIABLE* data onto ** an individual use device*** } \\
\text { consent, online surveys) via a non-UVa server. Only exception is sharing or } \\
\text { storing of data by sponsor or CRO in which data will be sent and stored in an } \\
\text { encrypted fashion (e.g. Secure FX, Secure FTP, HTTPS, PGP). } \\
\text { - Collect or store IDENTIFIABLE* data on the Cloud (e.g. UVa Box, UVa } \\
\text { Collab, Question Pro, Drop Box, Google Drive, SkyDrive, Survey Monkey etc.) } \\
\text { - Collect or store to a server NOT included in the list of HIPAA compliant } \\
\text { servers*** }\end{array}$ & NO \\
\hline 67. & $\begin{array}{l}\text { Will all of the UVA portions of this study be carried out exclusively at the UVA } \\
\text { Health System and UVA affiliated sites such as the clinics at Orange? }\end{array}$ & YES \\
\hline 68. & $\begin{array}{l}\text { To avoid conflict of interest, are any HSR board members/alternatives listed on } \\
\text { the protocol or a } 1572 \text { form as study personnel? }\end{array}$ & NO \\
\hline 69. & Will this study involve taking a family history for research purposes? & $\mathrm{NO}$ \\
\hline 70. & Will you collect data from the Clinical Data Repository (CDR)? & $\mathrm{NO}$ \\
\hline 71. & Is the ONLY intent of this protocol to establish a research database (reposi & NO \\
\hline 83. & $\begin{array}{l}\text { Does this study involve ONLY doing analysis on data collected from medical } \\
\text { records? }\end{array}$ & NO \\
\hline 86. & $\begin{array}{l}\text { Does the TARGET population of this protocol include patients with known or } \\
\text { suspected cancer? }\end{array}$ & NO \\
\hline 93. & $\begin{array}{l}\text { Is a scientific review required prior to submission of the protocol to the IRB- } \\
\text { HSR? }\end{array}$ & NO \\
\hline 94. & $\begin{array}{l}\text { Does this study involve collection of information about subjects that is not already } \\
\text { known by their health care provider and documented in their medical record AND } \\
\text { that, if released, might be damaging to an individual's financial standing, } \\
\text { employability, or reputation within the community or might lead to social } \\
\text { stigmatization or discrimination? This could include sensitive information relating } \\
\text { to sexual or gender preferences or practices; information relating to the misuse of } \\
\text { alcohol, drugs, or other addictive products; information about illegal conduct; } \\
\text { sensitive information pertaining to mental illness; information regarding HIV, } \\
\text { AIDS or other STDs; or sensitive genetic information or tissue samples. }\end{array}$ & $\mathrm{NO}$ \\
\hline 95. & $\begin{array}{l}\text { Will any data from UVA subjects be sent outside of UVA to a } \\
\text { other than the sponsor or a regulatory agency such as OHRP }\end{array}$ & NO \\
\hline 96. & Will subjects be photographed, videotaped or audiotaped? & YES \\
\hline 97. & Will this study involve only qualitative research? & NO \\
\hline 99. & Will this study involve biomedical research? & YES \\
\hline 100 & $\begin{array}{l}\text { Will this study involve an MRI with Gadolinium, in a site other than the brain, for } \\
\text { research purposes? }\end{array}$ & NO \\
\hline 101. & Does this study involve the use of medical imaging for research purposes? & NO \\
\hline 107. & Does this study involve the use of radiation therapy for research purposes? & NO \\
\hline
\end{tabular}


109. Will you be working with any specimens from a human?

116. Does this study involve the use of recombinant DNA, biological vectors or infectious agents?

117. Does this study involve a medical device that will be used at the UVa Health System that is not currently used at the UVa Health System?

118. Are you evaluating the safety and/or efficacy of a medical device in this study?

122. Are you only USING a device in an unapproved manner in this study, but NOT EVALUATING it for safety and efficacy?

123. Are you including results from DNA or RNA testing that were performed for clinical purposes using clinically validated diagnostic tests?

Will you store and maintain any specimen for unspecified future use after this

124. study is completed (specimen banking) AND/OR do any sequence analysis or other testing of the DNA or RNA as part of this research study (genetic research)?

134. Is there any possibility that any large scale human genomic data from this study would ever be shared with others outside the study team?

Does this protocol involve RESEARCH of a drug, device or biologic already

135. approved by the FDA for the indication, dose and route to be used in this protocol?

136. Does this study meet Expedited approval criteria?

Does this protocol USE or INVOLVE THE RESEARCH of a drug or biologic

137. not approved by the FDA for the indication, dose and route to be used in this protocol?

143. Will this study be monitored by a Data and Safety Monitoring Board?

144. Does this protocol involve specimen banking and/or genetic research?

166. Do you plan on getting ONLY verbal consent (Waiver of Documentation of Consent) for the entire study?

Do you plan on getting verbal consent for part of this study (Waiver of

187. Documentation of Consent) and performing another part of the study without consent of the subject (Waiver of Consent)?

208. Are you requesting that NO CONSENT (verbal or written) be obtained from the subject for the entire study (Waiver of Consent)?

209. Do you plan on getting no consent for part of the study (Waiver of Consent) and a written consent for another part of the study?

Do you plan on getting VERBAL CONSENT for ONLY a PART of this study

210. (waiver of documentation of consent) and will obtain a signature on a consent form for the main part of the study?

211. Do you plan on getting a written consent for the entire study?

212. Will you be collecting health information?

213. Will this study require separate consent forms for different parts of the protocol

213. (e.g. the screening vs. treatment portion of the protocol, or for patient vs. control)?

214. Does the study enroll adult subjects (age 18 and older)?

215. Will this study enroll subjects who would be age 65 or older? 


\begin{tabular}{|l|l|l|}
216. & Does the study enroll subjects under the age of $18 ?$ & $\mathrm{NO}$ \\
\hline 223. & Will a potential subject who is a prisoner be allowed to enroll in this study? & $\mathrm{NO}$ \\
\hline 224. & Will you enroll a woman if she is pregnant? & $\mathrm{NO}$ \\
\hline 225. & $\begin{array}{l}\text { If a woman is pregnant or becomes pregnant while in this study would the study } \\
\text { pose a risk to a fetus? }\end{array}$ & NO \\
\hline 226. & $\begin{array}{l}\text { Would participation in this study pose risks to a fetus if a male subject were to } \\
\text { father a child? }\end{array}$ & $\mathrm{NO}$ \\
\hline 227. & Will this protocol involve fetuses or human in vitro fertilization? & $\mathrm{NO}$ \\
\hline 228. & Will cognitively impaired subjects be allowed to enroll in this study? & NO \\
\hline 230. & Do you expect to enroll subjects who will not be able to read or speak English? & $\mathrm{NO}$ \\
\hline 233. & $\begin{array}{l}\text { Will the subjects receive compensation or reimbursement for expenses for } \\
\text { participating in this study? }\end{array}$ & YES \\
\hline 234. & Will this study enroll ONLY healthy subjects? & YES \\
\hline 235. & $\begin{array}{l}\text { Will this study involve RESEARCH of a drug, device, biologic or involve any } \\
\text { other intervention that might significantly affect the care of a patient? }\end{array}$ & NO \\
\hline
\end{tabular}


TEMPLATE CONSENT

\section{SECTIONS}

\begin{tabular}{|l|l||}
\hline 1. & Header (Adult) \\
\hline 2. & Header (Participant Name) \\
\hline \hline 3. & Purpose \\
\hline \hline 4. & Introduction \\
\hline \hline 5. & Procedures (Expedited) \\
\hline 6. & Procedures (Visits Duration) \\
\hline 7. & Procedures (Caveat) \\
\hline 8. & Risks \\
\hline 9. & Risks (Other Side Effects) \\
\hline 10. & Benefits \\
\hline 11. & Options (Normal Volunteers) \\
\hline 12. & Compensation \\
\hline 13. & Costs \\
\hline 14. & Injury Expedited \\
\hline 15. & Withdrawal \\
\hline 16. & Use of Information \\
\hline 17. & Study Contact Information \\
\hline 18. & HSR Contact Information \\
\hline 19. & Conclusion \\
\hline 20. & Signature (Participant) \\
\hline 21. & Signature (Person Obtaining Consent) \\
\hline 22. & Signature Impartial Witness \\
\hline & I \\
\hline
\end{tabular}

\section{PROTOCOL}

\begin{tabular}{|l|l||}
\hline 1. & Investigator Agreement \\
\hline 2. & Signature Protocol \\
\hline \hline 3. & Table of Contents \\
\hline \hline 4. & Brief Summary Abstract \\
\hline 5. & Background \\
\hline 6. & Hypothesis to be Tested \\
\hline \hline 7. & Study Design (Biomedical) \\
\hline 8. & Human Participants \\
\hline 9. & Inclusion Exclusion Criteria \\
\hline 10. & Study Design (Biomedical Statistical Analysis) \\
\hline \hline 11. & Done To Participants (Biomedical) \\
\hline 12. & Done to Participants (Taping) \\
\hline 13. & Data \& Safety Monitoring Plan (Full) Part One \\
\hline \hline & \\
\hline
\end{tabular}




\begin{tabular}{|r|l||}
\hline 14. & Data \& Safety Monitoring Plan (Full) Part Two \\
\hline 15. & Payment \\
\hline 16. & Risk Benefit Analysis \\
\hline 17. & Bibliography \\
\hline 18. & Legal Regulatory \\
\hline 19. & Recruitment \\
\hline 20. & HIPAA Criteria (with consent) \\
\hline
\end{tabular}

\section{DOCUMENT SUBMISSION FORM}

\section{DATA SECURITY PLAN}

C 2016 by the Rector and Visitors of the University of Virginia. All rights reserved. 
IRB-HSR \# 19248

Parametric Analysis of Fatigue in Stationary Biking: A Computational Approach

\section{IRB-HSR PROTOCOL}

\section{Investigator Agreement}

\section{BY SIGNING THIS DOCUMENT, THE INVESTIGATOR CONFIRMS:}

1. I am not currently debarred by the US FDA from involvement in clinical research studies.

2. I am not involved in any regulatory or misconduct litigation or investigation by the FDA.

3. That if this study involves any funding or resources from an outside source, or if you will be sharing data outside of UVA prior to publication that you will contact the Dean's office regarding the need for a contract and letter of indemnification. If it is determined that either a contract or letter of indemnification is needed, subjects cannot be enrolled until these documents are complete.

4. The proposed research project will be conducted by me or under my close supervision. It will be conducted in accordance with the protocol submitted to and approved by the IRB including any modifications, amendments or addendums submitted and approved by the IRB throughout the life of the protocol.

5. That no personnel will be allowed to work on this protocol until they have completed the IRB-HSR On-line training and the IRB-HSR has been notified.

6. That all personnel working on this protocol will follow all IRB-HSR Policies and Procedures as stated on the IRB-HSR Website http://www.virginia.edu/vprgs/irb/ and on the School of Medicine Clinical Trials Office Website:

http://knowledgelink.healthsystem.virginia.edu/intranet/hes/cto/sops/sop_index.cfm

7. I will ensure that all those delegated tasks relating to this study, whether explicitly or implicitly, are capable through expertise, training, experience or credentialing to undertake those tasks.

8. I confirm that the implications of the study have been discussed with all Departments that might be affected by it and have obtained their agreement for the study to take place.

9. That no subjects will be recruited or entered under the protocol until the Investigator has received the signed IRB-HSR Approval form stating the protocol is open to enrollment

10. That any materials used to recruit subjects will be approved by the IRB-HSR prior to use.

11. That all subjects will sign a copy of the most current consent form that has a non-expired IRB-HSR approval stamp.

12. That any modifications of the protocol or consent form will not be initiated without prior written approval from the IRB-HSR, except when necessary to eliminate immediate hazards to the subjects.

13. Any significant findings that become known in the course of the research that might affect the willingness of subjects to enroll or to continue to take part, will be promptly reported to the IRB.

14. I will report immediately to the IRB any unanticipated problems involving risk to subjects or to others including adverse reactions to biologics, drugs or medical devices.

15. That any serious deviation from the protocol will be reported promptly to the Board in writing.

Version Date: 09/06/16

Page Number: 1 
IRB-HSR \# 19248

Parametric Analysis of Fatigue in Stationary Biking: A Computational Approach

16. That any data breach will be reported to the IRB, the UVa Corporate Compliance and Privacy Office, UVa Police as applicable.

17. That the continuation status report for this protocol will be completed and returned within the time limit stated on the form.

18. That the IRB-HSR office will be notified within 30 days of a change in the Principal Investigator or of the closure of this study.

19. That a new PI will be assigned if the current PI will not be at UVA for an extended period of time. If the current PI is leaving UVa permanently, a new PI will be assigned PRIOR to the departure of the current PI.

20. All study team members will have access to the current protocol and other applicable documents such as the IRB-HSR Application, consent forms and Investigator Brochures.

21. Signed consent forms and other research records will be retained in a confidential manner. Records will be kept at least 6 years after completion of the study.

22. No data/specimens may be taken from UVa without a signed Material Transfer Agreement between OSP/SOM Grants and Contracts Office and the new institution. Original study files are considered institutional records and may not be transferred to another institution. I will notify my department administration regarding where the originals will be kept at UVa. The material transfer agreement will delineate what copies of data, health information and/or specimens may be taken outside of UVa. It will also approve which HIPAA identifiers may be taken outside of UVa with the health information or specimens.

23. If any member of study team leaves UVa, they are STRONGLY ENCOURAGED to use Exit Checklist found on IRB-HSR website at http://www.virginia.edu/provost/facultyexit.pdf.

The IRB reserves the right to terminate this study at any time if, in its opinion, (1) the risks of further experimentation are prohibitive, or (2) the above agreement is breached.

\section{Investigators Experience}

Answer/Response:

Currently involved in an IRB-approved research project using shearwave elastography on the Achilles tendons of human volunteers to determine material properties. Currently involved in multiple research projects with IRB-HSU (Human Surrogate Use) approval using human cadaver tissue for biomechanics research.

Sub-PI (GRA) and Study Coordinator will be conducting the presented research, with the assistance of qualified technicians. The PI will supervise the sub-PI, Study Coordinator, and technicians. The technicians are qualified in VICON and EMG. Technician on current study (Study Coordinator II), has a total of eight (8) years of research experience with Vicon and Delsys EMG systems through masters and Ph.D research at the McGill University, and subsequent two (2) years of work at the Center for Applied Biomechanics. Sub-PI has received training in safe Vicon and EMG use from technician. The PI routinely uses VICON data in research, and has used EMG data on occasion.

Almost all biomechanical research at the Center for Applied Biomechanics ( CAB) uses VICON, which is later used to quantify the biofidelity of human body models. EMG data was used in the past to assess neck activation levels for FE simulation of cervical spine response in automotive crash.

The sub-investigator will maintain current training in CPR and use of an AED.

Version Date: 09/06/16

Page Number: 2 of 47 
IRB-HSR \# 19248

Parametric Analysis of Fatigue in Stationary Biking: A Computational Approach

\section{Signatures}

\section{Principal Investigator}

Principal Investigator

Signature
Principal Investigator

Name Printed
Date

The Principal Investigator signature is ONLY required if this is a new protocol, a 5 year update or a modification changing the Principal Investigator.

\section{Department Chair}

BY SIGNING THIS DOCUMENT THE DEPARTMENT CHAIR AGREES:

1. To work with the investigator and with the board as needed, to maintain compliance with this agreement.

2. That the Principal Investigator is qualified to perform this study.

3. That the protocol is scientifically relevant and sound.

Department Chair or Designee

Signature

$\overline{\text { Department Chair or Designee }}$

Date

Name Printed

The person signing as the Department Chair cannot be the Principal Investigator or a subinvestigator on this protocol.

The Department Chair or Designee signature is ONLY required if this is a new protocol or a modification changing the Principal Investigator. 


\section{Brief Summary/Abstract}

As personal fitness becomes increasingly prevalent in society, a focus on maintaining safe exercise technique is crucial to avoid unintended injury. Stationary biking, or spinning, is one particular exercise that has become very popular in the past decade. Spinning is commonly done in large group settings without prior individual technique emphasis, therefore leaving the intensity and duration of spinning sessions primarily unmonitored. Anecdotal evidence of spinning-related injuries to the lower-back and knees may be attributed to improper bike setup or exercise technique. Few studies have analyzed the biomechanical consequences (both 3D kinematic and muscular) of changing whole-body spinning parameters such as CG position (body posture), pedal resistance, and pedal velocity. For these reasons, we want to investigate the question, "Can parametric analyses of stationary biking loading conditions and kinematics be used to develop a comprehensive performance and injury risk model?"

Participants in the study will be instructed to ride a stationary bike at the Center for Applied Biomechanics under pre-determined riding conditions. While riding under each condition for 20 minutes, kinematic and muscle activation data will be collected, from which a model for fatigue and performance will be developed.

The first step of gathering kinematic data from subjects biking under different postures and resistances can be efficiently done through Vicon motion tracking camera systems. In parallel with Vicon measurements, muscle activation will be recorded using six unilaterally skinmounted EMG electrodes on the gastrocnemius, hamstring group, quadriceps femoris group, gluteus maximus, erector spinae, and rectus abdominis. Combining motion capture and EMG methods with OpenSim's inverse dynamics analysis tools will allow for estimation of muscle and joint mechanical loading for each case in the parameterized behavior space, thereby computationally identifying muscle regions of high and low force output. OpenSim muscle force results can then be correlated with EMG muscle activation to give a measure of overall muscle fatigue through a force-to-activation ratio for muscle efficiency. By adding physiological measurements such as respired oxygen and heart rate, a whole-system parametric understanding of motion under the current exercise conditions can be developed.

\section{Background}

\section{Provide the scientific background, rationale and relevance of this project.}

As personal fitness becomes increasingly prevalent in society, a focus on maintaining safe exercise technique is crucial to avoid unintended injury. Stationary biking, or spinning, is one particular exercise that has become very popular in the past decade ${ }^{7}$. Spinning is commonly done in large group settings without prior individual technique emphasis, therefore leaving the intensity and duration of spinning sessions primarily unmonitored. Anecdotal evidence of spinning-related injuries to the lower-back and knees may be attributed to improper bike setup or exercise technique ${ }^{\mathbf{8}}$. Few studies have analyzed the biomechanical consequences (both 3D kinematic and muscular) of changing whole-body spinning parameters such as CG position (body posture), pedal resistance, and pedal velocity $\mathbf{2 , 3 , 5}$.

Version Date: 09/06/16

Page Number: 4 of 47 
IRB-HSR \# 19248

Parametric Analysis of Fatigue in Stationary Biking: A Computational Approach

\section{Hypothesis to be Tested}

Answer/Response:

Research Question: Can parametric analyses of stationary biking loading conditions and kinematics be used to develop a comprehensive performance and injury risk model?

Hypothesis: The investigators of this study hypothesis that statistical models of muscle activation, rider kinematics, and joint loads can be used to predict long-term injury risk. In addressing this hypothesis, the studies objectives include: 1) Collection of EMG and kinematic data from riders, 2) using kinetic and kinematic data to calculate joint loads, and 3) correlating these values to literature data on injury criteria to determine a risk of fatigue instigated injury.

\section{Will controls be used?}

\section{Study Design: Biomedical}

NO

2. What is the study design?

Observational

3. Does the study involve a placebo?

NO

\section{Human Participants}

Ages: $20-45$

Sex: $\quad M / F$

Race: Unrestricted

Subjects- see below

1. Provide target \# of subjects (at all sites) needed to complete protocol.

Answer/Response: 10 subjects

2. Describe expected rate of screen failure/ dropouts/withdrawals from all sites.

Answer/Response: 33\% (5 subjects) due dropout or incompatibility with stationary bike

3. How many subjects will be enrolled at all sites?

Answer/Response: 15 subjects

4. How many subjects will sign a consent form under this UVa protocol?

Answer/Response: 15 subjects

Version Date: 09/06/16

Page Number: 5 of 47 
Parametric Analysis of Fatigue in Stationary Biking: A Computational Approach

5. Provide an estimated time line for the study.

Answer/Response:

Subject Recruitment and Enrollment [August 2016]

Subject Testing [September-October 2016]

Data Processing [October-December 2016]

\section{Inclusion/Exclusion Criteria}

1. List the criteria for inclusion

Answer/Response:

- 2-3 years of Recreational/Competitive Biking Experience

- Inclusively between the heights of 5' 4" and 6' 6"

- Age: $20-45$ years old

\section{List the criteria for exclusion}

Answer/Response:

- Women with Known Pregnancy

- Novice/Little Biking Experience

- Children, Prisoners, Cognitively Impaired

- Non-English Speaking (To minimize potential risks due to miscommunication)

- Over 250lbs in weight

- Known heart disease (cardiomyopathy)

- Known history of arrhythmias ( irregular heart beat)

- Known current or ongoing physical injury

- History of stroke

- Hypertension ( high blood pressure)

- Any other health condition that may put a participant at risk.

3. List any restrictions on use of other drugs or treatments.

Answer/Response: No

\section{Statistical Considerations}

\section{Is stratification/randomization involved?}

Answer/Response: No

2. What are the statistical considerations for the protocol?

Answer/Response: The study is structured into three different parts: data collection, data modelling, and data analysis.

- The data collection is where the subject is put onto the stationary bike, hooked up to the EMG, is marked for motion capture and proceeds to ride until sufficient fatigue and 
raw data have been collected. The endpoint for part one is when all of the data has been collected from all of the subjects and organized to be readily available and used.

- The data modeling consists of taking the raw data collected in the first part and syncing it up with itself, i.e. having the motion capture, measured forces, and EMG muscle activation results run in parallel. The endpoint for part two is when all of the data that goes into OpenSim is able to run in parallel with the remaining data to create visual that is prepped for understanding.

- The data analysis is where the kinetics, kinematics, and dynamics of the modeling part is studied and interpreted to show a correlation between the data collected, its correlation to fatigue levels, and how this relates to risk of injury. The endpoint for part three, and the project as a whole, is when there is an understanding of the correlation between fatigue and how it promotes risk of injury.

\section{Provide a justification for the sample size used in this protocol.}

Answer/Response: Sample size was determined based off of prior literature (found in bibliography section) which used around 8 to 20 subjects max for similar experiments and successfully reach significant conclusions. There are also restrictions in funds which keep the amount of subjects to a maximum of around 15 .

\section{What is your plan for primary variable analysis?}

Answer/Response: We plan on obtaining the force, motion capture, and EMG readings for all of our subjects. Through regression, cross-correlation, and time-series machine learning analysis, a number of post-processing variables will be calculated and compared during the fatigue process. Examples of variables to be calculated from the raw data include: pedal force symmetry, seat load balance, joint angular velocities, applied-torque estimation, muscle activation symmetry, and nerve conduction velocity. By comparing the trends in these parameters over time, an understanding of fatigue-induced biking behavior can be developed.

\section{What is your plan for secondary variable analysis?}

Answer/Response: The secondary variable analysis is entails interpretation of the data collected from the primary study and OpenSim model development. OpenSim allows from collected motion and force data to be put into a volunteer-scaled simulation to approximate the muscle and joint forces for the lower extremities and torso. OpenSim also outputs predicted muscle activation levels, which will be cross-referenced with the collected EMG data. Muscle and joint force predictions from OpenSim will be incorporated into the primary variable analysis of correlations between the rider parameters. Correlations between the fatigue-induced parameter changes and locations of increased joint or muscle forces will allow for the development of probabilistic long-term injury risk estimation models.

\section{Have you been working with a statistician in designing this protocol? Answer/Response: No}

IF YES, what is their name?

Answer/Response: N/A

Version Date: 09/06/16

Page Number: 7 of 47 
IRB-HSR \# 19248

Parametric Analysis of Fatigue in Stationary Biking: A Computational Approach

7. Will data from multiple sites be combined during analysis?

Answer/Response: No

\section{Biomedical Research}

\section{What will be done in this protocol?}

Answer/Response:

1. Provide subject with proper attire and privacy to change.

2. Instruct subject to follow standard stretching protocol to limber up, and then stretch in any way they prefer to feel comfortable going for a long bike ride.
a. 15 seconds of static standing quadriceps stretch
b. 15 seconds of static toe touching with one foot over the other (each side)
c. 15 seconds of static side lunges (each side)
d. 10 active forward walking lunges (each side)
e. 10 seconds of static shoulder stretching (each side)
f. 20 seconds of static sitting straddle
g. 15 seconds of static pigeon stretch or preferred equivalent stretch (each side)
h. 15 seconds of static calf stretch (each side)
i. 5 minutes of monitored personal stretching

3. Hydrate volunteer with $8 \mathrm{oz}$. of water.

4. Mount subject onto the stationary bike.

5. Confirm adjustments made to the bike are comfortable for the rider, and following these guidelines:

Seat Height

- Start leveled with hip bone

- Slight adjustments can be made for subject's comfort

- Sitting on the seat with heel on the pedal (6 o'clock)

$\circ$ Full Leg Extension

- $\quad$ Sitting on the seat with balls of your feet on the pedal (6 o'clock)

$\circ 1^{145^{\circ}}-155^{\circ}$ Leg Extension

Handle Bar Adjustments

- Start leveled with the seat

- Slight adjustments can be made up or down for subject's proper weight distribution and comfort

Handle Bar to Seat

Version Date: 09/06/16

Page Number: 8 of 47 
IRB-HSR \# 19248

Parametric Analysis of Fatigue in Stationary Biking: A Computational Approach

- Elbow to second knuckles (proximal interphalangeal joints) should be the distance between the edge of the seat and the handle bar stand

- Slight Adjustments can be made to relieve any discomfort

6. Warm-up ride
a. 2 minutes, low resistance, speed between 100 and $110 \mathrm{rpm}$
b. 1 minute, intermediate resistance,
c. 2 minutes, low resistance, speed between 90 and $100 \mathrm{rpm}$ speed between 100 and $110 \mathrm{rpm}$

7. Hydrate volunteer with $8 \mathrm{oz}$. of water.

8. Prompt for additional stretching or adjustments if needed

a. Confirm of any discomforts

b. Adjust bike if needed

c. Require additionally stretching if needed

9. Attach twelve EMG electrodes to subject's legs, lower back, and abdominal muscle groups using proper adhesive pads.Subject to shave hair from area of electrode patch placement if required.

10. Attach Vicon markers throughout subject's body for motion capture.

11. Endurance Routine (Stationary Bike)

a. 2.5 minutes, low resistance,

speed between 60 and $75 \mathrm{rpm}$

b. 2.5 minutes, intermediate resistance, speed between 60 and $75 \mathrm{rpm}$

c. 2.5 minutes, high resistance, speed between 60 and $75 \mathrm{rpm}$

d. Maintain**, high resistance, speed between 60 and $75 \mathrm{rpm}$ **Attempt to maintain 60-75rpm without dropping below 30rpm

12. Clean-up \& Sterilization

a. Provide towels for subject to remove excess sweat

b. Provide light snacks and refreshments to subject

c. Disinfect bike with Cavicide or appropriate reagent

13. Data Collection \& Storage

a. EMG muscle activation data is collected for each muscle group being studied via the attached electrodes

b. Vicon motion capture kinematics data is being collected from each angle the cameras are set up to track the bodies movement

2. List the procedures, in bullet form, that will be done for RESEARCH PURPOSES as stipulated in this protocol.

Answer/Response: all

Version Date: 09/06/16

Page Number: 9 of 47 
IRB-HSR \# 19248

Parametric Analysis of Fatigue in Stationary Biking: A Computational Approach

3. Will you be using data/specimens in this study that were collected previously, with the use of a research consent form, from another research study?

Answer/Response: No

4. Will any of the procedures listed in item \# 2 have the potential to identify an incidental finding? This includes ALL procedures, assessments and evaluations that are being done for RESEARCH PURPOSES that may or may not be considered investigational.

Answer/Response: No

IF YES, check one of the following two options:

N/A__ The examination(s) utilize(s) the same techniques, equipment, etc., that

would be used if the subject were to have the examination(s) performed for clinical

care. There exists the potential for the discovery of clinically significant incidental findings.

- The PI takes full responsibility for the identification of incidental findings:

- The PI will inform the subjects verbally of all incidental findings that are of clinical significance or are of questionable significance.

- A follow-up letter describing the finding should be provided to the subject with instructions to either show the letter to their PC or if the subject has no PCP, the subject should be instructed to make an appointment at UVa or at the Free Clinic.

N/A_This examination(s) utilizes non-standard/investigational, technique, equipment, etc. It is impossible to determine the significance of such results, therefore abnormalities will not be shared with the subject because the meaning of the exam is not yet proven and is of unknown clinical benefit.

5. Do any of the procedures listed above, under question \# 2, utilize any imaging procedures for RESEARCH PURPOSES?

Answer/Response: No

IF YES, list procedures:

Answer/Response: N/A

IF YES, check one of the following two options:

N/A_ This imaging research examination utilizes the same imaging techniques, equipment, scanning sequences that would be used if the subject were to have the imaging performed for clinical care. There exists the potential for the discovery of clinically significant incidental findings.

If checked, answer the following:

Version Date: 09/06/16

Page Number: 10 of 47 
Parametric Analysis of Fatigue in Stationary Biking: A Computational Approach

Will the images be read by a licensed radiologist and the reading placed in the subject's medical record?

Answer/Response: N/A

-IF NO: The PI takes full responsibility for the identification of incidental findings:

- The PI will have all incidental findings reviewed by a radiologist who will advise the PI regarding clinical significance.

- The PI will inform the subjects verbally of all incidental findings that are of clinical significance or are of questionable significance.

- A follow-up letter describing the finding should be provided to the subject with instructions to either show the letter to their PC or if the subject has no PCP, the subject should be instructed to make an appointment at UVa or at the Free Clinic.

N/A_ This imaging research examination utilizes non-standard/investigational imaging modality, techniques, equipment, scanning sequences, etc. It is impossible to determine the significance of such images, therefore abnormalities will not be shared with the subject because the meaning of the exam is not yet proven and is of unknown clinical benefit.

6. Will you be using viable embryos?

IF YES, attach approval from UVA ESCRO Committee

Answer/Response: No

7. Will you be using embryonic stem cells?

IF YES, attach approval from UVA ESCRO Committee

Answer/Response: No

8. Are any aspects of the study kept secret from the participants?

Answer/Response: No

IF YES, describe:

Answer/Response: N/A

9. Is any deception used in the study?

Answer/Response: No

IF YES, describe:

Answer/Response: N/A

10. If this protocol involves study treatment, explain how a subject will be transitioned from study treatment when they have completed their participation in the study.

Version Date: 09/06/16

Page Number: 11 of 47 
IRB-HSR \# 19248

Parametric Analysis of Fatigue in Stationary Biking: A Computational Approach

Example: If the subject will be taking an investigational drug, will they need to be put back on an approved drug when they have completed the study? If yes, explain how this will be accomplished and who will cover the cost. If the subject has a device implanted will it be removed? Again- who will cover the cost of the removal?

Instructions: Answer NA if this study does not involve a study treatment.

Answer/Response: N/A

11. Will your study involve measures (C-SSRS/BID/SCID etc.) used to assess for depression and/or suicidality for research purposes?

Answer/Response: No

\section{Answer this question YES if any of the following apply:}

1) The protocol has a research purpose to study suicide, suicidal ideation, depression or trauma

2) The protocol has a research purpose to study traumatic life events that may evoke powerful emotion or induce mood changes in participants;

3) The protocol includes assessments (e.g. Surveys, exams, questionnaires, etc.) that can be used to identify depression and/or suicidal ideation (thoughts of suicide, either active or passive), plan (the means or mechanism) or intent (the expressed desire and willingness to act on the plan).

\section{IF YES: N/A}

a. Which research staff members are qualified to assess suicidality/depression and will be available to provide care and intervention?

Answer/Response: N/A

b. Include specific guidelines for intervention based on assessment tools and rating scales. (i.e. based on C-SSRS/BID, SCID score of xxx, subject will be assessed further by the PI for suicide risk or referred urgently to an ED, crisis center, or clinic immediately). Answer/Response: N/A

c. Describe a plan to link participants to psychological help if needed and include written materials listing those resources as an attachment to the protocol. This plan should include details of the planned interventions for differing severities of depression or suicidality, including a plan for how imminent risk of harm will be handled for the study's targeted population. (May include a list of local psychiatry/psychotherapy providers at UVA). Note: If the subject is a patient at UVA Medical Center, you must adhere to Medical Center Policy 0140 Judicial Treatment Order and 0197 Suicide Risk Assessment and Prevention.

Answer/Response: N/A

Version Date: 09/06/16

Page Number: 12 of 47 
IRB-HSR \# 19248

Parametric Analysis of Fatigue in Stationary Biking: A Computational Approach

d. Describe a plan to address the situation if a participant is assessed to be a danger to themselves, but refuses treatment. (the plan may include steps to contact 911) Note: If the subject is a patient at UVA Medical Center, you must adhere to you must adhere to Medical Center Policy 0140 Judicial Treatment Order and 0197 Suicide Risk Assessment and Prevention.

Answer/Response: N/A

e. Will subjects, who discontinue or are withdrawn secondary to suicidal ideations/depression prior to study completion, be asked to come to the site for an early withdrawal visit as soon as possible?

Answer/Response: N/A

If No, provide outline of plan for follow-up or indicate if follow up is not required.

Answer/Response: N/A

12. Where will the study procedures be done?

Check One:

UVA medical center facilities ( In patient or outpatient)

X_ UVa, but not medical center facilities: LIST specific location

Answer/Response: North Fork Research Park

Other LIST specific location Answer/Response:

13. If the study involves medical risk and study procedures will be done outside of the UVa Medical Center what is your plan to protect the subjects in case of a medical emergency? Check all applicable options:

$\mathrm{MD}, \mathrm{RN}$, onsite during procedures

$\mathrm{X}$ Individual trained in CPR on site during procedures

$X \quad A E D$ and Individual trained to use it onsite

$\mathrm{X}$ Call 911

Other: Describe Answer/Response:

Details for Emergency Response Time Estimation:

Rescue Stations: \#12 Hollymead (5min from CAB), \#8 Berkmar (12min from CAB) Emergency Care:

- Martha Jefferson Outpatient Care Center (5min from CAB)

- UVA Hospital or Martha Jefferson Hospital (25min from CAB)

\section{Taping/Photography}

1. Will participants be recorded on audiotape?

Answer/Response: No

IF YES, answer the following questions.

1a. What steps will be taken to protect the privacy of the subjects?

Version Date: 09/06/16

Page Number: 13 of 47 
IRB-HSR \# 19248

Parametric Analysis of Fatigue in Stationary Biking: A Computational Approach

Answer/Response: N/A

1b. What data will be captured from the audiotapes?

Answer/Response: N/A

1c. When will data from the tapes be transcribed?

Answer/Response: N/A

1d. When will the audiotapes be destroyed?

Answer/Response: N/A

2. Will participants be photographed or recorded on videotape?

Answer/Response: Yes

IF YES, answer the following questions.

2a. Will their faces be shown?

Answer/Response: Yes

2b. What steps will be taken to protect the privacy of the subjects? Answer/Response:

- Video Storage on Secure Internal UVA Computers

- Blocking/Blurring Faces if Intended for Public Release

2c. What data will be captured from the photo or videotape that could not be obtained in other ways?

Answer/Response:

- Motion Tracking and Measurement Validation

2d. How is this data critical to this research?

Answer/Response:

- Allows Motion Tracking Data Accuracy to be Reaffirmed Visually

2e. When will data from the tapes be transcribed?

Answer/Response:

- Data from tapes will not be transcribed (No Audio Recorded)

2f. When will the tapes be destroyed?

Answer/Response:

- Tapes will be kept indefinitely on secure CAB owned devices.

2g. Will participants be photographed, recorded or videotaped without their knowledge?

Answer/Response: No

Version Date: 09/06/16

Page Number: 14 of 47 
IRB-HSR \# 19248

Parametric Analysis of Fatigue in Stationary Biking: A Computational Approach

3. If a subject withdraws from the study how will you withdraw them from the audiotape, videotape or photograph?

Answer/Response: All data collected/recoded from subjects who choose to withdraw will be deleted from all storage devices.

INSTRUCTIONS: If you are collecting or storing digital images on an individual use device such as a camera, video recorder, remember to include the use of this individual use device in the Data Security Plan. 
IRB-HSR \# 19248

Parametric Analysis of Fatigue in Stationary Biking: A Computational Approach

\section{Data and Safety Monitoring Plan}

INSTRUCTIONS:

If you have any questions completing this section call 243-9847 for assistance.

A Sponsor is defined as entity that will receive data prior to publication.

\section{Definition:}

1.1 How will you define adverse events (AE)) for this study?

\section{Check all that apply}

$\underline{X}$ An adverse event will be considered any undesirable sign, symptom or medical or psychological condition even if the event is not considered to be related to the investigational drug/device/intervention. Medical condition/diseases present before starting the investigational drug/intervention will be considered adverse events only if they worsen after starting study treatment/intervention. An adverse event is also any undesirable and unintended effect of research occurring in human subjects as a result of the collection of identifiable private information under the research. Adverse events also include any problems associated with the use of an investigational device that adversely affects the rights, safety or welfare of subjects.

Will use definitions provided in the non IRB Protocol (Sponsor's, Investigator-Initiated, CTEP etc.)

Other: Specify Answer/Response:

\subsection{How will you define serious adverse events?}

Check all that apply

$\mathrm{X} \quad$ A serious adverse event will be considered any undesirable sign, symptom, or medical condition which is fatal, is life-threatening, requires or prolongs inpatient hospitalization, results in persistent or significant disability/incapacity, constitutes a congenital anomaly or birth defect, is medically significant and which the investigator regards as serious based on appropriate medical judgment. An important medical event is any AE that may not result in death, be life-threatening, or require hospitalization but may be considered an SAE when, based upon appropriate medical judgment, it may jeopardize the patient and may require medical or surgical intervention to prevent one of the outcomes listed in the definitions of SAEs.

$\mathrm{X} \quad$ Any serious psychological and emotional distress resulting from study participation (suggesting need for professional counseling or intervention).

Will use definitions provided in the non IRB Protocol (Sponsor's, Investigator-Initiated, CTEP etc.)

Version Date: 09/06/16

Page Number: 16 of 47 
Other: Specify Answer/Response:

\subsection{What is the definition of an unanticipated problem?}

Do not change this answer

An unanticipated problem is any event, experience that meets ALL 3 criteria below:

- Is unexpected in terms of nature, severity or frequency given the research procedures that are described in the protocol-related documents AND in the characteristics of the subject population being studies

- Related or possibly related to participation in research. This means that there is a reasonable possibility that the incident may have been caused by the procedures involved in the research study.

- The incident suggests that the research placed the subject or others at greater risk of harm than was previously known or recognized OR results in actual harm to the subject or others

\subsection{What are the definitions of a protocol violation and/or noncompliance?}

Do not change this answer

A protocol violation is defined as any change, deviation, or departure from the study design or procedures of research project that is NOT approved by the IRB-HSR prior to its initiation or implementation. Protocol violations may be major or minor violations.

Noncompliance can be a protocol violation OR deviation from standard operating procedures, Good Clinical Practices (GCPs), federal, state or local regulations.

Noncompliance may be serious or continuing.

Additional Information: see the IRB-HSR website at http://www.virginia.edu/vpr/irb/HSR_docs/Forms/Protocol_Violations_\%20Enrollm ent_Exceptions_Instructions.doc

\subsection{If pregnancy occurs how will this information be managed?}

$\mathrm{X} \quad$ Adverse Event- will follow adverse event recording and reporting procedures outlined in section 3 .

Unanticipated Problems- will follow Unanticipated Problem recording and reporting procedures outlined in section 3 .

Other Specify Answer/Response:

\subsection{What is the definition of a Protocol Enrollment Exception?}

$X \quad N A-$ No outside sponsor

Version Date: 09/06/16

Page Number: 17 of 47 
IRB-HSR \# 19248

Parametric Analysis of Fatigue in Stationary Biking: A Computational Approach

Protocol has a sponsor or a Data \& Safety Monitoring Board (DSMB) outside of UVa. An enrollment exception is the DSMB or sponsor's prospective approval for the enrollment of a research subject that fails to meet current IRB-HSR approved protocol inclusion criteria, or falls under protocol exclusion criteria. Enrollment exceptions only apply to a single individual. Such a request should be rare and justified in terms of serving the best interests of the potential study participant.

\subsection{What is the definition of a data breach?}

Do not change this answer

A data breach is defined in the HITECH Act (43 USC 17932) as an unauthorized acquisition, access, or use of protected health information ( $\mathrm{PHI}$ ) that compromises the security or privacy of such information.

Additional Information may be found on the IRB-HSR Website: Data Breach

\section{Identified risks and plans to minimize risk}

\subsection{What risks are expected due to the intervention in this protocol?}

Expected: is identified in nature, severity or frequency in the study documentation (protocol, consent, Investigator Brochure, package insert etc.) is considered an expected.

\begin{tabular}{|c|c|}
\hline $\begin{array}{l}\text { Expected Risks related to study } \\
\text { participation. }\end{array}$ & Frequency \\
\hline Excess Exhaustion & $\begin{array}{l}\text { Occurs frequently } \\
\mathrm{X} \text { Occurs infrequently } \\
\text { Occurs rarely } \\
\text { Frequency unknown }\end{array}$ \\
\hline $\begin{array}{l}\text { Joint Pain } \\
\text { (Knee/Back) }\end{array}$ & $\begin{array}{c}\text { Occurs frequently } \\
\text { Occurs infrequently } \\
\mathrm{X} \text { Occurs rarely } \\
\text { Frequency unknown }\end{array}$ \\
\hline Excess Dehydration & $\begin{array}{l}\text { Occurs frequently } \\
\text { X_Occurs infrequently } \\
\text { Occurs rarely } \\
\text { Frequency unknown }\end{array}$ \\
\hline Muscle Ache/Fatigue & $\begin{array}{ll}\mathrm{X} \quad \text { Occurs frequently } \\
\text { Occurs infrequently } \\
\text { Occurs rarely } \\
\text { Frequency unknown }\end{array}$ \\
\hline Fall from Bike & $\begin{array}{l}\text { Occurs frequently } \\
\text { Occurs infrequently } \\
\mathrm{X} \quad \text { Occurs rarely } \\
\\
\text { Frequency unknown }\end{array}$ \\
\hline
\end{tabular}

Version Date: 09/06/16

Page Number: 18 of 47 
IRB-HSR \# 19248

Parametric Analysis of Fatigue in Stationary Biking: A Computational Approach

\begin{tabular}{|l|l|}
\hline \multicolumn{1}{|c|}{$\begin{array}{l}\text { Minor skin irritation/abrasion from } \\
\text { shaving hair off skin }\end{array}$} & - Occurs frequently \\
& - Occurs infrequently \\
& - Frequency unknown \\
\hline $\begin{array}{l}\text { Violation of subject's privacy and } \\
\text { confidentiality }\end{array}$ & $\begin{array}{l}\text { Minimized due to the requirements of } \\
\text { the privacy plan in this protocol }\end{array}$ \\
\hline
\end{tabular}

2.2 List by bullet format a summary of safety tests/procedures/observations to be performed that will minimize risks to participants:

- Excess Exhaustion: Observation and compliance with volunteer verbal feedback

- Excess Dehydration: Observation and compliance with volunteer verbal feedback

- Joint Pain: Observation and compliance with volunteer verbal feedback

- Muscle Ache/Fatigue: Provide access to ice packs after testing

- Fall from Bike: Assistance provided when mounting and dismounting bike

2.3 Under what criteria would an INDIVIDUAL SUBJECT'S study treatment or study participation be stopped or modified

$\underline{\mathrm{X}} \quad$ At subject, $\mathrm{PI}$ or sponsor's request

$\mathrm{X}$ Treatment would be stopped if the subject had a serious adverse event deemed related to study,

Refer to the non- IRB Protocol (Sponsor's, Investigator-Initiated, CTEP protocol etc.

2.4 Under what criteria would THE ENTIRE STUDY need to be stopped.

X Per IRB, PI,

Refer to the non- IRB Protocol (Sponsor's, Investigator Initiated protocol etc.)

Other: Specify Answer/Response:

2.5 What are the criteria for breaking the blind/mask?

X NA - Not blinded/masked

Refer to the non- IRB Protocol (Sponsor's, Investigator Initiated protocol etc.)

Other: Specify Answer/Response:

2.6 How will subject withdrawals/dropouts be reported to the IRB prior to study completion?

X IRB-HSR continuation status form

Other: Specify Answer/Response:

Version Date: 09/06/16

Page Number: 19 of 47 
IRB-HSR \# 19248

Parametric Analysis of Fatigue in Stationary Biking: A Computational Approach

\section{Adverse Event / Unanticipated Problem Recording and Reporting}

3.1 Will all adverse events, as defined in section 1.1, be collected/recorded? Answer/Response: no

-IF NO, what criteria will be used?

__ Only adverse events deemed related/possibly related to study

Only adverse events that are deemed serious

$-x$ Only adverse events that are deemed related AND serious

If protocol requires oversight by Cancer Center DSMC and the protocol is Investigator Initiated- will use tables from Question \#8 to document what will be recorded.

If unsure if Cancer Center DSMC will have oversight see Question \#6 for guidance.)

Other: Specify Answer/Response:

3.2 How will adverse event data be collected/recorded? Check all that apply Paper AE forms/source documents

X Spreadsheet: paper or electronic Database Specify name/type of database Answer/Response:

3.3. How will AEs be classified/graded? Check all that apply

World Health Organization Criteria (WHO)

$\mathrm{NCl}$ Common Toxicity Criteria, Version 2.0/ NCl Common Terminology Criteria, Version 3.0

$\mathrm{NCI}$ CTCAE Version 4.0

X Mild/Moderate/Severe

X Serious/Not serious Required for all protocols

Will use classifications/grades provided in the non IRB Protocol (Sponsor's, Investigator-Initiated, CTEP etc.)

Other: Specify Answer/Response:

3.4 What scale will the PI use when evaluating the relatedness of adverse events to the study participation? Check all that apply.

_ $\mathrm{X}$ The PI will determine the relationship of adverse events to the study using the following scale:

Version Date: 09/06/16

Page Number: 20 of 47 
IRB-HSR \# 19248

Parametric Analysis of Fatigue in Stationary Biking: A Computational Approach

Related: $\quad A E$ is clearly related to the intervention

Possibly related: $\quad A E$ may be related to the intervention

Unrelated:

$A E$ is clearly not related to intervention

Will use attribution scale provided in the non IRB Protocol (Sponsor's, Investigator-Initiated, CTEP etc.)

The PI will use an alternative attribution scale. Specify Answer/Response:

\subsection{When will recording/reporting of adverse events/unanticipated problems begin?}

After subject signs consent

$\mathrm{x}$

After subject begins study intervention

Other: Specify Answer/Response:

3.6 When will the recording/reporting of adverse events/unanticipated problems end?

$\underline{X}$ End of study intervention/participation

30 days post study drug/device/intervention

Subject completes intervention and follow up period of protocol

Other: Specify Answer/Response:

See non IRB Protocol (Sponsor's, Investigator-Initiated, CTEP et.) for additional information.

Two years past last exposure to Gadolinium ONLY if diagnosed with NSF/NSD.

This must be checked if protocol involves administration of Gadolinium for

research purposes

3.7 How will Adverse Events, Unanticipated Problems, Protocol Violations and Data Breaches be reported? Complete the table below to answer this question

\begin{tabular}{|l|l|l|l|}
\hline Type of Event & $\begin{array}{c}\text { To whom will it } \\
\text { be reported: }\end{array}$ & $\begin{array}{l}\text { Time Frame for } \\
\text { Reporting }\end{array}$ & How reported? \\
\hline $\begin{array}{l}\text { Any internal event resulting } \\
\text { in death that is deemed } \\
\text { DEFINITELY related to (caused } \\
\text { by) study participation } \\
\begin{array}{l}\text { An internal event is one that } \\
\text { occurs in a subject enrolled in } \\
\text { a UVa protocol }\end{array}\end{array}$ & IRB-HSR & Within 24 hours & IRB Online and phone call \\
\hline $\begin{array}{l}\text { Internal, Serious, Unexpected } \\
\text { related adverse event }\end{array}$ & IRB-HSR & $\begin{array}{l}\text { Within 7 calendar } \\
\text { days from the } \\
\text { time the study } \\
\text { team received } \\
\text { knowledge of the }\end{array}$ & IRB Online \\
\hline
\end{tabular}

Version Date: 09/06/16

Page Number: 21 of 47 
IRB-HSR \# 19248

Parametric Analysis of Fatigue in Stationary Biking: A Computational Approach

\begin{tabular}{|c|c|c|c|}
\hline & & $\begin{array}{l}\text { event. } \\
\text { Timeline includes } \\
\text { submission of } \\
\text { signed hardcopy } \\
\text { of AE form. }\end{array}$ & \\
\hline $\begin{array}{l}\text { Unanticipated Problems that } \\
\text { are not adverse events or } \\
\text { protocol violations } \\
\text { This would include a Data } \\
\text { Breach. }\end{array}$ & IRB-HSR & $\begin{array}{l}\text { Within } 7 \text { calendar } \\
\text { days from the } \\
\text { time the study } \\
\text { team received } \\
\text { knowledge of the } \\
\text { event. }\end{array}$ & $\begin{array}{l}\text { Unanticipated Problem report } \\
\text { form. } \\
\text { http://www.virginia.edu/vprgs } \\
\text { /irb/HSR_docs/Forms/Reportin } \\
\text { g_Requirements- } \\
\text { Unanticipated_Problems.doc) }\end{array}$ \\
\hline $\begin{array}{l}\text { Protocol } \\
\text { Violations/Noncompliance } \\
\text { The IRB-HSR only requires that } \\
\text { MAJOR violation be reported, } \\
\text { unless otherwise required by } \\
\text { your sponsor, if applicable. }\end{array}$ & IRB-HSR & $\begin{array}{l}\text { Within } 7 \text { calendar } \\
\text { days from the } \\
\text { time the study } \\
\text { team received } \\
\text { knowledge of the } \\
\text { event. }\end{array}$ & $\begin{array}{l}\text { Protocol Violation, } \\
\text { Noncompliance and } \\
\text { Enrollment Exception } \\
\text { Reporting Form } \\
\text { http://www. virginia.edu/vprgs } \\
\text { /irb/hsr_forms.html } \\
\text { Go to } 3^{\text {rd }} \text { bullet from the } \\
\text { bottom. }\end{array}$ \\
\hline Data Breach & $\begin{array}{l}\text { The UVa } \\
\text { Corporate } \\
\text { Compliance and } \\
\text { Privacy Office } \\
\text { ITC: if breach } \\
\text { involves } \\
\text { electronic data } \\
\text { Police if breach } \\
\text { includes items } \\
\text { that are stolen: } \\
\text { Stolen on UVA } \\
\text { Grounds } \\
\text { OR } \\
\text { Stolen off UVa } \\
\text { Grounds- contact } \\
\text { departice }\end{array}$ & $\begin{array}{l}\text { As soon as } \\
\text { possible and no } \\
\text { later than } 24 \\
\text { hours from the } \\
\text { time the incident } \\
\text { is identified. } \\
\text { As soon as } \\
\text { possible and no } \\
\text { later than } 24 \\
\text { hours from the } \\
\text { time the incident } \\
\text { is identified. } \\
\text { IMMEDIATELY. }\end{array}$ & 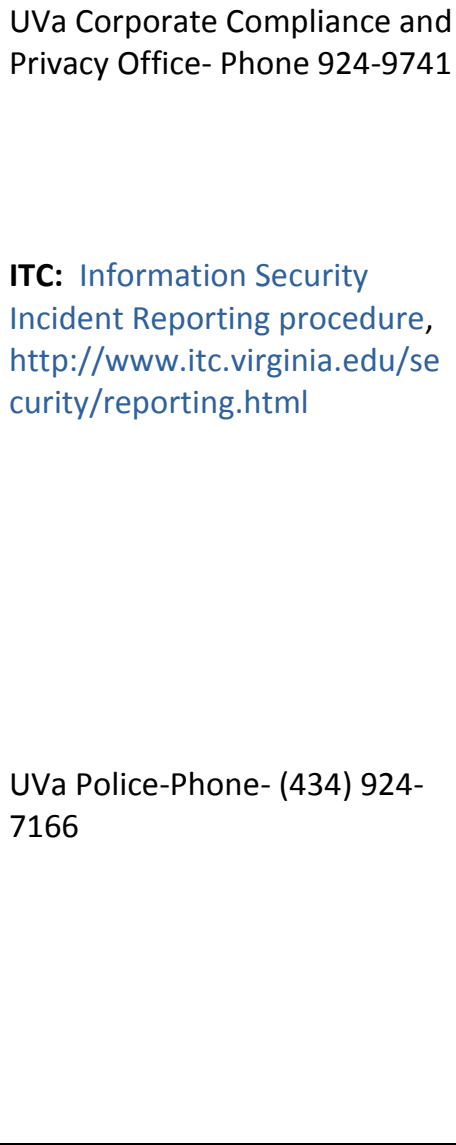 \\
\hline
\end{tabular}

Version Date: 09/06/16

Page Number: 22 of 47 
IRB-HSR \# 19248

Parametric Analysis of Fatigue in Stationary Biking: A Computational Approach

\begin{tabular}{|l|l|l|l|}
\hline & $\begin{array}{l}\text { jurisdiction of last } \\
\text { known location of } \\
\text { PHI }\end{array}$ & & \\
& & & \\
& & & \\
\hline
\end{tabular}

4. How will the endpoint data be collected/recorded. Check all that apply

Protocol specific case report forms

Source documents

X_Database: Specify Answer/Response: Internal UVA Owned Storage Device Other: Specify Answer/Response:

\section{Data and Safety Oversight Responsibility}

\subsection{Who is responsible for overseeing safety data for this study?}

INSTRUCTIONS:

e.g. Who is looking at data in aggregate form to identify trends?

Check all that apply

$\mathrm{X}$ No additional oversight body other than PI at UVa Skip question 5.2

All site PI's (for protocols in which there is no common protocol but data from multiple sites will be combined for analysis: Collaborative Site Analysis Studies)

The UVa Cancer Center Data and Safety Monitoring Committee

If your study involves cancer patients, see Question \# 6 to help you

decide if you should check this option.

Medical Monitor

This could include such things as the overall PI of a multisite trial.

DSMB/ DSMC

If your study is NIH funded, check with the center to determine if they require a DSMB for this study.

The following groups/individuals are NOT considered a DSMB:

DO NOT CHECK THIS BOX

- Members of the study team

- Medical monitors from a commercial drug company

Version Date: 09/06/16

Page Number: 23 of 47 
IRB-HSR \# 19248

Parametric Analysis of Fatigue in Stationary Biking: A Computational Approach

- Safety review committee within a commercial drug company

- UVa Cancer Center Data and Safety Monitoring Committee

- The overall PI of a multi-site trial or a single individual who may serve as the medical monitor.

The following groups are considered a DSMB:

- A group of scientists, physicians, statisticians and others that are not employees of the commercial drug company that are appointed to oversee the data and safety of subjects in the study.

- A group of scientists, physicians, statisticians and others that are, or are not, employees of UVa, are not affiliated with the UVa study team, and are appointed to oversee the data and safety of the subjects in the study.

DSMB Charter Template

A template for a DSMB charter may be found on the IRB-HSR Website at DSMB Charter Template.

Research Monitor: Insert Name

Name required for protocols funded by the Department of Defense

Other: Specify Answer/Response:

5.2. What is the composition of the reviewing body and how is it affiliated with the sponsor?

Members of the study team may NOT also be members of the DMSB.

Information may be found in the UVa Cancer Center Institutional DSMP

Collaborative Site Analysis Study- see CSAS section of this DSMP

Other- Specify Answer/Response:

5.3. What items will be included in the aggregate review conducted by the PI? Check all that apply.

NA- PI is not the overall person overseeing the safety data for this study.

All adverse events

X__Unanticipated Problems

Protocol violations/Issues of noncompliance

Audit results

Application of dose finding escalation/de-escalation rules

Version Date: 09/06/16

Page Number: 24 of 47 
IRB-HSR \# 19248

Parametric Analysis of Fatigue in Stationary Biking: A Computational Approach

These should be outlined under 2.4 .

Application of study designed stopping/decision rules

Early withdrawals

Whether the study accrual pattern warrants continuation/action

Endpoint data

Other: Specify Answer/Response:

\subsection{How often will aggregate review occur?}

For additional information on aggregate review see:

www.virginia.edu/vpr/irb/hsr/continuations.html\#aggreview

NA- PI is not the overall person overseeing the safety data for this study.

Per Enrollment/Events

_._Annually

Semi-Annually

Quarterly

Monthly

Other: Specify Answer/Response:

5.5. How often will a report, regarding the outcome of the review by the DSMB/DSMC, be sent to the UVa PI?

A copy of these reports must be sent to the IRB if applicable as soon as they are received by the PI. Do not wait until the next continuation to submit them to the IRB.

X_NA-PI is the overall person overseeing the safety data for this study.

Per Enrollment/Events

Annually

Semi-Annually

Quarterly

Monthly

Other: Specify Answer/Response:

5.6. How will a report of the information discussed in question 5.4 OR 5.5 be submitted to the IRB? 
IRB-HSR \# 19248

Parametric Analysis of Fatigue in Stationary Biking: A Computational Approach

X Part of IRB-HSR continuation status form

Separate report from DSMB/DSMC or UVa PI

Other: Specify Answer/Response:

Version Date: 09/06/16

Page Number: 26 of 47 


\section{Payment}

1. Are subjects being reimbursed for travel expenses?

Answer/Response: No

IF YES, explain rate/ amount/ upper limits of reimbursements.

Answer/Response: N/A

IF YES, Do you confirm you are aware of the following procedures to follow for reimbursements?

Answer/Response: N/A

2. Are subjects compensated for being in this study?

Answer/Response: Yes

IF YES, answer the following questions (2a-2d).

2a. What is the maximum TOTAL compensation to be given over the duration of the protocol?

Answer/Response: \$50 US

2b. Explain compensation to be given.

Answer/Response: $\$ 50$ US will be given to volunteers following completion of study testing routine via VISA Gift Card.

2c. Is payment pro-rated?

e.g. some compensation is given even if subjects do not complete the entire study Answer/Response: No

If No, explain why payment cannot be pro-rated.

Answer/Response: Short duration of testing makes pro-rating of compensation impractical in the scope of the experiment.

2d. Is money paid from UVa or State funds (including grant funds) or will items such as gift cards be distributed through UVa?

Answer/Response: Yes

IF YES, answer the following questions [2d(i)-2d(ii)].

$2 d(i)$. How will the researcher compensate the subjects?

Check issued to participant via UVA Oracle or State system

Version Date: 09/06/16

Page Number: 27 of 47 
IRB-HSR \# 19248

Parametric Analysis of Fatigue in Stationary Biking: A Computational Approach

Petty cash account*

*Per UVa Policy petty cash payments are limited to a maximum of $\$ 100$ per payment and $\$ 599$ per calendar year per individual.

X Gift card/Debit Card

Other type of compensation:

Specify Answer/Response:

2d(ii). Which category/ categories best describes the process of compensation?

Choose one of the following 3 options

All compensation will be made via check issued to participant via UVA Oracle or State system

The preferred method

Compensation will include an alternative method (petty cash, gift card, other) and tax information will be collected, securely stored, and submitted electronically to Procurement Services as required.

If this box is checked and an alternate method will be used, justify why you are unable to issue checks through the UVa Oracle or state system.

Answer/Response:

IMPORTANT: If you check this box you will be required to submit the subjects' name, Social Security number, full address and amount of payment to Procurement at the end of each calendar year. The Office of the VP for Research will send you instructions on this procedure at a later date.

If the sponsor is providing the gift card/debit card and sending to UVA study team for distribution, please include the statement "SPONSOR REQUEST" under the request for justification.

Version Date: 09/06/16

Page Number: 28 of 47 
IRB-HSR \# 19248

Parametric Analysis of Fatigue in Stationary Biking: A Computational Approach

$X$ Compensation will include an alternative method (petty cash, gift card, other) and tax information cannot be collected. Total possible compensation per participant for participating in the research study over one year is limited to $<=\$ 50$.

- If an alternate method will be used justify why you are unable to issue checks through the UVa Oracle or state system:

Answer/Response: Subjects will be compensated $\$ 50$ or less per year for this protocol and subjects may hesitate to enroll in the study if it requires they share their Social Security number for such a small amount of money.

- If you are unable to collect the tax information justify why it cannot be collected.

Answer/Response: Subjects will be compensated $\$ 50$ or less per year for this protocol and subjects may hesitate to enroll in the study if it requires they share their Social Security number for such a small amount of money.

\section{Risk/ Benefit Analysis}

1. What are the potential benefits for the participant as well as benefits which may accrue to society in general, as a result of this study?

Answer/Response:

- Cardiovascular exercise of Volunteer

- Understanding of relation between kinematics, fatigue, and long-term injury risk

- Foundation for generalizable long-term injury risk model

2. Do the anticipated benefits justify asking subjects to undertake the risks? Answer/Response:

- Yes. While volunteers will undergo typical exercise routines, the resulting fatigue experienced will allow for the collection of muscle activation, kinematic, and kinetic data. Equipped with such data, the investigators in this study will provide a foundation in developing long-term injury models, which can be used to optimize training technique and guide sports device development.

Version Date: 09/06/16

Page Number: 29 of 47 
IRB-HSR \# 19248

Parametric Analysis of Fatigue in Stationary Biking: A Computational Approach

\section{Bibliography}

1. Abbiss, Chris R., Jeremiah J. Peiffer, and Paul B. Laursen. "Optimal cadence selection during cycling." International Sportmed Journal 10.1 (2009): 1-15

2. Bini, Rodrigo R., Fernando Diefenthaeler, and Carlos Bolli Mota. "Fatigue effects on the coordinative pattern during cycling: Kinetics and kinematics evaluation." Journal of Electromyography and Kinesiology 20 (2010): 102-107

3. Bini, Rodrigo R., et al. "Three-dimensional kinematics of competitive and recreational cyclists across different workloads during cycling." European journal of sport science (2016): 1-7.

4. Cote, Julie N., D. Raymond, P. A. Mathieu, A. G. Feldman, and M. F. Levin. "Differences in multi-joint kinematic patterns of repetitive hammering in healthy, fatigued and shoulder-injured individuals.” Clinical Biomechanics 20 (2005): 581-590

5. Gregor, Robert J., Jeffrey P. Broker, And Mary Margaret Ryan. "The Biomechanics of Cycling." Exercise and sport sciences reviews 19.1 (1991): 127-170.

6. Kautz, S. A., and M. L. Hull. "A theoretical basis for interpreting the force applied to the pedal in cycling." Journal of biomechanics 26.2 (1993): 155-165.

7. Kwan, Christy. "Benchmarking progress of bicycling and walking across the US." 143rd APHA Annual Meeting and Exposition (October 31-November 4, 2015). APHA, 2015.

8. Sanderson, David J., Edward M. Hennig, and Alec H. Black. "The influence of cadence and power output on force application and in-shoe pressure distribution during cycling by competitive and recreational cyclists.” Journal of Sports Sciences 18 (2000): 173-181

\section{APPENDIX: Legal/Regulatory}

\section{Recruitment}

The following procedures will be followed:

- Finders fees will not be paid to an individual as they are not allowed by UVa Policy.

- All recruitment materials will be approved by the IRB-HSR prior to use. They will be submitted to the IRB after the IRB-HSR has assigned an IRB-HSR \# to the protocol.

Version Date: 09/06/16

Page Number: 30 of 47 
IRB-HSR \# 19248

Parametric Analysis of Fatigue in Stationary Biking: A Computational Approach

- Only those individuals listed as personnel on this protocol will recruit and or conduct the consenting process with potential subjects.

\section{Retention Incentives}

Any item used by the sponsor/ study team to provide incentive to a subject to remain in the study, other than compensation identified in the Payment section, will be submitted to the IRB for review prior to use. The IRB-HSR will provide the study team with a Receipt Acknowledgement for their records. Retention incentive items are such things as water bottles, small tote bags, birthday cards etc. Cash and gift cards are not allowed as retention incentives.

\section{Clinical Privileges}

The following procedures will be followed:

- Investigators who are members of the clinical staff at the University of Virginia Medical Center must have the appropriate credentials and been granted clinical privileges to perform specific clinical procedures whether those procedures are experimental or standard.

- The IRB cannot grant clinical privileges.

- Performing procedures which are outside the scope of the clinical privileges that have been granted may result in denial of insurance coverage should claims of negligence or malpractice arise.

- Personnel on this protocol will have the appropriate credentials and clinical privileges in place before performing any procedures required by this protocol.

- Contact the Clinical Staff Office- 924-9055 or 924-8778 for further information.

\section{Sharing of Data/Specimens}

Data and specimens collected under an IRB approved protocol are the property of the University of Virginia. You must have "permission" to share data/ specimens outside of UVa other than for a grant application and or publication. This "permission" may come in the form of a contract with the sponsor or a material transfer agreement (MTA) with others. A contract/ MTA is needed to share the data outside of UVa even if the data includes no HIPAA identifiers and no code that could link the data back to a HIPAA identifier.

- No data will be shared outside of UVa, beyond using data for a grant application and or publication, without a signed contract/MTA approved by the SOM Grants and Contracts office/ OSP or written confirmation that one is not needed.

- No specimens will be shared outside of UVa without a signed contract/MTA approved by the SOM Grants and Contracts office/ OSP or written confirmation that one is not needed.

\section{Prisoners}

If the original protocol/ IRB application stated that no prisoners would be enrolled in this study and subsequently a subject becomes a prisoner, the study team must notify the IRB immediately. The study team and IRB will need to determine if the subject will remain in the study. If the subject will remain in the study, the protocol will have to be re-reviewed with the input of a prisoner advocate. The prisoner advocate will also have to be involved in the review

Version Date: 09/06/16

Page Number: 31 of 47 
IRB-HSR \# 19248

Parametric Analysis of Fatigue in Stationary Biking: A Computational Approach

of future continuations, modifications or any other reporting such as protocol violations or adverse events.

Prisoner- Individuals are prisoners if they are in any kind of penal institution, such as a prison, jail, or juvenile offender facility, and their ability to leave the institution is restricted. Prisoners may be convicted felons, or may be untried persons who are detained pending judicial action, for example, arraignment or trial.

For additional information see the OHRP website at

http://www.hhs.gov/ohrp/policy/populations/index.html

\section{Compensation in Case of Injury}

If a subject requests compensation for an injury, the study team should notify the IRB-HSR (9249634/2439847) the UVa Health System Patient Relations Department (924-8315). As a proactive courtesy, the study team may also notify UVa Health System Patient Safety and Risk Management (924-5595).

On request, the study team should provide the Risk Management Office with the following information/documents:

- Subject Name and Medical Record Number

- Research medical records

- Research consent form

- Adverse event report to IRB

- Any letter from IRB to OHRP

\section{Subject Complaints}

During a research study, the study team may receive complaints from a subject. If the study team is uncertain how to respond to a complaint, or is unable to resolve it with the subject, the study team may contact the IRB-HSR (924-9634/243-9847), the UVa Health System Patient Relations Department (924-8315).

\section{Request for Research Records from Search Warrant or Subpoena}

If the study team receives a request for research records from a search warrant or subpoena, they should notify UVa Health Information Services at 924-5136. It is important to notify them if information from the study is protected by a Certificate of Confidentiality.

\section{APPENDIX: Recruitment}

Recruitment includes identifying, review of records to determine eligibility or any contact to determine a potential subjects interest in the study.

*The UVa HIPAA covered entity is composed of the UVa VP Office of Research, the Health System, School of Medicine, School of Nursing, Nutrition Services (Morrisons), the Sheila C. Johnson Center, the Exercise and Sports Injury Laboratory and the Exercise Physiology Laboratory.

Version Date: 09/06/16

Page Number: 32 of 47 
IRB-HSR \# 19248

Parametric Analysis of Fatigue in Stationary Biking: A Computational Approach

\section{How do you plan to identify potential subjects?}

- To "identify" a potential subject refers to steps you plan to take to determine which individuals would qualify to participate in your study. This does NOT include steps to actually contact those individuals.

- If your study involves more than one group of subjects (e.g. controls and cases or subjects and caregivers) note below which groups are being identified by the given method.

- Check the methods you plan to utilize:

a.___ Chart Review/ Clinic Schedule Review/ Database Review from a database established for health care operations (departmental clinical database) or an Improvement Project (e.g. Performance Improvement, Practice Improvement, Quality Improvement).

If you plan to obtain data from the UVa Enterprise Data Warehouse (EDW) please see option b below.

DHHS: Study team requests Waiver of Consent to identify potential subjects.

HIPAA: Allowed under Preparatory to Research if PHI to be accessed.

\section{IMPORTANT}

Keep in mind that $\mathrm{PHI}$ in the medical record may only be accessed by individuals who work under the UVa HIPAA covered entity; which means they meet one of the following criteria:

--a UVa student working in the UVa HIPAA Covered Entity*

--a faculty or staff member in a PAID appointment in the UVA HIPAA

Covered Entity* 
IRB-HSR \# 19248

Parametric Analysis of Fatigue in Stationary Biking: A Computational Approach

b Review of a database that was established to keep data to be used for future research such as the CDR, departmental research database or use of data from a separate current active research protocol.

If you plan to obtain data from the UVa Enterprise Data Warehouse (EDW) you are required to submit your request to the CDR. The CDR staff will work with the EDW to obtain the data you need.

DHHS: Study team requests Waiver of Consent to identify potential subjects.

HIPAA: Allowed under Preparatory to Research if PHI to be accessed.

\section{IMPORTANT}

Keep in mind that PHI in the medical record may only be accessed by individuals who work under the UVa HIPAA covered entity; which means they who meet one of the following criteria:

--a UVa student working in the UVa HIPAA Covered Entity*

--a faculty or staff member in a PAID appointment in the UVA HIPAA Covered Entity*

The information from which you are obtaining potential subjects must also have an IRB protocol approval. If this item is checked, enter the IRB \# below.

IRB\#

If obtaining information from the Clinical Data Repository (CDR) insert IRB \# 10797

c. Patients UVa health care provider supplies the UVa study team with the patients contact information without patients' knowledge.

DHHS: Study team requests Waiver of Consent to identify potential subjects.

HIPAA: Allowed under Preparatory to Research if PHI will be shared by the health care provider.

\section{IMPORTANT}

Keep in mind that PHI may only be given to individuals who work under the UVa HIPAA covered entity; which means they meet one of the following criteria:

--a UVa student working in the UVa HIPAA Covered Entity*

Version Date: 09/06/16

Page Number: 34 of 47 
IRB-HSR \# 19248

Parametric Analysis of Fatigue in Stationary Biking: A Computational Approach

--a faculty or staff member in a PAID appointment in the UVA HIPAA Covered Entity*

d. Patient obtains information about the study from their health care provider. The patient contacts the study team if interested in participating. (Health care provider may or may not also be the a member of the study team)

DHHS: NA

HIPAA: Allowed under Health Care Operations

If this choice is checked, check 3d-INDIRECT CONTACT below.

e. $\quad \underline{X}$ Potential subjects will not be directly identified. They will respond to an advertisement such as a flyer, brochure etc. Email will be sent to biking clubs in region to inform potential subjects of the study.

If this choice is checked, check 3d- INDIRECT CONTACT below.

DHHS \& HIPAA: NA

f. Potential subjects have previously signed a consent to have their name in a registry/database to be contacted for future studies of this type.

IRB\# of registry/ database:

DHHS \& HIPAA: NA

g. ___ Other: Specify Answer/Response:

If item \# $\mathrm{a}, \mathrm{b}$ or $\mathrm{c}$ is checked above and if this protocol involves the use of protected health information do you confirm the following to be true?

- The use or disclosure is sought solely to review protected health information as necessary to prepare the research protocol or other similar preparatory purposes.

- No PHI will be removed from the UVa covered entity.

- The PHI that the researcher seeks to use or access is necessary for the research purposes.

Answer/Response:

Version Date: 09/06/16

Page Number: 35 of 47 
IRB-HSR \# 19248

Parametric Analysis of Fatigue in Stationary Biking: A Computational Approach

\section{How will potential subjects be contacted?}

To "contact" a potential subjects refers to the initial contact you plan to take to reach a potential subject to determine if they would be interested in participating in your study. This may include direct contact by such methods as by letter, phone, email or in-person or indirect contact such as the use of flyers, radio ads etc.

If your study involves more than one group of subjects (e.g. controls and cases or subjects and caregivers) note below which groups are being contacted by the given method.

Check the methods below you plan to utilize:

a.___ Direct contact of potential subjects by the study team via letter, phone, direct e-mail. Members of study team ARE NOT health care providers of patients. Information will not be collected from psychotherapy notes.

Note: Letter, phone, direct email scripts must be approved by IRB prior to use. See IRB-HSR Website for templates.

DHHS/HIPAA: Study team requests a Waiver of Consent and Waiver of HIPAA Authorization to contact potential subjects.

\section{IMPORTANT:}

Keep in mind that if PHI was collected during the identification phase that contact with potential subjects may only be performed by individuals who work under the UVa HIPAA covered entity; which means they meet one of the following criteria:

- a UVa student working in the UVa HIPAA Covered Entity*

- a faculty or staff member in a PAID appointment in the UVA HIPAA Covered Entity*

b.___ Potential subjects will be approached while at UVa Hospital or Health Clinic by a person who is NOT a member of their health care team. Information will not be collected from psychotherapy notes.

DHHS \& HIPAA: Study team requests a Waiver of Consent and a Waiver of HIPAA Authorization to contact potential subjects.

\section{IMPORTANT:}

Keep in mind that contacting individuals in a clinical setting may only be performed by individuals who work under the UVa HIPAA covered entity; which means they meet one of the following criteria:

a UVa student working in the UVa HIPAA Covered Entity*

Version Date: 09/06/16

Page Number: 36 of 47 
IRB-HSR \# 19248

Parametric Analysis of Fatigue in Stationary Biking: A Computational Approach

a faculty or staff member in a PAID appointment in the UVA HIPAA Covered Entity*

You should share the following information with the potential subject:

- Your name

- Who you are: physician, nurse etc. at the University of Virginia.

- Why you want to speak with them

- Ask if you have their permission to explain the study to them

- If asked about how you obtained their information use one of the following as an option for response.

- DO NOT USE THIS RESPONSE UNLESS YOU HAVE OBTAINED PERMISSION FROM THEIR UVa PHYSICIAN: Your doctor, Dr. insert name wanted you to be aware of this research study and gave us permission to contact you.

- We obtained your information from your medical records at UVa.

- Federal regulations allow the UVa Health System to release your information to researchers at UVa, so that we may contact you regarding studies you may be interested in participating. We want to assure you that we will keep your information confidential.

- IF THE PERSON SEEMS ANGRY, HESITANT OR UPSET, THANK THEM FOR THEIR TIME AND DO NOT ENROLL THEM IN THE STUDY. YOU MAY ALSO REFER THEM TO THE IRB-HSR AT 924-9634.

c.___ Direct contact of potential subjects by the study team by approaching in person at UVa or via letter, phone, direct e-mail. Members of study team contacting potential subjects ARE health care providers of patients.

If you are not approaching them in person but using a letter, phone call or direct email please note that the letter, phone, direct email scripts must be approved by IRB prior to use.

See IRB-HSR Website for templates.

DHHS: Study team requests a Waiver of Consent to contact potential subjects

HIPAA: Allowed under Health Care Operations.

d. X Indirect contact (flyer, brochure, TV, broadcast emails, patient provided info about the study from their health care provider and either the patient contacts study team or gives their healthcare provider permission for the study team to contact them.)

Version Date: 09/06/16

Page Number: 37 of 47 
IRB-HSR \# 19248

Parametric Analysis of Fatigue in Stationary Biking: A Computational Approach

The indirect method used (flyer, brochure, TV, broadcast emails) must be approved by the IRB prior to use. The IRB does not need to review any type of script to use when the potential subject responds to the indirect method.

DHHS \& HIPAA: NA

e.

Potential subjects are not patients. The study does not include obtaining subjects health information. Subjects will be contacted directly via email, phone, letter or presentation in group setting with consent then obtained individually in a private setting.

If you are not approaching them in person but using a letter, phone call or direct email please note that the letter, phone, direct email scripts must be approved by IRB prior to use.

See IRB-HSR Website for templates.

DHHS: Study team requests a Waiver of Consent to contact potential subjects.

HIPPA: NA

\section{Will any additional information be obtained from a potential subject during} "prescreening"?

Pre-screening for IRB purposes is the term used to describe activities PRIOR to obtaining Informed Consent and may not include any research procedures.

The activities may involve pre-screening of potential subjects over the telephone or in person is generally performed to determine their initial eligibility for, and, interest in a study and is a common strategy in the recruitment process.

Questions appropriate for pre-screening address the specific inclusion/exclusion criteria for the study and other issues of suitability, for example, an individual's ability to come to the research site multiple times.

It is not appropriate at this point in the process (i.e. prior to obtaining informed consent/enrollment) to gather information that is not directly related to assessing eligibility and suitability (e.g. obtaining complete medical histories, obtaining blood specimens for lab tests).

An additional telephone script is not required, for this pre-screening process, in addition to any scripts required under Recruitment question \# 2.

Answer/Response: Only questions pertaining to inclusion/exclusion will be asked.

IF YES, submit any documents that will be used to collect pre-screening information so that the IRB may confirm what questions will be asked.

Version Date: 09/06/16

Page Number: 38 of 47 
Parametric Analysis of Fatigue in Stationary Biking: A Computational Approach

NOTE: To comply with HIPAA regulations only the minimum necessary information may be collected at this time. This means that only questions pertaining to the Inclusion and Exclusion Criteria may be asked.

\section{IF YES,}

DHHS: study team requests a Waiver of Documentation of Consent for Prescreening questions.

HIPPA:

HIPAA does not apply if:

--no PHI is collected or

--if $\mathrm{PHI}$ is collected from a potential subject by an individual from a department that is not part of the HIPAA covered entity.

HIPAA does apply if the collection occurs by individuals* who work in a department that is part of the HIPAA covered entity.

In this case the collection will be covered under Health Care Operations/

These individuals are those that meet one of the following criteria:

--a UVa student working in the UVa HIPAA Covered Entity*

--a faculty or staff member in a PAID appointment in the UVA HIPAA Covered Entity*

IF YES, Will any of the questions involve health information?

Answer/Response: Yes

IF YES, will you collect HIPAA identifiers with the health information?

Answer/Response: Yes

IF YES, which HIPAA identifiers will be recorded?

Answer/Response: Name and Age (To Confirm Identification)

Do you confirm that health information with HIPAA identifiers will not be shared outside of UVa until a consent form is signed or only shared in a de-identified manner?

Answer/Response: Yes 
IRB-HSR \# 19248

Parametric Analysis of Fatigue in Stationary Biking: A Computational Approach

4. Do you plan to ask the subjects to do anything, other than answering questions, for the study prior to signing a consent?

For example: come to the first visit fasting, stop taking medications that may be an exclusion criteria, change diet. As this is still part of pre-screening one is not allowed to gather information that is not directly related to inclusion/exclusion criteria or other issues of suitability (e.g. is person able to come to UVa for multiple visits)

NOTE:

Only those members of the study team with a DEA\# (license to prescribe drugs) are allowed to determine if a potential subject may be asked/informed to stop taking a drug which is an exclusion criteria.

It is recommended that the potential subject notify their health care provider if they plan to stop a prescription drug.

Answer/Response: No

IF YES, explain in detail what you will ask them to do. Answer/Response:

\section{Tips to Study Team}

You must document their verbal consent in the study records.

If a subject is asked to stop taking a drug, document the date and name of the person on the study team giving the verbal order to stop medications (againmust be a person with a DEA\#).

DHHS: Study team requests the use of Verbal Consent (Waiver of Documentation of Consent) for minimal risk screening procedures.

HIPPA:

If the individual, obtaining consent, works under the HIPAA Covered Entity this is covered under Health Care Operations

If the individual obtaining consent does not work under the HIPAA covered entity, HIPAA does not apply.

5. How will the consenting process take place with either the prospective subject, the subject's legally authorized representative or parent/legal guardian of a minor (if applicable)?

HIPPA:

If the individual, obtaining consent, works under the HIPAA Covered Entity consenting is covered under Health Care Operations.

If the individual obtaining consent does not work under the HIPAA covered entity, HIPAA does not apply. 
IRB-HSR \# 19248

Parametric Analysis of Fatigue in Stationary Biking: A Computational Approach

Describe the setting for the consent.

Who will discuss the study with the potential subject?

Where will the consenting process take place?

How will you assess subject understanding?

How much time will pass between obtaining written consent and initiation of study procedures?

See Protocol Examples: Consenting Process for examples of how to answer this question.

If recruiting minors, specify how parental /guardian consent will be obtained prior to approaching the minor.

Answer/Response:

- Discussion with Subject: Sub-Investigator \& Study Coordinator I

- Consenting Location:

Pilkey Conference Room

Center for Applied Biomechanics

University of Virginia

4040 Lewis and Clark Drive

Charlottesville, Virginia 22911

- Understanding: Presentation and Thorough Q\&A session following Briefing (See "Consent Overview" Document)

- Elapsed Time After Consent: Immediate

\section{Will subjects sign a consent form for any part of the study?}

Answer/Response: Yes

7. Will the study procedures be started the same day the subject is recruited for the study?

Answer/Response: Yes

IF YES, explain in detail why the subject cannot be given more time to make a decision to consent.

Answer/Response: Due to the necessary commute to Center for Applied Biomechanics, potential consenting volunteers will only make one visit to the center, during which written consent and testing will be conducted.

IF YES, explain in detail what will be done to assure the potential subject has enough time to make an informed decision.

Answer/Response: Upon receiving interest email from potential volunteer, full consent and study information documentation will be provided to the volunteer to review. A pre-screening call will be made with each volunteer to confirm identity, review study details, and gauge the volunteers' "intent to consent". Only after this pre-screen will volunteers schedule a testing session where they are asked to provide written consent and conduct the study's tests.

Version Date: 09/06/16

Page Number: 41 of 47 
8. Is there the potential to recruit economically or educationally disadvantaged subjects, or other vulnerable subjects such as students or employees?

INSTRUCTIONS: If you will be recruiting patients from the UVa Health System, you must answer this question YES as the UVa Health System cares for patients who are economically disadvantaged.

Answer/Response: Yes

IF YES, what protections are in place to protect the rights and welfare of these subjects so that any possible coercion or undue influence is eliminated? Answer/Response:

Students of UVA may be selected as subjects in this study. To reduce the likelihood of undue influence, compensation will be kept moderately low for the provided time, and primarily interested experienced bike riders will be selected from the biking community.

9. Do you need to perform a "dry run" of any procedure outlined in this protocol? A "dry run" is a procedure done to validate the system used to obtain results. It requires a human "subject" however the results of the dry run are used for system validation and not for the actual research. A common example a "dry run" is the validation or qualification MRI scans required by sponsor to ensure the MRI at UVa is able to perform the study-required scans.

- If you are doing a sponsored study that involves an MRI for research, you are encouraged to say YES to this question

- If YES, complete and submit a Consent for a Dry Run Procedure

- A template for a Consent for Dry-Run MRI is located under FORMS on the IRB Website

- IF YES, answer the following questions.

Answer/Response: No 
IRB-HSR \# 19248

Parametric Analysis of Fatigue in Stationary Biking: A Computational Approach

\section{Privacy Plan}

The following procedures must be followed.

- The data will be secured per the Data Security Plan of this protocol.

- Only investigators for this study and clinicians caring for the patient will have access to data. They will each use a unique login ID and password that will keep confidential. The password should meet or exceed the standards described on the Information Technology Services (ITS) webpage about The Importance of Choosing Strong Passwords.

- Each investigator will sign the University's Electronic Access Agreement forward the signed agreement to the appropriate department as instructed on the form.

If you currently have access to clinical data it is likely that you have already signed this form. You are not required to sign it again.

- UVa University Data Protection Standards will be followed http://www.virginia.edu/informationsecurity/dataprotection.

- If identifiable data is transferred to any other location such as a desktop, laptop, memory stick, CD etc. the researcher must follow the University's "Electronic Storage of Highly Sensitive Data Policy". Additional requirements may be found in the University's Requirements for Securing Electronic Devices.

- If identifiable data is taken away from the UVa Health System, Medical Center Policy \# 0218 will be followed.

- Data will be securely removed from the server/drive, additional computer(s), and electronic media according to the University's Electronic Data Removal Policy.

- Data will be encrypted or removed if the electronic device is sent outside of UVa for repair according to the University's Electronic Data Removal Policy.

- If PHI will be faxed, researchers will follow the Health System Policy \# 0194.

- If PHI will be emailed, researchers will follow the Health System Policy \# 0193 and University Data Protection Standards.

- Data may not be analyzed for any other study without additional IRB approval.

- If you are using patient information you must follow Health System Policy \# 0021.

- Both data on paper and stored electronically will follow the University's Record Management policy and the Commonwealth statute regarding the Destruction of Public Records.

Summary of Requirements to Comply with UVa Health System, Medical Center and University Policies and Guidance as noted above:

\section{Highly Sensitive Data is:}

-personal information that can lead to identity theft if exposed or -data that reveals an individual's health condition and/or history of health services use.

Protected Data (PHI) a type of Highly Sensitive Data, is data combined with a HIPAA identifier Identifiable Data under HIPAA regulations is considered to be Highly Sensitive Data at UVa. A Limited Data Set (LDS) under HIPAA regulations is considered to be Moderately Sensitive Data at UVa. The only HIPAA identifiers associated with data: dates and or postal address information limited to town or city, state, and zip code.

Will not include subjects age if older than 89 or subjects DOB if older than 89.

Version Date: 09/06/16

Page Number: 43 of 47 
IRB-HSR \# 19248

Parametric Analysis of Fatigue in Stationary Biking: A Computational Approach

\begin{tabular}{|c|c|}
\hline $\begin{array}{l}\text { Highly Sensitive Data } \\
\text { (Identifiable Health Info per HIPAA) }\end{array}$ & $\begin{array}{l}\text { Moderately Sensitive Data } \\
\text { (Limited Data Set and De-identified data per } \\
\text { HIPAA) }\end{array}$ \\
\hline General Issues & General Issues \\
\hline $\begin{array}{l}\text { Discussions in private } \\
\text { Do not share with those not on the study team or } \\
\text { those who do not have a need to know. }\end{array}$ & $\begin{array}{l}\text { Do not share with those not on the study team or } \\
\text { those who do not have a need to know }\end{array}$ \\
\hline Password protect & Password protect \\
\hline $\begin{array}{l}\text { Physically secure (lock) hard copies at all times if not } \\
\text { directly supervised. } \\
\text { If not supervised hard copies must have double } \\
\text { protection (e.g. lock on room OR cabinet AND in } \\
\text { building requiring swipe card for entrance). }\end{array}$ & $\begin{array}{l}\text { Physically secure (lock) hard copies at all times if } \\
\text { not directly supervised. }\end{array}$ \\
\hline $\begin{array}{l}\text { For electronic documents turn off File Sharing; turn } \\
\text { on firewalls; use up to date antivirus and } \\
\text { antispyware; delete data securely. }\end{array}$ & $\begin{array}{l}\text { For electronic documents turn off File Sharing; turn } \\
\text { on firewalls; use up to date antivirus and } \\
\text { antispyware; delete data securely. }\end{array}$ \\
\hline $\begin{array}{l}\text { Encrypt } \\
\text { See Encryption Solutions Guidance } \\
\text { Files on Health System Network drives are } \\
\text { automatically encrypted. If not stored there it is study } \\
\text { teams responsibility to make sure data are encrypted. }\end{array}$ & \\
\hline $\begin{array}{l}\text { If device sent out for service or repair, encrypt or } \\
\text { remove data AND contract for repair using a UVa } \\
\text { Purchase order. }\end{array}$ & $\begin{array}{l}\text { If device sent out for service or repair, encrypt or } \\
\text { remove data AND contract for repair using a UVa } \\
\text { Purchase order. }\end{array}$ \\
\hline $\begin{array}{l}\text { Store files on a network drive specifically designated } \\
\text { for storing this type of data, e.g. high-level security } \\
\text { server/drives managed by Information Technology } \\
\text { Services or the "F" and "O" managed by Heath } \\
\text { Systems Computing Services. You may access it via a } \\
\text { shortcut icon on your desktop, but you are not } \\
\text { allowed to take it off line to a local drive such as the } \\
\text { desktop of your computer (e.g. C drive) or to an } \\
\text { individual Use Device*. May access via VPN }\end{array}$ & \\
\hline $\begin{array}{l}\text { Do not share with sponsor or other outside group } \\
\text { before consent is obtained or the IRB has granted } \\
\text { appropriate approvals and contract/ MTA is in place }\end{array}$ & $\begin{array}{l}\text { Do not share with sponsor or other outside group } \\
\text { before consent is obtained or the IRB has granted } \\
\text { appropriate approvals and contract/ MTA is in } \\
\text { place }\end{array}$ \\
\hline $\begin{array}{l}\text { If collected without consent/ HIPAA authorization } \\
\text { will NOT be allowed to leave UVa HIPAA covered } \\
\text { entity unless disclosure is approved by the IRB and } \\
\text { the disclosure is tracked in EPIC }\end{array}$ & $\begin{array}{l}\text { If collected without consent/ HIPAA authorization } \\
\text { will NOT be allowed to leave UVa HIPAA covered } \\
\text { entity unless disclosure is approved by the IRB and } \\
\text { an MTA is in place prior to sharing of data }\end{array}$ \\
\hline
\end{tabular}

Version Date: 09/06/16

Page Number: 44 of 47 
IRB-HSR \# 19248

Parametric Analysis of Fatigue in Stationary Biking: A Computational Approach

\begin{tabular}{|c|c|}
\hline $\begin{array}{l}\text { Highly Sensitive Data } \\
\text { (Identifiable Health Info per HIPAA ) }\end{array}$ & $\begin{array}{l}\text { Moderately Sensitive Data } \\
\text { (Limited Data Set and De-identified data per HIPAA) }\end{array}$ \\
\hline Electronic Data Collection \& Sharing & Electronic Data Collection \& Sharing \\
\hline $\begin{array}{l}\text { (e.g. smart phone app, electronic consent using } \\
\text { tablet etc.) } \\
\text { MUST consult with ISPRO or Health System Web } \\
\text { Development Office: } 434-243-6702 \\
\text { - University Side: IT- } \\
\text { Security@virginia.edu } \\
\text { - Health System: Web Development Center: }\end{array}$ & \\
\hline Individual-Use Device & Individual-Use Device \\
\hline $\begin{array}{l}\text { Do not save to individual-use device* without } \\
\text { written approval of your Department AND VP } \\
\text { or Dean. } \\
\text { If approval obtained, data must be password } \\
\text { protected and encrypted. }\end{array}$ & \\
\hline $\begin{array}{l}\text { Do not save an email attachment containing } \\
\text { HSD to an individual use device } \\
\text { (e.g. smart phone) }\end{array}$ & \\
\hline E Mail & E Mail \\
\hline $\begin{array}{l}\text { Do not share via email with Outlook Web/ or } \\
\text { forward email using other email vendors like } \\
\text { Gmail/ Yahoo }\end{array}$ & \\
\hline $\begin{array}{l}\text { Do not send via email on smart phone unless } \\
\text { phone is set up by Health System }\end{array}$ & \\
\hline $\begin{array}{l}\text { Email may include name, medical record } \\
\text { number or Social Security number only if } \\
\text { sending email to or from a person with * HS in } \\
\text { their email address. } \\
\text { NOTE: VPR \& IRB staff do not meet this criteria! }\end{array}$ & $\begin{array}{l}\text { In addition to sharing LDS, may include initials if } \\
\text { persons sending and receiving email work within the } \\
\text { UVa HIPAA covered entity. }{ }^{* *}\end{array}$ \\
\hline$F A X$ & $F A X$ \\
\hline Verify FAX number before faxing & Verify FAX number before faxing \\
\hline $\begin{array}{l}\text { Use Fax Cover Sheet with Confidentiality } \\
\text { Statement }\end{array}$ & Use Fax Cover Sheet with Confidentiality Statement \\
\hline $\begin{array}{l}\text { Verify receiving fax machine is in a restricted } \\
\text { access area }\end{array}$ & $\begin{array}{l}\text { Verify receiving fax machine is in a restricted access } \\
\text { area }\end{array}$ \\
\hline Verify intended recipient is clearly indicated & Verify intended recipient is clearly indicated \\
\hline $\begin{array}{l}\text { Recipient is alerted to the pending transmission } \\
\text { and is available to pick it up immediately }\end{array}$ & $\begin{array}{l}\text { Recipient is alerted to the pending transmission and } \\
\text { is available to pick it up immediately }\end{array}$ \\
\hline
\end{tabular}

Version Date: 09/06/16

Page Number: 45 of 47 
IRB-HSR \# 19248

Parametric Analysis of Fatigue in Stationary Biking: A Computational Approach

\begin{tabular}{|c|c|}
\hline $\begin{array}{l}\text { Highly Sensitive Data } \\
\text { (Identifiable Health Info per HIPAA) }\end{array}$ & $\begin{array}{l}\text { Moderately Sensitive Data } \\
\text { (Limited Data Set and De-identified data per HIPAA) }\end{array}$ \\
\hline Electronic Data Collection \& Sharing & Electronic Data Collection \& Sharing \\
\hline $\begin{array}{l}\text { (e.g. smart phone app, electronic consent } \\
\text { using tablet etc.) } \\
\text { MUST consult with ISPRO or Health System } \\
\text { Web Development Office: 434-243-6702 } \\
\text { - University Side: IT- } \\
\quad \text { Security@virginia.edu } \\
\text { - Health System: Web Development Center: }\end{array}$ & \\
\hline Individual-Use Device & Individual-Use Device \\
\hline $\begin{array}{l}\text { Do not save to individual-use device* without } \\
\text { written approval of your Department AND VP } \\
\text { or Dean. } \\
\text { If approval obtained, data must be password } \\
\text { protected and encrypted. }\end{array}$ & \\
\hline $\begin{array}{l}\text { Do not save an email attachment containing } \\
\text { HSD to an individual use device } \\
\text { (e.g. smart phone) }\end{array}$ & \\
\hline E Mail & E Mail \\
\hline $\begin{array}{l}\text { Do not share via email with Outlook Web/ or } \\
\text { forward email using other email vendors like } \\
\text { Gmail/ Yahoo }\end{array}$ & \\
\hline $\begin{array}{l}\text { Do not send via email on smart phone unless } \\
\text { phone is set up by Health System }\end{array}$ & \\
\hline $\begin{array}{l}\text { Email may include name, medical record } \\
\text { number or Social Security number only if } \\
\text { sending email to or from a person with * HS in } \\
\text { their email address. } \\
\text { NOTE: VPR \& IRB staff do not meet this } \\
\text { criteria! }\end{array}$ & $\begin{array}{l}\text { In addition to sharing LDS, may include initials if } \\
\text { persons sending and receiving email work within the } \\
\text { UVa HIPAA covered entity. }{ }^{* *}\end{array}$ \\
\hline$F A X$ & $F A X$ \\
\hline Verify FAX number before faxing & Verify FAX number before faxing \\
\hline $\begin{array}{l}\text { Use Fax Cover Sheet with Confidentiality } \\
\text { Statement }\end{array}$ & Use Fax Cover Sheet with Confidentiality Statement \\
\hline $\begin{array}{l}\text { Verify receiving fax machine is in a restricted } \\
\text { access area }\end{array}$ & $\begin{array}{l}\text { Verify receiving fax machine is in a restricted access } \\
\text { area }\end{array}$ \\
\hline Verify intended recipient is clearly indicated & Verify intended recipient is clearly indicated \\
\hline $\begin{array}{l}\text { Recipient is alerted to the pending } \\
\text { transmission and is available to pick it up }\end{array}$ & $\begin{array}{l}\text { Recipient is alerted to the pending transmission and } \\
\text { is available to pick it up immediately }\end{array}$ \\
\hline
\end{tabular}

Version Date: 09/06/16

Page Number: 46 of 47 
IRB-HSR \# 19248

Parametric Analysis of Fatigue in Stationary Biking: A Computational Approach

\begin{tabular}{|c|c|}
\hline $\begin{array}{l}\text { Highly Sensitive Data } \\
\text { (Identifiable Health Info per HIPAA ) }\end{array}$ & $\begin{array}{l}\text { Moderately Sensitive Data } \\
\text { (Limited Data Set and De-identified data per HIPAA) }\end{array}$ \\
\hline Electronic Data Collection \& Sharing & Electronic Data Collection \& Sharing \\
\hline $\begin{array}{l}\text { (e.g. smart phone app, electronic consent } \\
\text { using tablet etc.) } \\
\text { MUST consult with ISPRO or Health System } \\
\text { Web Development Office: } 434-243-6702 \\
\text { University Side: IT-Security@virginia.edu } \\
\text { Health System: Web Development Center: } \\
\text { Contract must include required security } \\
\text { measures. }\end{array}$ & \\
\hline $\begin{array}{l}\text { May NOT be stored in places like UVaBox, } \\
\text { UVaCollab, QuestionPro. } \\
\text { May also NOT be stored in non-UVa licensed } \\
\text { cloud providers, such as Dropbox, Google } \\
\text { Drive, SkyDrive, Survey Monkey, etc. }\end{array}$ & $\begin{array}{l}\text { May be stored in places like UVaBox, UVaCollab, } \\
\text { QuestionPro. } \\
\text { May NOT be stored in non-UVa licensed cloud } \\
\text { providers, such as Dropbox, Google Drive, SkyDrive, } \\
\text { Survey Monkey, etc. }\end{array}$ \\
\hline LOST OR STOLEN: & LOST OR STOLEN: \\
\hline $\begin{array}{l}\text { Must report in accordance with protocol/ in } \\
\text { accordance with the Information Security } \\
\text { Incident Reporting Policy. }\end{array}$ & $\begin{array}{l}\text { Must report in accordance with protocol/ in } \\
\text { accordance with the Information Security Incident } \\
\text { Reporting Policy. }\end{array}$ \\
\hline $\begin{array}{l}\text { Any data breach will also be reported to the } \\
\text { IRB of Record if the report meets the criteria } \\
\text { of an Unanticipated Problem. }\end{array}$ & $\begin{array}{l}\text { Any data breach will also be reported to the IRB of } \\
\text { Record if the report meets the criteria of an } \\
\text { Unanticipated Problem. }\end{array}$ \\
\hline
\end{tabular}

* Individual Use Device - examples include smart phone, $C D$, flash (thumb) drive, laptop, C drive of your computer,

**The UVa HIPAA covered entity is composed of the UVa VP Office of Research, the Health System, School of Medicine, School of Nursing, Nutrition Services (Morrison's), the Sheila C. Johnson Center, the Exercise and Sports Injury Laboratory and the Exercise Physiology Laboratory.

Version Date: 09/06/16

Page Number: 47 of 47 


\section{DATA COLLECTION}

\section{A. Will any HIPAA identifiers be collected or received by the UVa study team?}

\begin{tabular}{|c|c|c|}
\hline \multicolumn{3}{|c|}{ INSTRUCTIONS: } \\
\hline \multicolumn{3}{|c|}{$\begin{array}{l}\text { - Answer YES if you are collecting, recording or receiving any of these items for a potential } \\
\text { - Anbject, an enrolled subject, a subject's relative, household member or employer. } \\
\text { collected. } \\
\text { - Keep in mind that the information below includes data collected via photographs, video, } \\
\text { audiotapes, and systems like IVRS (Interactive Voice Response System) } \\
\text { - If you answer NO to all items it means you would never be able to go back and obtain any } \\
\text { additional information about an individual. } \\
\text { - Glossary of terms located at end of document. }\end{array}$} \\
\hline YES & NO & HIPAA Identifier \\
\hline Х & & 1. Name \\
\hline$\bigotimes$ & $\mid 7$ & 2. Postal address information, other than town or city, state, and zip code \\
\hline & $\bigotimes$ & 3. Age if over the age of 89 OR Date of Birth if over the age of 89 \\
\hline$\bigotimes$ & $\square$ & 4. Telephone numbers \\
\hline & 內 & 5. Fax numbers \\
\hline$\bigotimes$ & $\square$ & 6. Electronic mail addresses \\
\hline & $\bar{\varnothing}$ & 7. Social Security number-Must be checked if you are collecting SS\# for compensation. \\
\hline & $\bigotimes$ & 8. Medical Record number \\
\hline & $\bigotimes$ & 9. Health plan beneficiary numbers \\
\hline & $\bar{\bigotimes}$ & 10. Account numbers (e.g. bank numbers, credit card numbers, hospital bill account number) \\
\hline & Х & 11. Certificate/license numbers (e.g. passport number, driver's license number, medical board license number) \\
\hline & $\bigotimes$ & 12. Vehicle identifiers and serial numbers, including license plate numbers \\
\hline & 冈 & 13. Device identifiers and serial numbers \\
\hline & 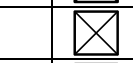 & 14. Web Universal Resource Locators (URLs) \\
\hline & Х & 15. Internet Protocol (IP) address numbers \\
\hline & $\bigotimes$ & 16. Biometric identifiers, including finger and voice prints \\
\hline$\bigotimes$ & $\square$ & 17. Full face photographic images and any comparable images \\
\hline$\square$ & $\bigotimes$ & $\begin{array}{l}\text { 18. Any other unique identifying number, characteristic, code that is derived from or related to information about } \\
\text { the individual (e.g. initials, last } 4 \text { digits of Social Security \#, mother's maiden name, first } 3 \text { letters of last name.) } \\
\text { DO NOT CHECK THIS BOX IF THE SUBJECT ID WILL BE SOMETHING LIKE SUBJECT \#1. }\end{array}$ \\
\hline & $\begin{array}{l}\text { NSTRUC } \\
\text { f you ch } \\
\text { SENSITIV } \\
\text { Eollow re } \\
\text { Do not a }\end{array}$ & $\begin{array}{l}\text { ONS: } \\
\text { red NO to all HIPAA Identifiers above your data is considered to be MODERATELY } \\
\text { airements for handling moderately sensitive data in the Privacy Plan of the protocol. } \\
\text { Ner any additional questions. No review by ISPRO is required. }\end{array}$ \\
\hline
\end{tabular}

Version Date: 8/26/2016

Page 1 of 23

IRB Submission \#10883 
1A(1) Check ALL locations below where DATA WITH HIPAA IDENTIFIERS from Table 1A will be COLLECTED or KEPT together in the same location

(e.g. on the same electronic drive or in the same paper file)

$\bigotimes$ in paper file - If checked LIST all HIPAA identifiers to be stored in paper file:

Name (1), Postal Address Information (2), Telephone Numbers (4), Electronic Email Addresses (6),

Remember: Initials are considered a HIPAA identifier!

If health information with HIPAA identifiers are stored in a paper file, where will the paper files be housed?

$\bigotimes$ Signed consent forms or documentation regarding obtaining verbal consent will be stored in a secure area with limited access.

$\square$ Case report forms will be stored in a secure area with limited access.

$\bigotimes$ Questionnaires/surveys will be stored in a secure area with limited access.

$\square$ Other-Specify

NOTE: "in a secure area with limited access" means access to data is limited to study personnel only and there must be two forms of security. Example: 1) in a locked office in a building with swipe locks when unattended or 2) in a locked file cabinet in a locked room when unattended or 3 ) study personnel present in room at all times located in a building with swipe locks or a room with a lock,

$\bigotimes$ in an electronic file. If checked LIST all HIPAA identifiers in an electronic file:

Comparable Images (17)

Remember: Initials are considered a HIPAA identifier!

1B. Check ALL applicable items below to describe HOW DATA will be COLLECTED:

IMPORTANT: If you check any of the items 1B(1) through 1B(3) below submit ISPRO approval with new protocol submission.

You should consult with ISPRO during the development phase of this protocol if your protocol will involve highly technical issues such as the creation of a website to collect data, software application development, the use of a smart phone app, or if you plan to store identifiable data ONTO a tablet/laptop.

Otherwise submit the protocol and this Data Security Plan to ISPRO for review after the protocol is written. ISPRO does NOT need the Protocol Cover Sheet, Consent Forms or any other ancillary documents. ISPRO CONTACT INFORMATION:

UVa Office of Information Security, Policy \& Records Office (ISPRO)

www.virginia.edu/ispro

Email: IT-Security@Virginia.edu

Version Date: 8/26/2016

Page 2 of 23

IRB Submission \#10883 
1B(1). Collection of data ONTO* an individual-use device (examples include desktop computer, smart phone app, flash (thumb) drive, external hard drive, tablet, laptop, CD, C drive of your computer, camera, video or audio recorder)

- *ONTO means the data will reside on the device. Do not check this box if the device will simply be used to access a server.

If you checked YES to any item in 1A have you previously received ISPRO approval for collecting data ONTO the same individual-use device? Yes $\square$ No $\square$

If NO, COMPLETE DATA SECURITY PLAN: APPENDIX 1B(1).

If YES, submit ISPRO approval with this protocol submission.

1B(2.) $\square$ Collection of data via web-based format (e.g. online consent, online surveys) DO NOT check if data will be collected directly to a server/drive managed by the sponsor or CRO in which data will be sent and stored in an encrypted fashion (e.g. must be shared and stored via Secure FX, Secure FTP, HTTPS, PGP) and the server/drive is configured to store data regulated by HIPAA.

If you checked YES to any item in 1A have you previously received ISPRO approval for collecting data ONTO the same web based format? Yes No $\square$ If NO COMPLETE DATA SECURITY PLAN: APPENDIX 1B(2). If YES, submit ISPRO approval with this protocol submission.

1B(3). $\square$ Directly to a server NOT listed under 1B(4) below.

If you checked YES to any item in 1A have you previously received ISPRO approval for collecting data ONTO the same server/drive/drive managed by ITS? Yes No

If YES, submit ISPRO approval with this protocol submission.

If NO, COMPLETE DATA SECURITY PLAN APPENDIX 1B(3). 
1B(4). $\square$ Directly to one of the servers listed below.

IF CHECKED COMPLETE DATA SECURITY PLAN APPENDIX 1B(4)

\section{INSTRUCTIONS:}

You may locate the server/drive name and path by taking the following step:

In Windows under Computer, right click on the Drive icon (e.g, F). Then click on Properties. The server/drive name and path will appear at the very top of the box.

If you need additional assistance contact your department computer support or system administrator for assistance in answering this question.

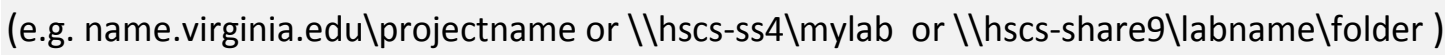

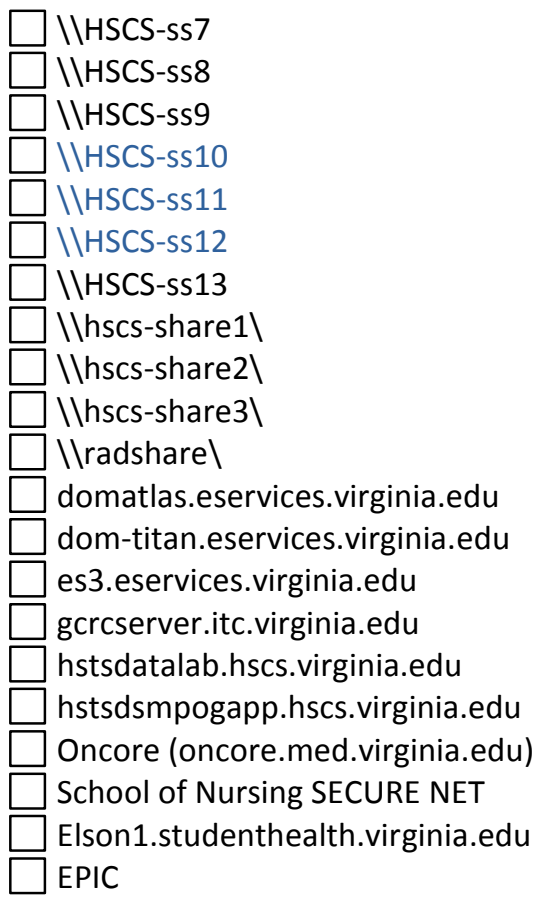

1B(5). $\square$ Directly to a server/drive managed by the sponsor or CRO in which data will be sent and stored in an encrypted fashion (e.g. must be shared and stored via Secure FX, Secure FTP, HTTPS, PGP) and the server/drive is configured to store data regulated by HIPAA.

IF CHECKED COMPLETE DATA SECURITY PLAN APPENDIX 1B(5)

1B(6). \Paper

Version Date: 8/26/2016

Page 4 of 23

IRB Submission \#10883 
1C. Will any data be stored electronically long term (e.g. during data analysis and beyond)? Yes $\bigotimes$ No $\square$

1C(1) IF YES, will it include storage of any health information or other sensitive data? Yes $\bigotimes$ No $\square$

1C(1)a If YES, check the HIPAA identifiers in the table below that will be kept with highly sensitive data in the same location (e.g. on the same electronic drive or in the same file).

\begin{tabular}{|c|c|c|}
\hline YES & NO & HIPAA Identifier \\
\hline & X & 1. Name \\
\hline & Х & $\begin{array}{l}\text { 2. Postal address information, other than town or city, state, and zip code ( e.g. street } \\
\text { name or GPS) }\end{array}$ \\
\hline & Х & 3. Age if over the age of 89 OR Date of Birth if over the age of 89 \\
\hline & $\triangle$ & 4. Telephone numbers \\
\hline & $\bar{X}$ & 5. Fax numbers \\
\hline & $\triangle$ & 6. Electronic mail addresses \\
\hline & $\triangle$ & 7. Social Security number- Must be checked if you are collecting SS\# for compensation. \\
\hline & & 8. Medical Record number \\
\hline & $\triangle$ & 9. Health plan beneficiary numbers \\
\hline & $\bar{\searrow}$ & $\begin{array}{l}\text { 10. Account numbers (e.g. bank numbers, credit card numbers, hospital bill account } \\
\text { number) }\end{array}$ \\
\hline & $\bigotimes$ & $\begin{array}{l}\text { 11. Certificate/license numbers (e.g. passport number, driver's license number, medical } \\
\text { board license number) }\end{array}$ \\
\hline & Х & 12. Vehicle identifiers and serial numbers, including license plate numbers \\
\hline & 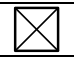 & 13. Device identifiers and serial numbers \\
\hline & $\triangle$ & 14. Web Universal Resource Locators (URLs) \\
\hline & $\bar{X}$ & 15. Internet Protocol (IP) address numbers \\
\hline & & 16. Biometric identifiers, including finger and voice prints \\
\hline $\bar{X}$ & & 17. Full face photographic images and any comparable images \\
\hline & $\bigotimes$ & $\begin{array}{l}\text { 18. Any other identifying number, characteristic, code that is derived from or related to } \\
\text { information about the individual (e.g. initials, last } 4 \text { digits of Social Security \#, mother's } \\
\text { maiden name, first } 3 \text { letters of last name.) } \\
\text { DO NOT CHECK THIS BOX IF THE SUBJECT ID WILL BE SOMETHING LIKE SUBJECT \#1. }\end{array}$ \\
\hline & $\bigotimes$ & $\begin{array}{l}\text { 19. Subject has rare disease or any other information that could identify them OR } \\
\text { Data is kept with a unique subject ID ( Subject \# 1) and the key to the code (Subject \# } 1= \\
\text { John Doe) is kept with the data. }\end{array}$ \\
\hline \multicolumn{3}{|c|}{$\begin{array}{l}\text { INSTRUCTIONS: If you checked YES to any HIPAA Identifier above your data is } \\
\text { considered to be HIGHLY SENSITIVE. } \\
\text { Follow requirements for handling Highly Sensitive data in the Privacy Plan of the } \\
\text { protocol. }\end{array}$} \\
\hline
\end{tabular}

Version Date: 8/26/2016

Page 5 of 23

IRB Submission \#10883 
$1 C(1) b$. Will you keep any of the following HIPAA identifiers in a different location from the data?

\begin{tabular}{|c|c|c|}
\hline YES & NO & HIPAA Identifier \\
\hline & & 7. Social Security number- Must be checked if you are collecting SS\# for compensation. \\
\hline & $\triangle$ & $\begin{array}{l}\text { 10. Account numbers (e.g. bank numbers, credit card numbers, hospital bill account } \\
\text { number) }\end{array}$ \\
\hline & $\triangle$ & $\begin{array}{l}\text { 11. Certificate/license numbers (e.g. passport number, driver's license number, } \\
\text { medical board license number) }\end{array}$ \\
\hline
\end{tabular}

INSTRUCTIONS: If you checked YES to any HIPAA Identifier above your data is considered to be HIGHLY SENSITIVE.

Follow requirements for handling Highly Sensitive data in the Privacy Plan of the protocol.

1C(2). WHERE will the data be stored long term (e.g. during data analysis and beyond)?

$\square$ Data will be stored in the same location to which it was collected or transferred as noted in 1A (Skip to Transferring Data)

If you did not answer either option above, check an applicable option below.

1C(2)a. $\square$ ONTO* an individual-use device (examples include desktop computer, smart phone app, flash (thumb) drive, external hard drive, tablet, laptop, $C D, C$ drive of your computer) )

- *ONTO means the data will reside on the device. Do not check this box if the device will simply be used to access a server.

If you checked YES to any item in $1 \mathrm{C}(1)$ a have you previously received ISPRO approval for storing data ONTO the same individual-use device? Yes $\square$ No If YES, submit ISPRO approval with this protocol submission. If NO, COMPLETE DATA SECURITY PLAN: APPENDIX 1C(2)a.

1C(2)b. $\square$ Web-based or cloud storage (e.g., UVaBox, UVa-Collab or other cloud service) If you checked YES to any item in 1C(1)a have you previously received ISPRO approval for storing data using the same web based system? Yes

If YES, submit ISPRO approval with this protocol submission.

If NO, COMPLETE DATA SECURITY PLAN: APPENDIX 1C(2)b. 
1C(2)c. $\triangle$ On a server NOT listed under 1C(2)d below.

Provide the name of the server/drive: \lab-fs08.mae.virginia.edu/NewData

\section{INSTRUCTIONS:}

You may locate the server/drive name and path by taking the following step: In Windows under Computer, right click on the Drive icon (e.g, F). Then click on Properties. The server/drive name and path will appear at the very top of the box. If you need additional assistance contact your department computer support or system administrator for assistance in answering this question.

(e.g. name.virginia.edu \myresearch or $\backslash \backslash \mathrm{hscs}-\mathrm{ss} 4 \backslash$ mylab or $\backslash \backslash \mathrm{hscs}$-share9 $\backslash$ mydata thisstudy )

- If you checked YES to any item in 1C(1)a have you previously received ISPRO approval for storing data ONTO the same server/drive? Yes $\square$ No $\square$

\section{If YES, submit ISPRO approval with this protocol submission.}

\section{If NO, COMPLETE DATA SECURITY PLAN: APPENDIX 1C2(c).}

$1 C(2)$ d. $\square$ Directly to one of the servers listed below. Check all applicable options and COMPLETE DATA SECURITY PLAN: APPENDIX IC(2)d.

\section{INSTRUCTIONS:}

You may locate the server/drive name and path by taking the following step: In Windows under Computer, right click on the Drive icon (e.g, F). Then click on Properties. The server/drive name and path will appear at the very top of the box. If you need additional assistance contact your department computer support or system administrator for assistance in answering this question.

(e.g. name.virginia.edu \projectname or $\backslash$ hsss-ss4 \mylab or $\backslash \backslash \mathrm{hscs}-$ share9/labname \folder

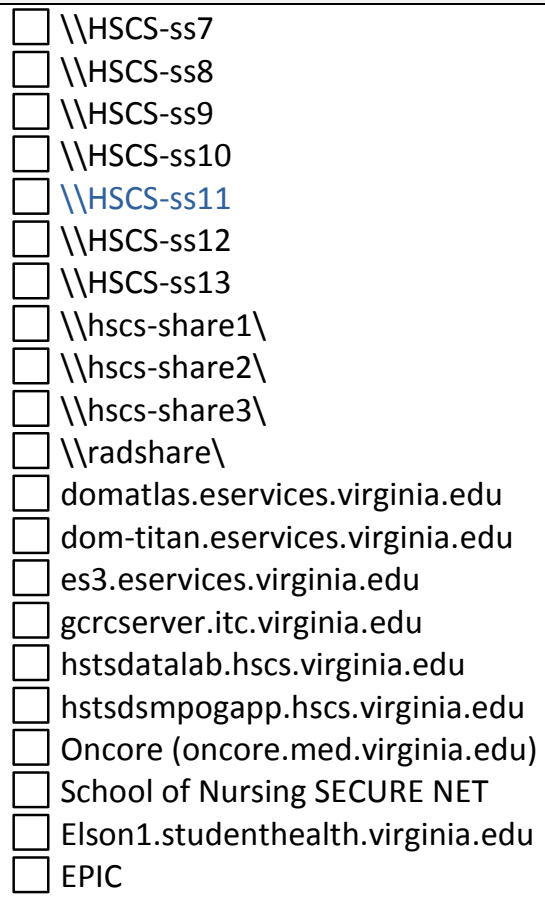

Version Date: 8/26/2016

Page 7 of 23

IRB Submission \#10883 
1C(2)e. $\square$ A server/drive managed by the sponsor or CRO in which the data will be sent and stored in an encrypted fashion (e.g. must be shared and stored via Secure FX, Secure FTP, HTTPS, PGP) onto a server/drive that is configured to store data regulated by HIPAA.

IF CHECKED, COMPLETE DATA SECURITY PLAN: APPENDIX IC(2)e.

IMPORTANT: If you checked any of the items $1 \mathrm{C}(2) \mathrm{a}-1 \mathrm{C}(2) \mathrm{c}$, submit ISPRO approval with new protocol submission.

You should consult with ISPRO during the development phase of this protocol if your protocol will involve highly technical issues such as the creation of a website to collect data, software application development, the use of a smart phone app, or if you plan to store identifiable data ONTO a tablet/laptop.

Otherwise submit the protocol and this Data Security Plan to ISPRO for review after the protocol is written.

ISPRO does NOT need the Protocol Cover Sheet, Consent Forms or any other ancillary documents. ISPRO CONTACT INFORMATION:

UVa Office of Information Security, Policy \& Records Office (ISPRO)

www.virginia.edu/ispro

Email: IT-Security@Virginia.edu 
1E(1) Will you be sharing/transferring data outside of UVa? Yes $\square$ No $\square$ If YES, Which of the following HIPAA identifiers will be shared/transported outside of UVa? If NO, COMPLETE THE APPENDIX SECTIONS THAT FOLLOW ONLY IF APPLICABLE.

\section{NOTE: No data collected under verbal consent/HIPAA authorization may be shared outside of UVa} with any of the following HIPAA identifiers.

\section{HIPAA Identifiers}

\begin{tabular}{|c|c|c|}
\hline YES & NO & \\
\hline & & 1. Name \\
\hline & & 2. Postal address information, other than town or city, state, and zip code \\
\hline & & 3. Age if over the age of 89 OR Date of Birth if over the age of 89 \\
\hline & & 4. Telephone numbers \\
\hline & & 5. Fax numbers \\
\hline & & 6. Electronic mail addresses \\
\hline & & 7. Social Security number- Must be checked if you are collecting SS\# for compensation. \\
\hline & & 8. Medical Record number \\
\hline & & 9. Health plan beneficiary numbers \\
\hline & & 10. Account numbers \\
\hline & & 11. Certificate/license numbers \\
\hline & & 12. Vehicle identifiers and serial numbers, including license plate numbers \\
\hline & & 13. Device identifiers and serial numbers \\
\hline & & 14 Web Universal Resource Locators (URLs) \\
\hline & & 15. Internet Protocol (IP) address numbers \\
\hline & & 16. Biometric identifiers, including finger and voice prints \\
\hline & & 17. Full face photographic images and any comparable images \\
\hline & & $\begin{array}{l}\text { 18. Any other unique identifying number, characteristic, code that is derived from or related to information about } \\
\text { the individual (e.g. initials, last } 4 \text { digits of Social Security \#, mother's maiden name, first } 3 \text { letters of last name.) }\end{array}$ \\
\hline & & $\begin{array}{l}\text { 19. Any other information that could be used alone or in combination with other information to identify an } \\
\text { individual. } \\
\text { (e.g. subject has rare disease, outside sponsor/institution has access to the data and a HIPAA identifier or the Key } \\
\text { to the Code e.g. Subject \# } 1 \text { = John Doe) } \\
\text { If the outside group only has access to the code (Subject \# } 1 \text { ) and not the key to the code (Subject \# 1= John Doe ) } \\
\text { DO NOT check this item. }\end{array}$ \\
\hline
\end{tabular}

Version Date: 8/26/2016

Page 9 of 23

IRB Submission \#10883 
1E(2). How will the data be shared/transported?

$\square$ Paper forms

If shipped outside of UVa must be shipped with tracking (FedEx, UPS, certified mail etc.)

Messenger mail not allowed if you have answered YES to any item above

$\square$ Email:

Not allowed if you have answered YES to any item above unless the data will only be sent to and from an individual with $a{ }^{*} H S$ in their email address

\section{Secure Email:}

Not allowed if you have answered YES to any item above UNLESS you use the HSC Mail System and follow these steps.

FAX:

Not allowed unless receiving fax machine is in a restricted-access location, the intended recipient is clearly indicated, and that recipient has been alerted to the pending transmission and is available to pick it up immediately. Also verify FAX numbers before faxing and use FAX cover sheet with a confidentiality statement.

$\square$ Devices such as flash-drive/ CD etc.:

Not allowed if you have answered YES to any item in $1 E(1)$ unless you written approval from a VP/ Dean. The request for their written approval should be obtained using the Highly Sensitive Data Storage Request Form. You may also contact the UVa Office of Information, Security, Policy and Records Management at IT-Security@Virginia.edu for assistance in completing this form.

Web Based Data Entry (e.g. website, database, registry): NOT Encrypted and Password Protected;

Not allowed if you have answered YES to any item 1E(1).

Web Based Data Entry (e.g. website, database, registry): Encrypted and Password Protected; If checked, do you confirm that you have verified with host site that the data will be sent and stored in an encrypted fashion (e.g. via Secure FX, Secure FTP, HTTPS, PGP)?

IF CHECKED COMPLETE DATA SECURITY PLAN APPENDIX 1B(5) if not already completed.

INSTRUCTIONS: Do not complete this section if the only data being shared/transported are being sent with specimens. See Specimens Section 
1. If sharing data with anyone outside of UVa do you confirm that you will obtain a contract/ material transfer agreement with them via the School of Medicine Grants and Contracts Office or the Office of Sponsored Programs (OSP) ospnoa@virginia.edu?

2. Will any data be sent outside of UVa to any person at another institution other than the sponsor or the FDA (e.g. researcher outside of UVa)?

Yes $\square$ No $\square$

INSTRUCTIONS:

If NO, skip questions $2 A-2 D$ below

2A. What will be shared?

2B. Who will it be shared with?

2C. What will they do with data?

2D.Will information be sent back to UVa?

If yes, please list what identifiers will be included and how it will sent back (e.g., by fax, secure email).

END OF FORM- COMPLETE THE APPENDIX SECTIONS THAT FOLLOW ONLY IF APPLICABLE. 


\section{Data Security Plan: APPENDIX 1B(1)}

1B(1). Collection of data ONTO* an individual-use device (examples include desktop computer, smart phone app, flash (thumb) drive, external hard drive, tablet, laptop, CD, Cdrive of your computer, camera, audio or video recorder)

$\bullet$

What kind of device is it (examples include desktop computer, smart phone app, flash (thumb) drive, external hard drive, tablet, laptop, $C D, C$ drive of your computer, camera, audio or video recorder) Video Recorder and Local Desktop Computer (Center for Applied Biomechanics) The data (kinematic) from subjects biking under different postures and resistances is videorecorded by Vicon motion tracking camera system. This Vicon camera system records motion of sensors and is not a typical video camera. If recognizable facial images are accidentally captured by reference video (via typical video camera from rear-oblique point-of-view), they will be blurred out. In addition, the recording is of someone riding a bicycle, which is not PHI. .

- Who manages / supports the device (e.g., Health Systems Computing Services (HS/CS), local computer support partner (LSP), self)? LSP: Meghan Darney

INSTRUCTIONS: If the device is managed/support by self you must follow both the setup and maintenance security standards described on the UVa Office of Information Security, Policy \& Records Office (ISPRO) webpage:

http://www.virginia.edu/informationsecurity/device-requirements.html

- How long will the data remain on the device before it is transferred elsewhere? Over the duration of the proposed investigation

- Will anyone other than study team members have access to data on the device?

Yes $\square$ No $\bigotimes$ If yes, describe:

- Will data be transferred elsewhere in an encrypted secure manner such as the use of SFTP or HTTPS? Yes $\$ No

- If yes, describe: Between External Hard Drive and Local Desktop Computer via USB transfer (unencrypted in USB)

- Are any backups made of the information on the device? Yes $\square$ No $\square$

- If yes, explain how backups are made and where they are stored:

- After information is transferred off the device will you securely delete all data from the device? Yes $\$ No $\square$

INSTRUCTIONS: For computers not using Windows 8 or newer, download and use the Secure Delete Program from ITS. If using Windows 8 or newer, click on Secure Delete when deleting a file. For Macintosh computers, select "Secure Empty Trash" from the Finder menu.

- Does the owner of the device (e.g. phone service provider/ app developer) have any rights to use or access data either individually or in aggregate? Yes $\square$ No $\square$

- If you are using an individual use device such as a camera or video recorder do you confirm the photos will not include the full face. Yes $\$ No $\square$

Version Date: 8/26/2016

Page 12 of 23

IRB Submission \#10883 
- If you are using a video or audio recorder, do you confirm the data will not include HIPAA identifiers? Yes $\bigotimes$ No

Version Date: 8/26/2016

Page 13 of 23

IRB Submission \#10883 


\section{Data Security Plan: APPENDIX 1B(2)}

1B(2.) Collection of data via web-based format (e.g. online consent, online surveys)

- Provide the web address (URL):

INSTRUCTIONS: (e.g., https://name1.name2.org/mystudy/login.html)

The URL is in the address bar of your web browser (e.g., Internet Explorer (IE), Firefox, Chrome)

If you need additional assistance contact your department computer support or system administrator for assistance in answering this question.

- How long will data remain on the website before it is transferred to a UVa server/drive or storage?

- Will anyone other than study team members have access to data on the non-UVa secure server/drive? Yes $\square$ No $\square$

- Will data be downloaded to a UVa secure server/drive in an encrypted secure manner such as the use of SFTP or HTTPS? Yes No $\square$

O If yes, describe:

- If checked provide the location:

INSTRUCTIONS : (e.g., https://name.hsc.virginia.edu) or \|hscs-ss1\

You may locate the server/drive name behind the drive designator by taking the following step:

In Windows under Computer, right click on the Drive icon (e.g F). Then click on Properties.

The server/drive name and path will appear at the very top of the box.

If you need additional assistance contact your department computer support or system administrator for assistance in answering this question.

- $\quad$ Are any backups made of the information on the secure server/drive? Yes $\square$ No $\square$ If yes, explain how backups are made and where they are stored:

- After information is transferred elsewhere will you securely delete all data from the website? Yes $\square$ No $\square$

- Do the owners of the website have any rights to use or access data either individually or in aggregate? Yes $\square$ No $\square$

- If the website not hosted at UVa, is there a Business Associates Agreement (BAA) with the provider of the non-UVa website? Yes No $\square$ 


\section{Data Security Plan: APPENDIX 1B(3)}

1B(3). To a server NOT listed under 1B(4) below.

Provide the name of the server/drive:

INSTRUCTIONS: You may locate the server/drive name and path by taking the following step:

In Windows under Computer, right click on the Drive icon (e.g, F). Then click on Properties. The server/drive name and path will appear at the very top of the box.

If you need additional assistance contact your department computer support or system administrator for assistance in answering this question.

(e.g. name.virginia.edu or \\home2.virginia.edu \research1)

-What kind of individual-use device will be used to connect to this server/drive?

(examples include desktop computer, smart phone app, flash (thumb) drive, external hard drive, tablet, laptop, CD, (drive of your computer) )?

-Who manages / supports this individual-use device (e.g., Health Systems Computing Services (HS/CS), local computer support person (LSP), self)?

- List how to contact this support:

INSTRUCTIONS: If the device is managed/support by self you must follow both the setup and maintenance security standards described on the UVa Office of Information Security, Policy \& Records Office (ISPRO) webpage:

http://www.virginia.edu/informationsecurity/device-requirements.html 


\section{Data Security Plan: APPENDIX 1B(4)}

1B(4). Directly to one of the servers listed below.

- What kind of individual-use device will be used to connect to this server/drive? (examples include desktop computer, smart phone app, tablet, laptop.)

- Who manages / supports this individual-use device (e.g., Health Systems Computing Services (HS/CS), local computer support person (LSP), self)?

- List how to contact this support:

INSTRUCTIONS: If the device is managed/support by self you must follow both the setup and maintenance security standards described on the UVa Office of Information Security, Policy \& Records Office (ISPRO) webpage:

http://www.virginia.edu/informationsecurity/device-requirements.html 


\section{Data Security Plan: APPENDIX 1B(5)}

1B(5). Directly to a server/drive managed by the sponsor or CRO in which data will be sent and stored in an encrypted fashion (e.g. must be shared and stored via Secure FX, Secure FTP, HTTPS, PGP) and the server/drive is configured to store data regulated by HIPAA.

Provide the name of the server/drive:

- What kind of individual-use device will be used to connect to this server/drive?

(examples include desktop computer, smart phone app, flash (thumb) drive, external hard drive, tablet, laptop, $C D, C$ drive of your computer) )?

- Who manages / supports this individual-use device (e.g., Health Systems Computing Services (HS/CS), local computer support person (LSP), departmental technology support group,, self)?

- List how to contact this support:

INSTRUCTIONS: If the device is managed/support by self you must follow both the setup and maintenance security standards described on the UVa Office of Information Security, Policy \& Records Office (ISPRO) webpage: http://www.virginia.edu/informationsecurity/devicerequirements.html 


\section{Data Security Plan: APPENDIX 1C(2)a}

1C(2)a. ONTO* an individual-use device (examples include desktop computer, smart phone app, flash (thumb) drive, external hard drive, tablet, laptop, CD, C drive of your computer) )

- What kind of device is it (e.g. desktop computer, smart phone app, flash (thumb) drive, tablet, laptop, $\mathrm{CD}, \mathrm{C}$ drive of your computer)

- Who manages / supports the device (e.g., Health Systems Computing Services (HS/CS), local computer support partner (LSP), self)?

INSTRUCTIONS: If the device is managed/support by self you must follow both the setup and maintenance security standards described on the UVa Office of Information Security, Policy \& Records Office (ISPRO) webpage: http://www.virginia.edu/informationsecurity/device-requirements.html

- How long with data remain on the device before it is transferred elsewhere?

Will anyone other than study team members have access to data on the device? Yes $\square$ No $\square$ If yes, describe:

- Will data be transferred elsewhere in an encrypted secure manner such as the use of SFTP or HTTPS? Yes $\square$ No $\square$

- If yes, describe:

- Are any backups made of the information on the device? Yes $\square$ No $\square$

- If yes, explain how backups are made and where they are stored:

- After information is transferred off the device will you securely delete all data from the device? Yes $\square$ No $\square$

INSTRUCTIONS: For computers not using Windows 8 or newer, download and use the Secure Delete Program from ITS. If using Windows 8 or newer, click on Secure Delete when deleting a file. For Macintosh computers, select "Secure Empty Trash" from the Finder menu.

- Does the owner of the device (e.g. phone service provider/ app developer) have any rights to use or access data either individually or in aggregate? Yes No 


\section{Data Security Plan: APPENDIX 1C(2)b}

1C(2)b. Web-based or cloud storage (e.g., UVaBox, UVa-Collab or other cloud service)

- Provide the name of the website or cloud storage:

- Who manages / supports this individual-use device (e.g., Health Systems Computing Services (HS/CS), local computer support person (LSP), self)?

- List how to contact this support:

INSTRUCTIONS: Not allowed if you have answered YES to any HIPAA identifier except the use of a unique subject ID (e.g. Subject \# 1).

NOTE: No research data of any kind may be stored in a non-UVa licensed cloud provider such as Dropbox, Google Drive, SkyDrive, Survey Monkey etc.

- What kind of individual-use device will be used to connect to this service? (e.g. laptop, tablet, desktop computer)?

- Who manages / supports this individual-use device (e.g., Health Systems Computing Services (HS/CS), local computer support person (LSP), self)?

○ List how to contact this support:

INSTRUCTIONS: If the device is managed/support by self you must follow both the setup and maintenance security standards described on the UVa Office of Information Security, Policy \& Records Office (ISPRO) webpage: http://www.virginia.edu/informationsecurity/devicerequirements.html 


\section{Data Security Plan: APPENDIX 1C(2)c}

1C(2)c. To a server NOT listed.

Provide the name of the server/drive: \|cab-fs08.mae.virginia.edu\NewData

- What kind of individual-use device will be used to connect to this server/drive? (examples include desktop computer, smart phone app, flash (thumb) drive, external hard drive, tablet, laptop, $C D, C$ drive of your computer)? Local Laptop Computer

- Who manages / supports this individual-use device (e.g., Health Systems Computing Services (HS/CS), local computer support person (LSP), self)? LSP: Mark Mccardell

- List how to contact this support: 434-297-8023, mam3wn@virginia.edu

INSTRUCTIONS: If the device is managed/support by self you must follow both the setup and maintenance security standards described on the UVa Office of Information Security, Policy \& Records Office (ISPRO) webpage:

http://www.virginia.edu/informationsecurity/device-requirements.html 


\section{Data Security Plan: APPENDIX 1C(2)d}

$\mathbf{1 C ( 2 ) d . ~ D i r e c t l y ~ t o ~ o n e ~ o f ~ t h e ~ s e r v e r s ~ l i s t e d . ~}$

- What kind of individual-use device will be used to connect to this server/drive? (examples include desktop computer, smart phone app, tablet, laptop.)

- Who manages / supports this individual-use device (e.g., Health Systems Computing Services (HS/CS), local computer support person (LSP), self)?

- List how to contact this support:

INSTRUCTIONS: If the device is managed/support by self you must follow both the setup and maintenance security standards described on the UVa Office of Information Security, Policy \& Records Office (ISPRO) webpage:

http://www.virginia.edu/informationsecurity/device-requirements.html 


\section{Data Security Plan: APPENDIX 1C(2)e}

1C(2)e. Directly to a server/drive managed by the sponsor or CRO in which data will be sent and stored in an encrypted fashion (e.g. must be shared and stored via Secure FX, Secure FTP, HTTPS, PGP) and the server/drive is configured to store data regulated by HIPAA.

Provide the name of the server/drive:

- What kind of individual-use device will be used to connect to this server/drive? (examples include desktop computer, smart phone app, flash (thumb) drive, external hard drive, tablet, laptop, $C D, C$ drive of your computer) )?

- Who manages / supports this individual-use device (e.g., Health Systems Computing Services (HS/CS), local computer support person (LSP), departmental technology support group,, self)?

- List how to contact this support:

INSTRUCTIONS: If the device is managed/support by self you must follow both the setup and maintenance security standards described on the UVa Office of Information Security, Policy \& Records Office (ISPRO) webpage: http://www.virginia.edu/informationsecurity/devicerequirements.html 


\section{Data Security Plan Glossary:}

Data Collected or Received: Where you put any kind of data recorded or gathered from another source for purposes of research. The data can come from any source, electronic, paper or voice. You may be sent these individual data points by paper, subject/patient interview or electronically. You may be manually extracting these data points from EPIC. You may be collecting these data with devices (camera, heart monitor, etc.)

Data Stored Long Term (Data storage) is different from data collected as it implies a longer-term nonvolatile storage. It may be the same location as collected, (such as paper or HSCS server) or it may be a new location (computer drive or paper). It is where it is located for further analysis, manipulation and access.

Highly Sensitive Data: includes personal information that can lead to identity theft if exposed and/or health information that reveals an individual's health condition and/or history of health services use. Electronic data storage policy: http://uvapolicy.virginia.edu/policy/IRM-015 Three HIPAA-identifiers are highly sensitive data themselves. These are \#7-Social Security Number, \#10Account numbers, if it's a financial account number such as credit card or bank card number and \#11 Certificate/license number if it's a passport number, driver's license number, board license number, etc.). If these are in a file without any personal health information (PHI) it is still highly sensitive data (HSD).

Moderately Sensitive Data: includes information that is not highly sensitive nor is intentionally made public. So this category includes most of the data and information we work with. All research data that is not intentionally made public (e.g., published) is considered moderately sensitive data (MSD).

Individual Use Device: any kind of technology that has persistent memory. Flash memory, Solid State drives, traditional hard drives, SD cards, USB thumb drives (sticks) allow for data to be kept long term. This means that any smartphones, laptops, tablets, biometric fitness devices and digital cameras and MP3 recorders (digital audio) qualify as individual use devices that could store potential data and must be protected.

Web based or Cloud storage: generally implies a storage server where a web browser is the main way to login and manipulate files. Sometimes a smartphone app is created to interface to these cloud storage containers. Examples include UVaBox, Google Drive, Google Docs, DropBox. Use of any Google Drive, Doc, Email, etc. for any UVa data or files is against UVa data protection policies. 


\section{UIIIIIIVERSTTY of VIRGINIA CENTER FOR APPLIED BIOMECHANICS}

\section{Parametric Analysis of Fatigue in Stationary Biking: A Research Study}

We are familiar with impact-based sports injury, but when it comes to fatigue-based injury, how much fatigue is too much? By measuring how stationary bikers move and exert forces as they get tired, we can lay a foundation for answering this question. Our study aims to use this data to explain the dynamic relationship between muscle fatigue and biking forces so that injury prediction models can be developed.

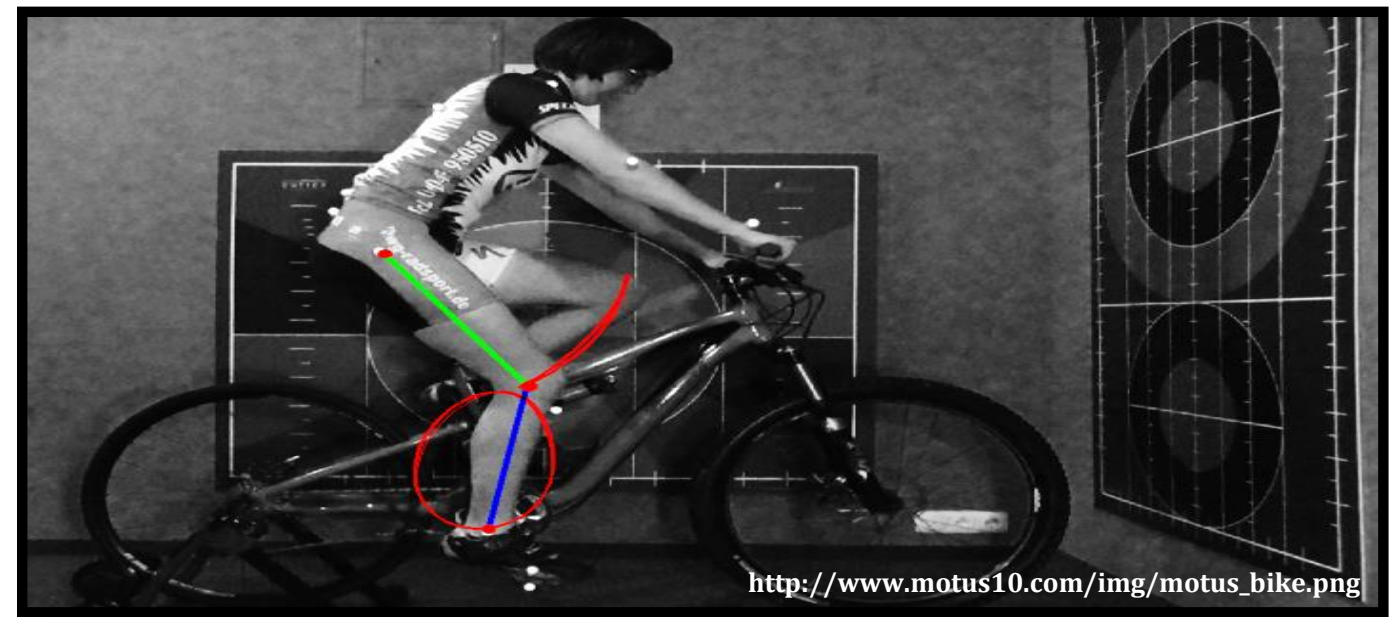

Study Procedure

1. Arrive at Center

2. Stretch \& Warm-Up

3. Endurance Ride

4. Refreshments \& Departure

LENGTH OF STUDY

\section{COMPENSATION}

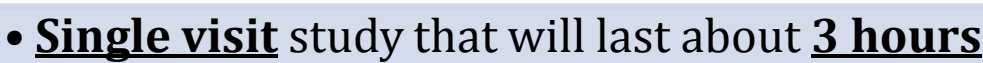

- Available Test Dates: Weekdays, October 1 - 31, 2016

- Volunteers will be compensated via VISA Gift Card

- 2-3 years of biking experience

- Adult between 20 and 45 years old

- Between 5' 3" and 6' 6" in height

- Under 250lbs in weight

- English Speaking

- No known pregnancy

- No known heart disease (cardiomyopathy), stroke or high blood pressure

- No known current or ongoing physical injury

- Other Restrictions may apply

- For more information, email: Biking Study Research Team, ds7rs@virginia.edu 


\section{IIIINIVERSITY of VIRGINIA \\ CENTER FOR APPLIED BIOMECHANICS \\ Parametric Analysis of Fatigue in Stationary Biking: A Computational Approach}

Email Recruitment

Dear

\begin{tabular}{|l|l|l|}
\hline & \multicolumn{2}{|c|}{ UVA IRB-HSR } \\
\hline
\end{tabular}

Are you interested in participating in a scientific study that involves biking? The University of Virginia's Center for Applied Biomechanics is conducting a research study that aims to understand how riders' performance changes as they fatigue during high intensity stationary biking in hopes of relating the observed behavior changes to the potential for long-term injury risk.

As part of the study, volunteers will be asked to ride a stationary bike at a target speed while the resistance level is increased in steps from "easy" to "high" during which the rider will attempt to maintain a consistent target speed as long as possible during the endurance ride.

For your safety and scientific rigor of the study you must:

- Have 2-3 of recreational/competitive biking experience

- Be between 5'4" and 6'6"

- Weigh less than 250lbs.

- Have no known heart disease (cardiomyopathy)

- Have no history of irregular heart beat

- Have no history of stroke

- Have no history of high blood pressure

- Have no know current or ongoing physical injury*

*Any health condition deemed to place you at risk as determined by your physician.

Volunteers will be compensated for their time via VISA gift card, and the 3-hour time slots are fairly flexible on weekdays throughout the month of October 2016.

If you are interested in knowing more about this research volunteer opportunity, please contact the research investigators at $\underline{\text { ds7rs@ virginia.edu. }}$

Please be aware that additional screening criteria exist. Complete study details will be disclosed in subsequent information emails from the research team after you have expressed interest.

Thank you!

Biking Study Research Team

Center for Applied Biomechanics

University of Virginia

Charlottesville, VA 22911 


\section{IIIIUIVERSITY of VIRGINIA \\ CENT'TR FOR APPLIED BIOMECHANICS \\ Parametric Analysis of Fatigue in Stationary Biking: A Computational Approach}

Facebook Recruitment

Do you love to bike? Want to offer your

\begin{tabular}{|l|l|l|}
\hline & \multicolumn{2}{|c|}{ UVA IRB-HSR } \\
\hline
\end{tabular}
time to help scientific research?

The University of Virginia's Center for Applied Biomechanics is currently recruiting experienced recreational and competitive bikers to volunteer in a study that aims to understand how bikers' performance changes as they fatigue during high intensity stationary biking. Volunteers will be compensated for their time via VISA gift card, and time slots are fairly flexible on weekdays throughout the month of October 2016. If you are interested in knowing more about the research volunteer opportunity, please contact the research investigators at ds7rs@virginia.edu. Thank you! 


\section{Pre-Screen Questions}

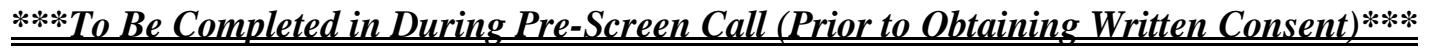

Make sure you have the current IRB-stamped consent form. Orally review the items below and any other information about the study with the subject or that their family wishes to discuss.

\section{Confirm Potential Volunteer Identity:}

Potential Volunteer Name:
First Name
Middle Initial
Last Name

General purpose of study - to determine the safety and effectiveness of exercising after reaching a certain level of fatigue. The data from the EMG, load cells, and motion capture will give us the opportunity to study the effects of fatigue and how they relate to a person's risk for injury. We aim to understand the dynamics of how the human body operates as it gets more and more tired.

Note that enrollment in the trial is purely voluntary and that the patient can withdraw at anytime. Their choice will not affect their usual care at UVA.

Inclusion and Exclusion criteria (in lay terms) - Adults with known heart disease (e.g. cardiomyopathy), history of irregular heart been, stroke or hypertension or those with recent or non-properly healed injuries, pregnancy, non-fluency in the English language, or lack of riding experience will be excluded from the study.

Adults (age 20-45) who have 2-3 years of experience bike riding on either a competitive level or are in a riding team or club may be included in this study. You must be between the heights of 5' 3" and 6' 4" to properly fit on our bike.

Brief review of subject visits - During your stay you will be provided with athletic shorts and a shirt (you must provide socks/shoes and undergarments) and you are to change into them upon arrival. You will be guided through about 5-10 minutes of stretching and be given an additional 5 minutes to stretch on your own. You will have a 5 minute guided warm up ride on the bike, and then you will be hydrated and fitted with EMG electrodes and the markers for the motion capture. You may be asked to shave off hair from the area where the electrode patches will be placed. You will then be guided to ride for about 20 to 30 minutes of intense pedaling. You will be monitored during this process and measurements will be taken until you have reached a certain level of fatigue. "Fatigue" is defined in this study as dropping below 30rpm after maintaining 60-75rpm at "high" resistance. You, the rider will set your own resistance levels to your ability. When the testing is done, you will be assisted off the bike, followed by provided light snacks and refreshments. After confirming expected data quality, we will remove all sensors and markers, and ask that you change into a spare set of clothes. Ice-packs will be provided to help relieve soreness resulting from your session. At this point, you are free to leave the center premises.

There is only one 3-hour visit, so no follow-up visit is required.

Discuss the risks/alternatives/benefits of the study -

$\begin{array}{ll}\text { Likelv Risks } & \text { Less Likelv Risks: } \\ \text { - Fatigue } & - \text { Joint Pain } \\ \text { - Shortness of Breath } & - \text { Moderate-Severe Muscle } \\ \text { - Perspiration } & \text { Discomfort } \\ \text { - Light Muscle Soreness } & - \text { Dehydration } \\ \text { - Skin irritation or } & \\ \quad \text { abrasion } & \end{array}$
Rare, but Serious Risks:
- Muscle Tear
- Joint Sprain
- Bone Fracture
- Heart Complications
- Moderate-Severe Dehydration
- Joint Pain


Discuss study payment/cost to subject and HIPAA language-You will be compensated for your time by participating in this study via VISA gift card. There is no cost for participating in this study. People conducting and reviewing the study will see your personal information, but they will not share it when the study data is published.

\section{Ask questions (Please Note YES/NO Responses Below):}

\section{$\underline{\text { YES }} \quad \underline{\text { NO }}$}

1. $\square \quad \square \quad$ Are you between 20 and 45 years old?

2. $\square \quad \square \quad$ Are you under 250lbs in weight?

3. $\square \quad \square \quad$ Are you inclusively between 5'4" and 6'6" tall?

4. $\square \quad \square \quad$ Are you comfortable answering a few questions about medical history/injuries?

IF NO, Please continue to Questions 5-7:

5a. $\square \quad \square \quad$ Do you have any current or ongoing physical injuries?

5b. $\square \quad \square \quad$ Do you have any known history of heart disease (cardiomyopathy) irregular heart beat, stroke or high blood pressure?

5c $\square \quad \square \quad$ Are you pregnant?

$6 \square \quad \square \quad$ Do you have 2-3 years of recreational or competitive biking experience?

\section{Concluding Question:}

$7 \square \quad \square \quad$ Do you have any questions or concerns at this time?

If there are any questions/concerns, please indicate them here.

\section{Keep the original for the research records}

$\underline{\text { You should note responses electronically in the research charts (encrypted excel spreadsheet) }}$ 


\section{Consent of an Adult to Be in a Research Study}

In this form "you" means a person 18 years of age or older who is being asked to volunteer to participate in this study.

\section{Participant's Name}

\begin{tabular}{|l|l|}
\hline Principal Investigator: & Dr. Matthew B. Panzer \\
& $\begin{array}{l}\text { 4040 Lewis and Clark Drive, Charlottesville, VA } 22911 \\
\text { (434) 297-8062 }\end{array}$ \\
\hline
\end{tabular}

\section{What is the purpose of this form?}

This form will provide you with information about this research study. You do not have to be in the study if you do not want to. You should have all your questions answered before you agree to be in this study.

Please read this form carefully. If you want to be in the study, you will need to sign this form. You will be given a signed copy of this form.

\section{Who is funding this study?}

This study is funded by the UVA-internal Double Hoo Research Grant. Study resources are provided by the UVA Center for Applied Biomechanics.

\section{Why is this research being done?}

The purpose of this study is to develop a mathematical model for predicting fatigue-induced injury risk for biking to help guide training strategies. The goal is use this data to find the dynamic relationship between fatigue and risk of injury.

As personal fitness becomes increasingly prevalent in society, a focus on maintaining safe exercise technique is crucial to avoid unintended injury. Stationary biking, or spinning, is one particular exercise that has become very popular in the past decade. Spinning is commonly done in large group settings without prior individual training, therefore leaving the intensity and duration of spinning sessions primarily unmonitored. Rider feedback and stories of spinning-related injuries to the lower-back and knees suggest that such injuries may be due to improper exercise technique. We would like to determine the effects of fatigue on rider performance and better understand the basis for long-term biking injury.

Up to 15 people will be in this study at UVA.

\section{What will happen if you are in the study?}

If you agree to be in this study, you will sign this consent form before any study related procedures take place.

Version Date: 08/30/16

Page Number: 1 of 7

\begin{tabular}{|l|l|l|}
\hline \multirow{2}{*}{\begin{tabular}{|l|}
$|c|$ \\
\hline
\end{tabular}} & \multicolumn{2}{|c|}{ UVA IRB-HSR } \\
\cline { 2 - 3 } & Approval Date: & $09-08-16$ \\
\cline { 2 - 3 } & Expiration Date: & $09-7-17$ \\
\hline
\end{tabular}


IRB-HSR \# 19248

Parametric Analysis of Fatigue in Stationary Biking: A Computational Approach

Procedures (All are conducted to help research goals):

- In a private area you will change into special clothing for the study.

- You will answer questions to find out if you are physically able to do the study procedures.

- You will complete a 5-minute guided stretch, followed by 5 minutes of free stretching until you are comfortable for physical activity.

- Proper adjustments to the stationary bike will be made to fit your body.

- You will do a guided 5 minute warm up ride on the bike (data will not be collected)

- You will drink 8oz. of water after stretching and after the warmup ride

- 12 surface EMG electrodes will attached to the lower back, abdomen, and legs. If hair is interfering with the electrode patch you may be asked to shave the hair off in order to place the patch.

- 35 position markers will be placed on your clothing for motion tracking

- You will ride the stationary bike for around 20 to 30 minutes.

- While this experiment is running, you will undergo EMG muscle activation sensing, motion tracking, and force distribution on the pedals and seat. (Data will be collected)

- Biking will be stopped when a predetermined level of fatigue is reached. "Fatigue" is defined as dropping below 30rpm after maintaining 60-75rpm at "high" resistance. You, the rider, will set your own resistance levels to your ability.

- If needed Ice packs will be provided to you for your sore muscles.

- You will be given more water and light snacks before leaving.

\section{WHAT ARE YOUR RESPONSIBILITIES IN THE STUDY?}

You have certain responsibilities to help ensure your safety.

These responsibilities are listed below:

- You must not eat or drink any sort of caffeinated or energy boosting substances during or within 5 hours before the course of your participation in this study (Energy Drinks, 5 Hour Energy, coffee, caffeinated chocolate, etc.)

- Last "full meal" must be between 2-3 hours before arriving at study site.

- You must provide your own transportation to the study visit.

- You must bring your own shoes and undergarments for the testing. (Shorts and shirts will be provided)

- You must be completely truthful about your health history.

- Follow all instructions given.

- You should tell the research investigators or study staff about any changes in your health or you are experiencing unexpected or unusual physical discomfort during the study.

- Answer all of the study-related questions completely.

\section{How long will this study take?}

Your participation in this study will require one study visit over 2-3 hours.

Version Date: 08/30/16

Page Number: 2 of 7 
If you want to know about the results before the study is done:

The final results of the research will not be known until all the information from everyone is combined and reviewed. At that time, study results will be released to participants who requested information at the start of this study with a valid email address.

\section{What are the risks of being in this study?}

Risks and side effects related to the procedure (Riding the bike) include:

Likely

- Fatigue

- Shortness of Breath

- Perspiration

- Light Muscle Soreness

- Skin irritation or minor abrasion from shaving skin

Less Likely

- Joint Pain

- Moderate to Severe Muscle Discomfort

Rare but Serious

- Light Dehydration

- Muscle Tear/Strain

- Joint Sprain

- Bone fracture

- Heart Complications

- Moderate to Severe Dehydration

\section{Risks of Videotaping:}

You will be video taped in reference to the motion capture data. Your face may be shown. The only people with access to this footage will be the investigators. Footage is to be stored indefinitely on secure UVA devices. No audio will be recorded.

\section{Could you be helped by being in this study?}

You will not be helped by being in this study.

\section{What are your other choices if you do not join this study?}

The only other choice is not to be in this study.

If you are a patient at UVa your usual care will not be affected if you decide not to participate in this study.

If you are an employee of UVa your job will not be affected if you decide not to participate in this study. 
IRB-HSR \# 19248

Parametric Analysis of Fatigue in Stationary Biking: A Computational Approach

\section{Will you be paid for being in this study?}

You will be paid $\$ 50.00$ for finishing this study by VISA gift card.

You should get your payment about immediately after finishing the study session. The income may be reported to the IRS as income.

You will not be paid if it is determined that it is not safe for you to do the study procedures. You will be paid the full amount for your participation if the study leader stops the testing due to health reasons during the study procedures.

\section{Will being in this study cost you any money?}

You will be responsible for the cost of travel to come to any study visit at the Center for Applied Biomechanics, and for the cost of shoes and undergarments for testing. (Shirtsand shorts will be provided)

All of the procedures in this study are being done for research purposes and will be provided at no cost to you or your health insurance:

You and/or your insurance company must pay for any tests or care given beyond what is required in this study. In addition, you and/or your health insurance may also have to pay for other drugs or treatments that are given to help you control any side effects. You will have to pay for any costs not covered by your health plan. You may be responsible for any co-payments or deductibles. You may wish to ask for an estimate of your financial costs. You may also wish to check with your insurance company before the study starts. Ask what they will cover and if they require you to get their permission before you decide to be in the study.

\section{What if you are hurt in this study?}

If you are hurt as a result of being in this study, there are no plans to pay you for medical expenses, lost wages, disability, or discomfort. The charges for any medical treatment you receive will be billed to your insurance. You will be responsible for any amount your insurance does not cover. You do not give up any legal rights, such as seeking compensation for injury, by signing this form.

\section{What happens if you leave the study early?}

You can change your mind about being in the study any time. You can agree to be in the study now and change your mind later. If you decide to stop, please tell us right away. You do not have to be in this study to get services you can normally get at the University of Virginia.

Even if you do not change your mind, the study leader can take you out of the study. Some of the reasons for doing so may include:

a) The study leader closes the study for safety, administrative, or other reasons

Version Date: 08/30/16

Page Number: 4 of 7 
b) Instructions from the investigators are not be followed

c) Equipment failure

d) You no longer meet the requirements to be in the study

\section{How will your personal information be shared?}

The UVa researchers are asking for your permission to gather, use, and share, information about you for this study. If you decide not to give your permission, you cannot be in this study, but you can continue to receive regular medical care at UVA.

\section{If you sign this form, we may collect any or all of the following information about you:}

- Personal information such as name, address and date of birth

o Your health information for this study.

\section{Who will see your private information?}

- The researchers to make sure you are compatible with experiment requirements, observe the effects of the study, and understand its results.

- People or groups that oversee the study to make sure it is done correctly.

- Tax reporting offices (if you are paid for being in the study).

- People who evaluate study results, which can inclu de sponsors.

- If you tell us that someone is hurting you, or that you might hurt yourself or someone else, the law may require us to let people in authority know so they can protect you and others.

Some of the people outside of UVa who may see your information may not have to follow the same privacy laws that we follow. They may release your information to others, and those laws may no longer protect it.

The information collected from you might be published in a medical journal. This would be done in a way that protects your privacy. No one will be able to find out from the article that you were in the study.

Version Date: 08/30/16

Page Number: 5 of 7 


\section{What if you sign the form but then decide you don't want your private information shared?}

You can change your mind at any time. Your permission does not end unless you cancel it. To cancel it, please send an email to the researchers listed on this form. Then you will no longer be in the study. The researchers will still use information about you that was collected before you ended your participation.

\section{Please contact the researchers listed below to:}

- Obtain more information about the study

- Ask a question about the study procedures or treatments

- Report an illness, injury, or other problem (you may also need to tell your regular doctors)

- Leave the study before it is finished

- Express a concern about the study

Principal Investigator: Matthew Panzer

4040 Lewis and Clark Drive, Charlottesville, VA 22911

Telephone: (434)297-8062

Email Address: $\underline{\text { mbp2@virginia.edu }}$

Sub-Investigator: Deepak Sathyanarayan

Telephone: (386)366-0547

Email Address: ds7rs@virginia.edu

Study Coordinator: Austin Rivera

Telephone: (609) 781-7766

Email Address: atr8ec@virginia.edu

\section{What if you have a concern about this study?}

You may also report a concern about this study or ask questions about your rights as a research subject by contacting the Institutional Review Board listed below.

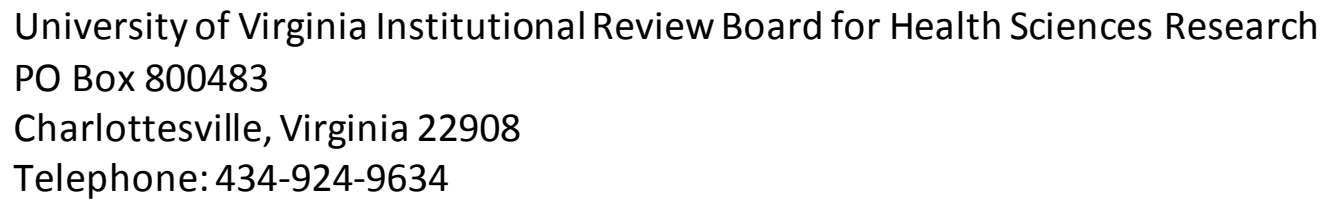

When you call or write about a concern, please give as much information as you can. Include the name of the study leader, the IRB-HSR Number (at the top of this form), and details about the problem. This will help officials look into your concern. When reporting a concern, you do not have to give your name.

Version Date: 08/30/16

Page Number: 6 of 7 
IRB-HSR \# 19248

Parametric Analysis of Fatigue in Stationary Biking: A Computational Approach

\section{Signatures}

What does your signature mean?

Before you sign this form, please ask questions about any part of this study that is not clear to you. Your signature below means that you have received this information and all your questions have been answered. If you sign the form it means that you agree to join the study. You will receive a copy of this signed document.

\section{Consent From Adult}

$\overline{\text { PARTICIPANT (SIGNATURE) }} \quad \overline{\text { PARTICIPANT (PRINT) }} \overline{\text { DATE }}$

To be completed by participant if 18 years of age or older.

\section{Person Obtaining Consent}

By signing below you confirm that you have fully explained this study to the potential subject, allowed them time to read the consent or have the consent read to them, and have answered all their questions.

\begin{tabular}{lll}
\hline $\begin{array}{l}\text { PERSON OBTAINING CONSENT } \\
\text { (SIGNATURE) }\end{array}$ & $\begin{array}{l}\text { PERSON OBTAINING CONSENT } \\
\text { (PRINT) }\end{array}$ & $\overline{\text { DATE }}$
\end{tabular}

Version Date: 08/30/16

Page Number: 7 of 7 


\section{Informed Consent Comprehension Overview}

\section{***To Be Completed in Parallel with Obtaining Written Consent****}

Make sure you have the current IRB-stamped consent form. Orally review the items below and any other information about the study with the subject or that their family wishes to discuss.

General purpose of study - to determine the safety and effectiveness of exercising after reaching a certain level of fatigue. The data from the EMG, load cells, and motion capture will give us the opportunity to study the effects of fatigue and how they relate to a person's risk for injury. We aim to understand the dynamics of how the human body operates as it gets more and more tired.

Note that enrollment in the trial is purely voluntary and that the patient can withdraw at anytime. Their choice will not affect their usual care at UVA.

Inclusion and Exclusion criteria (in lay terms) - Adults with known heart disease (e.g .cardiomyopathy) history of irregular heart been, stroke or hypertension or those with recent or non-properly healed injuries, pregnancy, nonfluency in the English language, or lack of riding experience will be excluded from the study.

Adults (age 20-45) who have 2-3 years of experience bike riding on either a competitive level or are in a riding team or club may be included in this study. You must be between the heights of 5' 3"' and 6' 4" to properly fit on our bike.

Brief review of subject visits - During your stay you will be provided with athletic shorts and a shirt (you must provide socks/shoes and undergarments) and you are to change into them upon arrival. You will be guided through about 5-10 minutes of stretching and be given an additional 5 minutes to stretch on your own. You will have a 5 minute guided warm up ride on the bike, and then you will be hydrated and fitted with EMG electrodes and the markers for the motion capture. You may have to shave some hair from the area where the electrode patches will be placed. You will then be guided to ride for about 20 to 30 minutes of intense pedaling. You will be monitored during this process and measurements will be taken until you have reached a certain level of fatigue. "Fatigue" is defined in this study as dropping below 30rpm after maintaining 60-75rpm at "high" resistance. You, the rider will set your own resistance levels to your ability. When the testing is done, you will be assisted off the bike, followed by provided light snacks and refreshments. After confirming expected data quality, we will remove all sensors and markers, and ask that you change into a spare set of clothes. Ice-packs will be provided to help relieve soreness resulting from your session. At this point, you are free to leave the center.

In the event of a health emergency, your research coordinators are CPR/AED trained and will act promptly to call 91-1 and deliver immediate care until emergency personnel arrive. Approximate minimum time from 9-1-1 contact to hospital arrival is 11 minutes and 30 seconds. Maximum 38 minutes and 30 seconds if UVA Hospital is required.

There is only one 3-hour visit, so no follow-up visit is required.

Discuss the risks/alternatives/benefits of the study -

$\begin{array}{ll}\text { Likelv Risks } & \text { Less Likelv Risks: } \\ \text { - Fatigue } & - \text { Joint Pain } \\ \text { - Shortness of Breath } & - \text { Moderate-Severe Muscle } \\ \text { - Perspiration } & \text { Discomfort } \\ \text { - Light Muscle Soreness } & - \text { Dehydration } \\ \text { - Skin irritation or } & \\ \quad \text { abrasion } & \end{array}$

Rare, but Serious Risks:

- Muscle Tear

- Joint Sprain

- Bone Fracture

- Heart Complications

- Moderate-Severe Dehydration

- Joint Pain 
Discuss study payment/cost to subject and HIPAA language -You will be compensated for your time by participating in this study via VISA gift card. There is no cost for participating in this study. People conducting and reviewing the study will see your personal information, but they will not share it when the study data is published.

\section{Ask questions (Please Note Responses Below):}

Question: Please tell me what you understand about what you need to do in this study.

Answer:

Question: What follow-up is required for this study?

Answer:

Question: Are there risks if you choose to be in the study?

Answer:

Question: What would you do if you wanted to quit being in this study?

Answer:

Proceed to signing the consent form and making 2 copies of it. (Give one copy to the study participant, and keep the original for the research records.)

You should note in the research charts (encrypted excel spreadsheet): "Subject was given a copy of the informed consent form to read. The subject was given an opportunity to ask questions and have those questions answered. The key points of the consent were reviewed orally with the subject. The subject appeared to understand what is involved in the study. The subject agreed to participate in the study, personally signed and dated the informed consent form and was given a copy of the signed form prior to any study-related procedure being performed."

If there are any discrepancies, please indicate them here. 
Parametric Analysis of Fatigue in Stationary Biking: A Computational Approach

\section{Volunteer Survey}

***To Be Completed in After Obtaining Written Consent***

\section{Personal Information}

Name:

Address:

Primary Phone \#: (___ $)$ -

Height:

Hip to Heel Length:
Age:

Date:

City/State/Zip:

Primary Email:

lbs.

Weight:

in. (Measure from the top of your hip bone to the heel of your shoe)

\section{Medical History and Injuries}

Injuries and Approx. Date:

How were they treated?

Was treatment successful (you can ride a bike essentially pain free)?

If not, what types of motions cause discomfort?

Medical Conditions:

Current Medications/Treatments:

\section{Competition}

At what level and frequency do you compete or ride? Collegiate? Mileage per Race?

Current Team/Club(s): 


\section{Appendix D Engineering Drawings}

The following pages present dimensioned CAD drawings created in SolidWorks to guide the construction of custom instrumented pedal and seat fixtures for this study. 


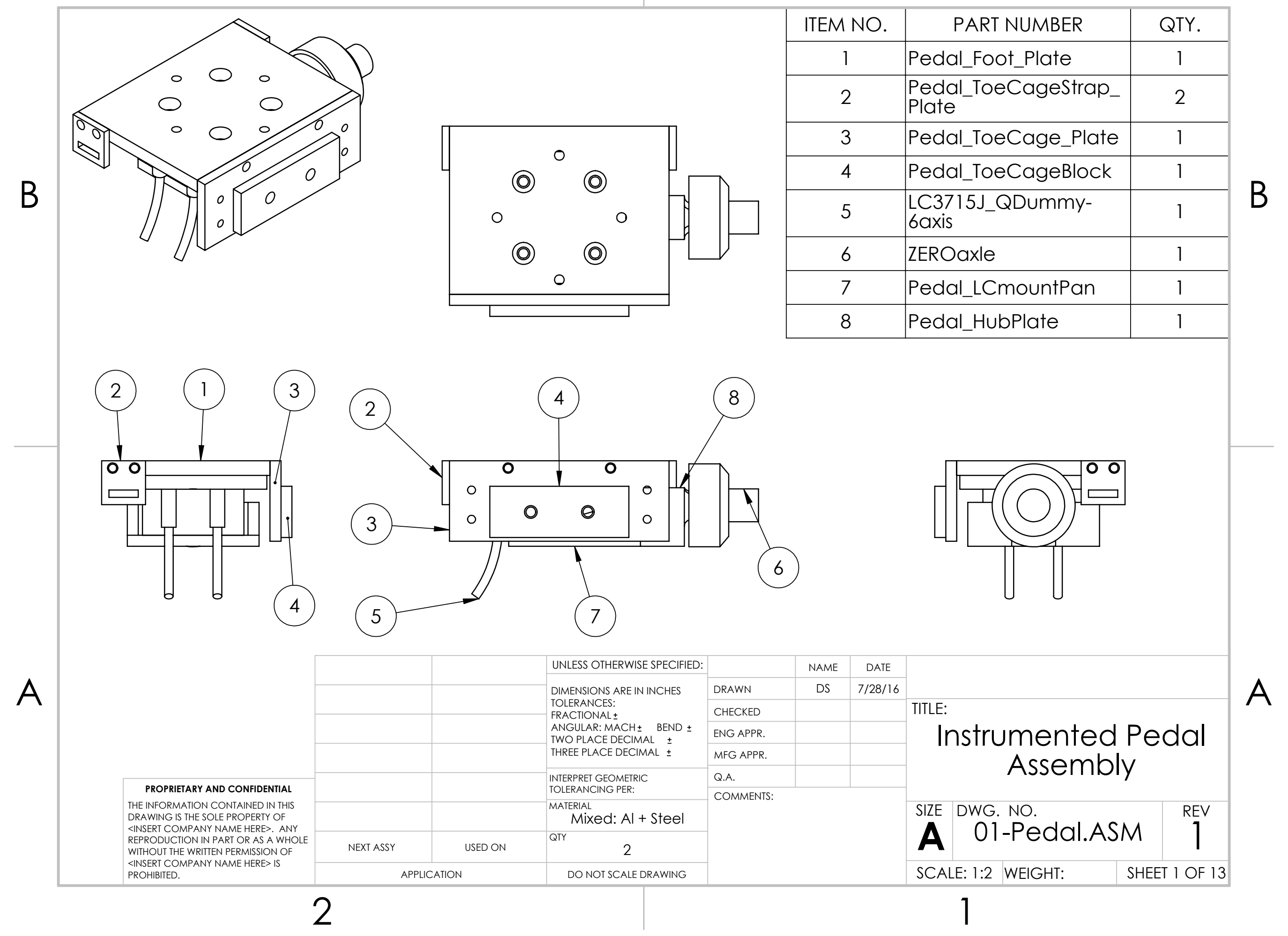




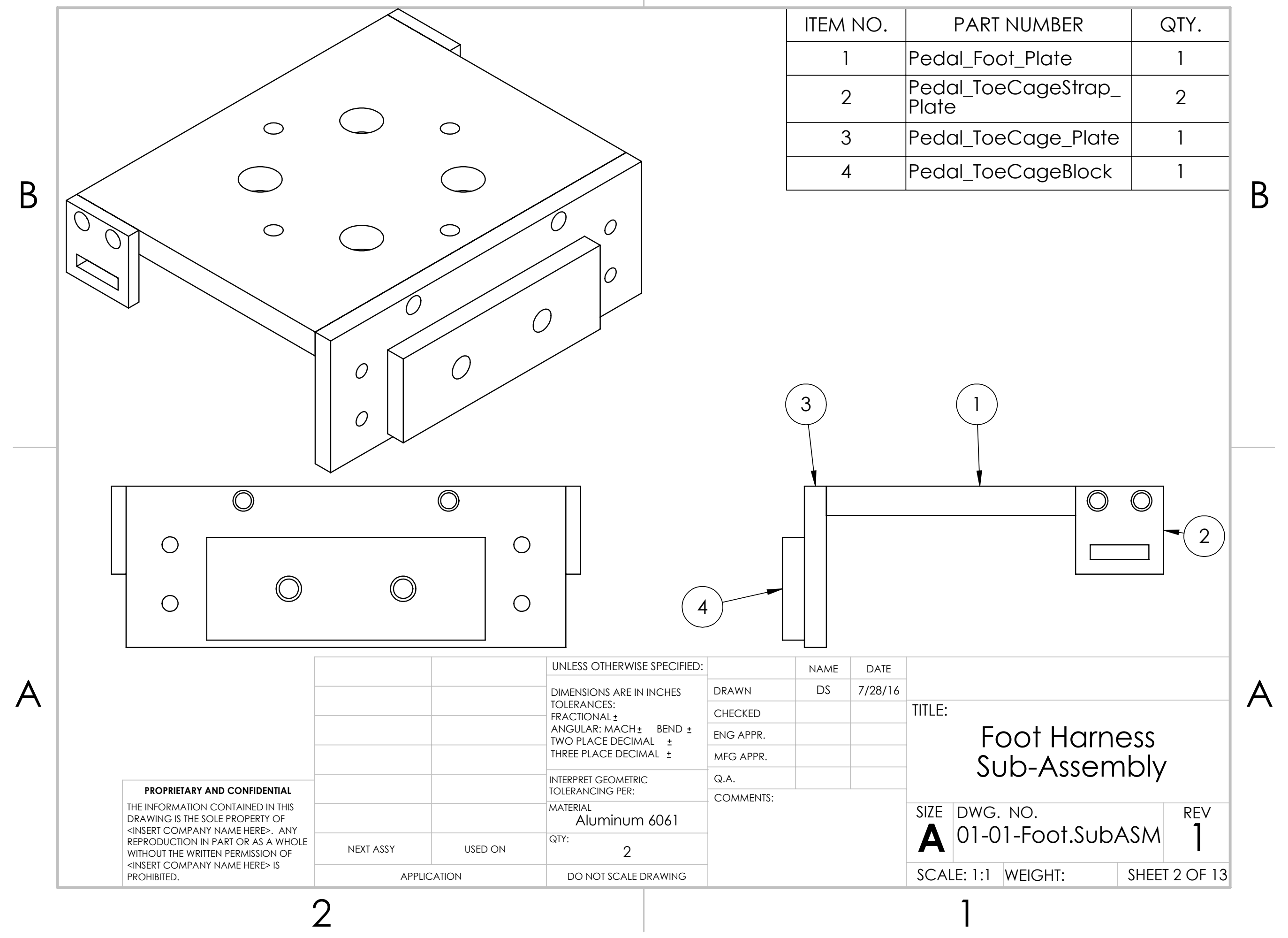


B

A
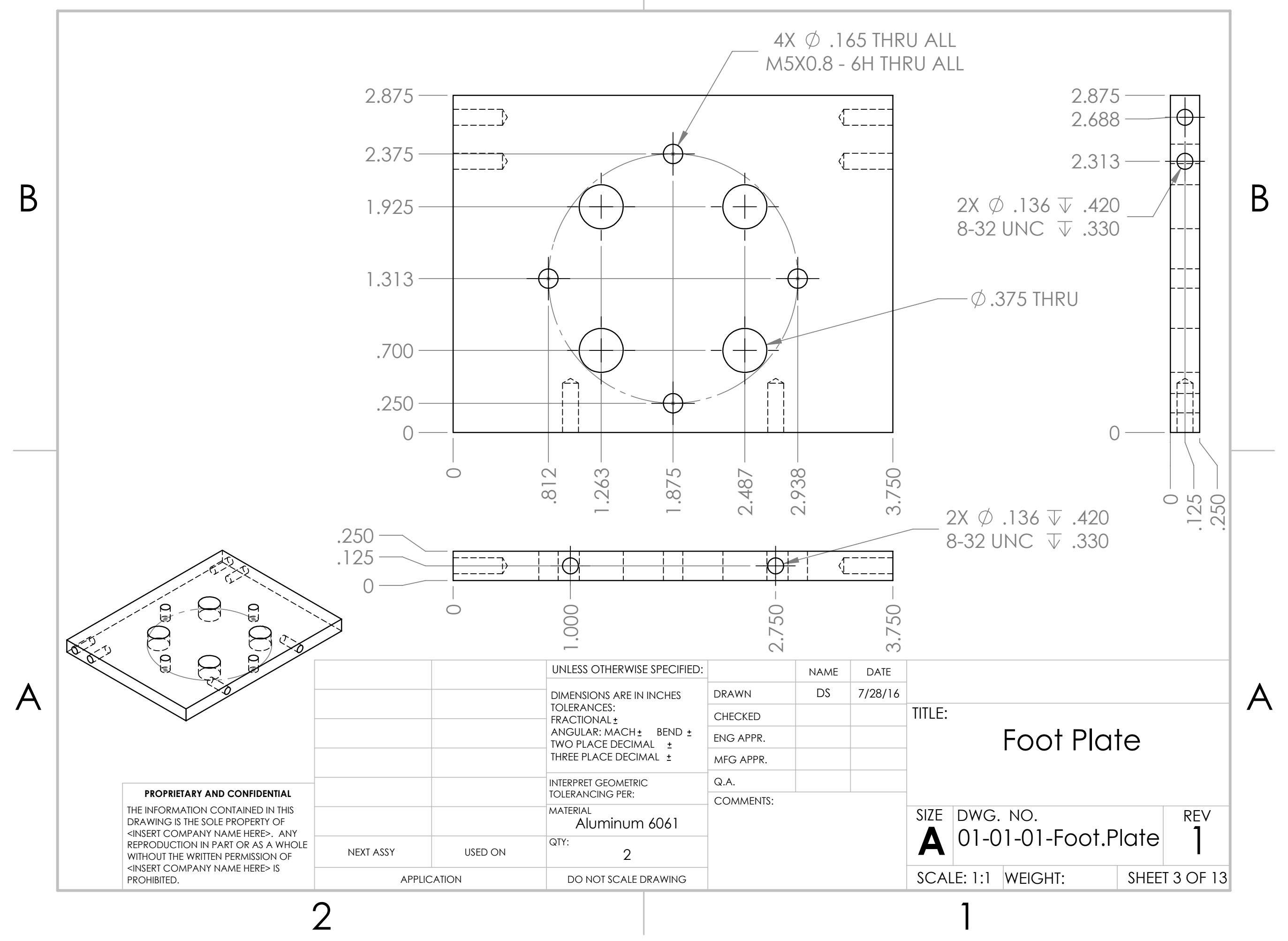


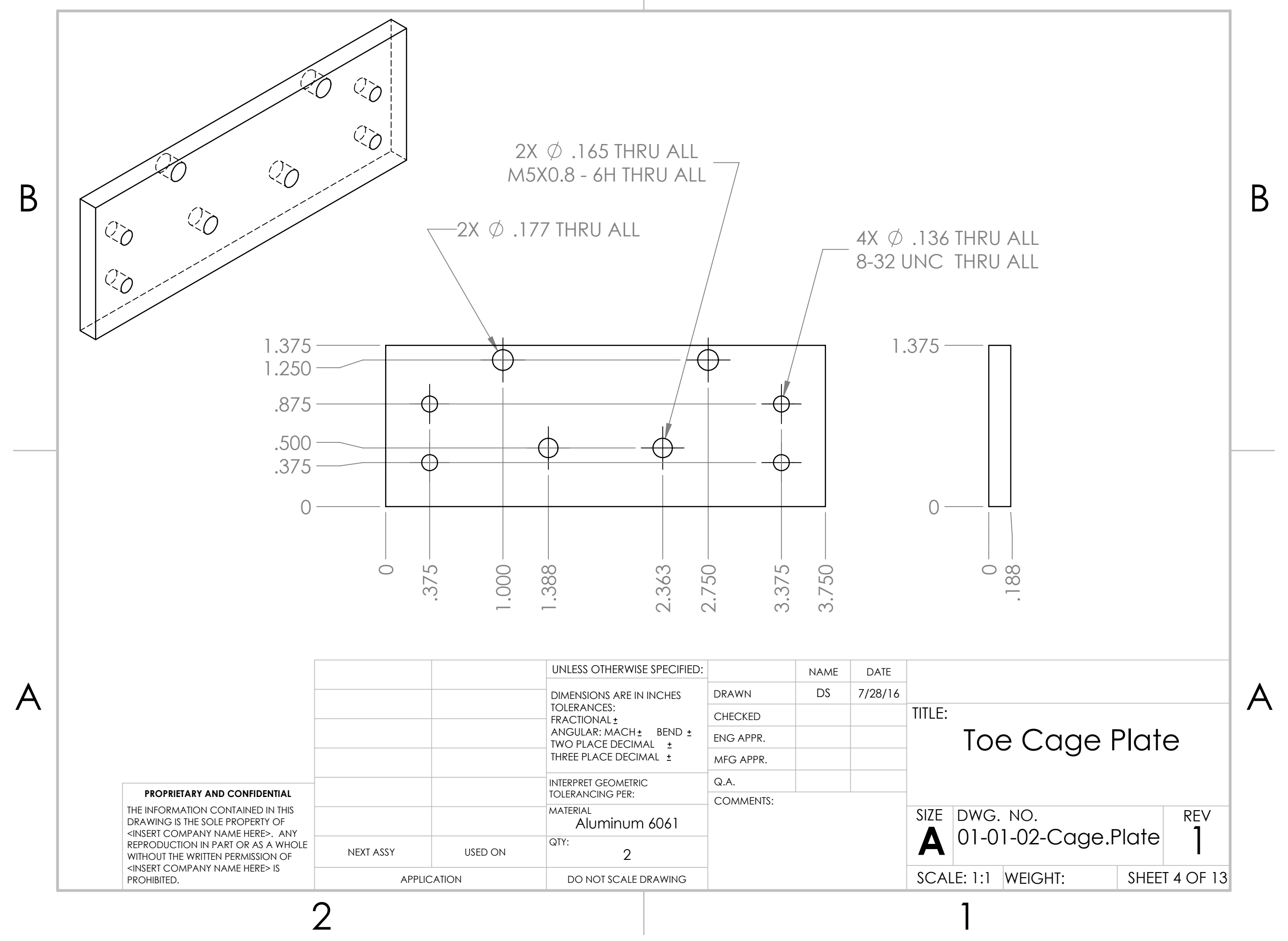


B

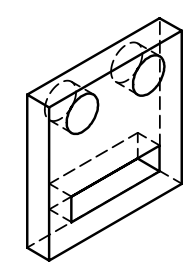

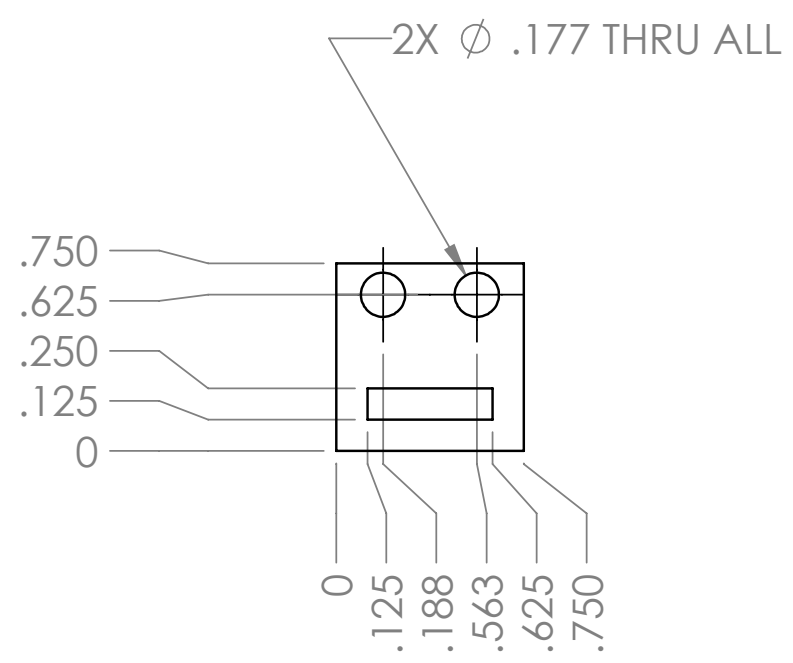

B

A

PROPRIETARY AND CONFIDENTIAL THE INFORMATION CONTAINED IN THIS DRAWING IS THE SOLE PROPERTY OF <INSERT COMPANY NAME HERE>. ANY WITHOUT THE WRITTEN PERMISSION OF <INSERT COMPANY NAME HERE> IS PROHIBITED.

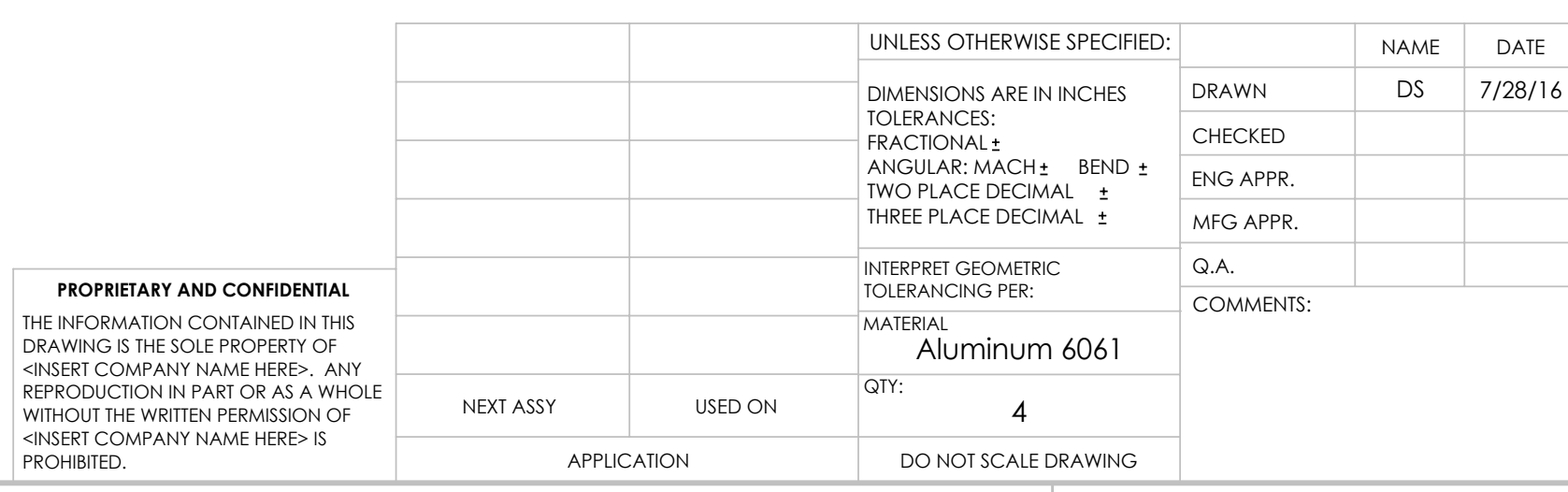

\section{TITLE:}

Toe Cage Strap Plate

SIZE DWG. NO.

A 01-01-03-Strap.Plate

SCALE: 1:1 WEIGHT: 


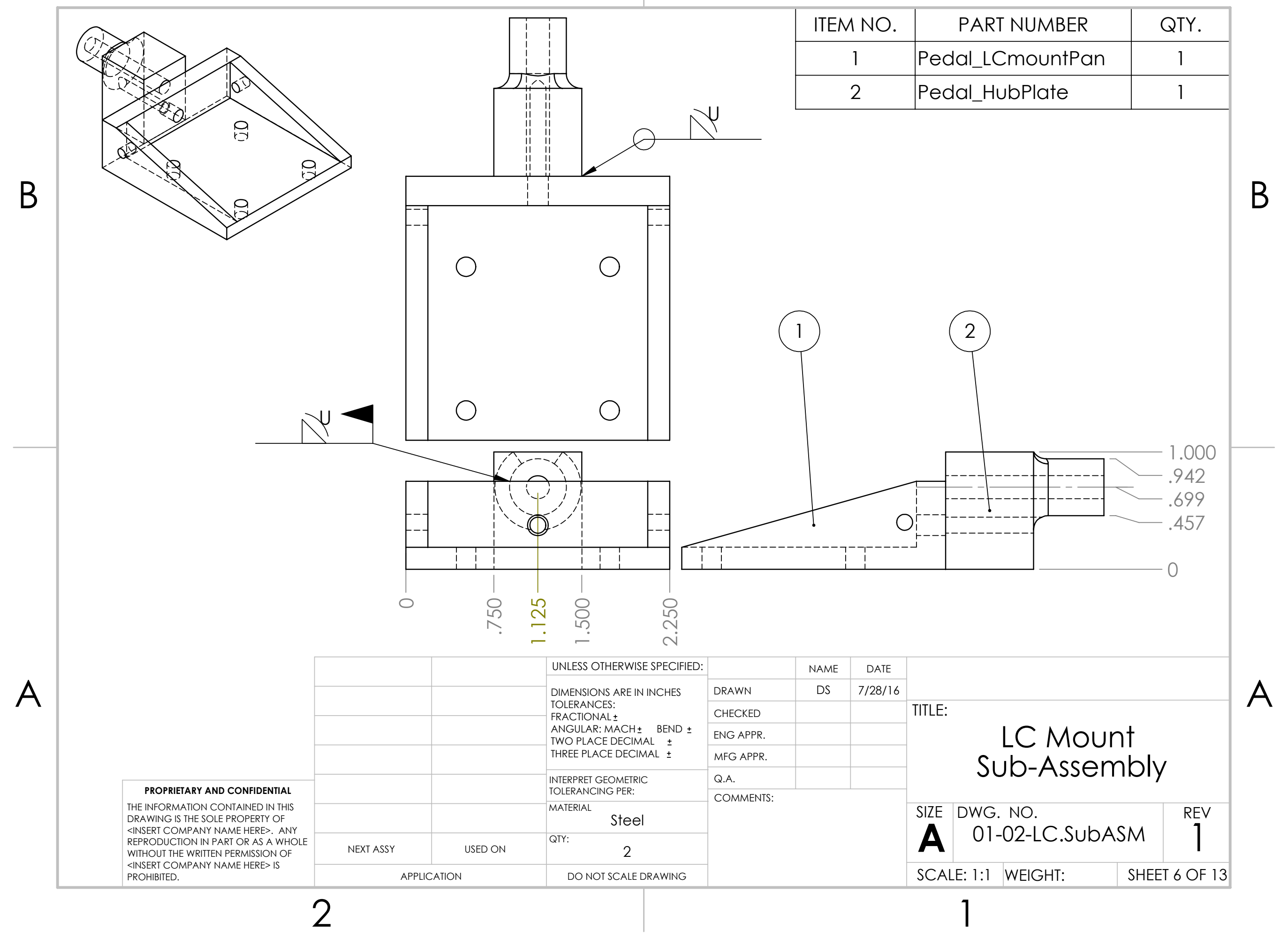


B

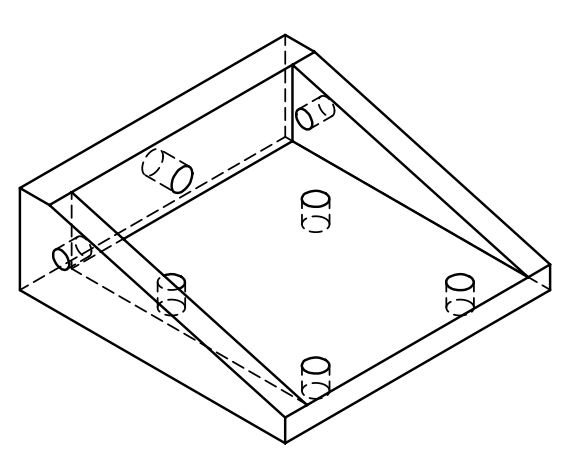

$\varnothing .136$ THRU ALL

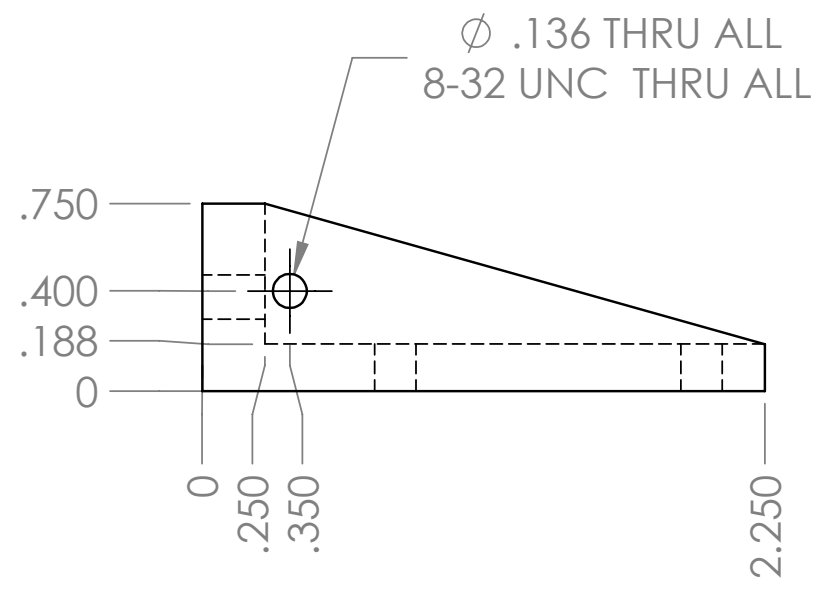

$4 X \varnothing .165$ THRU ALL M5X0.8 - 6H THRU ALL

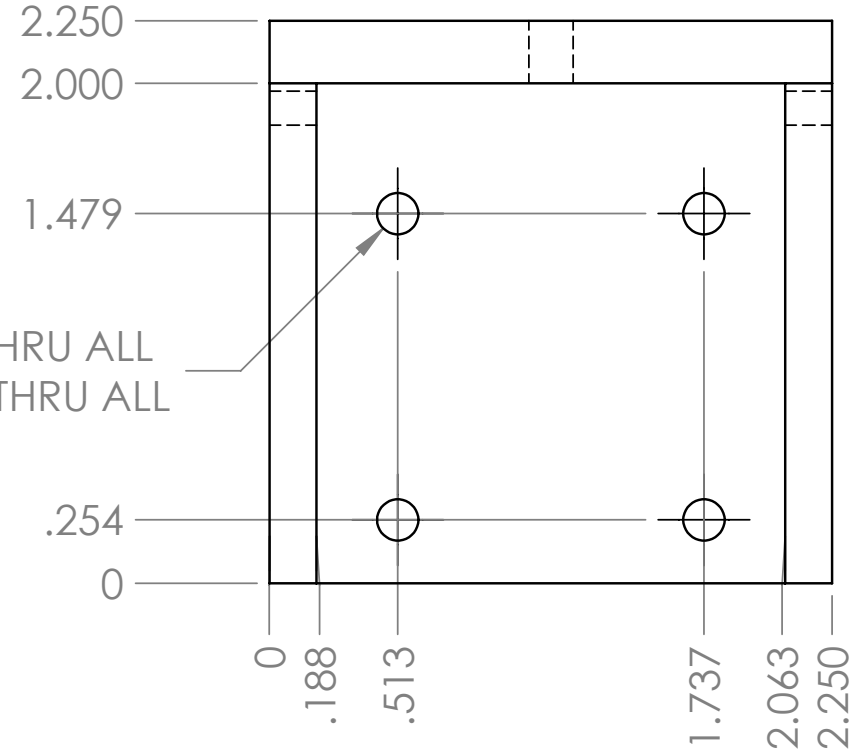

$\varnothing .177$ THRU ALL

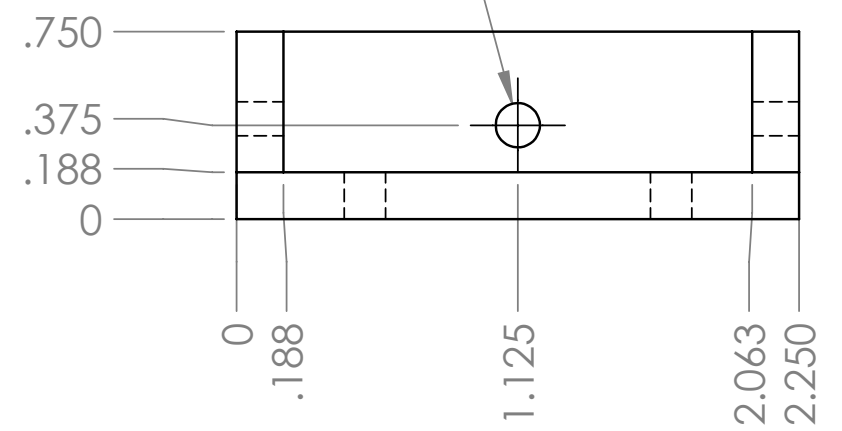

A

\begin{tabular}{|c|c|c|c|c|c|}
\hline & & UNLESS OTHERWISE SPECIFIED: & & NAME & DATE \\
\hline & & \multirow{4}{*}{$\begin{array}{l}\text { DIMENSIONS ARE IN INCHES } \\
\text { TOLERANCES: } \\
\text { FRACTIONAL } \pm \\
\text { ANGULAR: MACH } \pm \text { BEND } \pm \\
\text { TWO PLACE DECIMAL } \pm \\
\text { THREE PLACE DECIMAL } \pm\end{array}$} & DRAWN & DS & $7 / 28 / 16$ \\
\hline & & & CHECKED & & \\
\hline & & & ENG APPR. & & \\
\hline & & & MFG APPR. & & \\
\hline & & \multirow{2}{*}{$\begin{array}{l}\text { INTERPRET GEOMETRIC } \\
\text { TOLERANCING PER: }\end{array}$} & Q.A. & & \\
\hline & & & \multirow{4}{*}{\multicolumn{3}{|c|}{ COMMENTS: }} \\
\hline & & MATERIAL Steel & & & \\
\hline NEXT ASSY & USED ON & QTY: & & & \\
\hline \multicolumn{2}{|c|}{ APPLICATION } & DO NOT SCALE DRAWING & & & \\
\hline
\end{tabular}

TITLE:

\section{LC Mount Pan}

SIZE DWG. NO.

A 01-02-01-LC.Pan

REV

SCALE: 1:1 WEIGHT:
B

A 1 
B

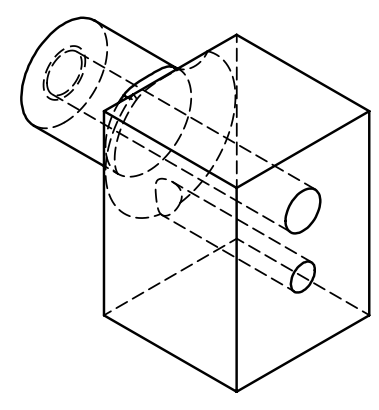

$\varnothing .136$ THRU ALL 8-32 UNC THRU ALL

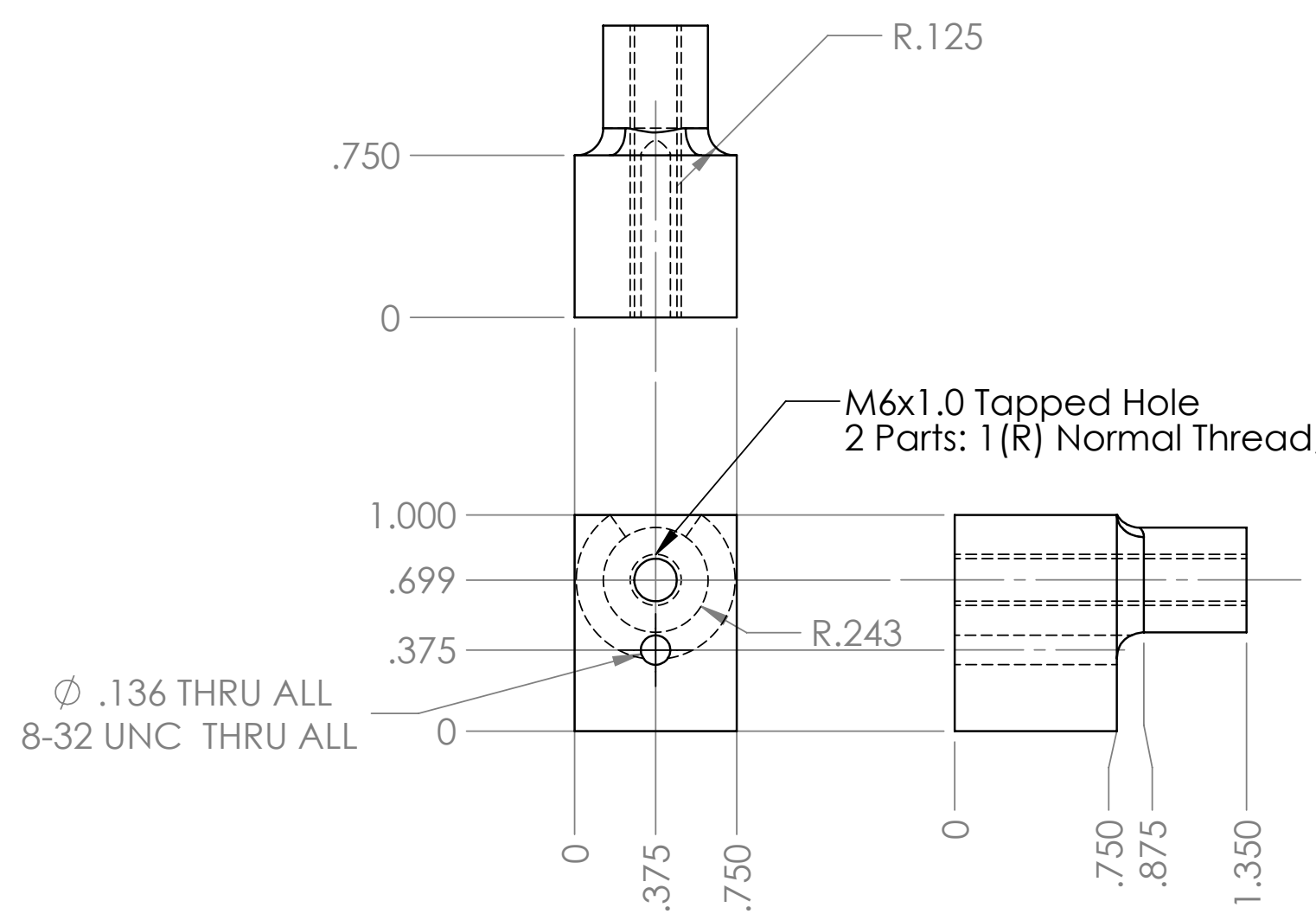

B

PROPRIETARY AND CONFIDENTIAL THE INFORMATION CONTAINED IN THIS DRAWING IS THE SOLE PROPERTY OF <INSERT COMPANY NAME HERE>. ANY WEPRODUCIOUN THE WRITTEN PERMISSION WO <INSERT COMPANY NAME HERE> IS PROHIBITED.

\begin{tabular}{|c|c|c|c|c|c|c|c|c|c|}
\hline & & & UNLESS OTHERWISE SPECIFIED: & & NAME & DATE & & & \\
\hline & & & \multirow{4}{*}{$\begin{array}{l}\text { DIMENSIONS ARE IN INCHES } \\
\text { TOLERANCES: } \\
\text { FRACTIONAL } \pm \\
\text { ANGULAR: MACH } \pm \text { BEND } \pm \\
\text { TWO PLACE DECIMAL } \pm \\
\text { THREE PLACE DECIMAL } \pm\end{array}$} & DRAWN & DS & $7 / 28 / 16$ & & & \\
\hline & & & & CHECKED & & & \multirow{5}{*}{\multicolumn{3}{|c|}{ TITLE: }} \\
\hline & & & & ENG APPR. & & & & & \\
\hline & & & & MFG APPR. & & & & & \\
\hline & & & \multirow{2}{*}{$\begin{array}{l}\text { INTERPRET GEOMETRIC } \\
\text { TOLERANCING PER: }\end{array}$} & Q.A. & & & & & \\
\hline \multirow{4}{*}{$\begin{array}{l}\text { PROPRIETARY AND CONFIDENTIAL } \\
\text { THE INFORMATION CONTAINED IN THIS } \\
\text { DRAWING IS THE SOLE PROPERTY OF } \\
\text { <INSERT COMPANY NAME HERE>. ANY } \\
\text { REPRODUCTON IN PAR OR AS A WHOLE } \\
\text { WITHOUT THE WRITTEN PERMISSION OF } \\
\text { <INSERT COMPANY NAME HERE> IS } \\
\text { PROHIBTED. }\end{array}$} & & & & \multirow{4}{*}{\multicolumn{3}{|c|}{ COMMENTS: }} & & & \\
\hline & & & MATERIAL Steel & & & & \multirow{2}{*}{\multicolumn{2}{|c|}{$\begin{array}{l}\text { DWG. NO. } \\
01-02-02-H u b . P l a t e\end{array}$}} & REV \\
\hline & NEXT ASSY & USED ON & QTY: & & & & & & \\
\hline & \multicolumn{2}{|c|}{ APPLICATION } & DO NOT SCALE DRAWING & & & & SCALE: 1:1 WEIGHT: & \multicolumn{2}{|c|}{ SHEET 8 OF 13} \\
\hline
\end{tabular}

A 


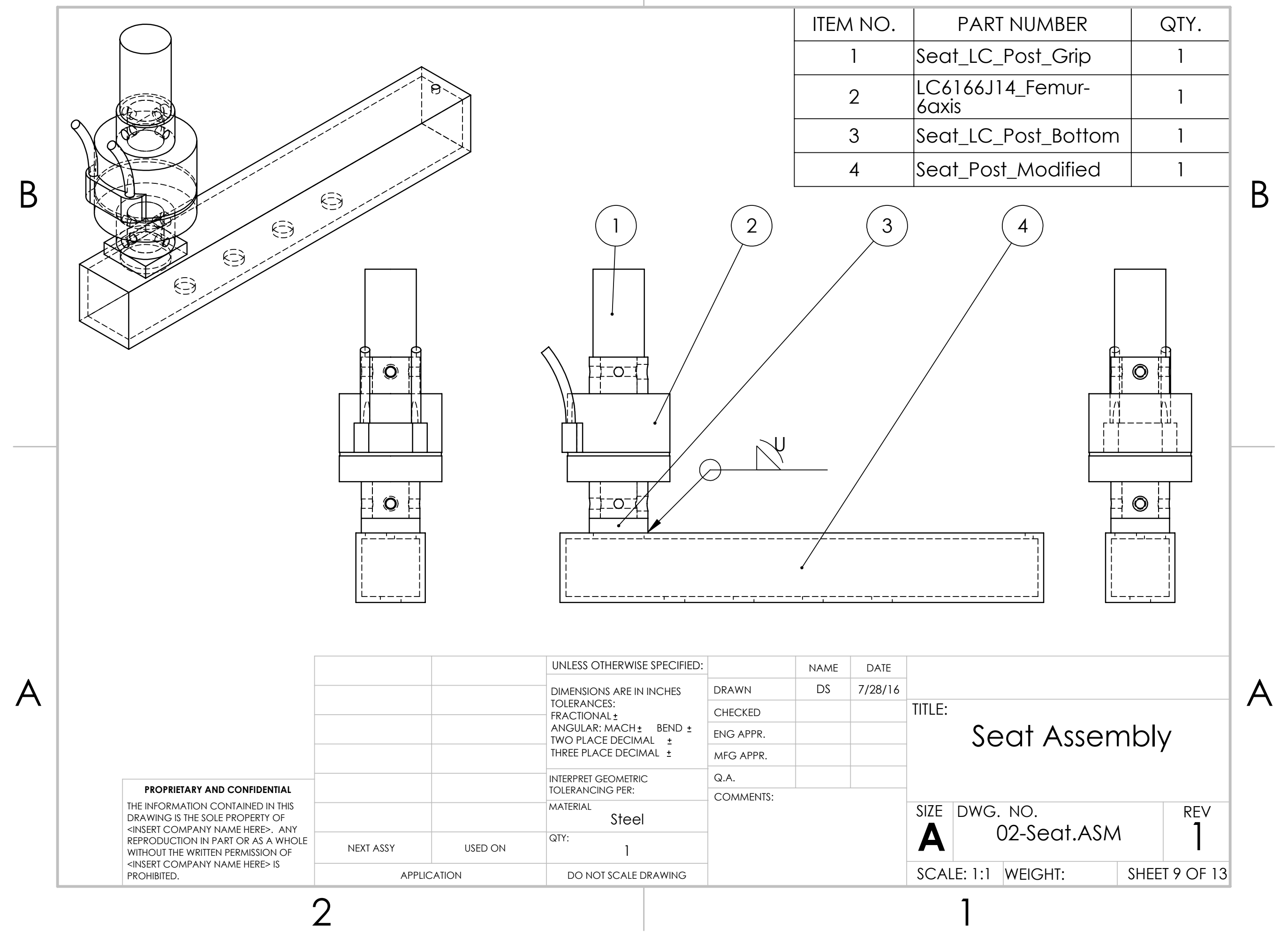




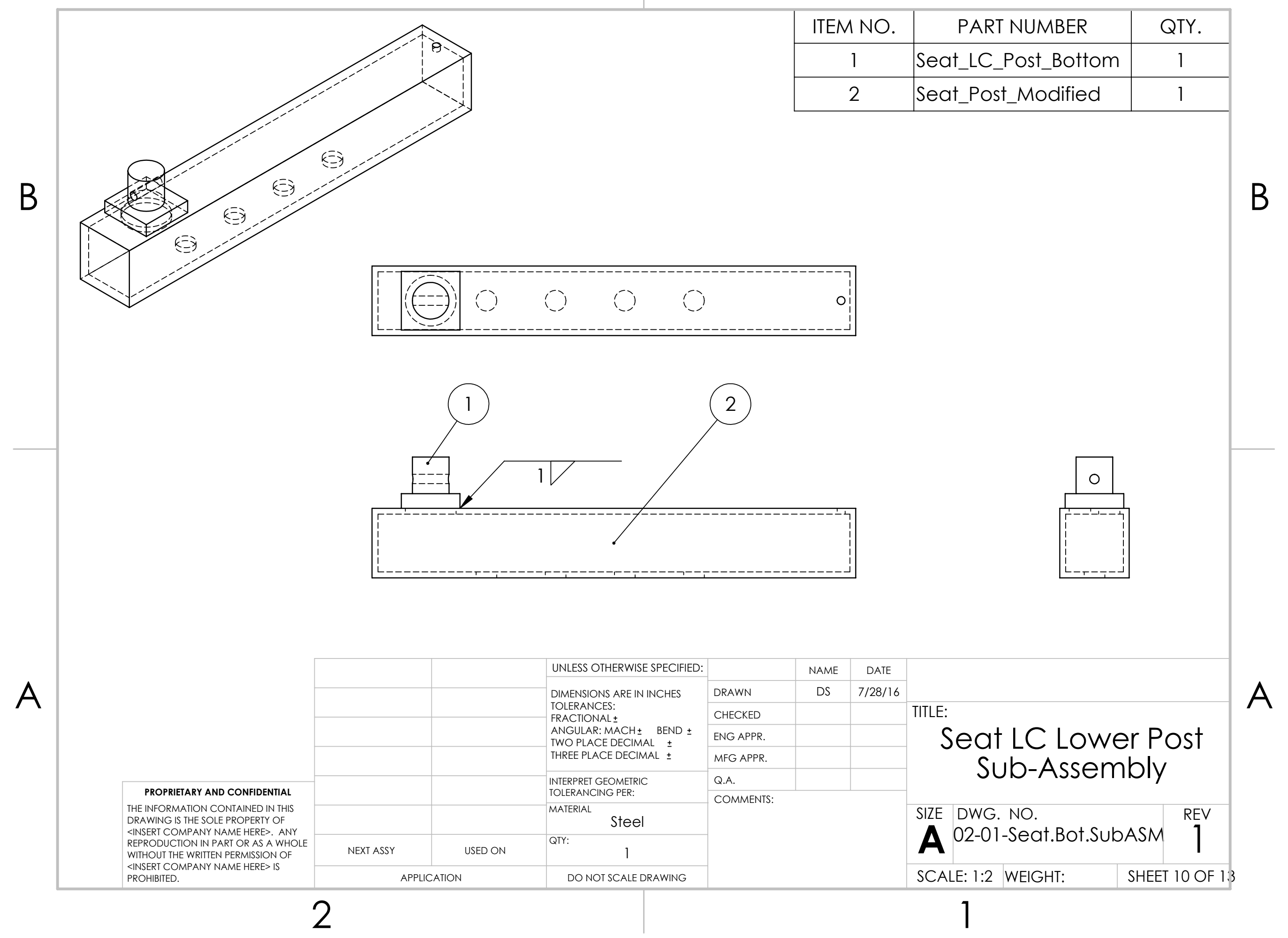




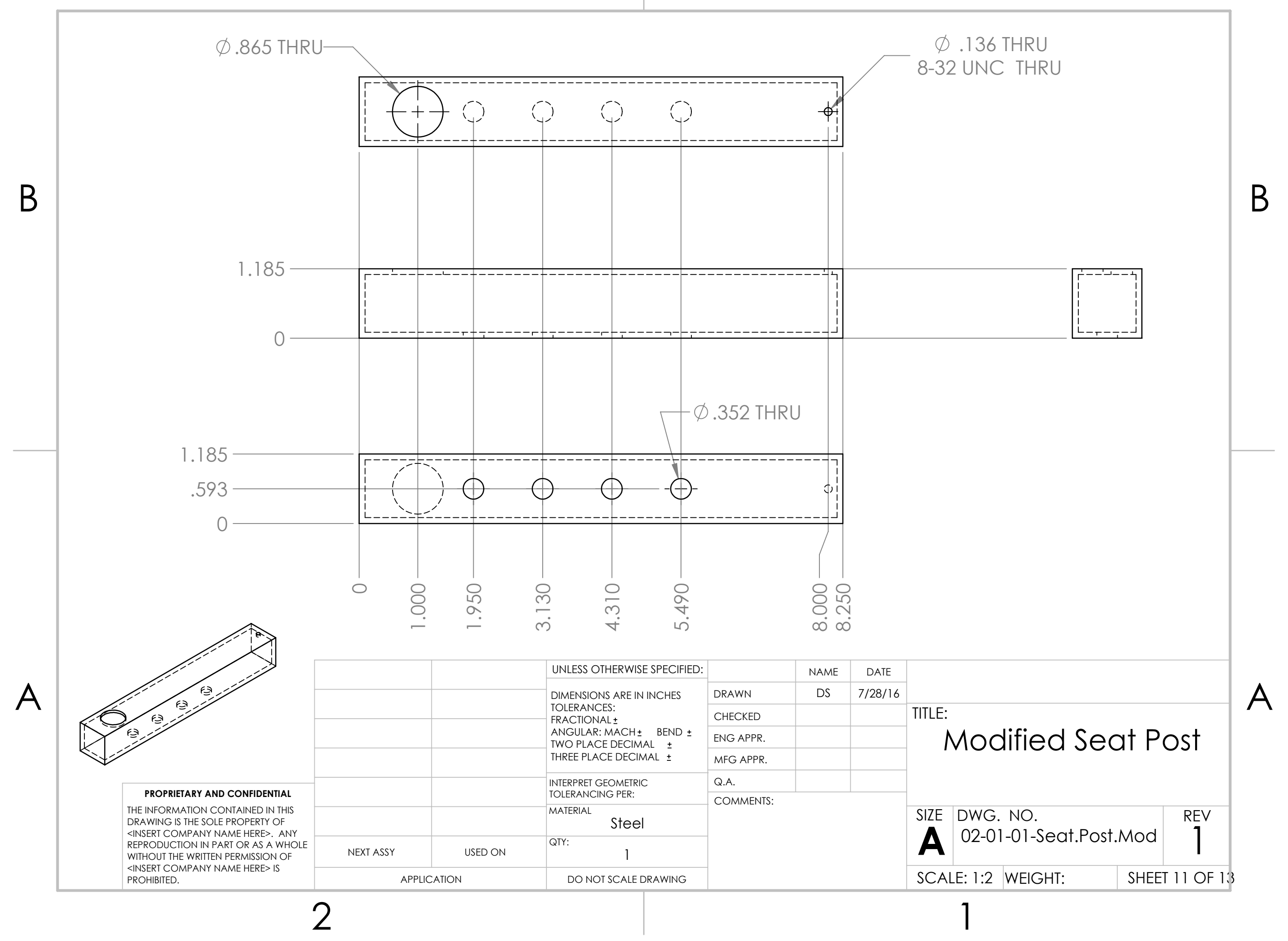


B

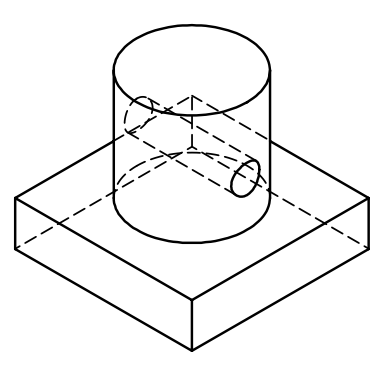

A

\section{PROPRIETARY AND CONFIDENTIAL} THE INFORMATION CONTAINED IN THIS DRAWING IS THE SOLE PROPERTY OF <INSERT COMPANY NAME HERE>. ANY WEPRODUCION NIN PART OR AS A WHO <INSERT COMPANY NAME HERE> IS PROHIBITED.

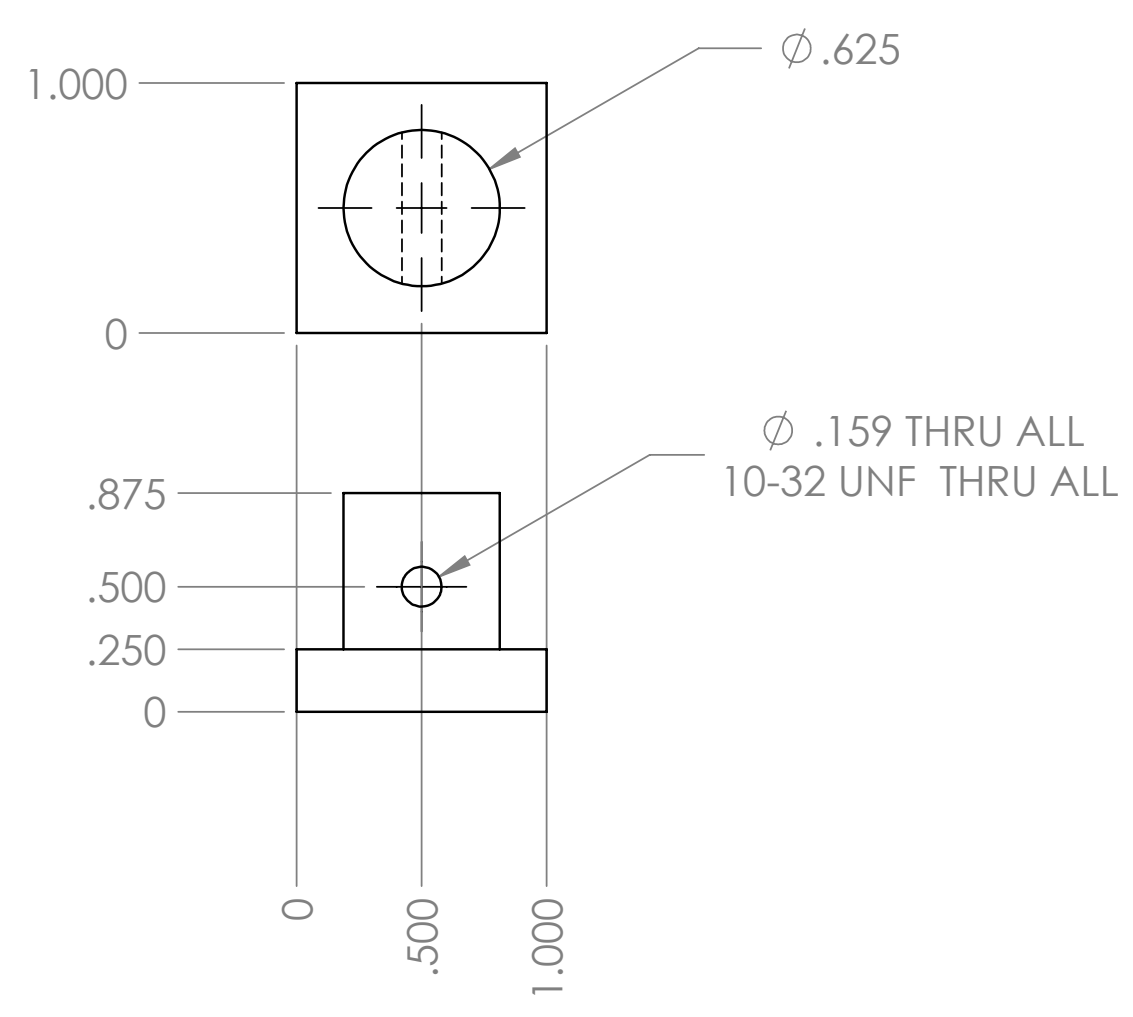

B

A Seat LC Bottom Post

\begin{tabular}{|c|c|c|c|c|c|}
\hline & & UNLESS OTHERWISE SPECIFIED: & & NAME & DATE \\
\hline & & \multirow{4}{*}{$\begin{array}{l}\text { DIMENSIONS ARE IN INCHES } \\
\text { TOLRANCES: } \\
\text { FRACTIONAL } \\
\text { ANGULAR: MACH } \pm \text { BEND } \pm \\
\text { TWO PLACE DECIMAL } \pm \\
\text { THREE PLACE DECIMAL } \pm\end{array}$} & DRAWN & DS & $7 / 28 / 16$ \\
\hline & & & CHECKED & & \\
\hline & & & ENG APPR. & & \\
\hline & & & MFG APPR. & & \\
\hline & & \multirow{2}{*}{$\begin{array}{l}\text { INTERPRET GEOMETRIC } \\
\text { TOLERANCING PER: }\end{array}$} & Q.A. & & \\
\hline & & & \multirow{3}{*}{\multicolumn{3}{|c|}{ COMMENTS: }} \\
\hline & & MATERIAL Steel & & & \\
\hline NEXT ASSY & USED ON & QTY: & & & \\
\hline \multicolumn{2}{|c|}{ APPLICATION } & DO NOT SCALE DRAWING & & & \\
\hline
\end{tabular}

SIZE DWG. NO.

A 02-01-02-Post.Bottom 1

SCALE: 1:1 WEIGHT:

SHEET 12 OF 1:3 

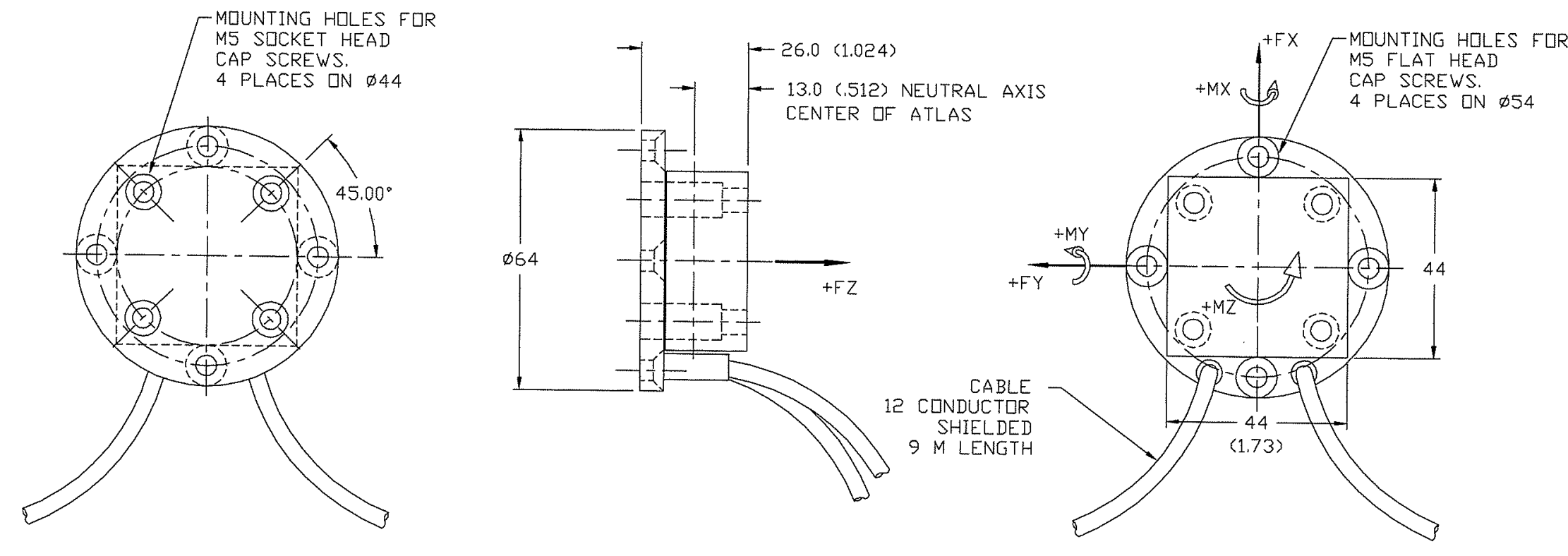
AXIS CAPACITY DUTPUT @ CAPACITY
$\begin{array}{lll}\text { FX } & 5.0 \mathrm{KN}(1125 \mathrm{LBF}) & 2.0 \mathrm{mV} / \mathrm{V} \\ \mathrm{FY} & 5.0 \mathrm{KN}(1125 \mathrm{LBF}) & 2.0 \mathrm{mV} \text { N }\end{array}$
$\begin{array}{llll}F Z & 5.0 \mathrm{KN}(1125 \mathrm{LBF}) & 2.0 \mathrm{mV} / \mathrm{V} \text { NDMINAL } \\ \mathrm{MX} & 6.0 \mathrm{KN}(1350 \mathrm{LBF} & \mathrm{mV} \text {. }\end{array}$

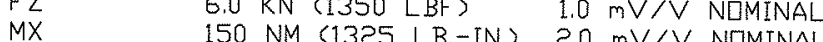
MY $\quad 150 \mathrm{NM}(1325 \mathrm{LB},-1 \mathrm{~N}, 2,0 \mathrm{mV} V \mathrm{~N}$ NUMINAL
$M Z \quad 80 \mathrm{NM}(700 \mathrm{LB}-I N)$

NUNLINEARITY $11 \%$ GF FULL SCALE

CRDSSTALK $<5 \%$ DF FULL SCALE

MODEL 3715 LIAD CELL CONFORMS TO J-211 PULARITY

FUR UPPER AND LOWER NECK PDSITIONS. TO CONFURM

TO J-211 PULARITY FUR LUMBAR PUSITIUN, THE

PULARITY GF FX AND MX MUST BE CHANGED.

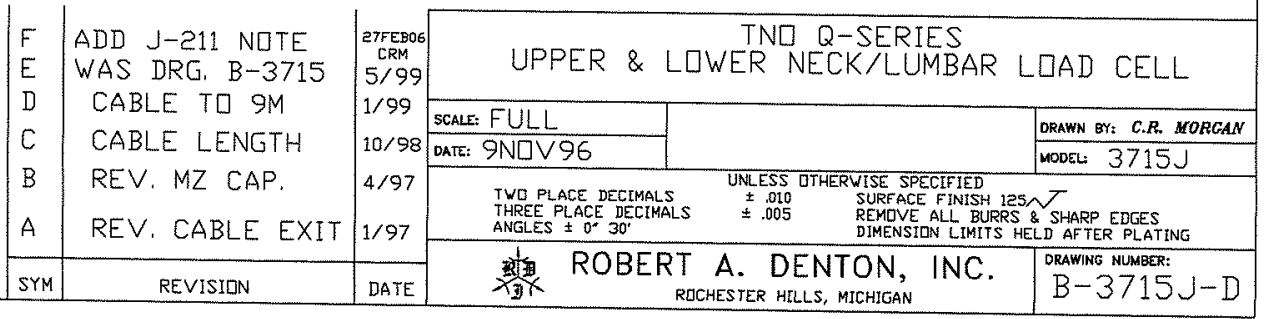


Preliminary Pedal Stress Concentration Analysis

800N Distributed Load On Foot Harness Plate

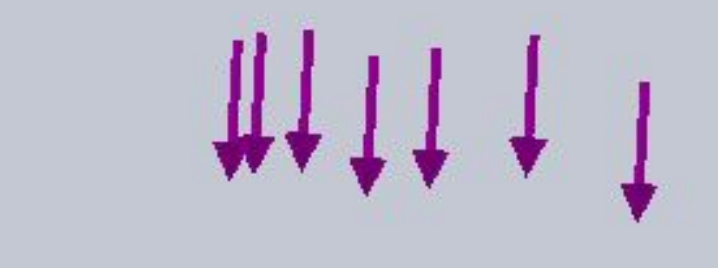

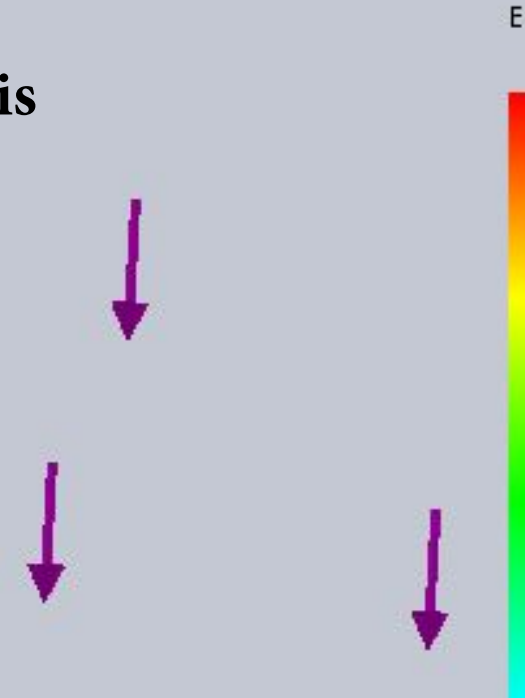

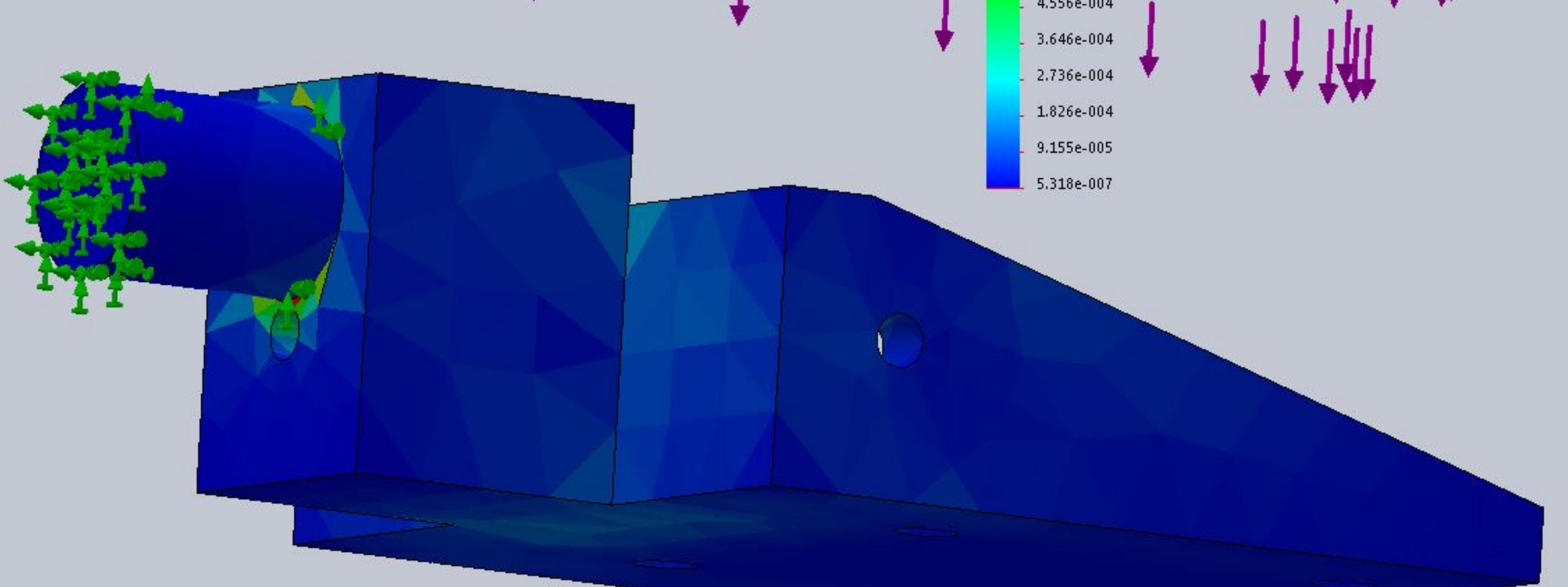

$1.093 \mathrm{e}-003$

$1.002 \mathrm{e}-003$

$9.107 \mathrm{e}-004$

$8.197 \mathrm{e}-004$

$7.287 \mathrm{e}-004$

$6.376 \mathrm{e}-004$

$5.466 \mathrm{e}-004$

$3.556 \mathrm{e}-004$

$2.736 \mathrm{e}-004$

$1.826 \mathrm{e}-004$

$\| \downarrow \downarrow \downarrow$

WW
$4.556 \mathrm{e}-004$ 


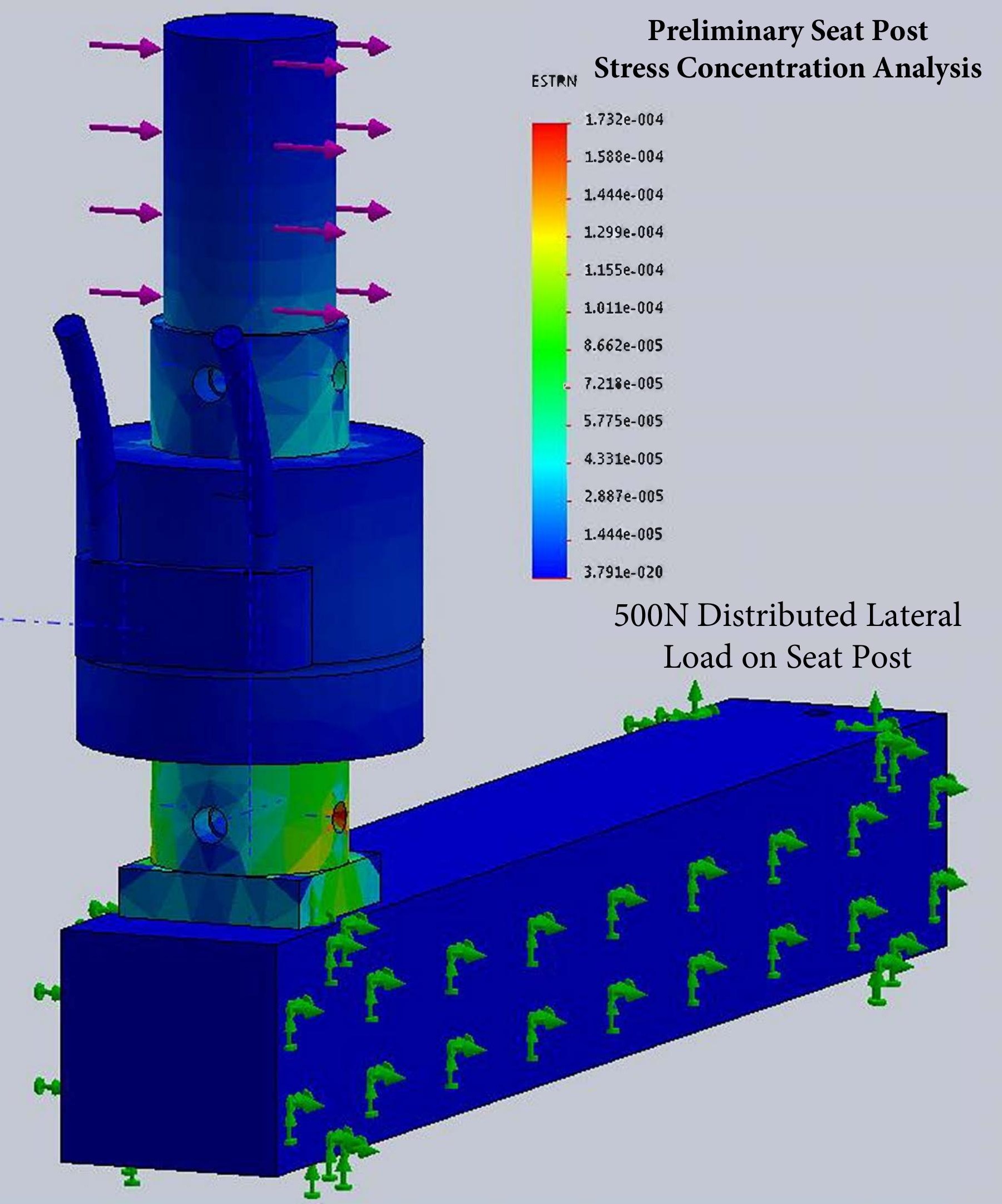

